\title{
Les premières occupations du Néolithique ancien dans le Nord-Ouest de la France
}

\author{
Ivan Praud*, Françoise Bostyn*, Nicolas Cayol**, Marie-France Dietsch-Sellamı***, \\ Caroline $\mathrm{HAMON}^{* * * *}$, Yves LANCHON ${ }^{\dagger}$, Nathalie VANDAMME*****
}

Reçu le 09/04/2018 - Accepté le 28/05/2018

\begin{abstract}
Mots clés. Néolithique ancien, Nord-Ouest de la France, chronologie, structuration des sites, activités économiques, tracéologie des outillages, décors céramiques, processus de néolithisation
\end{abstract}

Résumé. La découverte récente de plusieurs gisements datés du Néolithique ancien dans l'extrême Nord de la France s'est faite dans une région où cette période n'était pas attestée. Les dix sites présentés ici, sont tous datés de I'horizon chrono-culturel Blicquy/Villeneuve-Saint-Germain (BVSG) et sont implantés dans un secteur géographique compris entre les vallées de l'Oise à l'est, de la Somme au sud et des rivages de la Manche/Mer du Nord au nord-ouest. Cet effectif faible est aussi tardif pour un premier néolithique comparé aux occupations rubanées reconnues dans le Hainaut belge ou le Bassin parisien, plus importantes, mais aussi plus anciennes de quelques siècles. La synthèse des études a permis de préciser le cadre typo-chronologique de ces occupations à l'aide d'approches techno-fonctionnelles sur les différents mobiliers en les mettant en parallèle avec les mesures radiocarbone. Les comparaisons avec les sites du Néolithique ancien du Bassin parisien et de Belgique viendront alimenter la discussion sur les liens entretenus entre les différentes régions et permettront finalement de revenir sur les apports de ces sites dans l'étude plus générale des axes régionaux de colonisation néolithique rubanés et BVSG.
Kerwords. Early Neolithic, northwest of France, chronology, site structure, economic activities, usewear of tools, pottery decors, Neolithization process

Abstract. Recently, several sites dating from the Early Neolithic were discovered in the extreme North of France, in a region where this period had been unknown up until now. The ten sites presented here all date from the Blicquy/Villeneuve-SaintGermain (BVSG) chrono-cultural horizon, in a geographic sector located between the Oise valley to the east, the Somme valley to the south and the shores of the Channel/North Sea to the northwest. These sites are rather late for an Early Neolithic, compared to the more imposing Linear Pottery Culture settlements in Belgian Hainaut or the Paris basin, which are several centuries older. The synopsis of these studies defines the typo-chronological framework of these settlements, in parallel with the radiocarbon results and a techno-functional approach to the different objects. Comparisons with Early Neolithic sites from the Paris basin and Belgium contribute to the discussion on the links between the different regions and the broader question of regional axes of Linear Pottery Culture and BVSG.

\footnotetext{
* CRA Inrap, 11 rue des Champs, 59650 Villeneuve d'Ascq, Inrap Hauts-de-France, UMR 8215 Trajectoires | ivan.praud@inrap.fr | francoise.bostyn@inrap.fr

** CRA Inrap, Parc d'activités Noyon-Passel, 60400 Passel, Inrap Hauts-de-France, UMR 8215 Trajectoires | nicolas.cayol@inrap.fr

*** CRA Inrap, Domaine de Campagne, 24260 Campagne, UMR 5554 ISEM équipe Dynamique de la biodiversité, anthropo-écologie | marie-france.dietsch-sellami@inrap.fr

**** CNRS UMR 8215 Trajectoires, Maison de l'archéologie, 21 allée de l’Université, 92023 Nanterre cedex | caroline.hamon@mae.cnrs.fr

***** 10 rue de la Longue Paume, 02380 Coucy le Château Auffrique | vandamme.nathalie@yahoo.fr
} 


\section{The first Early Neolithic settlements in northwest France}

Over the past few years, several Early Neolithic sites have been discovered in the extreme North of France, in a region situated between the Oise valley to the east, the shores of the Channel/North Sea to the northwest and the Somme valley to the south (fig. 1). For a long time, the absence of Early Neolithic sites in this intermediate zone between the abundant Linear Pottery Culture sites and the Villeneuve-Saint-Germain in the Paris basin valleys (Aisne, Oise and Seine), and the Linear Pottery Culture settlements and the Blicquy group in Belgian Hainaut was puzzling, as both sectors have many points in common in terms of the typological evolution of pottery during the second phase of Neolithic expansion -the Blicquy/Villeneuve-Saint-Germain (Constantin 1985) - and in terms of the composition of the lithic series (Bostyn 1994). Moreover, many researchers have highlighted long-distance contacts and exchanges between these zones, based on the presence of bracelets in schist in the Paris basin sites, which appears to come from the Armorican Massif and the Ardennes (Fromont 2003, Praud et al. 2003), and the presence of blades in middle Bartonian Tertiary flint from the Paris basin at the Belgian sites (Bostyn 1994 and 2008, Denis 2014a).

The ten sites presented here all date from the Blicquy/ Villeneuve-Saint-Germain (BVSG) chrono-cultural horizon. They represent all the currently known sites in this region.

In this article, we present a synopsis of the studies of these sites in order to describe the typo-chronological framework of these settlements, as well as techno-functional studies of the different objects, in parallel with radiocarbon measurements. Comparisons with Early Neolithic sites from the Paris basin and Belgium contribute to the discussion on the links between the different regions and the broader question of regional axes of expansion of the Neolithic, the Linear Pottery Culture and the BVSG.

\section{STRUCTURATION OF THE SITES}

The recently acquired archaeological data are very disparate in terms of quantity and quality. Some sites deserve more detailed presentation than others, as they contained sufficient quantities of objects to characterize them, such as Loison-sous-Lens (Pas-de-Calais), Languevoisin-Quiquery (Somme) or Vitry-en-Artois (Pas-de-Calais). On account of their corpus of objects and their structuration, these are the best documented reference sites (table 1). Partial or former data from diagnostics or surface surveys are included here to enrich interpretations. Due to the silty sedimentary contexts of the sites, none of them contained bone remains.

The sites are spread over three geographic zones: the lower Somme valley (Boves and Étouvie), the upper Somme valley (Languevoisin-Quiquery, Sancourt and Vermand) and the Scarpe/Deûle/Scheldt sector (Vitry-en-Artois, Noyelles-sousLens, Loison-sous-Lens and Valenciennes).

The site of Languevoisin-Quiquery is situated on the Santerre plateau. The excavation focused on a set of seven Early Neolithic pits spread over a surface of $1,800 \mathrm{~m}^{2}$ (Baudry et al. 2013; fig. 3). Due to the absence of post holes, we cannot confidently reconstruct the domestic structuration of the site. The state of preservation of the pits is rather poor. The variety and quantity of discovered objects (pottery, lithics and sometimes ornaments), as well as the data from the different studies, point to a settlement zone. The waste is similar in many respects to the waste generally found in the lateral pits of Danube houses.

The site of Loison-sous-Lens (Praud et al. 2010), is located in the north-eastern part of the Pas-de-Calais department in the centre of the silty plain of Gohelle. The site is on the slope of a south-facing silty mound. Overall, the land forms a slight ledge and then a light slope towards the base of the Souchez valley. After clearing the surface over nearly two hectares (fig. 6, B), several series were attributed to the BVSG culture, on the basis of the objects found. They consist of eighteen structures, mainly waste pits (13), several post holes (4) and a grave. The organization of the digging of these pits along an east/west axis recalls the layout of the lateral pits generally associated with "Danube" houses.

The site of Vitry-en-Artois-en-Artois "Chemin-Brûlé" (Pas-de-Calais) was excavated over a surface of $6,600 \mathrm{~m}^{2}$ and yielded remains from a BVSG settlement (Cayol et al. 2015). The site is implanted on the slope of a chalky mound to the east of Arras. The settlement consists of three structures, including a pit with a "Y"-shaped profile which contained most of the finds, and two small very levelled pits, with few objects. The age of the pottery is correlated to the final stage of the BVSG, a stage which had not been recorded up until now.

The sites make up a rather mixed bunch and the absence of house plans does not facilitate interpretation. It is difficult to determine the type of settlement.

The site of Loison-sous-Lens could be a pioneering first village whereas Languevoisin-Quiquery and Vitry-en-Artois appear to be sites with temporary or specialized activities, located near a dwelling. 


\section{MATERIAL PRODUCTIONS}

\section{POTTERY}

The pottery assemblages identified at these sites can be attributed to the Blicquy-Villeneuve-Saint-Germain culture. The series contain 148 specimens for a total weight of $18.7 \mathrm{~kg}$, one third of which are decorated. They come from five sites, including the site of Loison-sous-Lens which yielded almost half of the specimens. Overall, the pottery is not very well conserved, the surfaces are eroded, and the less deeply incised and impressed pottery is often difficult to identify and interpret. Low firing temperatures also render this type of pottery particularly fragile.

This corpus presents several shared typo-technological characteristics: the use of added tempers (ground and calcined bone, limestone, sand), coil-mounted recipients, a majority of simple shapes $(83 \%)$, accompanied by pots with an "S"-shaped profile and several bottles (fig. 13). On the other hand, no comparison of the percentages of the different categories of decoration can be undertaken due to the small number of objects (table 4). In detail, the incised fishbone decoration and the comb-impressed decoration are mostly from the site of Loison-sous-Lens (fig. 14). Stamped decoration alone or associated with incisions is more widely represented at the sites of Loison-sous-Lens and Vitryen-Artois. The triangle theme is very frequent in this corpus (fig. 15). "V"-shaped impressions by pinching the clay are only attested at Loison-sous-Lens. Two sites yielded pots with "V"shaped impressions made with a smooth and not very thick cord, by pulling from the edge towards a gripping element or by placing it horizontally under the edge. Several specimens bear nipple shapes beneath the edge. Smooth cord plastic decorations and double knobs are traditionally considered as characteristic of a recent, or final phase of the VSG and Blicquy cultures (Constantin 1985, Lanchon 1984, 2008).

For the sites of Loison-sous-Lens and Vitry-en-Artois, typological (decorative techniques) and especially technological (bone temper) comparisons point to a regional Blicquy facies of the vast BVSG culture, whereas the sites of Vermand, Languevoisin-Quiquery and Étouvie are attributed to the VSG facies of the Paris basin.

The existence of "fishbone" incisions and modelled "V"shapes, and the absence of real cords points to the attribution of the site of Loison-sous-Lens to a middle phase of the BVSG culture, while the rest of the sites are attributed to the recent and final phases of this culture.

\section{THE LITHIC ASSEMBLAGES}

The lithic assemblages present elements of convergence and divergence. The series from the sites of Loison-sous-Lens and Languevoisin-Quiquery will form the basis of our discussion (fig. 18) and the others will be used as far as possible to complete our observations.

The fundamental elements of BVSG flint assemblages (Bostyn 1994, Allard and Bostyn 2006, Denis 2014a) include the dual production of flakes/blades in local raw materials, at all the sites studied, but flakes largely outnumber blades. Blade production is particularly widespread at Loison-sous-Lens, and all of our technical observations or comments on the operating chain are strictly comparable to descriptions of assemblages in the Paris basin (Bostyn 1994, Allard 1999, Augereau 2004, Denis 2008), showing technical and, by extension, cultural unity in the BVSG geographic zone. However, studies of the lithic assemblages from this culture show a gradual decrease in blade production throughout time, ranging from about $20 \%$ during the early phases to much lower percentages during recent phases (fig. 37). The site of Languevoisin-Quiquery is clearly lacking in laminar products, which is an original feature for the BVSG.

The other well-represented unifying factor in the studied series is the presence of Bartonian flint from the Paris basin (fig. 22). The circulation of this raw material throughout the BVSG culture, apart from in the westernmost margins (Bostyn and Denis 2016, Charraud 2013, Bostyn et al. forthcoming a), is now an established fact, but the way it was diffused within villages is still a matter of debate (Bostyn and Denis 2016). Some sites only received blade products, such as Noyelles, Boves, Vermand and Étouvie, whereas non-cortical flakes have been observed at the sites of Loison-sous-Lens and Languevoisin-Quiquery. Therefore, this raw material seems to have circulated in the form of preformed cores, like in Belgian Hainaut (Bostyn 2008, Denis 2014a, Bostyn and Denis 2016), where we also find these products, in spite of the distance. At Languevoisin-Quiquery, the hypothesis of the transport of preformed blade cores in Cretaceous and Bartonian flint implies that these skills were lacking on site and that the knapper moved, whereas similar productions are recorded at Loison-sous-Lens where local knappers present high levels of skill and also worked Bartonian flint cores. This is an important element as the Bartonian blade products from Loison-sous-Lens differ from those from LanguevoisinQuiquery by their smaller dimensions. The average blade width from the former site is $19.3 \mathrm{~mm}$, which is comparable to those in Cretaceous flint (18.4 mm), as opposed to $21.9 \mathrm{~mm}$ for Languevoisin-Quiquery.

The minor role of end scrapers on flakes, which are less abundant than denticulates and retouched flakes, raises questions (fig. 26). Indeed, BVSG series are characterized by the predominance of end scrapers among flake tools (Bostyn 1994, Allard and Bostyn 2006). It is difficult to explain this deficit, but it may point to less hide-working and thus to different spheres of activity to those identified at other Paris basin sites. On the other hand, the preponderance of burins among blade tools is more traditional and this tool can represent more than half the blade tools. Plant working is well represented and the significant number of burins on flakes, in addition to burins on blades, tends to accentuate this sphere of activity (fig. 36). The presence of numerous sickle blade elements, on flakes as well as blades, attests to intensive agricultural activities.

Tranchets are part of the flake tool series at three sites (Loison-sous-Lens, Vitry-en-Artois and Boves), but are absent at Languevoisin-Quiquery. This tool appears at a rather late stage in the Paris basin, during the final phase of the BVSG, whereas it is present at three Hainaut sites, including 
Irchonwelz "la Bonne Fortune", which is attributed to the early phase of the BVSG (Constantin et al. 1978, Denis 2014a), Aubechies and Ellignies-Sainte-Anne.

If we accept that the Belgian Hainaut and the Paris basin phases are synchronous, then we could consider that the tranchet appears during the early phase in Belgian Hainaut and is assimilated into the Paris basin tool panoply at a later stage. Nonetheless, the absence of this type of tool at LanguevoisinQuiquery is puzzling as this occupation dates from the end of the BVSG. This could, once again, point to the original status of this site, linked to site function. This would explain the presence of second choice (cortical and crested) blade products.

The arrowheads are almost exclusively from the site of Loison-sous-Lens (with one from Vermand; fig. 35). The traditional perforating type, with inverse retouch on the base, is associated with a comparable quantity of cutting arrowheads. The latter also appear in large numbers during the final phase of the BVSG, at the sites of Hainaut, but several specimens are mentioned at the site of Irchonwelz "la Bonne Fortune" (Farruggia et al. 1982) and Blicquy "la Couture du Couvent" (Constantin et al. 1991), during the early phase.

\section{MACRO-TOOLS}

The discovery of a particularly large number of grinding tools associated with manufacturing waste at Loison-sous-Lens (93 objects in sandstone for a weight of $165 \mathrm{~kg}$ ) is noteworthy. This contrasts sharply with the paucity of objects in sandstone in the valley of the Somme, at Sancourt (one trimming flake from a grinding tool) or downstream at Boves (three sandstone elements).

Altogether, the site yielded twelve querns, ten grinders, five fragments of grinding tools or manufacturing (four primary flakes, 41 shaping flakes) or maintenance flakes (6 trimming flakes; table 7). The presence of pairs of querns and grinders and the intact condition of practically all the twenty-two querns and grinders is exceptional for the BVSG. Four main themes will be addressed below: the typological diversity of the querns, tool function, site specialization in food preparation in comparison with "classical" settlement sites and lastly, the significance of the deposition or abandonment of large quantities of grinding tools at BVSG settlement sites.

Three different types of querns are represented: the thinnest on trapezoidal or quadrangular plaques, thick querns with plane or concave surfaces with or without a proximal edge and a narrow and thick type (fig. 39 to 41). All the grinders can be assimilated to the short category, with two main types: ovoid grinders with a plane or convex active surface and quadrangular grinders with abrupt edges and an active plane surface. The typology of the tools from Loison-sous-Lens is very similar to neighbouring regions. They are comparable to the tools from Hainaut, Irchonwelz or Blicquy (Constantin et al. 1978, Hamon 2008), and to those from the north of the Seine basin, in particular in the plain of France at Saint-Denis (Hamon and Samzun 2004), Trosly-Breuil, Bucy-le-Long le Fond du Petit Marais or la Fosse Tounise in the Aisne valley (Hamon 2006), Longueil-Sainte-Marie in the Oise valley (Bostyn et al. 2015), or Ocquerre (Hamon 2009) and Luzancy (Hamon 2013) in the Marne valley. The use of grinding systems with short grinders used for back and forth actions is thus standard practice at Loison-sous-Lens. However, the secondary use of circular grinding was brought to light for at least one quern, and probably two others. The use-wear analysis of five querns and three grinders showed that they were used for grinding cereals.

Several questions regarding the composition and nature of this assemblage are still pending. Why were no grinding tool remains or any other sandstone tools found in the pits, as is generally the case in the lateral pits of BVSG settlement sites?

Finally, the absence of any specific organization of these querns also seems to exclude the deposition of the querns in situ. They seem to be related to discharge areas of grinding tools, abandoned in almost the same position in which they were used, associated with some of the manufacture and maintenance waste. The crude shaping of some querns, the presence of atypical forms (quern no. 4 from pit 11) and the fresh and partial sharpening of some of the active surfaces (ST 11 and 1074) indicate a high demand for grinding tools at the site. An episode of the intensive use of querns for grinding cereals at the end of the BVSG settlement of the site could explain this specific configuration.

\section{ORNAMENTS}

The series is made up of 33 fragments, with a minimum number of individuals evaluated at 19. Most of the ornaments discovered are in schist materials and the rest are in fired clay.

Most of the schist cannot be accurately sourced but it may come from the Armorican Massif or the Ardennes, which are both in the BVSG sphere of influence.

However, the micaceous schist with cordierites, which was identified for the first time at the end of the 1990s (Praud et al. 2003), clearly comes from the thermo-metamorphic belts surrounding the granite massifs from the north and east of the Armorican Massif.

\section{CHRONOLOGY}

Three sites have been radiocarbon dated (table 11). The results show that most of the structures ( 8 out of 10 ) are spread over a chronological segment corresponding more or less to the accepted duration of the BVSG, which is 300 years (5000/4950-4700/4650 BC; Dubouloz 2003). The overlap between charcoal dates from Vitry-en-Artois, LanguevoisinQuiquery and charred hazelnut shell dates from the site of Loison-sous-Lens indicates that the samples and results are accurate (fig. 52).

The sites discovered in this geographic sector mostly belong to the recent-final phase of the BVSG, as far as can be judged from the analysis of the objects and the isotopic dates. The site of Loison-sous-Lens can be attributed to the middle phase of the BVSG and thus appears to be the oldest in this territorial expansion of agro-pastoral communities. 


\section{SITE STATUS AND EXCHANGE NETWORKS}

The techno-functional approach shows that the flint toolkit was mostly used for plant processing. The tools were used for maintaining natural areas and/or for hoeing crops (tranchet), for cutting and scraping plants (burin, denticulates), and in particular for harvesting cereals (sickle blades) and scutching plant fibres (only burins). Plant processing and transformation are largely preponderant, as confirmed by the presence of numerous burins and sickle elements on blades but also on flakes. Tranchets are lacking at the site of Languevoisin-Quiquery, whereas they are represented at three other sites (Loison-sous-Lens, Boves and Vitry-en-Artois). Overall, wood working is less represented and includes scraping, planing and cutting actions (denticulate, debris, retouched flake and end scraper adze).

The flint assemblage and the ornaments include non-local materials. The synthesis of the flint assemblages revealed the importance of Bartonian flint from the Paris basin in circulation networks in the northwest quarter of France and Belgium. It can be considered as an essential social and identity marker for the cohesion of this cultural group over a vast territory ranging from the Paris basin to Belgian Hainaut and from Lower Normandy to the upper part of the Seine valley (Bostyn 1994 and 2008, Bostyn and Denis 2016, Charraud 2013, Denis 2014a, Bostyn et al. forthcoming a). The tools made in Bartonian flint require considerable technical investment and are characteristic of this culture (burins, sickle blades, arrowheads), reinforcing this identity aspect (Bostyn 2008). This intense diffusion is thus once again confirmed here, in particular by the presence of blades in the Somme valley at Étouvie, far from the main circulation axes along the main valleys.

On the other hand, the situation is very different for the flint from Ghlin, which was only used at Vermand, confirming that the inhabitants of the Paris basin were not particularly attracted to these products, and emphasizing the role of the upper Oise valley as a milestone between these regions. The circulation of flint from Ghlin was thus mostly directed towards the Hesbaye sites (Denis 2014b). Considering the proximity of Loison-sousLens to the Belgian Hainaut sites, the absence of this flint there raises questions, but may be related to its involvement in the economy of the Hesbaye sites.

The main sites also yielded bracelets in stone or fired earth. Materials from the Ardennes Massif and the Armorican Massif are present at the sites of Loison-sous-Lens and LanguevoisinQuiquery, indicating transport over very long distances, way beyond the large valleys of the Paris basin (Praud et al. 2003), and as far as Belgium (Fromont 2003).

\section{CONTRIBUTION OF THE SITES TO UNDERSTANDING THE NEOLITHIZATION PROCESS IN NORTH-WESTERN FRANCE}

Other data renew our vision of the BVSG culture, such as the presence of wheat in slightly smaller quantities than emmer wheat in the carpological spectrum of Loison-sous-
Lens, whereas up until now, this naked wheat had only been identified in the recent BVSG in the Paris basin (DietschSellami 2007). The introduction of this new cereal occurred at the same time as another early technical innovation: the flint tranchet. This relationship is significant as it points to a change in agricultural activities (maintenance of cultivated fields and use of the tranchet for hoeing) for cultivating this naked wheat grain, which is more fragile than the hardy cereals. Up until now, wheat crops and tranchet manufacture were only known in the recent phase of the BVSG in the Paris basin. Thus, the chronological attribution of the site of Loison-sous-Lens to the middle phase, on the basis of pottery and radiocarbon dates, tends to age this association. In the current state of knowledge, the development of agricultural practices associated with a new flint toolkit occurs earlier in the Blicquy group zone of influence than in the Paris basin.

The geographic position of the sites raises questions about their affinities with the two main settlement basins: the South of Belgium and the Paris basin. As expected, on a regional scale, we observe affinities between the sites of the DeûleScarpe basin, on one hand, which appear to be linked to the south-western extremity of the Neolithic expansion of the Blicquy group, and on the other hand, between the sites of the Somme basin which appear to be under the influence of the Paris basin. The Blicquy group sites and sites in the North of France also display many elements in common, such as the choice to settle in a slightly hilly area, with a loess soil (plateau silts), near water resources.

Moreover, Loison-sous-Lens and the sites of the Dendre basin present many similarities, such as the widespread use of a ground bone temper in pottery, the fishbone decoration technique, the abundance of grinding tools and the early introduction of the tranchet. These elements point to the existence of a regional Blicquian tradition. At Languevoisin-Quiquery, the proximity of the Oise valley, similar pottery shapes and the use of a sandy temper from Ocquerre "La Rocluche" (Seineet-Marne; Praud et al. 2009b), as well as the high proportion of tools in Bartonian flint (half the tools), show that privileged links existed with sites in the north of the Paris basin.

\section{IMPLICATIONS FOR THE NEOLITHIZATION PROCESS: A DECLINE IN EXPANSION...}

According to our current knowledge, the geographic area under consideration here corresponds to a zone marked by the stopping of the earliest Neolithic expansion. In spite of the development of rescue archaeology, no Linear Pottery Culture sites have been discovered there up until now and only several pioneering sites dating from the BVSG spread to these new natural areas during the maximum expansion of the Early Neolithic in the northern half of France. For our region, this expansion occurred from the sites of Belgian Hainaut and the Paris basin.

The type of soils and the geological context cannot account for the paucity of Danube settlements in this sector. The substantial silty cover developed on a mainly chalky substratum, 
which forms a very conducive environmental context for the development of agricultural practices and the acquisition of lithic materials. Therefore, the absence of Neolithic populations before $4950 \mathrm{cal}$. BC is still enigmatic, whereas in Belgian Hainaut, the oldest sites date to 5400/5300 BC (Crombé et al. 2005). The natural context cannot explain this absence of Early Neolithic sites. This standstill or stabilization in Neolithic spatial diffusion could be linked to a high density of hunter-gatherer populations implanted on these territories and impervious to lifestyle changes. However, regional settlement during the final phase of the Mesolithic was rather inconsequential. The recent discovery and radiocarbon dating of a final Mesolithic site south of Lille (Féray et al. 2016) yielded an age of $6590 \pm 40$ BP (Beta-439839), or between 5616 and $5481 \mathrm{cal}$. BC (to 95.4\%). This time frame is earlier than the first extra-regional Linear Pottery Culture. For this terminal phase of the Mesolithic, the rare Somme valley sites do not provide any data on possible contacts or links between these populations (Ducrocq 2001). The only date is from the Mesolithic site of Castel $(6090 \pm 95$ BP, Gif-10419, or 5290-4788 cal. BC), which points to overlapping with the Early Neolithic but is too imprecise (five centuries).

Another explanatory factor could be linked to the physical appearance of the regional hydrographic network. It was difficult for rivers flowing through this region along a north/ south or east/west axis to carve out beds, as the valley bottoms were covered with thick loess deposits during the Upper Pleistocene. The flow rate was limited, slopes were slight, and very wide valleys led to the development of vast wet plains which were not always very practicable. In contrast, Linear Pottery Culture sites in the Seine basin are distributed along the main waterway routes. When the river network was particularly unsuited to long distance movements, isolated settlements could have formed, like the Linear Pottery Culture sites in Hainaut, which could not extend further south, whereas in the Paris basin, the valleys offered more navigable penetration routes along a southeast/northwest axis, and settlement extended until the Channel shores.

Neolithization on a regional scale is quite similar to the expansion observed in the north-western part of the Northern European plain. Our questions and unsolved problems regarding Neolithic extension are relatively similar to those encountered by our Belgian colleagues. Indeed, only the end of the $5^{\text {th }}$ and the beginning of the $4^{\text {th }}$ millennia $\mathrm{BC}$ correspond to the whole colonization of the territory, including the sandy regions further north which had not been settled by Neolithic populations until then (Crombé et al. 2005), more than a millennium after the first implantations on the silty terrains of southern Belgium. In the same way, the neolithization of the western part of the Netherlands observed around the Rhine and Meuse deltas shows irreversible changes in the lifestyles of hunter-gatherer populations with the emergence of Middle Neolithic 2 cultures (Louwe Kooijmans 2011) via slow acquisition processes of new technical and economic strategies. On the other side of the Channel/North Sea, in Great Britain, new Neolithic ways of life did not durably develop until the transition between the $5^{\text {th }}$ and $4^{\text {th }}$ millennia BC (Whittle et al. 2011).

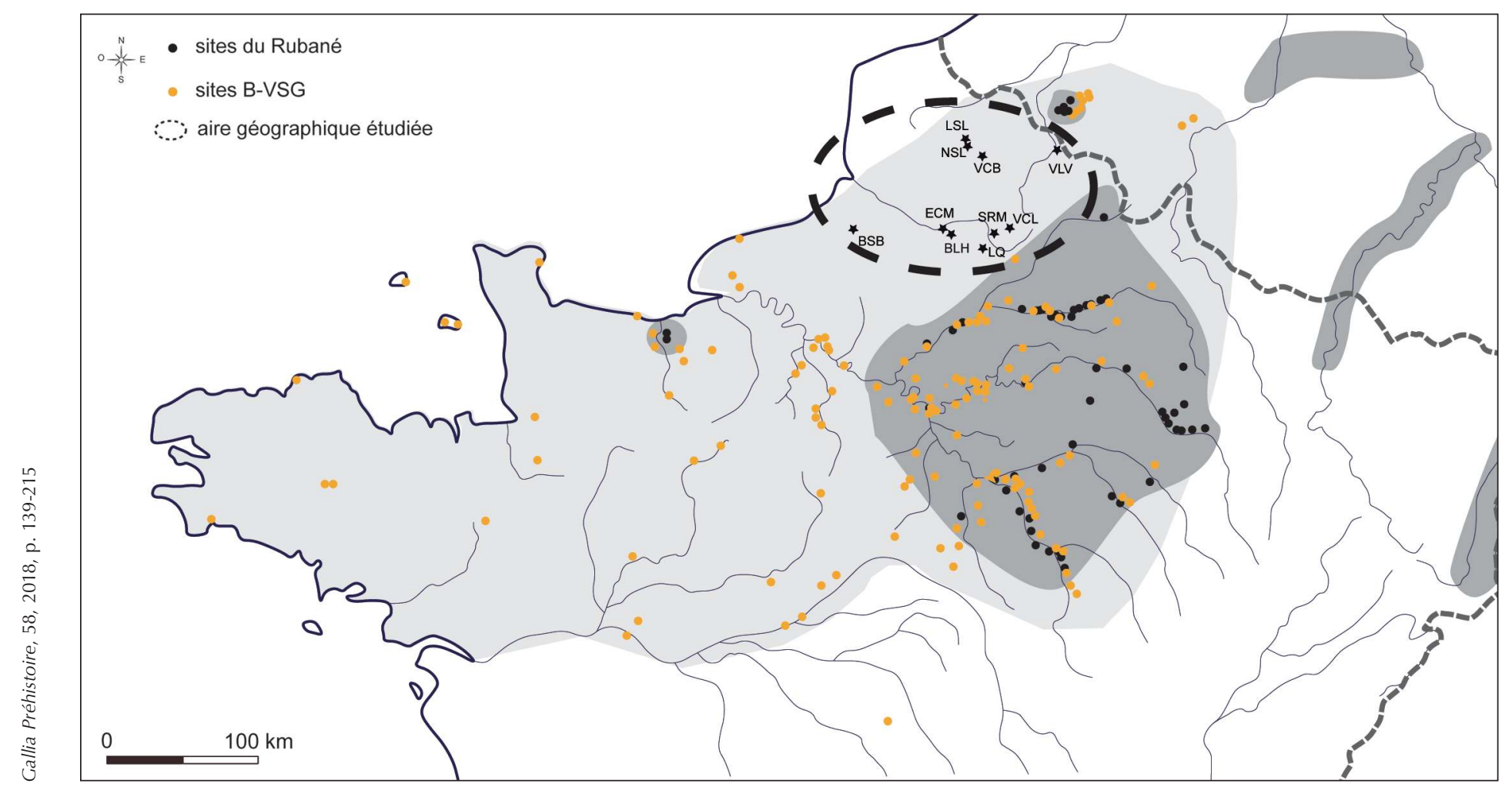


Ces dernières années, plusieurs sites datés du Néolithique ancien ont été découverts, dans le cadre exclusif d'opérations d'archéologie préventive, dans l'extrême Nord de la France, dans une région comprise entre la vallée de l'Oise à l'est, les rivages de la Manche/Mer du Nord au nord-ouest et la vallée de la Somme au sud (fig. 1), qui couvre plus ou moins parfaitement les départements actuels de la Somme, du Nord, du Pas-deCalais et le nord du département de l'Aisne.

Longtemps, l'absence de sites datés du Néolithique ancien dans cette zone intermédiaire entre les nombreuses occupations datées du Rubané et du Villeneuve-Saint-Germain des grandes vallées du Bassin parisien (Aisne, Oise et Seine), et les habitats du Rubané et du groupe de Blicquy dans le Hainaut belge, a constitué une véritable énigme alors que les points communs entre ces deux secteurs géographiques étaient nombreux tant dans l'évolution typologique de la céramique (Constantin 1985) que dans la composition des séries lithiques (Bostyn 1994). Les contacts et les échanges à longue distance ont, par ailleurs, été maintes fois soulignés par les chercheurs, notamment grâce à l'existence de bracelets en schiste sur les habitats du Bassin parisien, d'origine à la fois armoricaine et ardennaise supposée (Fromont 2003, Praud et al. 2003), et de lames en silex tertiaire du Bartonien moyen sur les gisements belges provenant des sites du Bassin parisien (Bostyn 1994 et 2008, Denis 2014a).

Ce vide avait été mis, sans doute un peu rapidement, sur le compte d'un manque conjoncturel de données que ne pourraient que remplir les fouilles préventives. Néanmoins, le développement des décapages surfaciques depuis plus de 30 ans dans toutes ces régions n'a pas apporté les informations espérées pour le Néolithique ancien alors que, dans le même laps de temps, le corpus de sites du Néolithique moyen ou du Néolithique final, par exemple, s'est considérablement étoffé (Monchablon et al. 2014, Joseph et al. 2011).

Fig. 1 - Carte d'extension maximale des aires culturelles du Rubané final du Bassin de la Seine (points noirs sur fond gris foncé, d'après Bostyn à paraître b) et du Blicquy-VilleneuveSaint-Germain (points orange sur fond gris clair) ainsi que les aires d'implantation du Rubané du Nord-Ouest en Belgique et en Alsace (DAO I. Praud, Inrap).

Map of the Linear Pottery Culture (LBK) in the Seine Basin (black dots on a dark-grey background, according to Bostyn à paraître b) and Blicquy-Villeneuve-Saint-Germain (BVSGorange dots on a light-grey background), as well as the areas where the Northwest LBK is located in Belgium and Alsace (CAD I. Praud, Inrap).

Liste des sites présentés dans cet article: List of sites presented in this article:

$\boldsymbol{B L H}$ : Boves « les Longues Haies (80), SRM: Sancourt "Grande rue, rue du Moulin » (80), ECM : Amiens "Étouvie» (80), LQ : Languevoisin-Quiquery (80), BSB : Blangy-surBresle (76), VCL : Vermand (02), VCB : Vitry-en-Artois (62), LSL : Loison-sous-Lens (62), NSL : Noyelles-sous-Lens (62), $V \boldsymbol{V} V$ : Valenciennes «Le Vignoble » (59).
Les dix sites présentés ici, sont tous datés de l'horizon chrono-culturel Blicquy/Villeneuve-Saint-Germain (BVSG). Ils représentent la totalité des sites connus à ce jour dans cette région. Au regard de la superficie concernée, cet effectif apparaît très faible, notamment par comparaison aux densités très importantes que l'on peut connaître pour le Rubané par exemple en Europe centrale (Pavùk 2004) ou, plus près de nous, dans la vallée de l'Aisne (Dubouloz et al. 2005), d'Alsace (Lefranc 2014) ou de la Hesbaye (Jadin 2003), mais aussi, pour le BVSG, dans les vallées du Bassin parisien. Les données présentées dans cette synthèse permettent ainsi de soulever plusieurs questions quant au processus de néolithisation de cet espace régional.

Les premières réflexions sur les processus de néolithisation de l'Europe sont déjà anciennes (Childe 1925), mais les dernières décennies ont été marquées par des discussions du modèle initial de colonisation linéaire proposé par G. Childe (ibid.), avec le développement de nouveaux modèles de diffusion par "vagues successives " (Ammerman et Cavalli-Sforza 1971) ou le modèle " arythmique » (Guilaine 2001) mettant en avant une progression irrégulière, parfois marquée par des arrêts qui peuvent durer plusieurs siècles. Plus récemment, ces discussions se sont enrichies de la prise en compte des facteurs climatiques et environnementaux comme facteurs importants dans les processus de néolithisation de l'Europe (Berger 2009). De manière encore plus globale, une approche de la colonisation rubanée en Europe tempérée a été construite à partir de la modélisation de plusieurs « agents » démographiques, environnementaux, économiques et sociaux permettant d'interroger, de comparer et d'expérimenter les différents scénarios de la diffusion dans l'espace et le temps des populations rubanées (Dubouloz et al. 2017). Les représentations cartographiques qui ont été proposées au fil du temps sont variées (Rasse 2008), mais elles ont en commun de montrer un espace vierge correspondant à notre zone d'étude, la posant d'emblée comme une région en marge des processus initiaux de la néolithisation du Nord de la France, largement véhiculée par la culture rubanée. Considérée comme postérieure à $5000 \mathrm{cal}$. BC, la culture de Blicquy-Villeneuve-SaintGermain n'a jamais été intégrée à ces discussions alors qu'elle représente, dans bon nombre de régions, la première culture néolithique. Dans une représentation cartographique plus récente de la diffusion du Néolithique en Europe, M. Rasse (2014) a étendu son approche au-delà de la fin du $6^{\mathrm{e}}$ millénaire, ce qui lui permet d'englober l'ensemble du territoire français. Néanmoins, si ces représentations permettent d'embrasser en un seul regard le phénomène à l'échelle de l'Europe, elles restent schématiques et masquent les disparités régionales.

Ainsi, on observe bien dans notre région d'étude, l'absence de sites attribués au Rubané. Ces derniers restent concentrés soit dans le Hainaut belge vers le nord-est, soit dans les vallées de l'Oise et l'Aisne vers le sud. Dans l'hypothèse, à ce jour non démentie, où il n'existe pas d'occupations plus anciennes du Rubané pour le Néolithique régional, se pose alors la question de l'origine et des axes de peuplement empruntés par les premiers cultivateurs pour s'implanter dans ces contrées. En effet, les sites étudiés ici se répartissent en 
deux groupes, le premier au nord, proche des sites hennuyers et le second centré sur la vallée de la Somme au sud et plus proche des grandes vallées de l'Oise et de l'Aisne. Ainsi, la colonisation néolithique a pu se faire soit à partir du Sud de la Belgique (Hainaut et Hesbaye) colonisé dès 5300 av. J.-C, soit à partir des régions colonisées dans le Bassin parisien autour de 5100 av. J.-C. Rappelons que pour les régions côtières nordoccidentales belges, il faut attendre plus d'un millénaire pour voir basculer les populations dans une économie agropastorale (Crombé et al. 2005), la nature sableuse des sous-sols semblant être une des causes majeures à cet arrêt de la progression des populations danubiennes. Cette colonisation a également pu se faire de façon différenciée à partir du Hainaut pour les sites du Nord, et via les habitats des vallées de l'Oise et de l'Aisne pour les sites les plus au sud de notre région d'étude, du fait d'une plus grande proximité géographique. En d'autres termes, plusieurs voies de colonisation néolithique peuvent être envisagées pour le Nord de la France.

La localisation des sites restant concentrée dans la partie orientale de notre zone d'étude, se pose la question des raisons d'un nombre si faible de sites et d'une répartition spatiale aussi contrainte. La densité des populations de chasseurs-cueilleurs autochtones était-elle telle qu'elle aurait pu freiner la progression des colons néolithiques? En d'autres termes, peut-on appréhender la question de la transition Mésolithique / Néolithique dans cette région, finalement assez comparable aux rivages plus septentrionaux de la mer du Nord (Whittle 2007) ? Dans quelle mesure les contextes pédologiques, proches de ceux du Hainaut, ont-ils ou non constitué un frein au développement du nouveau modèle économique ? Ou alors, comme cela a pu être mis en évidence dans le modèle arythmique, serions-nous ici dans une zone d'arrêt de la propagation du modèle économique rubané, avant nouvelle phase de colonisation à partir du BVSG ?

Autant de questions auxquelles nous nous proposons de réfléchir dans le cadre de cet article en synthétisant les études des sites qui sont, pour l'essentiel, restées à l'état de rapports, ou de présentations préliminaires dans des colloques. Il s'agira donc, tout d'abord, d'affiner le cadre typo-chronologique de ces occupations à l'aide des études techno-fonctionnelles sur les différents mobiliers en les mettant en parallèle avec les mesures radiocarbone. Les comparaisons avec les sites du Néolithique ancien du Bassin parisien et de Belgique viendront alors nourrir, dans un second temps, la discussion sur les liens entretenus entre les différentes régions pour finalement revenir sur les apports de ces sites à la discussion plus générale des axes de colonisation néolithique, rubanés et BVSG.

\section{STRUCTURATION DES GISEMENTS}

Les données archéologiques récemment accumulées sont en quantité et en qualité inégales. Plusieurs sites méritent une présentation un peu plus détaillée que les autres, car ils ont livré un mobilier suffisant pour en caractériser la nature comme Loison-sous-Lens (Pas-de-Calais), Languevoisin-Quiquery (Somme) ou Vitry-en-Artois (Pas-de-Calais). Ils constituent de fait par leur corpus et leur structuration, les gisements de référence les mieux documentés (tabl. 1). Les données partielles ou anciennes issues de diagnostics ou de ramassages de surface sont intégrées pour étoffer les interprétations. Enfin, un gisement occupe une position particulière car il n'a livré ni artefact ni creusement mais une séquence stratigraphique et pollinique couvrant l'Atlantique, référentiel qui nous permettra d'avoir des indicateurs sur les variations d'ordre climatique et anthropique. Rappelons également que, compte tenu des contextes sédimentaires limoneux, aucun de ces gisements n'a livré de restes osseux nous privant d'informations d'importance sur les relations entre sociétés humaines et animales - sauvages ou domestiques (Bedaut et Hachem 2008).

Les ensembles se répartissent en trois groupes géographiques : la vallée de la Somme aval (Boves et Étouvie), la vallée de la Somme amont (Languevoisin-Quiquery, Sancourt et Vermand) et le secteur Scarpe/Deûle/Escaut (Vitry-en-Artois, Noyelles-sous-Lens, Loison-sous-Lens et Valenciennes).

\section{LA VALLÉE DE LA SOMME AVAL}

Le site d'Étouvie « Le Chemin de la Marine » (Somme ; Prodéo et al. 1997) est implanté à la jonction d'une basse terrasse et du fond de la vallée alluviale de la Somme. Le site se développe en bordure d'un système de chenaux et de talwegs ayant permis un bon enregistrement environnemental. Ainsi, la séquence sédimentaire observée dans l'un des chenaux montre une corrélation entre un niveau charbonneux intercalé au sein $\mathrm{du}$ comblement tourbeux et l'installation d'une population BVSG (Deschodt in Prodéo et al. 1997, p. 46). Cette phase témoigne d'un important cycle de défrichements par le feu attribuable à une période comprise entre 5205 et 4860 cal. BC.

Onze occupations allant du Paléolithique final à l'époque moderne s'inscrivent au sein d'un niveau archéologique conservé autour d'une butte limoneuse. Les auteurs ont cherché à réduire "l'effet palimpseste » de cet horizon en cartographiant les découvertes de mobilier issu du niveau $(90 \%)$ et les structures sous-jacentes. Les résultats obtenus à partir des méthodes de fouille développées et les limites typologiques des assemblages sont discutés dans le rapport. Sans revenir sur ces débats, leur approche aura permis de reconstituer six ensembles chrono-culturels caractérisés par du mobilier issu du niveau et de quelques structures creusées. La phase du BVSG est identifiée à partir de trois fosses (ST 12-14 ; fig. 2, A) et d'un mobilier caractéristique de cet horizon (bracelet en schiste, céramique à cordon et lame en silex bartonien), provenant du niveau archéologique. Une sépulture se situe à proximité de cet ensemble. Elle contenait les restes d'un individu en position repliée sur le côté droit, les mains ramenées vers le visage, mais les nombreuses bioturbations et la mauvaise conservation de cet ensemble ne permettent pas une attribution chronologique fiable, même si un fragment de bracelet en schiste fut découvert en position remaniée à la hauteur du genou.

Ces trois fosses sont ovalaires à circulaires : 3,1 sur $1,8 \mathrm{~m}$ et 1 à $2 \mathrm{~m}$ de diamètre. Elles sont peu profondes (environ $0,15 \mathrm{~m}$ ). Elles ne sont pas considérées par les fouilleurs comme des fosses d'habitat car elles ne livrent qu'une très faible quantité d'artefacts. 


\begin{tabular}{|c|c|c|c|c|c|c|c|c|c|c|c|c|}
\hline 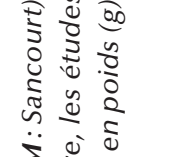 & 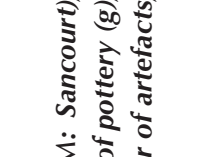 & 立 & & 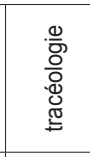 & & & & & 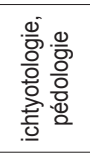 & 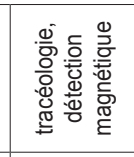 & & \\
\hline 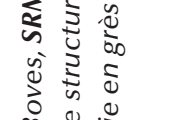 & 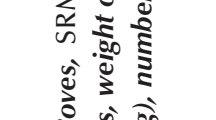 & & 递 & 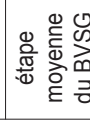 & & & & & 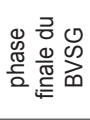 & 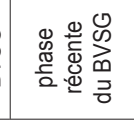 & 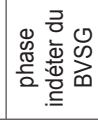 & 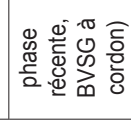 \\
\hline 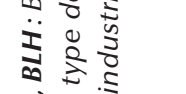 & $\ddot{\bar{I}}$ & $\begin{array}{l}\text { 喆 } \\
\text { 苋 }\end{array}$ & $\begin{array}{l}\frac{\circ}{\bar{g}} \\
\frac{0}{0}\end{array}$ & 흐 & ᄃे & ธ̄ & 호 & - & бо & ฮ̄ & б & ฮ̄ \\
\hline & 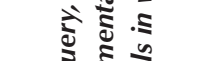 & & o & م & & & - & $r$ & - & $m$ & 흠 & 흗 \\
\hline & 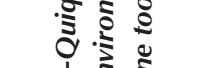 & & $\bar{\Sigma}$ & r & & & $\infty$ & & - & 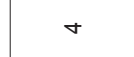 & - & \\
\hline 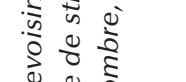 & 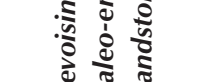 & 完 & $\frac{\mathscr{0}}{0}$ & $\stackrel{N}{N}$ & & & & & - & & & \\
\hline & ริำ & & 产 & § & & & & & - & $\stackrel{\circ}{\sim}$ & & - \\
\hline & & రั0 & $\frac{8}{0}$ & $\begin{array}{l}\text { 总 } \\
\stackrel{\circ}{\circ}\end{array}$ & & & & & - & & & \\
\hline & 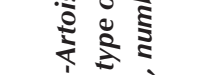 & & $\frac{\infty}{\underline{\underline{z}}}$ & 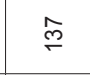 & & $m$ & & & $F$ & $\stackrel{\mathscr{B}}{\mathrm{n}}$ & $\approx$ & $\simeq$ \\
\hline & 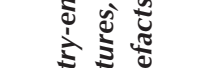 & $\frac{x}{\overline{\frac{\omega}{\omega}}}$ & $\frac{\grave{z}}{z}$ & $\stackrel{\infty}{\sim}$ & & $\stackrel{\circ}{\circ}$ & & & $\bar{c}$ & 总 & $\dddot{\varrho}$ & $\stackrel{\Re}{\sim}$ \\
\hline 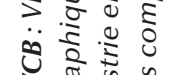 & & & $\frac{\mathscr{0}}{0}$ & 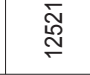 & & $\stackrel{m}{!}$ & & & $\stackrel{\text { In }}{\sim}$ & $\stackrel{\circ}{\circ}$ & $\stackrel{\infty}{i}$ & 足 \\
\hline & & & $\bar{\Sigma}$ & $\hat{\circ}$ & & $\simeq$ & o & & $\stackrel{\circ}{\circ}$ & F & & \\
\hline & & $\frac{\bar{z}}{\bar{c}}$ & $\frac{\hat{b}}{2}$ & & & & 応 & & $\stackrel{\infty}{\sim}$ & హ్ & $\bar{m}$ & 索 \\
\hline 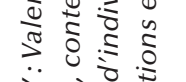 & 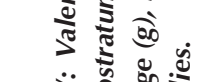 & $\circlearrowleft$ & $\frac{0}{0}$ & Оั & & $\stackrel{\infty}{\stackrel{f}{+}}$ & & & $\frac{0}{p}$ & 产 & 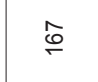 & $\stackrel{8}{\circ}$ \\
\hline & & 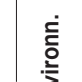 & 这 & $\stackrel{\infty}{\sim} \stackrel{\mathscr{e}}{\underline{\underline{\underline{e}}}}$ & б. & ㅎ & & Бे & $\stackrel{\circ}{\bar{m}}$ & $\stackrel{\overbrace{}}{\sim}$ & ธ & 흐 \\
\hline 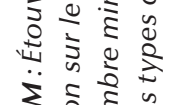 & 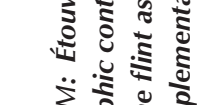 & 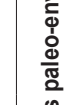 & 迡 응 & 호 & & & '̄ & 'े & б & ธ̄ & 흠 & б \\
\hline 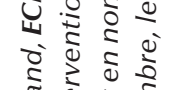 & 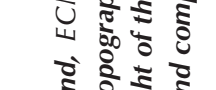 & 兽 & 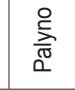 & 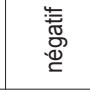 & 하 & ธ̄ & & 'े & б & ธ్ & 도 & 호 \\
\hline 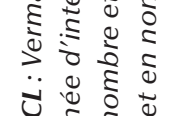 & 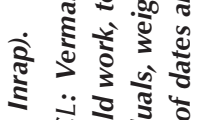 & 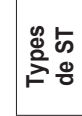 & & 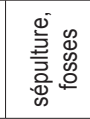 & & 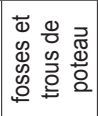 & 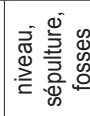 & & 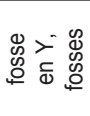 & 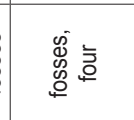 & 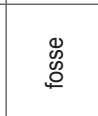 & 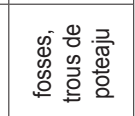 \\
\hline 8 & 等 & 竞占 & & $\stackrel{n}{\leftarrow}$ & $\sim$ & r & & 0 & $m$ & r & $r$ & $\infty$ \\
\hline 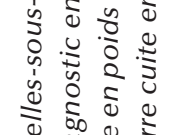 & 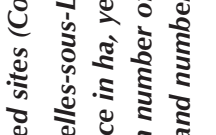 & 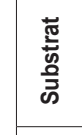 & & 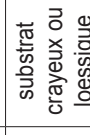 & & 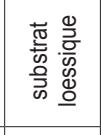 & & & 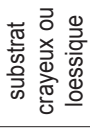 & 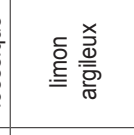 & 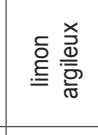 & $\begin{array}{l}\text { 을 } \\
\text { 을 } \\
\underline{\underline{\underline{w}}} \\
\frac{\bar{w}}{\sigma}\end{array}$ \\
\hline 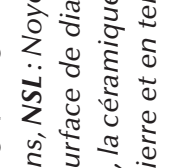 & 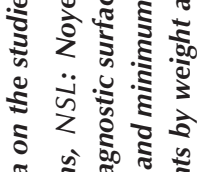 & 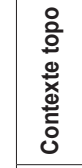 & & 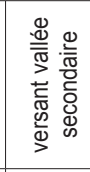 & & 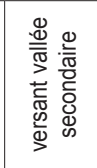 & 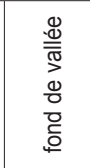 & & 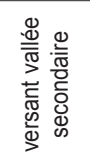 & 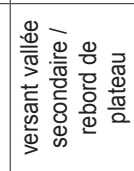 & 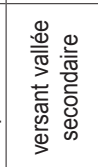 & 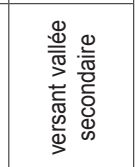 \\
\hline 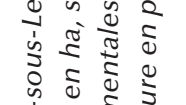 & 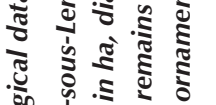 & 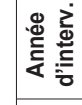 & & (a⿱丷丅) & & & 櫋 & & 宫 & 宫容 & $\stackrel{\infty}{\circ}$ & 옿음 \\
\hline & & 容 & $\frac{\pi}{1}$ & $\stackrel{\stackrel{n}{\sim}}{\sim}$ & $\stackrel{\infty}{\infty}$ & $r$ & & & o & & $\stackrel{0}{=}$ & $\bar{i}$ \\
\hline & $\frac{1}{9} \frac{1}{8}$ & 彭言 & 포 & $\stackrel{\infty}{=}$ & $\stackrel{f}{-2}$ & $\stackrel{\infty}{=}$ & & $\stackrel{8}{0}$ & $\because$ & $\stackrel{\infty}{-}$ & & \\
\hline$\underline{v}$ & 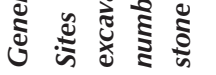 & $\stackrel{\mathscr{ٌ}}{\circ}$ & & $\vec{\omega}$ & $\overrightarrow{\underline{m}}$ & $\overrightarrow{\mathrm{S}}$ & 总 & $\sum_{>}^{+}$ & $\stackrel{\infty}{S}$ & g & 志 & $\sum_{\substack{c \\
c}}$ \\
\hline
\end{tabular}



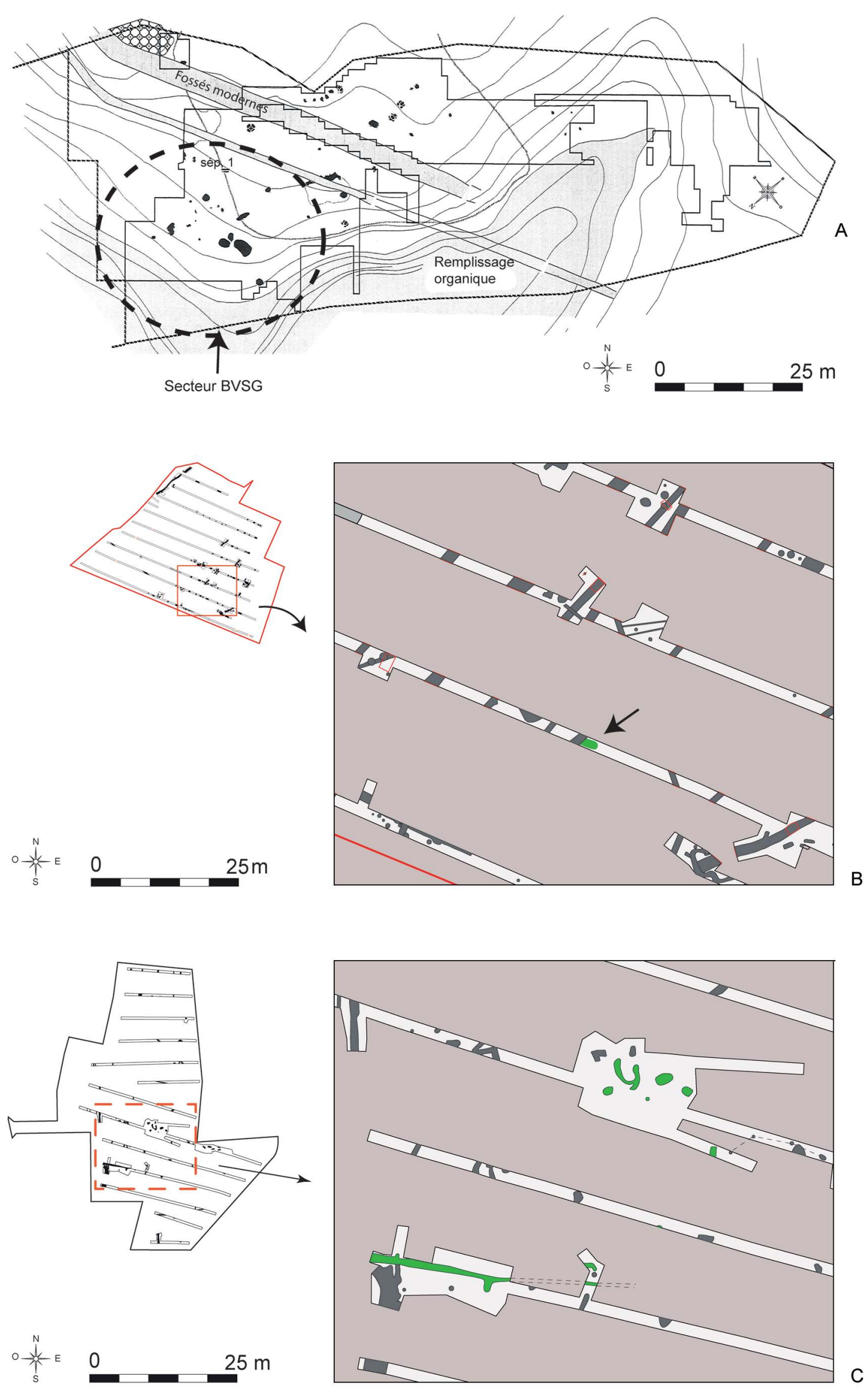
4 Fig. 2 - Les plans des occupations datées du Néolithique ancien dans la vallée de la Somme (structures du Néolithique ancien en vert sur $B$ et $C$ ).

A. Étouvie "Le Chemin de la Marine» (Somme; Prodéo et al. 1997) ; B. Boves "Les Longues Haies» (Somme ; Hébert 2009) ; C. Sancourt "Grande Rue/Rue du Moulin » (Somme ; Vacossin et al. 2010).

Land use during the Early Neolithic in the Somme valley (Ancient Neolithic structures in green on $B$ and $C$ ).

A. Étouvie "Le Chemin de la Marine" (Somme, Prodéo et al. 1997); B. Boves "Les Longues Haies" (Somme; Hébert 2009); C. Sancourt "Grande Rue/Rue du Moulin" (Somme; Vacossin et al. 2010).

Le mobilier retenu est faible par rapport aux quantités de matériaux siliceux récupérés mais nous avons privilégié les indices les plus caractéristiques qui orientent la datation de l'occupation vers une phase finale du BVSG. Nous nous appuierons donc uniquement sur les données et les dessins figurant dans le rapport final de l'opération.

Le site de Boves «Les Longues Haies » (Somme ; Hébert 2009) se situe sur le versant en rive gauche de la vallée de l'Avre, l'un des principaux affluents de la Somme. Le diagnostic réalisé en 2008 a permis la découverte d'une fosse attribuée au BVSG (fig. 2, B). La parcelle est située dans un petit vallon sec, un peu en retrait de sa confluence avec la vallée de l'Avre. Une implantation médiévale dense en vestiges est susceptible d'avoir freiné la reconnaissance des vestiges néolithiques. La fosse oblongue datée du Néolithique ancien est recoupée par un fossé postérieur. Sa longueur conservée est de $3 \mathrm{~m}$ pour une largeur de 1,50 m et une profondeur de 0,27 m. Creusée dans un limon argileux brun orangé, son comblement est constitué d'un limon brun gris comprenant quelques inclusions charbonneuses. Partiellement testée, cette structure a livré du mobilier céramique et lithique ainsi qu'un fragment de bracelet en schiste, le tout se rapportant à une phase récente du BVSG.

\section{LA VALLÉE DE LA SOMME AMONT}

La fouille du site de Languevoisin-Quiquery (fouille 20, secteur 3) menée dans le cadre du projet du canal SeineNord-Europe a porté sur un petit ensemble de sept fosses du Néolithique ancien réparties sur une surface de $1800 \mathrm{~m}^{2}$ (Baudry et al. 2013 ; fig. 3). Le site est localisé dans le nord-est de la Somme, sur le plateau du Santerre, plus précisément sur un versant surplombant l'Ingon. L'absence de trous de poteaux ne permet pas de reconstituer avec certitude la structuration du site.

L'état de conservation est très médiocre et toutes les fosses sont fortement arasées. Six d'entre elles sont des fosses de rejets, de forme subcirculaires ou ovalaires avec des longueurs maximales comprises entre $1,60 \mathrm{~m}$ et $2,70 \mathrm{~m}$. Les profondeurs oscillent entre 0,12 et $0,60 \mathrm{~m}$. Les creusements des fosses présentent dans leur ensemble des parois obliques, plus ou moins abruptes et un fond relativement plat ou en cuvette, avec peu ou pas de pendage. Leur comblement se partage généralement en deux grands types de remplissage : une zone charbonneuse,

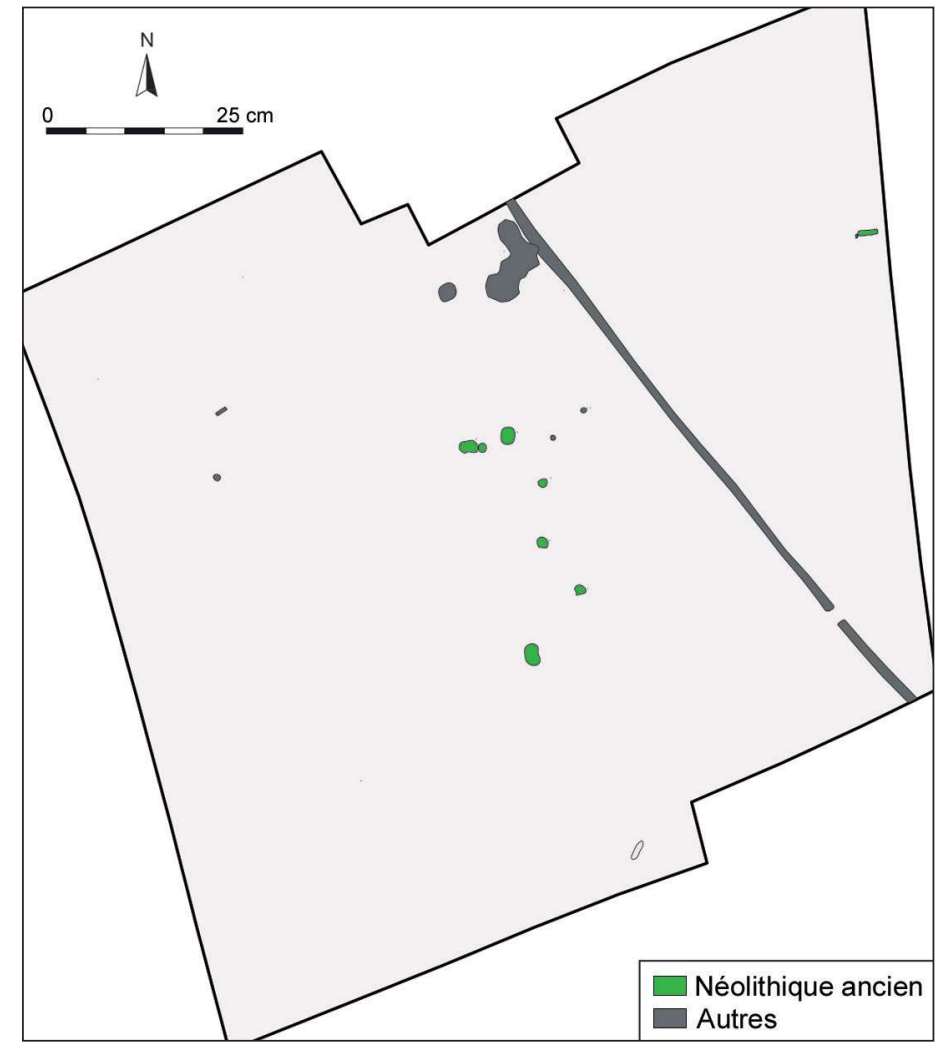

Fig. 3 - Plan de la fouille de Languevoisin-Quiquery "La Sole de Quiquery»(Somme ; Baudry 2013).

Languevoisin-Quiquery "La Sole de Quiquery" (Somme): excavation plan (Baudry 2013).

recueillant le matériel archéologique, et une zone d'interface avec l'encaissant, plus claire comportant peu ou pas de mobilier. Le comblement d'une fosse est plus complexe, car plusieurs couches charbonneuses se superposent et sont entrecoupées de limon proche de l'encaissant, illustrant par conséquent plusieurs phases de remplissage. La variété et la quantité de mobilier découvert (céramique, lithique et parfois de la parure) ainsi que les données des différentes études spécialisées identifient un lieu habité et exploité. La nature des rejets est semblable en de nombreux points à celle que l'on rencontre habituellement dans les fosses latérales des maisons danubiennes. Le rapport entre mobilier lithique et céramique est relativement constant et cohérent d'une fosse à l'autre. Ces structures, interprétées comme domestiques, étaient en outre accompagnées d'un four en fosse, type de structure qui se rencontre plus fréquemment au Cerny.

Le site de Vermand « Le Champ des Lavoirs » (Aisne ; Bostyn et al. 2003b) est implanté sur un versant limoneux en rive droite de la vallée marécageuse de l'Omignon, affluent de la Somme (fig. 4), au nord-est du département de l'Aisne. L'occupation principale concerne un établissement rural laténien se déployant sur 1,8 ha fouillé intégralement. Les indices d'une occupation du Néolithique ancien se caractérisent par une fosse (ST 1014) et un trou de poteau (ST 1157) localisés dans la partie interne de l'enclos gaulois tandis que le reste, neuf fosses et trois trous de poteau, se situe dans un 

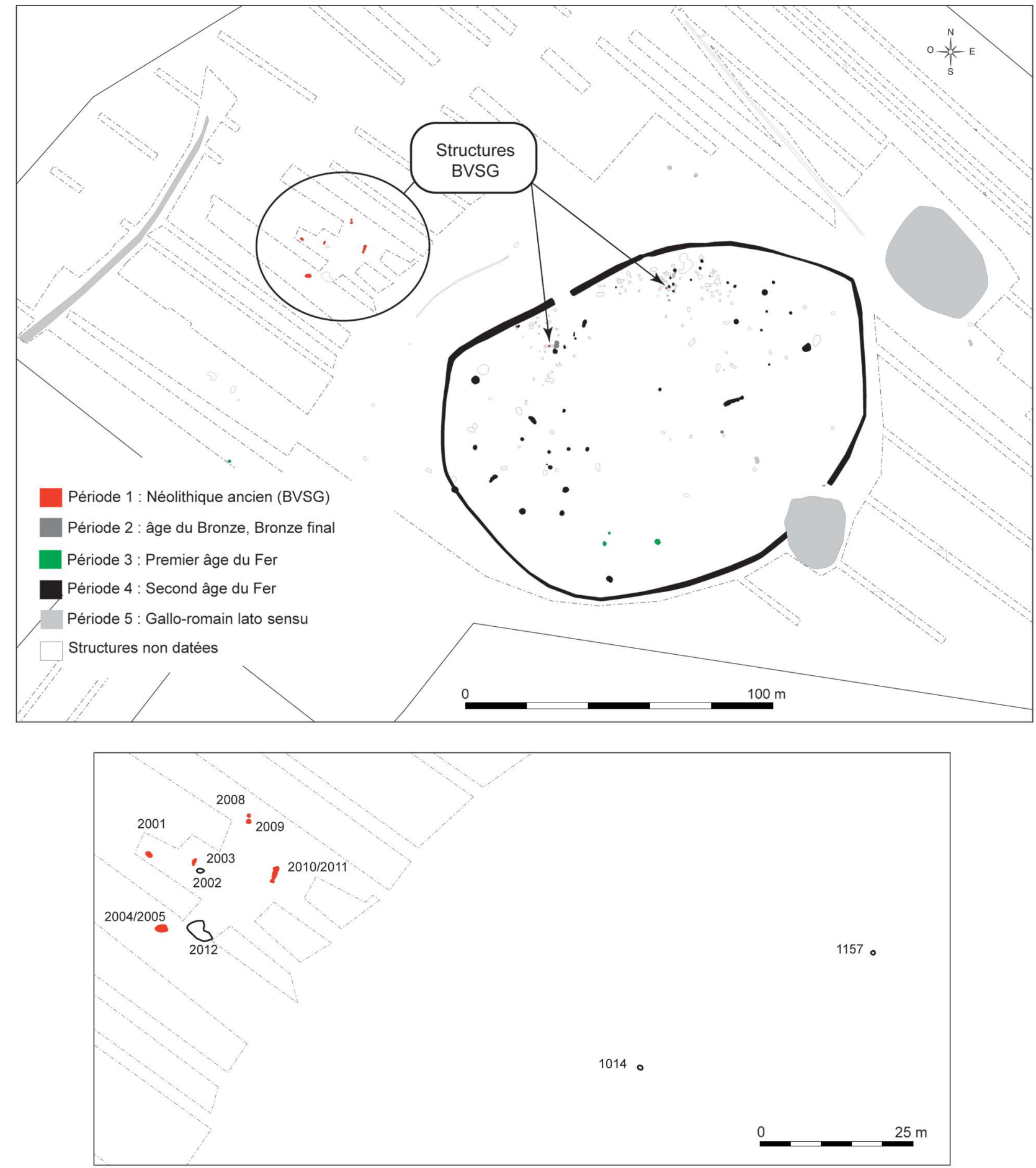

Fig. 4 - Vermand "Le Champ des Lavoirs" (Aisne). Plan général de la fouille avec un phasage des différentes périodes représentées, plus un agrandissement des ensembles datés du Néolithique ancien (Bostyn et al. 2003b). En rouge, les structures BVSG; en blanc, les structures associées.

The Early Neolithic site of Vermand "Le Champ des Lavoirs" (Aisne). General excavation plan with distinct periods and detailed dating (Bostyn et al. 2003b). In red, the BVSG structures; in white, the associated structures. 
second secteur traité en tranchées avec fenêtres de décapage. Les structures ont toutes fait l'objet d'une fouille exhaustive. Il s'agit de fosses ovalaires, peu profondes, en raison d'une érosion importante du site, livrant peu de mobilier lithique et céramique. Il n'y a pas d'organisation particulière et bien que la présence de trois poteaux structurés en tierce évoquerait les restes d'un habitat danubien, les arguments restent faibles.

À l'occasion d'un diagnostic réalisé en 2010 à Sancourt "Grande Rue/Rue du Moulin », des indices d'une occupation du BVSG et du Néolithique moyen ont été reconnus, au sein d'un site médiéval et moderne de plus grande ampleur (Vacossin et al. 2010 ; fig. 2, C). L'occupation est située sur un versant dominant la vallée de la Germaine, un affluent de la Somme en rive droite.

Cinq fosses et deux trous de poteau appartiennent au Néolithique ancien d'après le mobilier céramique et lithique. Réparties sur une surface de $3600 \mathrm{~m}^{2}$, les fosses sont creusées dans le limon et présentent une morphologie diverse, à tendance plutôt ovalaire. On note l'absence de véritables fosses oblongues évoquant les fosses latérales des bâtiments BVSG fréquents en fond de vallée. Le mobilier provient essentiellement d'un ramassage au niveau du décapage des fosses, hormis pour l'une des deux fosses dont une moitié a été fouillée manuellement. La céramique oriente l'attribution culturelle de cette occupation vers l'étape moyenne ou récente du BVSG. Le mobilier lithique reste compatible avec cette attribution. Des fragments de torchis ont également été recueillis.

\section{LES VALLÉES DE LA SCARPE, DE LA DEÛLE ET DE L'ESCAUT}

Le gisement de Valenciennes « Le Vignoble IV » (Nord) est implanté au pied d'un versant crayeux envahi par une tourbe au cours de l'Atlantique (Henton et al. 2009, Boulen et al. 2014). Une fenêtre de $540 \mathrm{~m}^{2}$ a été ouverte lors de la réalisation d'un bassin de réception des eaux pluviales à la jonction entre le pied du versant crayeux et le lit principal de l'Escaut au sud de la ville de Valenciennes (fig. 5). C'est un secteur où la vallée s'encaisse dans les derniers plateaux crayeux du Bassin parisien avant d'entamer sa course en direction des plaines sablo-limoneuses du Nord. Les informations livrées à cette occasion ne concernent que des données environnementales, aucun mobilier n'ayant permis de dater les différents horizons sédimentaires. En revanche, plusieurs coupes à la pelle mécanique d'une profondeur maximale de $4 \mathrm{~m}$ et des sondages à la tarière ont servi la reconstitution d'une séquence stratigraphique et pollinique couvrant l'Atlantique (8000 à 5000 BP). L'analyse de l'évolution morpho-sédimentaire couplée à un enregistrement pollinique et une série de datations radiocarbone ainsi qu'une étude dendrochronologique permettent de mesurer les variations du couvert forestier, de l'hydrométrie, de l'impact de l'activité humaine et des processus de sédimentation/érosion. Les prélèvements polliniques ont été effectués sur une hauteur de $3,5 \mathrm{~m}$ dont la partie supérieure $(1,5 \mathrm{~m})$ est organique et attribuable à l'Atlantique. Ces formations couvrent une durée estimée à plus de deux millénaires (6300-4000 av. J.-C.).
La chute d'un chêne $(0,9 \mathrm{~m}$ de diamètre) découvert dans la partie supérieure du colmatage tourbeux de l'Escaut, offre un indicateur chronologique pour étayer les observations environnementales. En effet, cet arbre comporte 268 cernes avec un dernier cerne (sans aubier) calé dans le courant de l'année 4930 av. J.-C (Dendrotech, Henton et al. 2009).

Le diagramme pollinique montre un milieu forestier dense du type chênaie mixte à tilleul sur toute la séquence Atlantique connaissant des phases de recul au profit d'essences héliophiles. Il révèle aussi la présence de plantes rudérales, de céréales ainsi qu'une augmentation des indices d'hygrométrie. Les variations de la présence du tilleul dans ce diagramme ont été rapportées pour partie à des modifications du taux d'humidité plutôt qu'à la présence de communautés humaines (Boulen et al. 2014). Il faut toutefois noter, que certaines rudérales témoignent de traces d'anthropisation à l'Atlantique récent (Néolithique ancien-moyen). Elles se caractérisent par l'apparition dans un premier temps de plantes rudérales seules entre 5053 et 4849 av. J.-C. (à 1 sigma) puis en association avec des céréales entre 4335 et 4257 av. J.-C.

Dans l'environnement immédiat de cet enregistrement, il n'y a pas de vestiges contemporains. Il faut alors se tourner en direction du bassin de Mons pour trouver les premiers témoins d'une occupation danubienne (Jadin 2003, p. 85). En effet, des indices sérieux de fréquentation sont observables dans la vallée de la Haine, affluent de l'Escaut et dont la confluence se fait à quelques kilomètres au nord de Valenciennes. Ils se caractérisent par la découverte de tessons céramiques décorés (Rubané) à Saint-Symphorien, de palet en schiste, d'armatures de flèche et d'herminette récoltés lors de prospections pédestres dans ce secteur (fig. 5, A). Ces vestiges témoignent d'une présence danubienne à quelques kilomètres à l'est de la vallée de l'Escaut.

Le site de Loison-sous-Lens « Parc d'activités des Oiseaux » (Pas-de-Calais ; Praud et al. 2010), est localisé dans la partie nord-est du Pas-de-Calais au cœur de la plaine limoneuse de Gohelle. Ce terroir s'appuie sur le soulèvement crayeux de l'Artois au sud et vient buter au nord sur les formations tertiaires du Pévèle, tandis qu'au nord-ouest il s'ouvre sur la plaine maritime de Flandre. L'occupation du Néolithique ancien a été reconnue à l'occasion de deux opérations de diagnostic menées successivement en 2003 (une fosse de rejet détritique) et en 2005 (six autres) sur une surface totale de 17 ha (Gaillard et al. 2003, Praud et al. 2005 ; fig. 6, A). Le site se développe sur le versant sud d'une butte limoneuse, à une altitude comprise entre 39 et $46 \mathrm{~m}$ (IGN 69). Globalement, ce terrain forme un léger replat avant de s'infléchir en pente douce vers le fond de la vallée du Souchez. À l'issue du décapage d'une surface de près de deux hectares (fig. 6, B), plusieurs ensembles ont été attribués, sur la base du mobilier, à la culture du BVSG : il s'agit de dix-huit structures, essentiellement des fosses de rejet détritique (13), quelques trous de poteaux (4) et une sépulture. Elle contenait un individu allongé sur le dos dont les membres inférieurs sont repliés sur le côté droit avec un petit vase déposé à proximité du genou gauche et le membre supérieur droit également replié. La répartition spatiale des creusements est relativement lâche et le faible nombre de trous de poteaux 
Fig. 5 - Valenciennes «le Vignoble» (Nord; Henton et al. 2009, Boulen et al. 2014).

\section{Localisation}

2. Situation dans la vallée de l'Escaut : A. plaine alluviale de I'Escaut, B. lit majeur de la Rhonelle, C. tracé présumé du talweg, 1. site du Vignoble ;

3. Log synthétique des prélèvements palynologiques et dates radiocarbone (se reporter au tabl. 11 pour le détail) ; unité $C$ : limon lité sablo-graveleux (Pléistocène), unité $\boldsymbol{D}$ : sol noir (Tardiglaciaire Allerǿd ?), unité $\boldsymbol{E}$ : silex matrice limoneuse (dépôt grossier au pied du versant, Dryas récent ?), unité $\boldsymbol{F}$ : sable et limon plastique (colluvion en pied de versant, début Holocène), unité $G$ : Tourbe (Atlantique), unité $I$ : sol noir (subatlantique), unité $J$ : limon, silex, structures (remaniements anthropiques récents).

Valenciennes "Ie Vignoble" (Nord; Henton et al. 2009, Boulen et al. 2014).

1. Location;

2. Situation in Scheldt valley: A. Alluvial plain of the Scheldt, B. Major bed of the Rhonelle valley, C. Supposed Talweg line, 1. Vignoble site; 3. Synthetic Log with palynological samples and radiocarbon dates; unit C: Sandy-gravelly bedded silt (Pleistocene), unit D: Dark soil (Allerǿd Tardiglacial?), unit E: Flint silty matrix (coarse deposit at the base of the slope, recent Dryas?), unit F: Sand and plastic silt (colluvium at the base of the slope, early Holocene), unit G: Peat (Atlantic), unit I: Dark soil (subatlantic), unit J: Silt, flint, digging (recent human alteration).

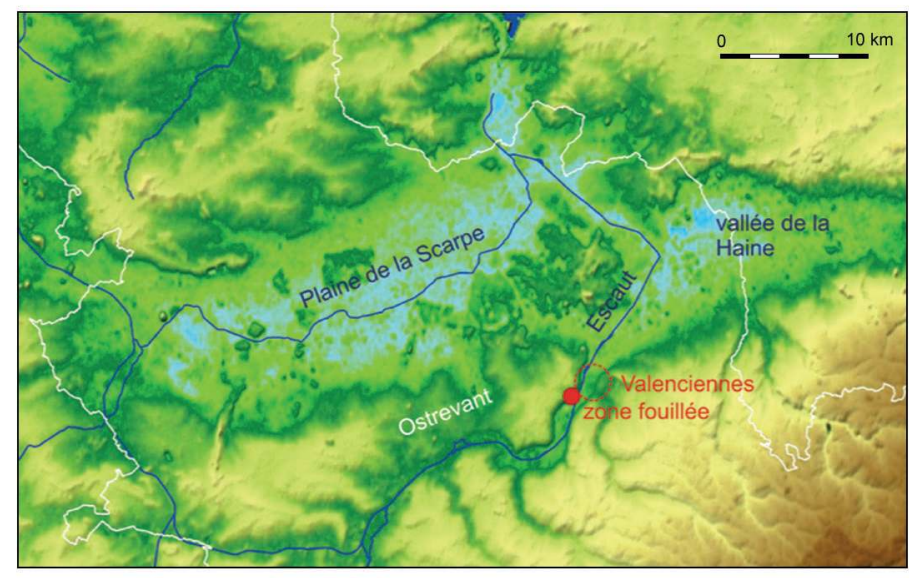

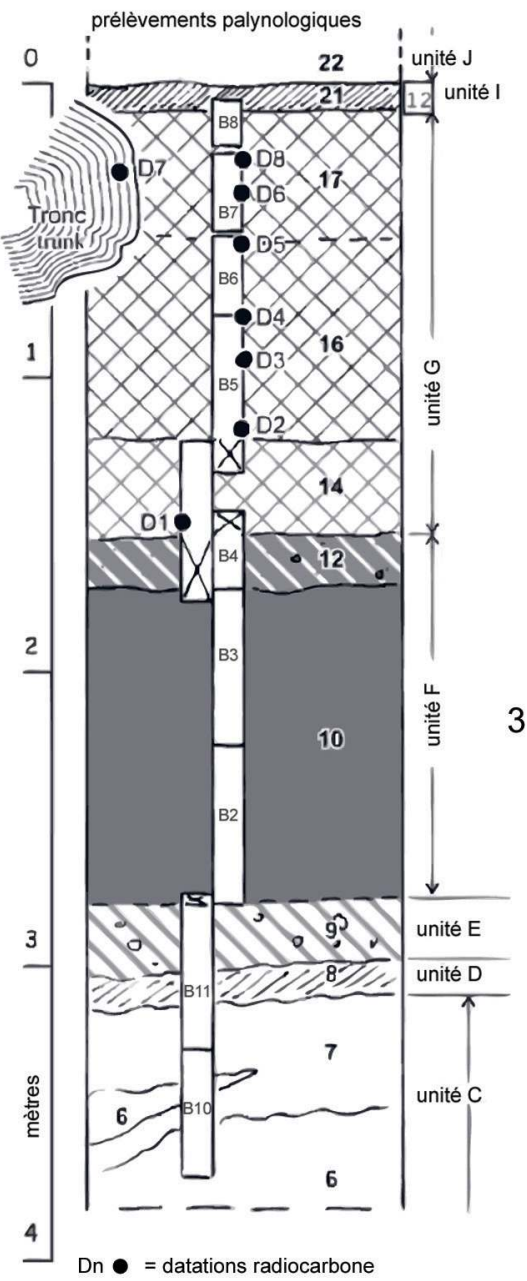
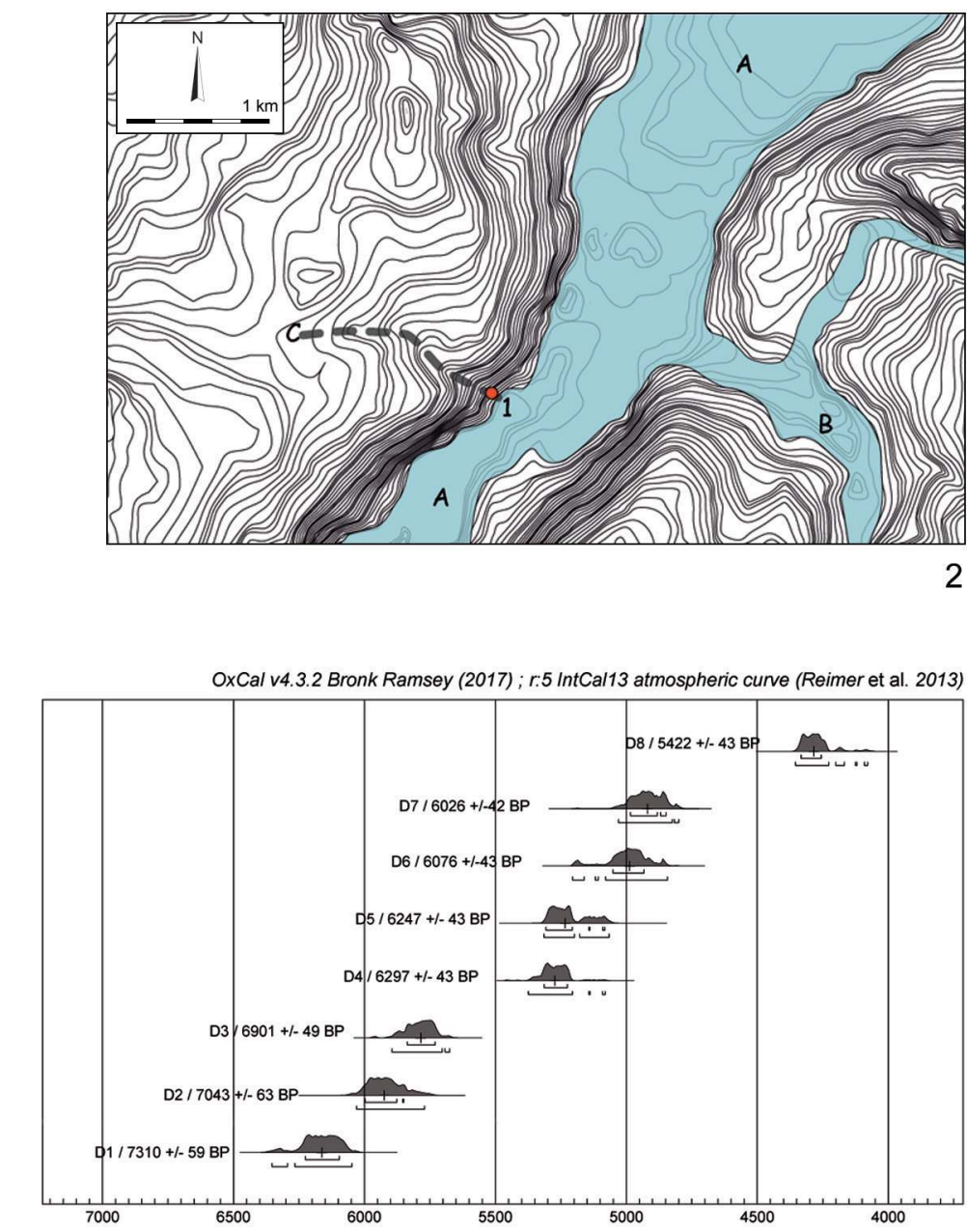

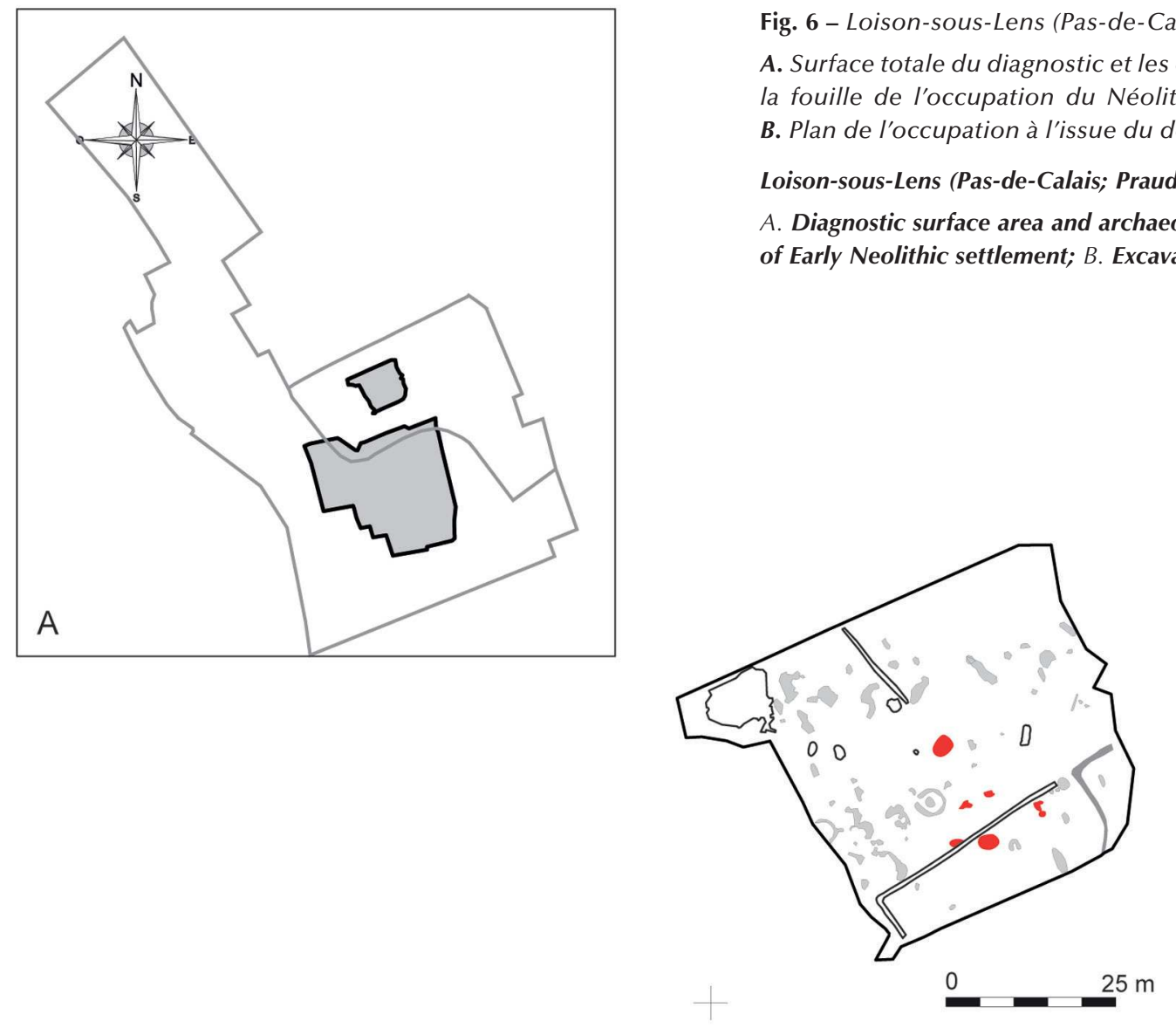

Fig. 6 - Loison-sous-Lens (Pas-de-Calais; Praud et al. 2009a).

A. Surface totale du diagnostic et les emprises prescrites pour la fouille de l'occupation du Néolithique ancien en grisé ; B. Plan de l'occupation à l'issue du décapage.

Loison-sous-Lens (Pas-de-Calais; Praud et al. 2009a).

A. Diagnostic surface area and archaeological excavated surface of Early Neolithic settlement; B. Excavation plan.

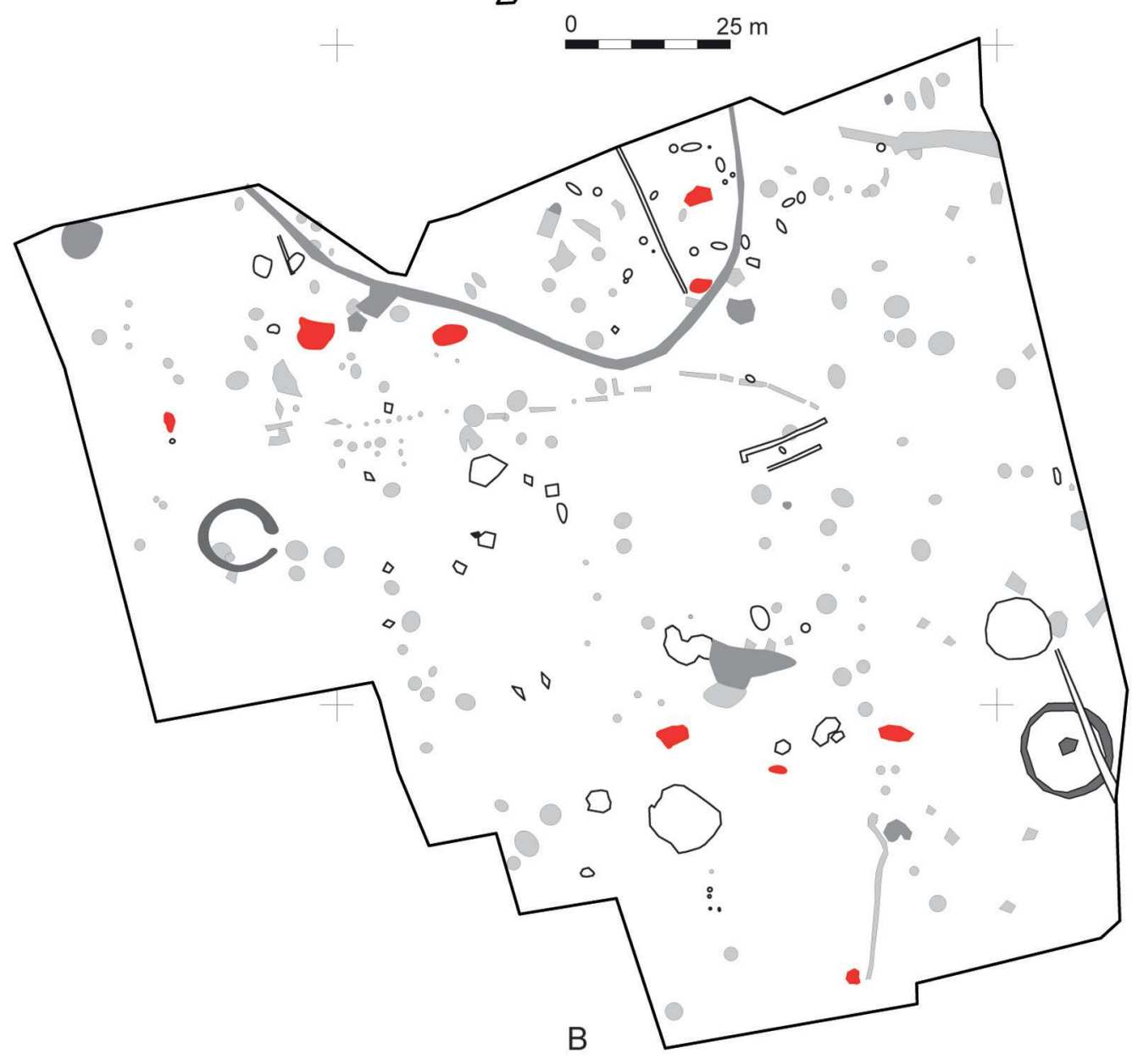


constitue un frein à l'interprétation du site en termes d'unités d'habitation. Toutefois, l'organisation des creusements sur un axe globalement est/ouest évoque l'agencement des fosses latérales qui structurent en général la maison « danubienne ».

À Noyelles-sous-Lens « Parcelle Bertelsmann » (Pas-deCalais ; Hosdez 2001), 2,5 hectares ont été fouillés sur les six diagnostiqués. L'emprise décapée révèle de nombreux impacts d'obus datant de la première guerre mondiale, une occupation principale antique, ainsi qu'une fosse (ST 700) ovalaire de 1,6 sur $1 \mathrm{~m}$ et $0,60 \mathrm{~m}$ de profondeur datée du Néolithique ancien. Ce gisement se localise en rive droite de la vallée de la Souchez à moins d'1 km du site de Loison-sous-Lens.

Le site de Vitry-en-Artois « Chemin-Brûlé » (Pas-de-Calais) a été fouillé en 2008, sur une emprise de $6600 \mathrm{~m}^{2}$ comprenant des vestiges fugaces d'une occupation BVSG (Cayol et al. 2015). Le site est implanté sur le versant d'une butte crayeuse à proximité de la plaine alluviale récente de la Scarpe, à $15 \mathrm{~km}$ à l'est d'Arras. L'occupation se résume à trois structures dont une fosse en « $\mathrm{Y}$ » ayant livré la majeure partie du mobilier et deux petites fosses très arasées, chacune d'elles offrant un mobilier plutôt indigent. La datation du mobilier céramique renvoie à l'étape finale du BVSG, étape qui n'avait pas été documentée jusqu'ici. Les études paléoenvironnementales (carpologie, ichtyologie) et micromorphologiques n'ont pas apporté d'indices déterminants concernant la fonction de ce type de fosse. L'étude carpologique a mis en évidence la présence de végétaux évoluant dans des sédiments hydromorphes. On note la présence du tilleul, essence bien connue pour l'utilisation du liber dans l'artisanat textile. Pourtant, l'hypothèse d'une utilisation comme fosse de rouissage est plutôt infirmée par les résultats de l'étude micromorphologique : on ne note ni battement de la nappe ni problème de drainage. Plusieurs restes de poissons témoignent d'activités de pêche.

Enfin, il reste à signaler le site de Blangy-sur-Bresle (Seine-Maritime), souvent cité comme appartenant au BVSG (Daniel 1973, Constantin et Ilett 1982) et localisé en rive gauche de la Bresle, sur une éminence limoneuse. Les fouilles anciennes et les ramassages hors contexte ont mis au jour une série lithique très abondante et un corpus céramique plus restreint au sein duquel « des tessons décorés de chevrons organisés en bandes verticales et des tessons dégraissés à l'os pilé » sont attribués au BVSG (Constantin et Ilett 1982, p. 126). Le gisement a livré aussi un ensemble céramique proche des séries de Catenoy dans l'Oise daté du Chasséen. Sans s'attarder sur cet assemblage, visiblement hétérogène, nous ne retiendrons finalement de ce site qu'un point géographique situé au sud de la vallée de la Somme, à une trentaine de kilomètres d'Abbeville.

\section{INTERPRÉTATION DES SITES}

Les gisements présentés précédemment constituent donc un ensemble assez hétéroclite de sites où l'absence de plan de maison ne facilite pas l'interprétation. Par ailleurs, les creusements présentent des morphologies différentes, et partagent quasiment tous un état de conservation médiocre (mis à part les structures 11 et 1008 du site de Loison-sous-Lens) indiquant probablement une érosion importante et des perturbations liées aux occupations postérieures. Traditionnellement, le modèle d'habitat dit danubien, reconnu sur une vaste aire géographique, comporte des fosses allongées bordant les longs côtés de maisons trapézoïdales orientées généralement sur un axe est/ouest et fondées sur poteaux de bois (Coudart 1998). Plusieurs études récentes ont montré que non seulement les sites d'habitats Rubané final et BVSG du Bassin parisien livraient des types de structures variés et pas seulement des fosses latérales (Meunier et al. 2014), mais aussi que certaines occupations étaient matérialisées uniquement par des fosses de morphologies très différentes des fosses latérales, qui étaient dispersées dans l'espace et en cela ne correspondaient pas à la définition stricte d'un habitat (Lanchon 2012). Les fosses « isolées », localisées en rebord de plateau ou en périphérie des zones alluviales dans le Bassin parisien (Lanchon et al. 2005), sont souvent subcirculaires avec un comblement stratifié et représentées en deux ou trois exemplaires maximum. On peut citer l'exemple des sites de Vignely « les Hayettes » (Brunet et Lanchon 2002), de Mareuil-les-Meaux « RSOM » (Durand 2004) ou de Villenoy « les Seize Arpents » (Durand 2007). Ainsi, en l'absence ou presque de poteaux, il convient de se pencher sur la morphologie, les remplissages et l'agencement des structures pour proposer une interprétation des gisements, tout au moins ceux qui ont fait l'objet d'une fouille.

Les fosses peuvent se diviser en quatre principales catégories de forme (fig. 7) :

- A. Les très grandes fosses qui possèdent une longueur supérieure à $3 \mathrm{~m}$, des contours irréguliers, une faible profondeur et des parois évasées. Elles présentent toutes des alvéoles de surcreusement de plus petites dimensions ;

- B. Les fosses plus longues que larges mais de dimensions inférieures aux précédentes avec un diamètre se situant autour de $3 \mathrm{~m}$, dessinant des contours irréguliers, comportant des profils de creusement évasés. Elles sont légèrement plus profondes que les précédentes, un cas de surcreusement évident est notamment observable dans la structure 11 à Loison-sous-Lens ;

- C. Les plus nombreuses dans notre corpus sont des fosses dont les contours sont plus réguliers que les précédents creusements. Quelques exemplaires sont toujours un peu plus longs que larges mais l'essentiel est de forme circulaire avec un diamètre situé principalement autour de $2 \mathrm{~m}$ et des parois plutôt verticales. Les profondeurs peuvent atteindre près de $0,80 \mathrm{~m}$;

- D. Les structures atypiques intéressent principalement les creusements à profil en « $\mathrm{Y}$ ». Elles sont représentées sur deux gisements, Languevoisin-Quiquery et Vitry-en-Artois.

Il est difficile, à partir de ces témoignages bruts, de déterminer la fonction première de ces creusements. Ainsi, pour essayer de statuer sur la nature des occupations, on peut tenter de positionner des plans complets de bâtiments y compris leurs fosses sur les vestiges conservés. Nous avons pris comme point 

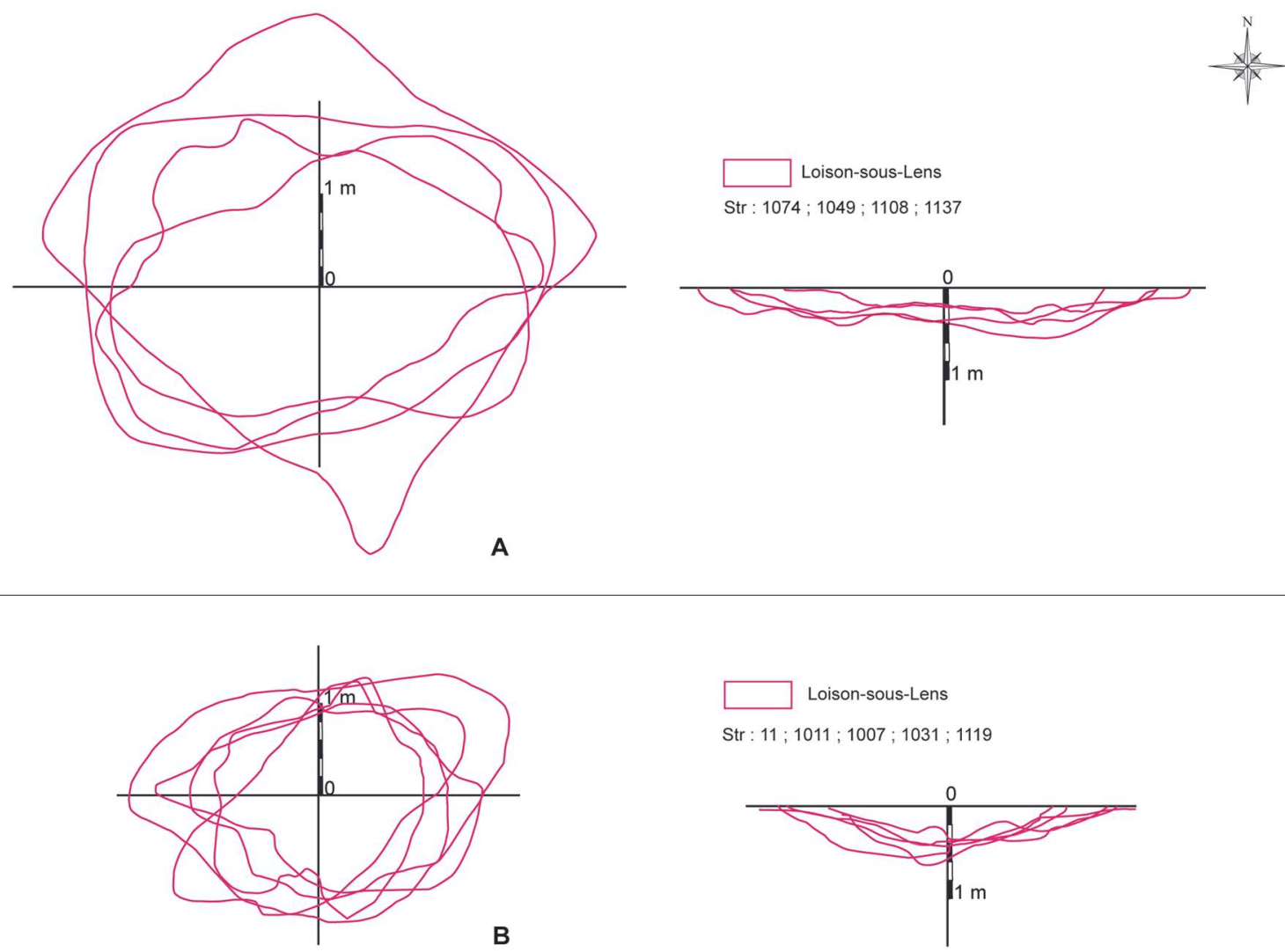

Loison-sous-Lens

Str : $11 ; 1011 ; 1007 ; 1031 ; 1119$
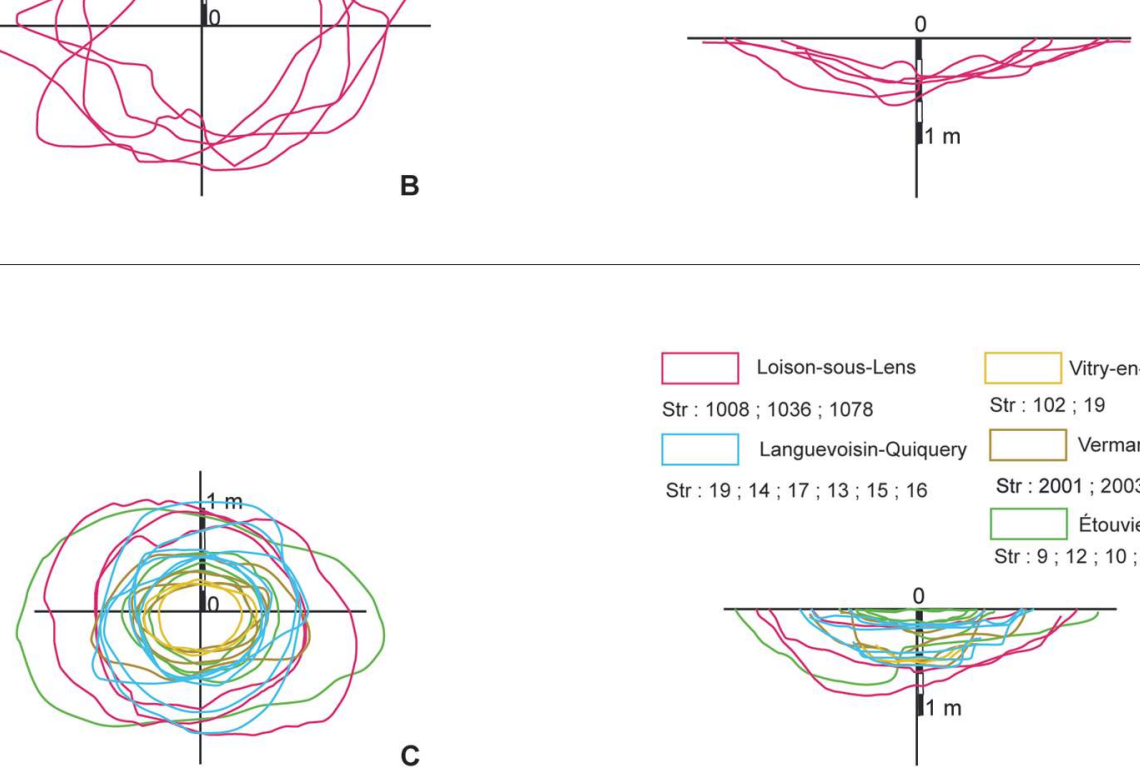

Loison-sous-Lens

Str : $1008 ; 1036 ; 1078$

Languevoisin-Quiquery

Vitry-en-Artois

Str : $19 ; 14 ; 17 ; 13 ; 15 ; 16 \quad$ Str : $2001 ; 2003 ; 2004 ; 2009$

$\square$ Étouvie

Str : $9 ; 12 ; 10 ; 11$
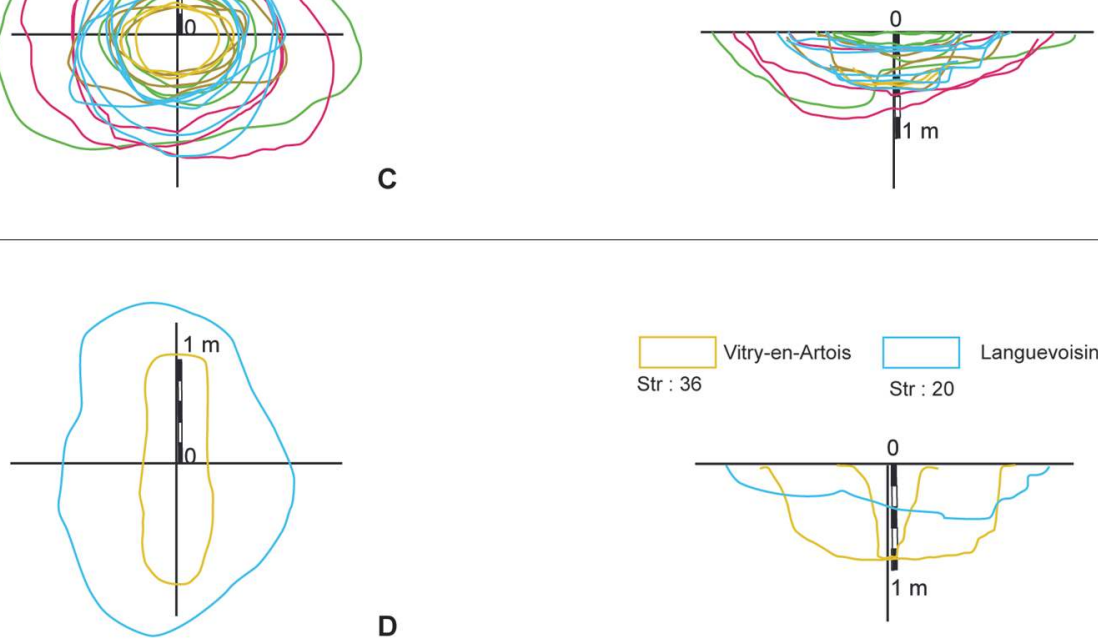

D
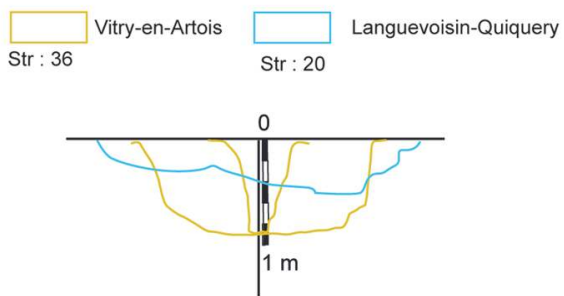

Fig. 7 - Les différentes catégories morphologiques en plan et en coupe des fosses du Néolithique ancien BVSG (I. Praud, Inrap). The different morphological categories of Early Neolithic BVSG pits cross-sections and plane views (I. Praud, Inrap). 
de comparaison le site le plus proche des implantations de notre secteur d'étude et le mieux structuré d'Irchonwelz « La Bonne Fortune » (Constantin 1985) localisé dans le Hainaut belge.

Le site de Loison-sous-Lens se prête plutôt bien à ce type d'exercice (fig. 8). La concentration dans le secteur nord du décapage pourrait constituer les restes d'une unité d'habitat avec une répartition des creusements entre fosses nord et sud (ST 11 et 1008-1007 ; fig. 8). Ces deux fosses ont par ailleurs livré une quantité importante de mobilier archéologique et notamment quatre meules complètes en grès pour la seule structure 11 ainsi que des fragments d'outils de mouture dans la structure 1008. Elles font partie des creusements les mieux conservés de ce gisement. Si l'espace entre ces deux creusements $(15 \mathrm{~m})$ est supérieur à ceux observés en général, la profondeur de ces fosses $(0,60$ à $0,80 \mathrm{~m})$ témoignerait d'une conservation partielle de fosses de plus grandes dimensions au départ. Quelques structures occupent la partie interne. Il s'agit du creusement 1007 recoupé légèrement dans sa partie sud par un fossé protohistorique (ST 1006). Moins profonde et aux parois plus évasées, elle a livré un mobilier moins riche mais attribuable à l'occupation BVSG. La fosse 1011 présente, elle, une succession stratigraphique très perturbée et procure peu de mobilier rendant l'ensemble assez discutable; seuls les contours d'un trou de poteau justifieraient son maintien ici. Il en est de même des restes de probables trous de poteaux dont les comblements et les contours sont mal définis, parfois perturbés par des terriers. Quelques creusements dans ce secteur s'apparentent à des restes de trous de poteaux, mais ils trouvent difficilement une place cohérente dans ce type d'architecture.

Concernant la plus grande emprise décapée, les assemblages entre de possibles fosses nord et sud semblent plutôt bien fonctionner à condition d'accepter que seule une partie des creusements soit conservée.

Si cet exercice peut apparaître comme peu démonstratif, la nature des vestiges, la distribution spatiale des fosses sur un axe nord-est/sud-ouest, la morphologie des creusements et enfin la superposition des creusements d'une unité d'habitat permettent de poser comme hypothèse qu'il s'agit bien à Loison-sousLens d'un site d'habitat. La présence d'une sépulture constitue un autre argument pour favoriser cette hypothèse puisque les seules sépultures connues à ce jour pour le BVSG sont localisées à proximité immédiate des maisons (Thévenet 2010).

Pour le site de Vermand, l'agencement des fosses et des quelques poteaux avait favorisé l'hypothèse de l'existence d'au moins une unité d'habitation mal conservée (Bostyn et al. 2003b).

Concernant le site de Languevoisin-Quiquery, l'orientation des fosses n'est pas très marquée, du fait de leur forme quasiment circulaire. En revanche, plusieurs d'entre elles présentent des alignements nord-est/sud-ouest. Si les distances nord-sud entre certaines fosses ne sont pas sans rappeler la largeur des maisons BVSG, la disposition des fosses ne permet pas de reconstituer de manière évidente des unités d'habitation et elle ferait plutôt penser à des lieux d'activités temporaires situés à proximité d'un habitat.

À Vitry-en-Artois, la dispersion des fosses, leurs morphologies et la composante mobilière sont autant d'arguments qui ne plaident pas non plus en faveur d'une interprétation du site

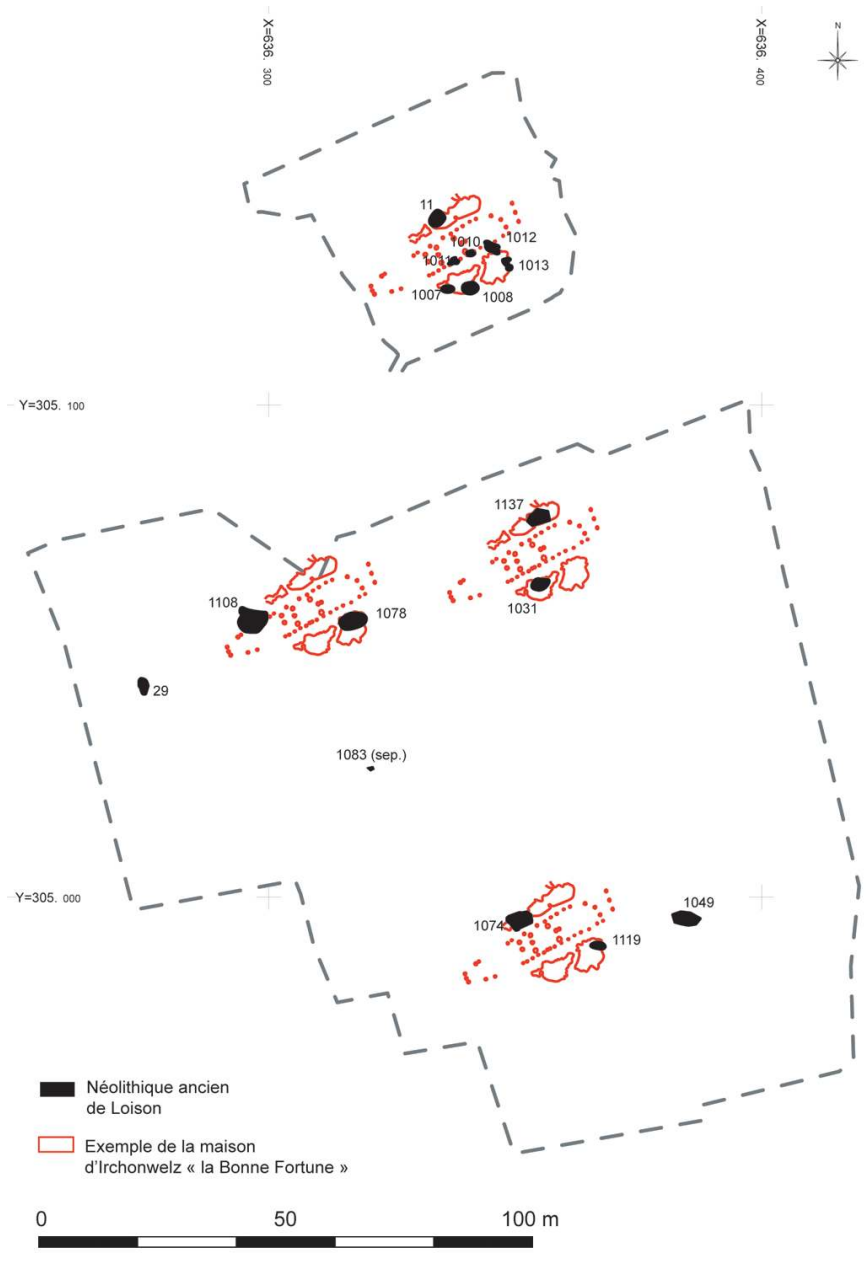

Fig. 8 - Superposition de la maison du site d'Irchonwelz (Belgique) sur les structures du site de Loison-sous-Lens (d'après Constantin 1985 ; DAO P. Ladureau et I. Praud, Inrap).

Superposition of house plans at the Irchonwelz settlement (Belgium) and Loison-sous-Lens pits (after Constantin 1985; CAD P. Ladureau and I. Praud, Inrap).

comme un habitat, mais plutôt comme une occupation ponctuelle peut-être liée à une activité particulière.

Pour le site d'Étouvie enfin, même si l'hypothèse d'une unité d'habitation n'a pas été retenue par les fouilleurs (Prodéo et al. 1997), la disposition des quelques fosses et la présence d'une sépulture pourraient quand même révéler la présence des restes d'un habitat limité.

En résumé, notre corpus ici constitué comprend deux sites d'habitat (Loison-sous-Lens, Vermand), possiblement un troisième à Étouvie, un site en bordure immédiate d'un habitat (Languevoisin-Quiquery) et une occupation non domestique (Vitry-en-Artois). Pour les autres ensembles (Boves, Sancourt, Noyelles-sous-Lens), il n'est pas possible de se prononcer en l'état actuel de nos connaissances.

Ainsi, ces conclusions rejoignent les travaux de recherche dirigés par Y. Lanchon (2012) qui ont montré, à partir de corpus plus étoffés issus de la basse vallée de la Marne, comment les populations BVSG pouvaient structurer et aménager les 
espaces en dehors des villages à proprement parler. Les sites à fosses, dont les formes sont différentes de celles rencontrées sur les sites d'habitat (morphologie circulaire et profonde, remplissage complexe...), sont découverts à distance des villages et apparaissent isolés. Dans le Bassin parisien, les villages à longue durée d'occupation sont installés dans les fonds de vallée tandis que ces fosses « isolées » sont localisées sur des terrains limoneux des plateaux propices à la culture et sont situées à environ $1 \mathrm{~km}$ de rayon autour des habitats pérennes. Ces gisements satellites semblent se répartir selon les besoins économiques de la communauté ce qui paraît tout à fait normal mais difficile à démontrer. Il convient donc d'intégrer à cette discussion les informations que peuvent nous apporter les différentes catégories de mobiliers.

\section{LES PRODUCTIONS MATÉRIELLES}

\section{LA CÉRAMIQUE}

Cinq sites ont été retenus pour l'analyse de la céramique, il s'agit de Loison-sous-Lens, Vitry-en-Artois, LanguevoisinQuiquery, Étouvie et Vermand. La série compte 148 individus pour un poids total de $18,7 \mathrm{~kg}$ dont un tiers environ porte un décor (tabl. 2 et fig. 9). La représentativité du nombre moyen d'individus céramique par site est de 30 exemplaires et la comparaison avec d'autres ensembles régionaux témoigne de la faiblesse de notre corpus. En effet, il se place nettement au-dessus de séries pauvres comme celles recensées dans le Centre de la France avec sept individus-vases par site (Creusillet et Irribarria 2008), mais nettement en dessous des ensembles très riches du Bassin parisien comme ceux de la vallée de la Marne qui offre près de 127 individus par site (Lanchon 2008). Les écarts relatifs avec les séries voisines du groupe de Blicquy, dont le nombre de vase est estimé à 50 individus par site, sont moins importants (Hauzeur 2008).

La réalité est encore plus marquée lorsque l'on s'intéresse à la répartition des données entre les gisements puisque Loisonsous-Lens livre pratiquement la moitié du corpus dans tous les registres (en poids, NMI et vases décorés). On observe, en outre, une distribution intrasite disparate entre les structures. À Loison-sous-Lens, par exemple, trois structures sur treize regroupent $70 \%$ du mobilier en terre cuite. De la même manière, le mobilier céramique de Vitry-en-Artois ne provient que d'une seule structure (ST 36) tandis que la majorité des vases $(65 \%)$ de Languevoisin-Quiquery résulte de la fouille de deux fosses sur les sept attribuées au BVSG.

D'une façon générale, la céramique n'est pas très bien conservée, les surfaces sont érodées, de sorte que les décors les moins profondément incisés mais surtout imprimés sont souvent difficiles à lire et à interpréter. La faible température de cuisson rend aussi cette céramique particulièrement fragile.

Tabl. 2 - Données en poids, en NMI, répartition du nombre de vases décorés/non décorés, types de dégraissants et couleur de cuisson (interne / à cœur / externe ; C : claire, $\boldsymbol{F}$ : foncée) du corpus céramique provenant des gisements étudiés (I. Praud, Inrap).

Data by weight, MNI, distribution of the number of decorated /non-decorated pottery vessels, types of temper, and firing colour (internal/centre/external; C: light-coloured, F: dark-coloured) of the pottery from all the studied (I. Praud, Inrap).

\begin{tabular}{|c|c|c|c|c|c|c|c|c|c|c|c|c|c|c|}
\hline \multirow[t]{2}{*}{ Sites } & \multirow[t]{2}{*}{ Poids } & \multirow[t]{2}{*}{ NMI } & \multirow[t]{2}{*}{ Décoré } & \multirow[t]{2}{*}{ Non décoré } & \multicolumn{5}{|c|}{ Dégraissants } & \multicolumn{5}{|c|}{ Cuisson } \\
\hline & & & & & Calcaire & Os & Sable & Coq & Chamotte & CFC & $\mathrm{CCC}$ & FFF & FFC & CFF \\
\hline LSL & 8092 & 67 & 23 & 44 & 5 & 20 & 15 & 2 & 2 & 38 & 7 & 5 & 1 & 7 \\
\hline VCB & 3130 & 16 & 8 & 8 & 3 & 9 & 1 & & 0 & 4 & 3 & 2 & 1 & 3 \\
\hline VCL & 1448 & 12 & 3 & 9 & 3 & 1 & 5 & 0 & 0 & & 3 & 3 & & 5 \\
\hline $\mathrm{LQ}$ & 4841 & 44 & 8 & 36 & 17 & 3 & 24 & 0 & 0 & 24 & 2 & 2 & & 14 \\
\hline ECM & 1200 & 9 & 5 & 4 & & & & & & & & & & \\
\hline Total & 18711 & 148 & 47 & 101 & 28 & 33 & 45 & 2 & 2 & 66 & 15 & 12 & 2 & 29 \\
\hline
\end{tabular}

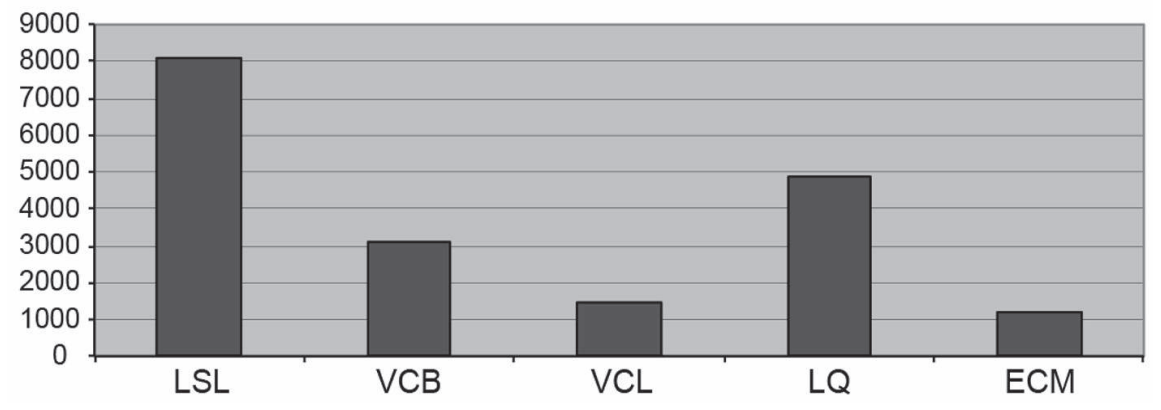

Fig. 9 - Répartition en poids (g) par site des restes céramiques (I. Praud, Inrap).

LSL : Loison-sous-Lens (62), VCB : Vitry-enArtois (62), VCL : Vermand (02), LQ : LanguevoisinQuiquery (80), ECM : Amiens "Étouvie » (80).

Weight distribution of pottery by site (I. Praud, Inrap). LSL: Loison-sous-Lens (62), VCB: Vitry-en-Artois (62), $V C L$ : Vermand (02), LQ: Languevoisin-Quiquery (80), ECM: Amiens "Étouvie" (80). 


\section{LA TECHNOLOGIE}

Globalement, les individus céramiques de ce corpus présentent des caractères techniques communs. Quatre sites sont suffisamment étudiés pour permettre une analyse technologique. Trois types de dégraissant sont utilisés principalement : le calcaire, l'os pilé et brûlé, et le sable (fig. 10, A). L'ajout volontaire de sable dans la préparation de la pâte doit être discuté car sans un examen précis de la granulométrie, il est difficile de trancher entre le choix d'une argile dégraissée naturellement et l'ajout volontaire d'une fraction sableuse criblée et triée. Néanmoins, la reconnaissance d'éléments sableux sur tous les sites et sa place prépondérante dans ce corpus inclinent à penser qu'il s'agirait bien d'un ajout volontaire.

Ensuite, par ordre d'importance, on trouve à parts égales l'os brûlé et pilé, et le calcaire, tous deux incorporés dans la préparation de la pâte. Enfin, l'utilisation de la chamotte et de la coquille est très anecdotique, on l'observe uniquement sur le site de Loison-sous-Lens.

Dans le détail, il existe des différences entre les gisements de Loison-sous-Lens et de Vitry-en-Artois, où l'os est dominant (fig. 10, B), et ceux de Languevoisin-Quiquery et Vermand où le sable et le calcaire sont majoritaires. L'utilisation importante de l'os calciné pilé, plutôt rare dans le Bassin parisien, renvoie plutôt aux pratiques du groupe de Blicquy, où son utilisation est plus fréquente (Constantin 1985).

La couleur des surfaces externes est très majoritairement à dominante brune à brun-rouge (fig. 11, A) et le cœur de la paroi des tessons est d'un noir plus ou moins intense témoignant d'une cuisson des vases en atmosphère réductrice. La fin de la cuisson révèle bien souvent une phase oxydante $(53 \%)$ qui peut être totale $(12 \%)$ ou à l'inverse ne concerner que la surface externe $(23 \%)$. Cette variabilité dans les techniques de cuisson ne se dément pas sur chaque site (fig. 11, B).

Les cassures sur joint de colombin sont les plus fréquentes attestant de techniques de montage classiques dans ce type de corpus.

\section{ASPECTS TYPOLOGIQUeS}

À peine un quart des individus présente une morphologie restituable, le reste étant trop fragmenté pour être déterminé (fig. 12). La série est largement dominée par des formes simples $(83 \%)$, des vases à profil en $« \mathrm{~S} »(8,5 \%)$ et des bouteilles $(8,5 \%)$. Pour les formes simples, les récipients sont en moyenne de petit gabarit (bol, coupe...) avec un diamètre à l'embouchure souvent plus large que la hauteur (tabl. 3). Les panses sont aussi plus larges que les ouvertures avec des bords légèrement rentrants.

Les cols sont peu développés sauf pour la catégorie des bouteilles et un vase à col divergeant mais dans l'ensemble l'obliquité des cols est faible et le resserrement entre le col et l'embouchure est peu marqué.

Cette apparente uniformité dans ces productions cache quelques nuances au sein des formes simples et complexes. Les profils en $1 / 2$ ou $3 / 4$ de sphère ont le plus souvent des parois droites, parfois légèrement rentrantes et rarement divergentes (fig. 13,
Tabl. 3 - Morphologie des vases complets ou conservés sur une moitié de profil (I. Praud, Inrap).

$\boldsymbol{H t V}$ : hauteur du vase; $\mathbf{D e}$ : diamètre à l'embouchure; $\boldsymbol{D p}$ : diamètre maximum de la panse ; HtC : hauteur du col ; HtP : hauteur de la panse ; $\boldsymbol{D c}$ : diamètre du col.

Morphology of the whole pottery vessels or vessels with half the profile intact (I. Praud, Inrap).

$H t V$ : height of vessel; De: diameter at the mouth; Dp: maximum diameter of the body; HtC: height of the neck; HtP: height of the body; Dc: diameter of the neck.

\begin{tabular}{|c|c|c|c|c|c|c|c|}
\hline $\mathbf{N}^{\circ}$ inventaire & Sites & $\mathrm{HtV}$ & De & Dp & $\mathrm{HtC}$ & $\mathrm{HtP}$ & Dc \\
\hline 1 & LSL1 & 9,8 & 13 & 14,4 & & & \\
\hline 2 & LSL 2 & 17,5 & 15,5 & 22,9 & & & \\
\hline 3 & LSL 3 & & 22,8 & 24,2 & & & \\
\hline 4 & LSL 6 & 8,7 & 12,9 & & & & \\
\hline 5 & LSL 7 & 9,9 & 13 & 13,9 & & & \\
\hline 6 & LSL 8 & & 41 & 42,2 & & & \\
\hline 7 & LSL 9 & 7,2 & 3,1 & 7,6 & 1,8 & 5,4 & \\
\hline 8 & LSL 10 & & 31,3 & 30 & & & \\
\hline 9 & LSL 11 & 10,2 & 16,9 & 17,5 & & & \\
\hline 10 & LSL 12 & 21,7 & 18,9 & 28,3 & & & \\
\hline 11 & LSL 13 & 11,5 & 21,6 & 21,3 & & & \\
\hline 12 & LQ 14 & 19,4 & 20,2 & 21,9 & & & \\
\hline 13 & LQ 15 & 11 & 13,2 & 14,2 & & & \\
\hline 14 & LQ 16 & 27 & 23,5 & 27,3 & 4,6 & & 20 \\
\hline 15 & LQ 17 & & 17,2 & & & & \\
\hline 16 & LQ 18 & & 15,8 & 19,3 & & & \\
\hline 17 & LQ 19 & & 17,4 & 19,5 & & & \\
\hline 18 & LQ 20 & 12 & 11,2 & 13,5 & 1,1 & 10,9 & 11,1 \\
\hline 19 & LQ 21 & 23,5 & 31,4 & 31,7 & & & \\
\hline 20 & LQ 22 & 16,6 & 20,3 & 22 & & & \\
\hline 21 & LQ 23 & 15,3 & 20,7 & 21,2 & & & \\
\hline 22 & Vitry 24 & 8,8 & 10,1 & 12,7 & & & \\
\hline 23 & Vitry 25 & 12,7 & 15,9 & 17,8 & & & \\
\hline 24 & Vitry 26 & & 44,1 & 53,1 & & & \\
\hline 25 & Vitry 27 & 33,2 & 39,8 & 38,2 & 5 & 27,8 & 37 \\
\hline 26 & Vermand 28 & 10,9 & 13,7 & 15,6 & & & \\
\hline 27 & Vermand 29 & 4,5 & 19,8 & 19,6 & & & \\
\hline 28 & Vermand 30 & & 26,8 & 26,5 & & & \\
\hline 29 & Vermand 31 & & 12,2 & 18,8 & & & 8,3 \\
\hline 30 & Étouvie 32 & 14 & 19,7 & 20,2 & & & \\
\hline 31 & Étouvie 33 & 17 & 13,7 & 19,8 & & & \\
\hline \multirow[t]{2}{*}{32} & Étouvie 34 & & 28,2 & 31,6 & & & \\
\hline & $\begin{array}{l}\text { moyenne } \\
\text { écart-type }\end{array}$ & $\begin{array}{c}14,65 \\
5,39\end{array}$ & $\begin{array}{c}20,15 \\
6,86\end{array}$ & $\begin{array}{c}23,02 \\
7,10\end{array}$ & $\begin{array}{l}3,13 \\
1,68\end{array}$ & \begin{tabular}{|c|}
14,70 \\
8,73
\end{tabular} & $\begin{array}{l}19,10 \\
9,40\end{array}$ \\
\hline
\end{tabular}

$\mathrm{n}^{\circ} 2-4,6-7,18 \ldots$. . Les gabarits sont variés dans cette catégorie, du vase de présentation souvent munis d'un organe de préhension à des récipients de grande contenance à profil en « $U$ »" (fig. 13, $\mathrm{n}^{\circ} 18$ ). Les individus à col plus ou moins bien dégagé sont peu nombreux (fig. 13, no 19-22 et 24). Seules les bouteilles (aucune n'est complète) et un vase de Languevoisin-Quiquery présentent des cols nettement dégagés (fig. 13, $\mathrm{n}^{\circ}$ 21-22). Une micro-bouteille présente un col court (fig. 13, $\mathrm{n}^{\circ} 24$ ). Les bords ont une légère divergence ou bien présentent de petits cols droits naissants à la jonction avec la panse (fig. 13, no 26-27). 
Fig. 10 - Répartition des différents types de dégraissant utilisés pour la fabrication des récipients en terre cuite $(\boldsymbol{A})$ et par site (B ; I. Praud, Inrap).

LSL : Loison-sous-Lens (62),

VCB : Vitry-en-Artois (62),

$V C L:$ Vermand (02),

LQ : Languevoisin-Quiquery (80),

ECM : Amiens «Étouvie » (80).

Distribution of the different types of pottery temper manufacturing processes $(A)$ and by settlement (B; I. Praud, Inrap).

LSL: Loison-sous-Lens (62),

$V C B$ : Vitry-en-Artois (62),

VCL: Vermand (02)

LQ: Languevoisin-Quiquery (80),

ECM: Amiens "Étouvie" (80).

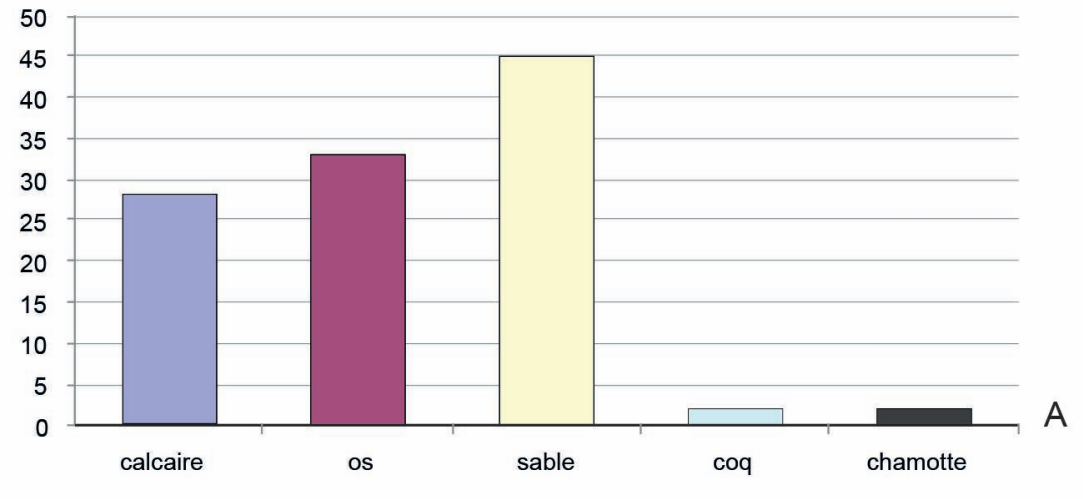

dégraissants ajoutés $(\mathrm{nr}=110)$

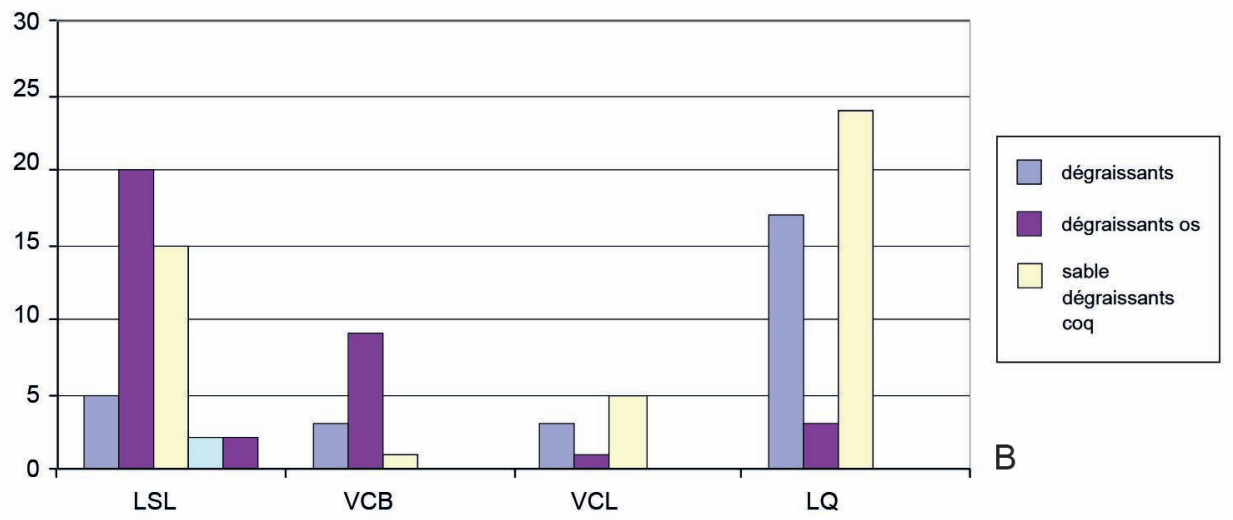

sites
Fig. 11 - Répartition des couleurs de cuisson (A) et par site (B; I. Praud, Inrap).

$\boldsymbol{C}$ : claire ; $\boldsymbol{F}$ : foncée ; Interne / Cour / Externe LSL : Loison-sous-Lens (62),

VCB : Vitry-en-Artois (62), VCL : Vermand (02), LQ : Languevoisin-Quiquery (80),

ECM : Amiens «Étouvie » (80).

Pottery firing manufacturing processes $(A)$ and by settlement ( $B$; I. Praud, Inrap).

C: light colour; F: dark; Internal/Centre/External

LSL: Loison-sous-Lens (62),

$V C B$ : Vitry-en-Artois (62),

VCL: Vermand (02),

LQ: Languevoisin-Quiquery (80),

ECM: Amiens "Étouvie" (80).

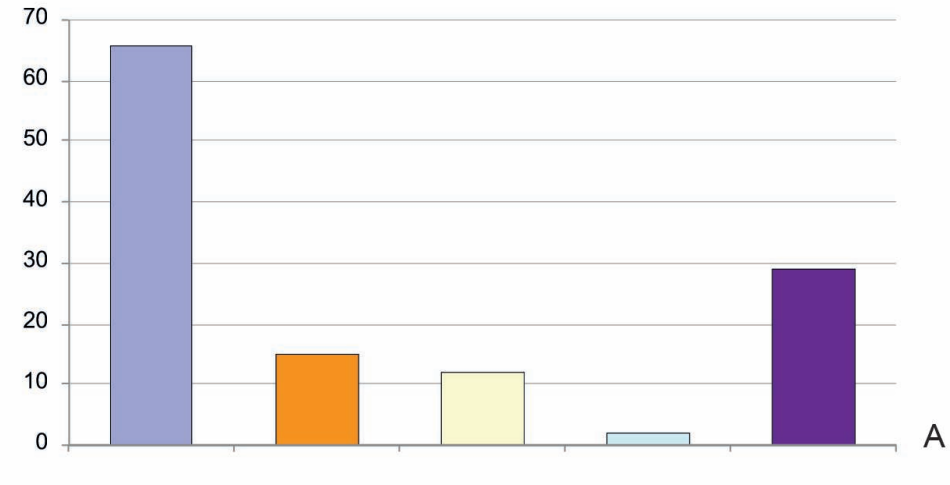

$\begin{array}{lllll}\text { CFC } & \text { CCC } & \text { FFF } & \text { FFC }\end{array}$

Couleur de cuisson des vases $(n r=124)$

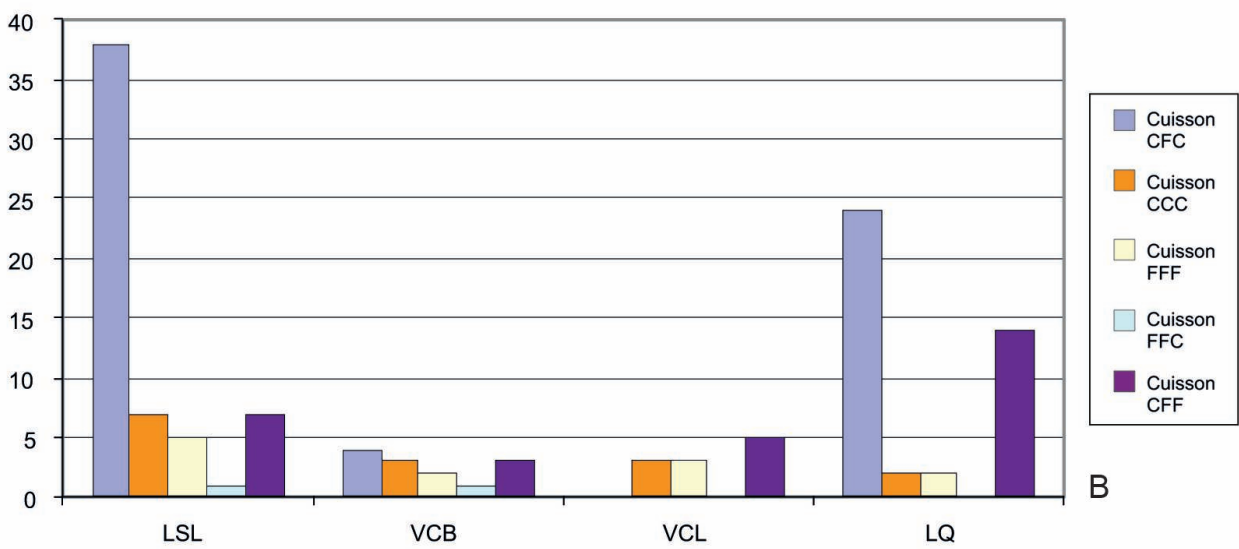




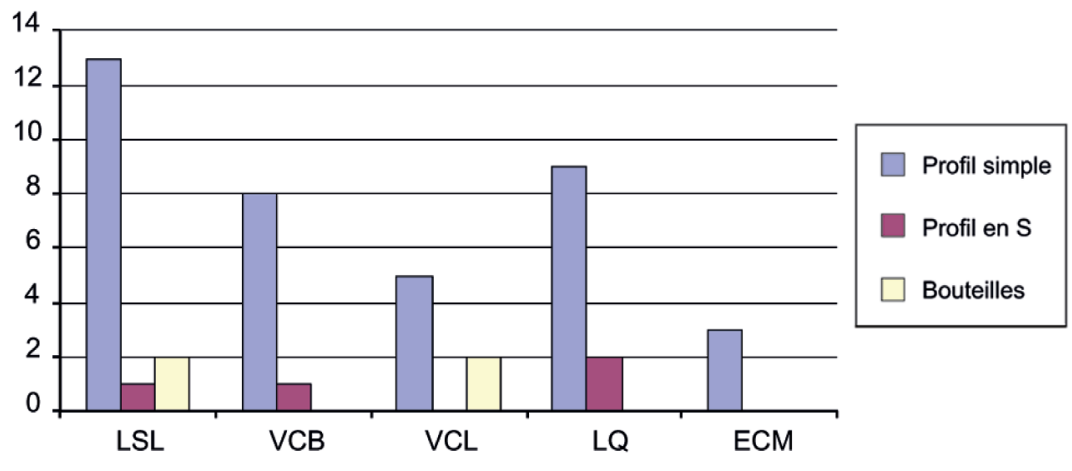

Profils simples

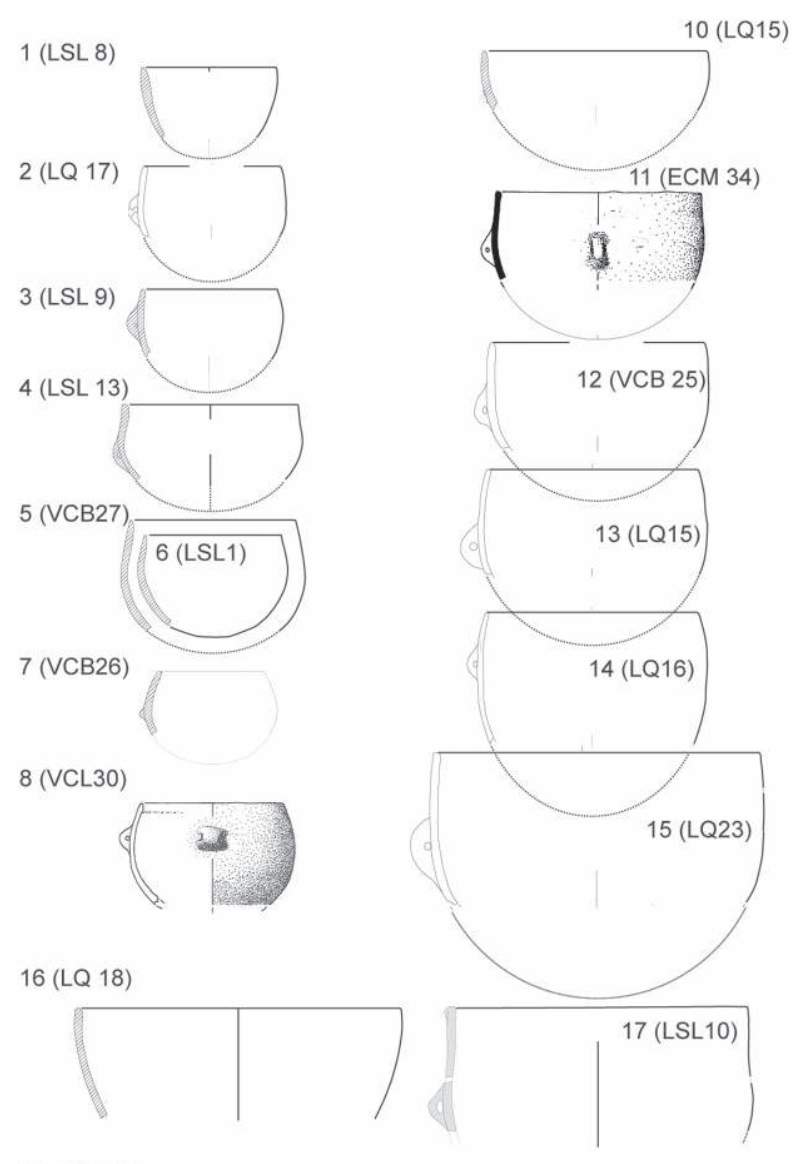

18 (VCL28)
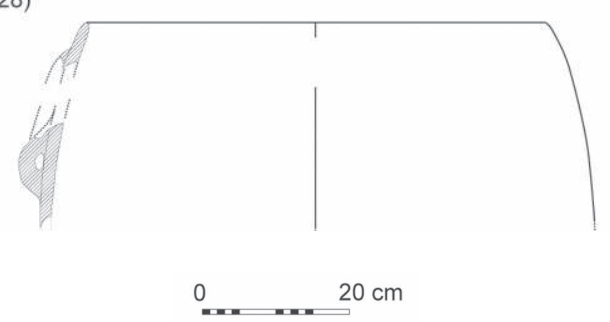

Fig. 12 - Répartition des formes par site (I. Praud, Inrap).

LSL : Loison-sous-Lens (62), VCB : Vitry-enArtois (62), VCL : Vermand (02), LQ: LanguevoisinQuiquery (80), ECM : Amiens "Étouvie» (80).

Distribution of pottery shapes by site (I. Praud, Inrap).

LSL: Loison-sous-Lens (62), VCB: Vitry-enArtois (62), VCL: Vermand (02), LQ: LanguevoisinQuiquery (80), ECM: Amiens "Étouvie" (80).
Profils complexes

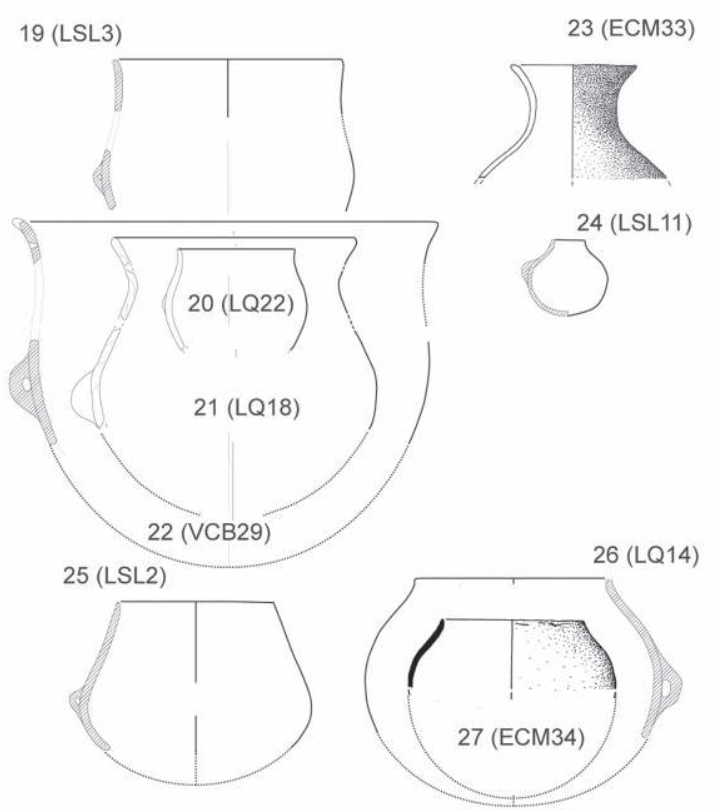

Fig. 13 - Les profils simples et complexes des vases du Néolithique ancien du Nord-Ouest de la France (Collectif, Inrap). Les numéros des vases entre parenthèse permettent de se reporter au numéro d'inventaire du tableau 3 pour les dimensions.

LSL : Loison-sous-Lens (62), VCB : Vitry-en-Artois (62), VCL : Vermand (02), LQ : Languevoisin-Quiquery (80), ECM : Amiens «Étouvie » (80).

Simple and complex profiles of Early Neolithic pottery from northwestern France (Collectif, Inrap). The numbers in brackets refer to the inventory numbers in table 3 to identify the provenance of the pottery.

LSL: Loison-sous-Lens (62), VCB: Vitry-en-Artois (62), $V C L$ : Vermand (02), LQ: Languevoisin-Quiquery (80), ECM: Amiens "Étouvie" (80). 
La morphologie des vases correspond aux standards de la culture BVSG, la répartition des formes selon les sites ne montre pas de tendances évolutives marquées. La présence d'une micro-bouteille est rare en contexte BVSG, mais elles sont cependant attestées : un exemplaire, à col plus développé, existe à Mareuil-les-Meaux "les Vignolles » (Lanchon et al. 2006, fig. 8, p. 303), d'autres à Pontpoint « le Fond de Rambourg " (Oise ; Bostyn et al. 2012). La fonction des micro-vases reste aujourd'hui indéterminée.

\section{LES VASES DÉCORÉS}

La faiblesse des effectifs décorés interdit toute comparaison en pourcentage des différentes catégories de décor (tabl. 4). L'étude des plans de site - et la recherche de remontages interfosses - n'a pas permis la restitution fiable d'unités d'habitation danubiennes. Tout cumul d'effectifs de fosses différentes serait trop aléatoire. Les commentaires qui suivent ont donc une valeur essentiellement typologique mais serviront néanmoins à un essai de périodisation des différents gisements.

Les décors incisés en arêtes de poisson sont typiques de la culture BVSG, mais sont très peu représentés ici. En effet, seul le site de Loison-sous-Lens livre un vase fortement dégraissé à l'os pilé et brûlé (ST 1008 ; fig. 14, nº 1), et décoré d'incisions profondes, larges et très courtes, constituant un large bandeau sous le bord et sur le haut du vase ainsi que des panneaux quadrangulaires sur la panse. La forme et la technique du décor sont tout à fait comparables à un vase quasi-identique (hormis le fond conique) provenant du site d'Irchonwelz « la Bonne Fortune » (Constantin et al. 1978, fig. $6, \mathrm{n}^{\circ} 28$ ) et d'un second très proche provenant de Vaux-etBorset « Gibour » (Caspar et al. 1993, pl. 41, n 18).

Les décors imprimés au peigne sont peu nombreux et proviennent surtout du site de Loison-sous-Lens. Un récipient à bords rentrants et doté de trois larges préhensions à perforation horizontale, présente un décor composé de guirlandes suspendues à une bande horizontale accompagné sur la panse de bandes rectangulaires imprimées (ST 1008, fig. 14, $\mathrm{n}^{\circ} 2$ ). Le décor est réalisé par impressions pivotantes serrées d'un peigne très fin à quatre dents et à front légèrement curviligne. Le décor sous le rebord est constitué d'une bande horizontale à laquelle sont suspendues une série de guirlandes elles aussi imprimées ; le décor principal, couvrant, est constitué de nombreux segments de courtes bandes verticales, couvrant la totalité de la panse. Un vase à profil simple est représenté par une vingtaine de fragments, il porte un décor assez couvrant, combinant incisions et impressions pivotantes d'un peigne à 7 dents (ST 11, fig. 14, n 3). Le décor est constitué d'une ligne de triangles incisés remplis d'impressions au peigne sous le rebord et de panneaux larges bordés de lignes incisées verticales, remplis de bandes d'impressions sur la panse et séparés par d'étroites bandes vides. La hauteur des triangles sous le rebord varie de façon assez importante, mais il ne peut s'agir de deux vases différents. Deux tessons découverts à Étouvie (fig. 14, no 4-5) présentent aussi un décor au peigne : dans le premier cas il s'agit d'impressions pivotantes au peigne

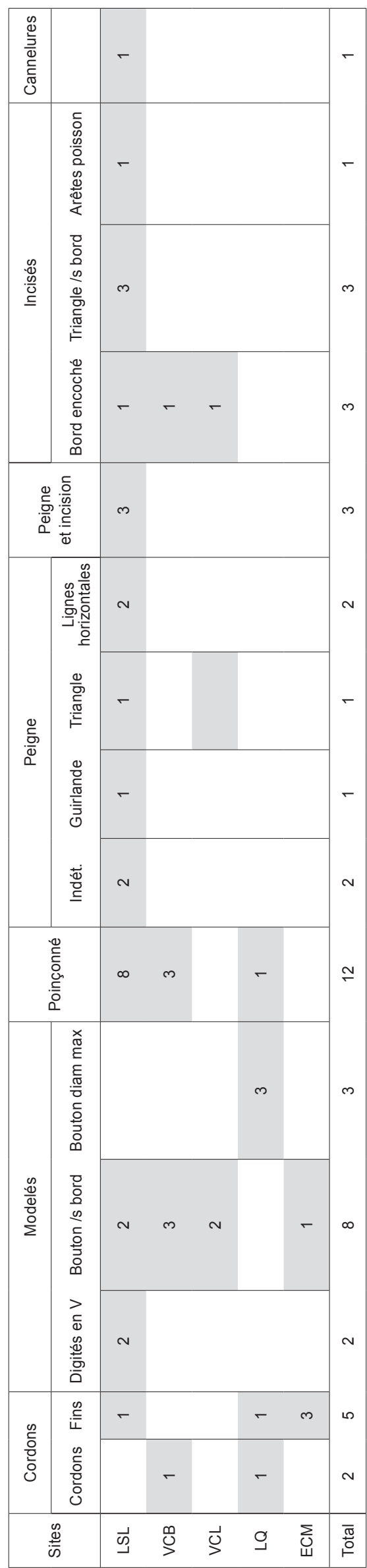




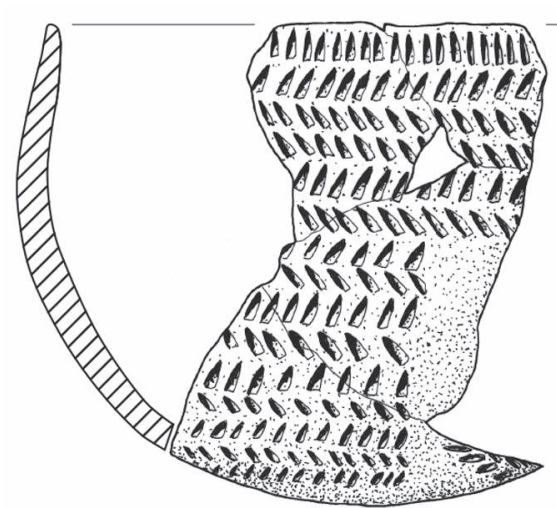

$1(1)$

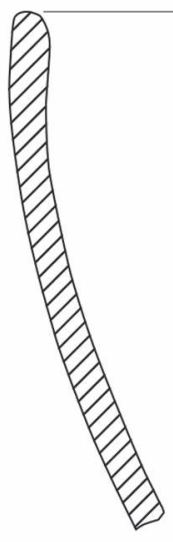

$3(10)$
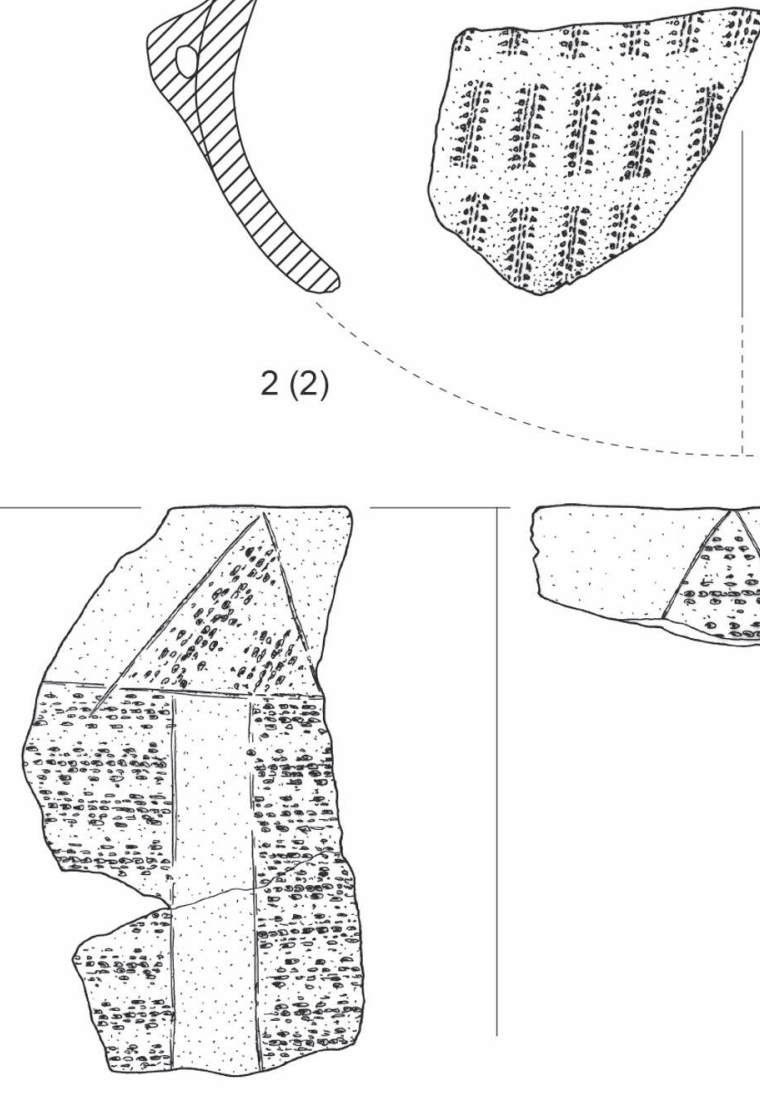

$2(2)$
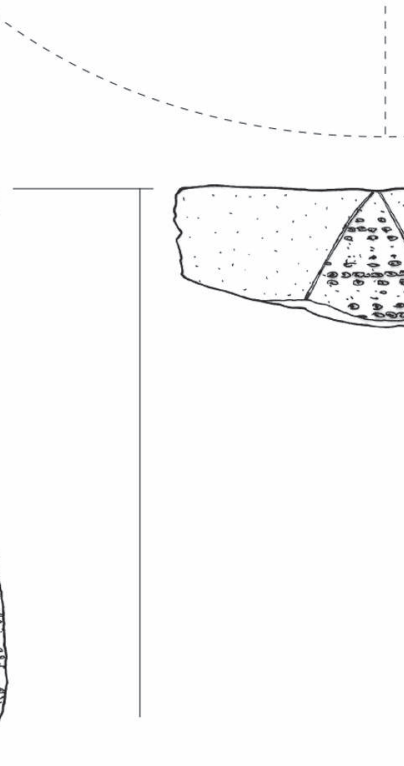

$10 \mathrm{~cm}$
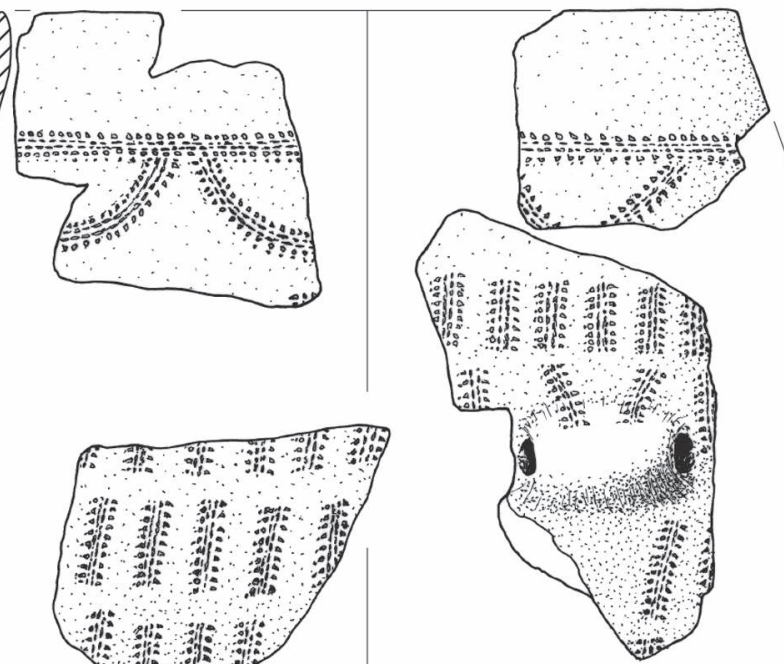
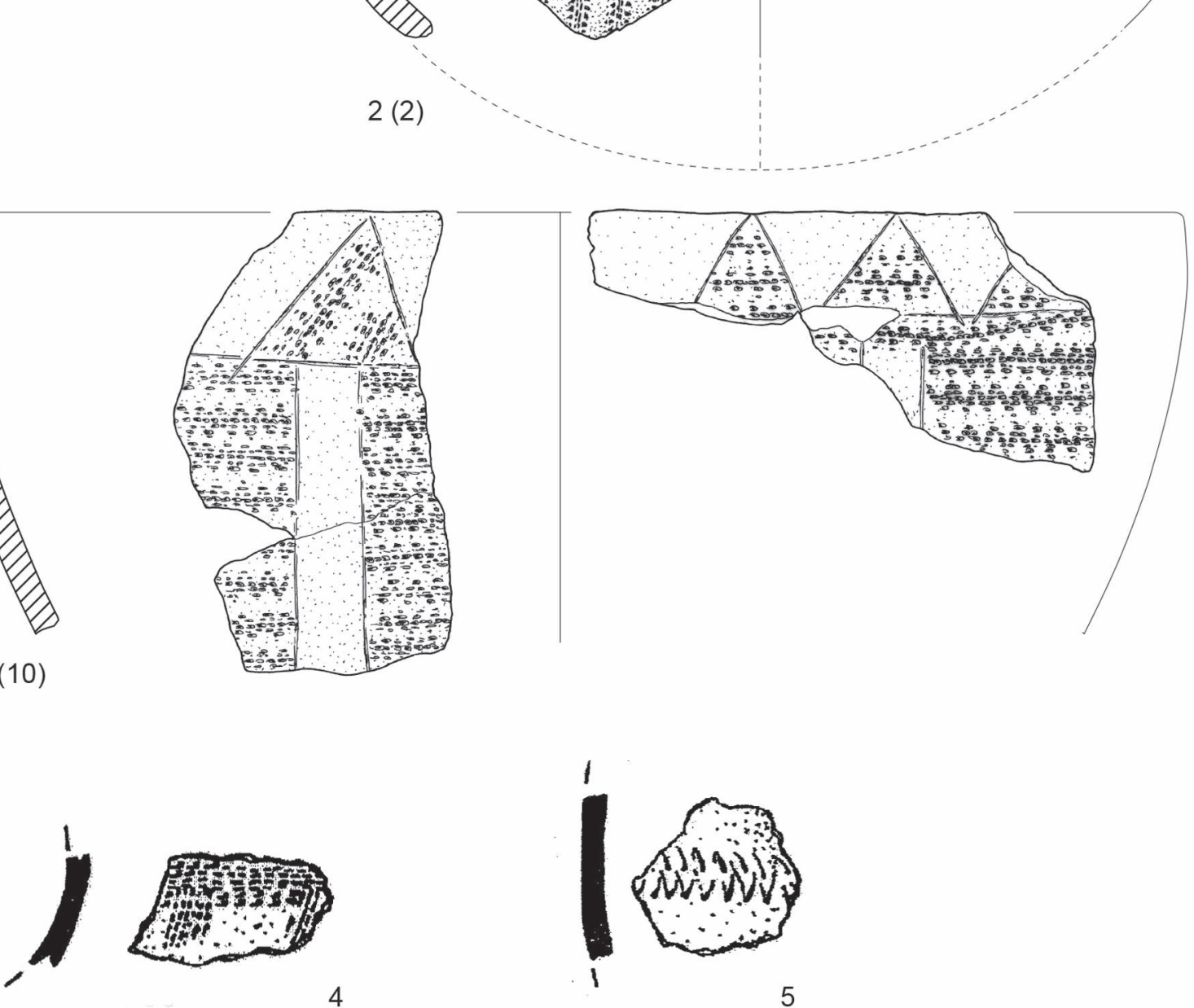

Fig. 14 - Les décors imprimés au peigne (dessins 1-3: Y. Lanchon, Inrap; dessins 4-5 : F. Prodéo, Inrap). Comb-printed decoration on pottery (drawings 1-3: Y. Lanchon, Inrap; drawings 4-5: F. Prodéo, Inrap). 
à trois dents mais le motif (guirlande ou en « $\mathrm{T} »)$ reste délicat à restituer ; quant au second, seules des empreintes d'impressions pivotantes sont conservées.

Les motifs de décor couvrant au peigne sont caractéristiques du BVSG, même si les métopes imprimées horizontalement sont plus courantes, à Vaux-et-Borset « Gibour » (Caspar et al. 1993, pl. 19, no 15 et pl. 30, n 30 ) ou dans le BVSG, à Poses « Sur la Mare » (Bostyn et al. 2003a, fig. 84, n 11). Quant aux guirlandes suspendues à une bande horizontale, elles existent sur pratiquement tous les sites BVSG livrant des céramiques décorées au peigne. L'organisation de triangles remplis au peigne sous le rebord / panneaux couvrants imprimés au peigne sur la panse est attestée, tant dans le Blicquy, à Vaux-et-Borset « Gibour» (Constantin et al. 1993, pl. 53 n $^{\circ} 19$ et pl. 54, no 17) et à Irchonwelz (ST 21 ; inédit) que dans le BVSG, à LongueilSainte-Marie « la Butte de Rhuys » (Bostyn et al. 1993).

Les décors poinçonnés employés seuls ou associés à des incisions sont les plus représentés sur les sites de Loison-sousLens et de Vitry-en-Artois.

Le thème des triangles incisés sous le bord est représenté sur un vase à profil en « $\mathrm{S}$ ». Il possède un décor couvrant qui combine incisions et impressions d'un poinçon à extrémité arrondie (ST 1008, fig. 15, n ${ }^{\circ} 1$ ). Il est constitué d'une ligne de triangles incisés sous le rebord, pointe en haut et remplis d'impressions, puis d'une succession de panneaux ou de segments de lignes horizontales poinçonnées et d'au moins une bande interrompue de triangles à remplissage poinçonné. Ce vase est doté de préhensions (deux conservées) à perforation funiculaire horizontale rapportées très probablement en symétrie ternaire au diamètre maximum de la panse. Deux autres tessons de rebord en céramique fine appartenant à un vase à profil en « $S$ » et au col peu marqué portent aussi un riche décor. Le rebord est encoché de très courtes incisions et décoré d'une rangée de triangles incisés, pointes en haut à remplissage croisillonné ; le haut de la panse développe un décor poinçonné apparemment couvrant, constitué de lignes horizontales et régulières de coups de poinçon fin (ST 107, fig. 15, $\mathrm{n}^{\circ}$ 2). Associés dans la même fosse, quatre tessons très fragmentés en céramique portent un décor partiellement reconstituable d'une rangée horizontale de triangles incisés à remplissage poinçonné, probablement située sous le rebord.

L'utilisation d'un second type de poinçon laisse des impressions plus grosses et plus profondes avec une forme allongée. On le trouve notamment sur un fragment de rebord légèrement ouvert et décoré d'une bande de triangles (pointe dirigée vers le bas) à remplissage poinçonné ; les triangles, non adjacents, sont disposés juste sous le rebord et ne sont pas délimités par une ligne incisée (ST 1137, fig. 15, no 3).

Plusieurs fragments de petite taille présentent des décors poinçonnés dont l'organisation est difficile à restituer. Il s'agit notamment de tessons de Loison-sous-Lens portant une ligne horizontale de grosses impressions au poinçon sous le bord (fig. 15, $\mathrm{n}^{\circ} 4$ ) et d'une préhension à décor poinçonné de grosses impressions (fig. 15, $\mathrm{n}^{\circ}$ 5). À Vitry-en-Artois, trois tessons sont décorés : de lignes poinçonnées probablement horizontales (fig. 15, no 6-7), de deux lignes de coups de poinçon en forme de virgule et d'un bord à lèvre aplatie décoré de bandes de ligne poinçonnée oblique (fig. 15, $\mathrm{n}^{\circ} 8$ ).

Le thème des triangles sous le bord est très fréquent, tant dans le Blicquy que le VSG, surtout dans leur variante " pointe en haut ", moins fréquemment " pointe en bas " (Poses, M6, fig. $80 \mathrm{n}^{\circ}$ 5). Le remplissage des triangles le plus fréquent est celui des hachures obliques (Poses, M40, fig. 84, $\mathrm{n}^{\circ} 12$; Poses, M50, fig. 86, $\left.\mathrm{n}^{\circ} 4\right)$, plus rarement « croisillonnées », comme à Irchonwelz (Constantin 1985, p. 117, n 7-3), Marcilly (Constantin 1985, fig. 197, n 18), Vignely « la Porte aux Bergers » (Bostyn et al. 2018). Le remplissage au peigne est également attesté (Poses, M80, fig. 96, n 11). Le remplissage poinçonné est plus rare mais attesté à Lery (Bostyn et al. 2003a, fig. 110, $\mathrm{n}^{\circ}$ 11) et à Rungis « les Antes » (Bostyn et al. 2002, fig. 58, $\mathrm{n}^{\circ} 2$ ).

Les tessons à décor poinçonné évoquent les décors attestés dans la culture de Cerny et plus particulièrement son faciès éponyme (Constantin 1985, Louboutin et Simonin 1997), mais le caractère réduit des tessons interdit de discuter sur les thématiques. Rappelons, cependant, que ce type de décor est aussi attesté dans la culture BVSG. Elle semble augmenter dans l'étape récente de cette culture (Lanchon 2008). Les panneaux poinçonnés couvrants sont attestés dans le Blicquy, à Irchonwelz (Constantin 1985, fig. 11.1, 12.4, et 12.6).

Les décors en "V 》 sont illustrés notamment sur une coupe hémisphérique décorée d'une ligne d'impressions ongulées sous le rebord et d'une ligne oblique réalisée selon la même technique sur le haut de la panse. Un épaississement de la pâte indique que la ligne oblique joignait le rebord à un moyen de préhension disparu. Il s'agit bien évidemment d'une organisation du décor en «V » (ST 1074, fig. 15, $n^{\circ}$ 9). Toujours à Loison-sous-Lens, un grand vase à provisions à bords droits (ST 1049, fig. 16, n 1 ) porte un décor constitué de pincements en « V » partant de l'organe de préhension pour rejoindre une ligne horizontale de boutons rapportés sous le bord. Ces deux décors en « V » attestés uniquement à Loison-sous-Lens (ST 1074 et 1049) trouvent de très nombreuses comparaisons dans le Bassin parisien, sur pratiquement tous les sites et en particulier à Misy/Yonne « le Bois des Refuges » (Mordant et al. 1977, fig. 3) et Poses (Bostyn et al. 2003a, fig. 82, $\mathrm{n}^{\circ} 1$ et fig. 103, $\mathrm{n}^{\circ} 1$ ), où on retrouve la même association ligne de boutons sous le rebord « V » ongulé ou pincé. Rappelons que les décors en «V》ne sont pas attestés à Irchonwelz « la Bonne Fortune »; cette absence avait été interprétée comme un marqueur chronologique (Constantin 1985).

Trois tessons de rebord appartiennent à un récipient à bords droits et à pâte fine, ils sont ornés, à $1 \mathrm{~cm}$ sous le rebord, d'un cordon horizontal lisse et peu épais, qui ne semble pas avoir été rapporté, mais plutôt façonné dans la masse. Un vase de grande dimension est décoré à Vitry-en-Artois d'un cordon lisse en « V » dont seule la partie accrochée à l'organe de préhension est conservée (fig. 16, n 2). À LanguevoisinQuiquery, un vase de grande dimension à bord droit et lèvre aplatie présente un cordon lisse peu marqué, s'étirant de la préhension vers le rebord en «V » (fig. 16, n 3). À Étouvie, deux tessons présentent des décors plastiques : un grand vase 

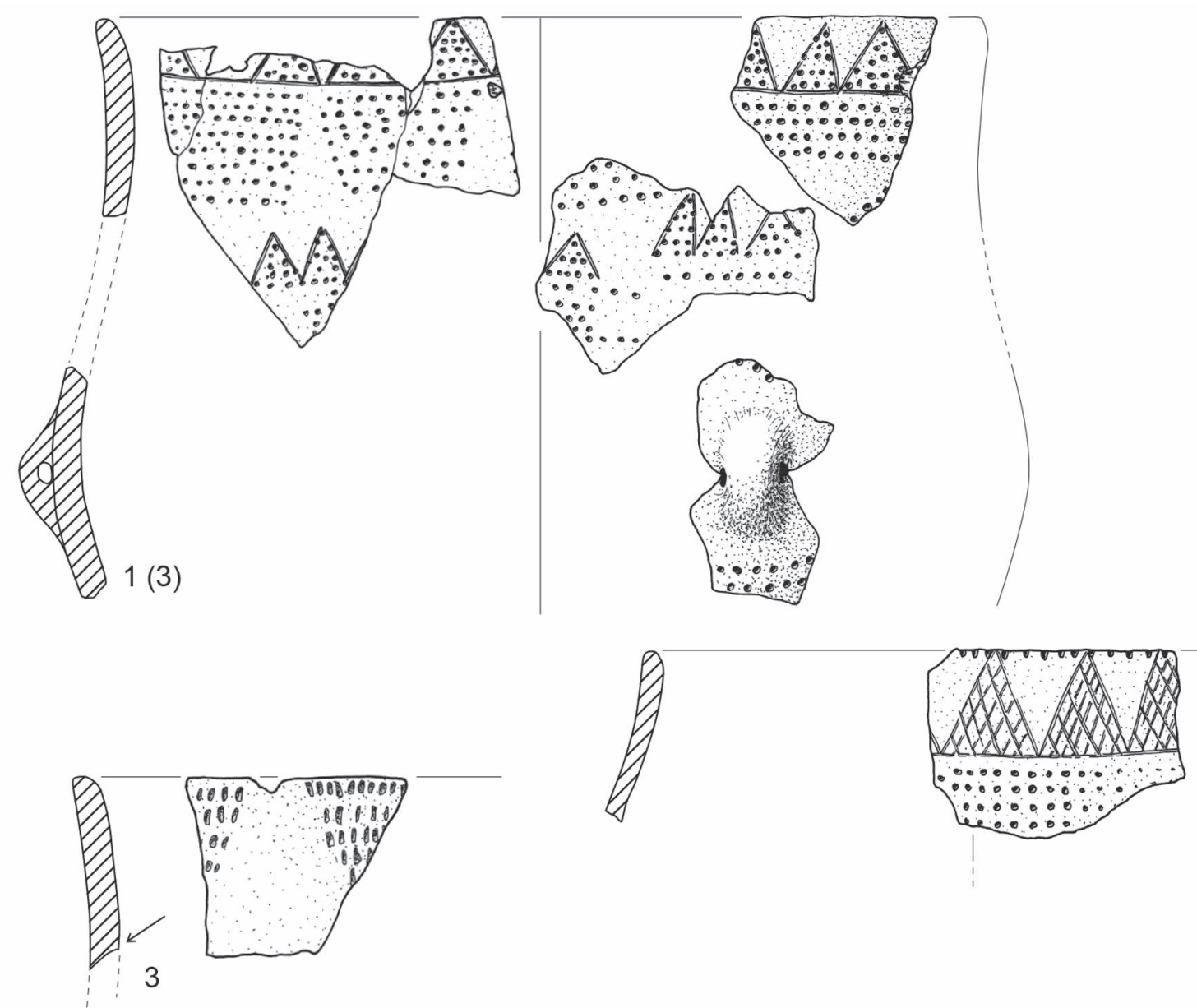

\section{2}

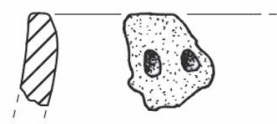

4

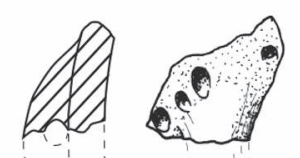

5

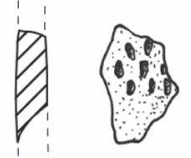

6

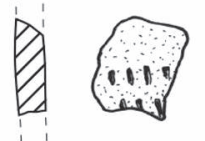

7

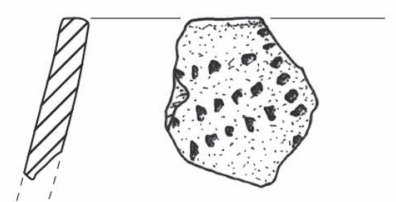

8

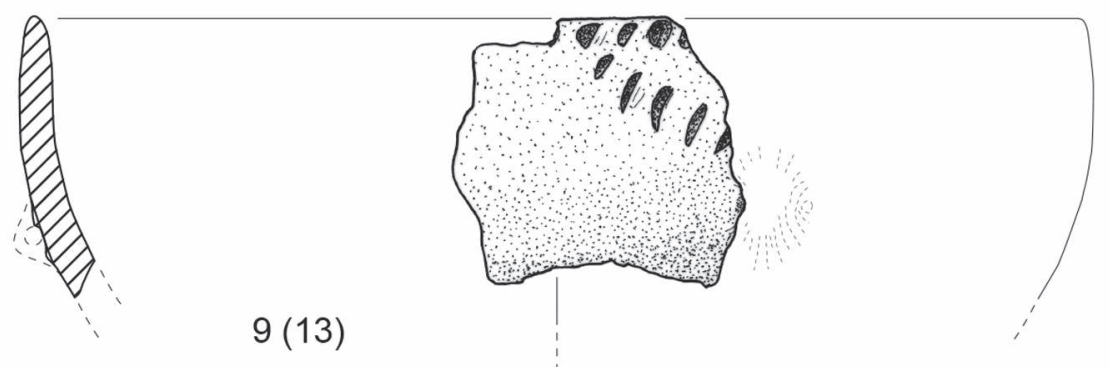

$10 \mathrm{~cm}$

Fig. 15 - Les décors poinçonnés (dessins Y. Lanchon, Inrap).

Stamp decoration on pottery (drawings $Y$. Lanchon, Inrap). 
Fig. 16 - Décors de pincements en « $V$ » (dessins 1-2: Y. Lanchon, Inrap; dessin $3: \mathrm{N}$. Vandamme, Inrap).

"V"-shaped pinched decoration (drawings 1-2: Y. Lanchon; drawing 3: N. Vandamme, Inrap).

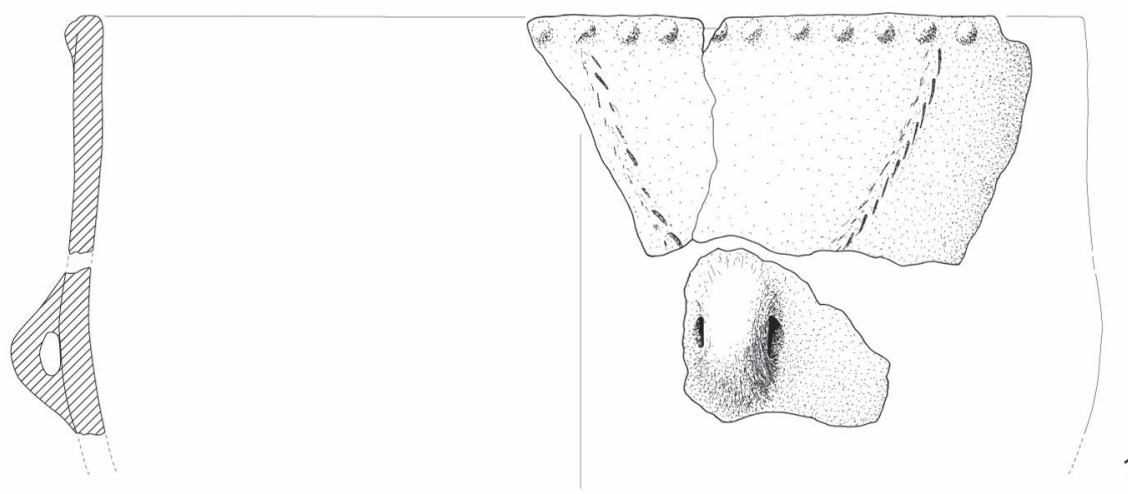

1
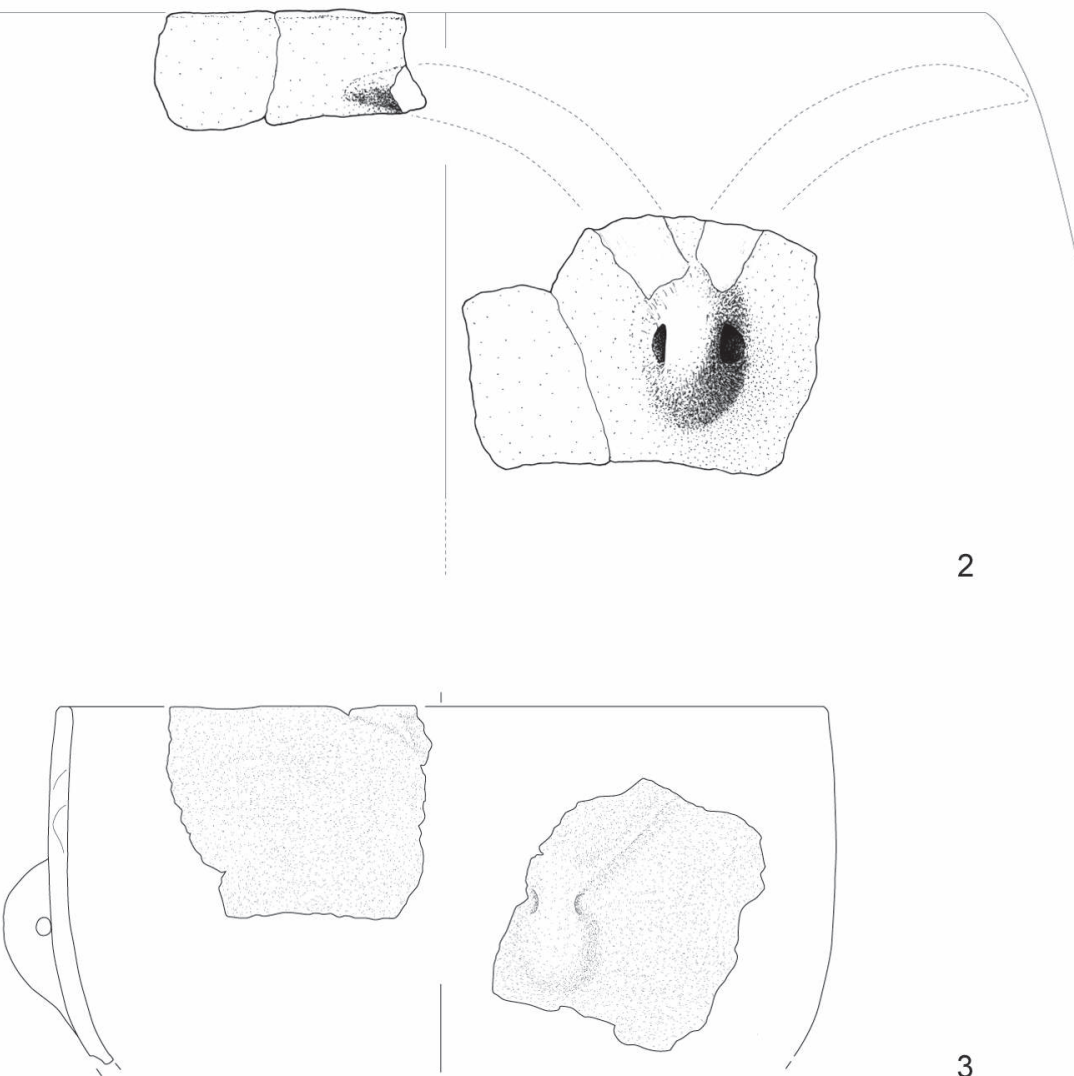

3
0

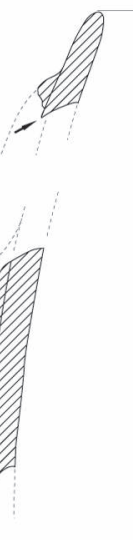

à décor de petits boutons proéminents sur le bord associés à des gros boutons allongés verticaux (préhension ?) dont partent des cordons en direction du bord (en «V»?). À cela s'ajoutent deux tessons avec un cordon horizontal sous le bord.

Plusieurs exemplaires à Vitry-en-Artois portent des mamelons jumelés sur un vase de grandes dimensions (fig. 17, $\mathrm{n}^{\circ}$ 1), ainsi qu'un grand vase à rebord orné de boutons dépassant le bord (fig. 17, $\mathrm{n}^{\circ}$ 2). À Languevoisin-Quiquery, deux fragments de récipient portent au-dessus du diamètre maximum de la panse deux boutons séparés (fig. 17, $\mathrm{n}^{\circ}$ 3-4). Signalons enfin, trois récipients à Étouvie munis de boutons jumelés sous la lèvre et de boutons seuls sur une bouteille et un vase de grandes dimensions à Vermand.
Les décors plastiques de cordons lisses et les boutons doubles sont traditionnellement considérés comme caractéristiques d'une étape récente, voire finale, tant du VSG que du Blicquy (Constantin 1985, Lanchon 1984 et 2008). Il faut préciser que les cordons en question sont rapportés, la plupart du temps très saillants, et utilisés dans la réalisation d'un motif en « V » tendu entre anse et rebord, ainsi que les motifs dérivés. Si la plupart des récipients peuvent être classés dans cette catégorie, en revanche le petit cordon horizontal sous le bord issu de la structure 11 de Loison-sous-Lens et ceux venant d'Étouvie n'appartiennent pas à cette catégorie, et il est probable que ces petits cordons, qui existent sur céramique fine, associés ou non à d'autres techniques décoratives (par 


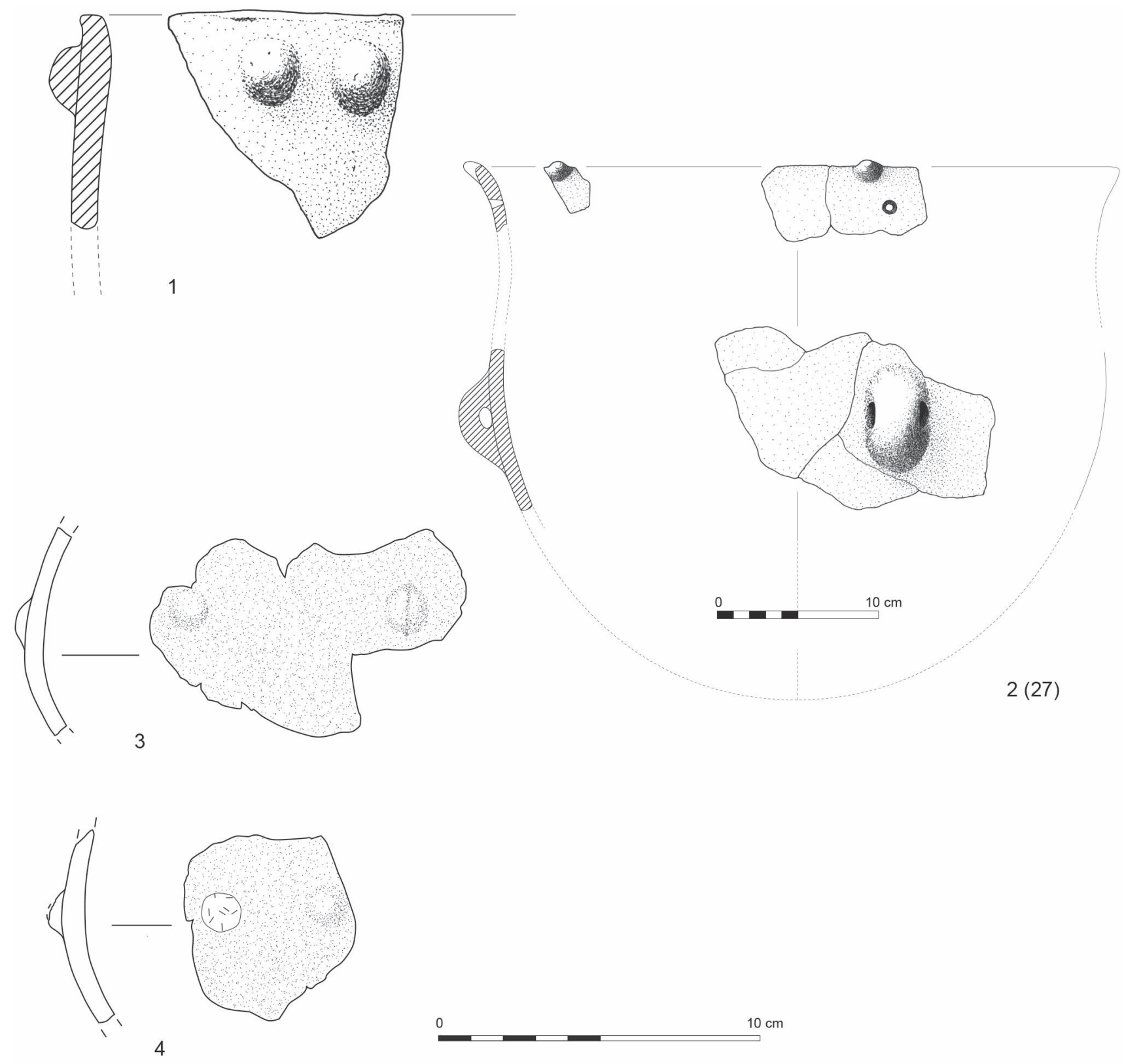

Fig. 17 - Décors de mamelons (dessins 1-2 : Y. Lanchon, Inrap; dessins 3-4 : N. Vandamme, Inrap).

Nipple-shaped decoration on pottery (drawings 1-2: Y. Lanchon; drawings 3-4: N. Vandamme, Inrap).

exemple à Vignely « la Porte aux Bergers », M90 et M150) apparaissent ponctuellement dès l'étape ancienne de la culture BVSG comme dans le Rubané final du bassin de la Seine (Meunier 2012). Enfin, les boutons dépassant le rebord sont parfois présents dans le Cerny (Bailloud 1964, Constantin 1985) mais il est plus que probable que cet élément décoratif caractérise l'étape finale de la culture BVSG. Ils sont notamment attestés en contexte BVSG à Échilleuses (Simonin 1997) ou à Poses « le Vivier» (Billard et al. 1994, Lanchon in Bostyn et al. 2003a).

En résumé, les ensembles céramiques identifiés sur ces gisements sont attribuables sans aucun problème à la culture de Blicquy-Villeneuve-Saint-Germain. Les comparaisons typo- logiques (techniques décoratives) et surtout technologiques (dégraissant à l'os) renvoient pour deux gisements, Loisonsous-Lens et Vitry-en-Artois, plutôt au faciès régional Blicquy de la vaste culture du BVSG, bien que, on l'a vu, les comparaisons ne soient pas exclusives.

Quant à la place chronologique de ces ensembles, il est difficile de s'étendre compte tenu de la faiblesse des effectifs. Toutefois, l'existence (mais pas dans les mêmes fosses) d'incisions en « arêtes de poisson » et de « V » modelés, et l'absence de véritables cordons inciteraient à placer le site de Loisonsous-Lens dans une étape moyenne de la culture BVSG tandis que le reste des sites s'intégrerait dans les étapes récente et finale de cette culture. 


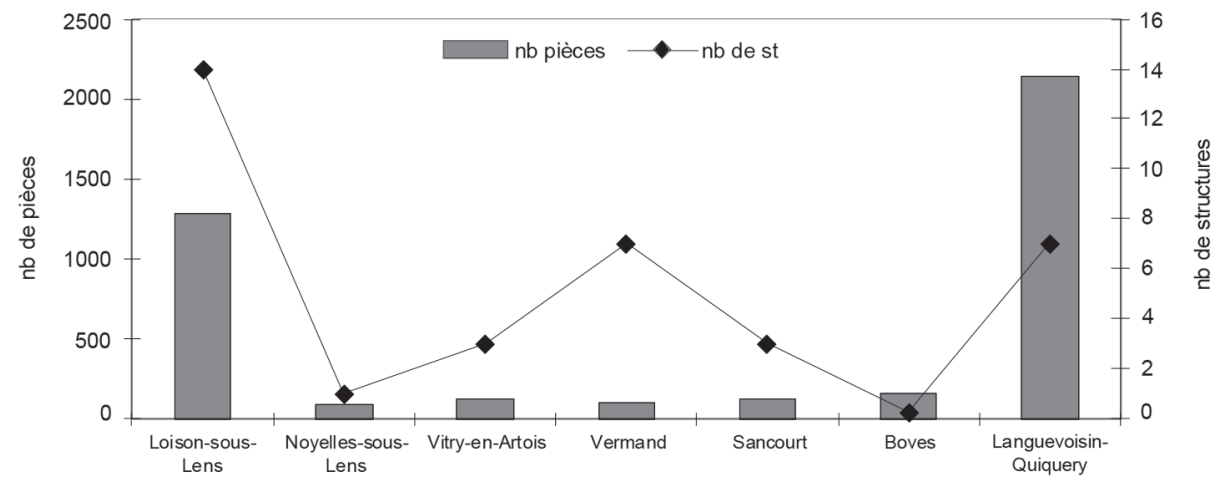

Fig. 18 - Quantité d'artefacts en silex sur chaque site étudié et nombre de structures dont il est issu (F. Bostyn, Inrap).

Number of flint artefacts from each studied site and number of structures from which they derive (F. Bostyn, Inrap).

\section{APPROCHE TECHNO-FONCTIONNELLE DU MOBILIER LITHIQUE}

\section{Présentation générale}

Même si le silex fait partie des matériaux qui se conservent parfaitement bien et est, de fait, souvent l'un des rares matériaux disponibles, les conditions d'acquisitions des données exposées précédemment ont néanmoins un impact direct sur les assemblages étudiés. La valeur informative n'est de fait pas la même et les degrés d'analyse sont inégaux entre les séries provenant de sites fouillés qui livrent la majorité du mobilier (fig. 18) et celles issues de diagnostic. Les effectifs disponibles sont ainsi très variables d'un site à l'autre : les sites de Loison-sous-Lens et de Languevoisin-Quiquery, avec plus de 1000 pièces étudiées pour le premier et de presque 2000 pour le second, servent très clairement de référence. À l'inverse, les sites de Blangysur-Bresle, représenté par de seuls ramassages de surface, et celui d'Étouvie dont le mobilier provient essentiellement d'une couche archéologique non homogène, ne seront évoqués que ponctuellement dans la discussion. Cependant, les conditions de découvertes ne sont pas les éléments explicatifs à des effectifs faibles. En effet, à Vermand, la pauvreté du mobilier (107 artefacts pour sept fosses) est la conséquence d'une érosion importante alors qu'à Vitry-en-Artois (131 artefacts pour trois fosses), d'autres arguments, comme la fonction du site, peuvent être évoqués pour interpréter la rareté des artefacts en silex dans les trois fosses intégralement fouillées. Ainsi, la découverte de 163 pièces en silex dans un quart de fosse testée à Sancourt laisse présager la richesse de la structure et permet de considérer la série comme un échantillon utilisable.

\section{Origine, Qualité et ChOIX des matières Premières}

Quel que soit le secteur étudié, le substrat géologique comporte des formations crétacées qui renferment de la matière première siliceuse (fig. 19). Ainsi, les sites disposent d'affleurements potentiellement exploitables dans un rayon de moins de $5 \mathrm{~km}$.

Dans le secteur de Boves/Étouvie, les prospections menées par J. Fabre dans le cadre du PCR " Géoarchéologie du silex » (Fabre 2007) ont permis d'échantillonner différents horizons géologiques comme le Coniacien à Boves même, à proximité immédiate du site sur la même rive gauche de l'Avre, mais aussi du Campanien un peu au sud à Mamont et du Santonien en amont de la vallée de la Noye. La matière première se présente sous la forme de nodules à matrice de couleur grise à noire, hétérogène à faciès peu chargé à chargé d'inclusions grises plus grenues. Le cortex infra à millimétrique est beige, mais de nombreuses diaclases à patine blanche ancienne indiquent un ramassage sans doute en position secondaire. La matière première débitée à Boves ressemble en tout point à celle décrite précédemment.

Dans le secteur de Languevoisin-Quiquery et Sancourt, zone de confluence entre la Somme et l'Ingon, les niveaux de craie à silex du Santonien sont sous-jacents à ceux de la craie Campanienne réputés pauvres en silex. Ils affleurent au nord sur les versants de la vallée de la Somme et recèlent eux des rognons de silex en quantité un peu plus abondante. Ce secteur n'a pas fait l'objet de prospections et aucun référentiel n'est disponible. De couleur noir à gris, le silex taillé sur les deux sites présente de nombreuses inclusions de couleur gris souris à grain un peu plus grenu. Le cortex blanchâtre est souvent épais de quelques millimètres, mais certains petits rognons ont des cortex minces et usés. La qualité de la matière première reste moyenne ainsi qu'en témoignent de nombreux débris dans les assemblages. Par ailleurs, les dimensions relevées sur les blocs en particulier semblent indiquer une matière première aux dimensions plutôt restreintes. Des observations similaires ont été faites pour la série de Vermand, implanté sur les horizons du Coniacien/ Santonien. La matière première est de qualité moyenne mais elle se caractérise surtout par des cortex verdis caractéristiques des horizons du Thanétien, présents à proximité immédiate du site. Cette matière première est identifiée également sur les deux sites précédents, mais en proportion discrète sans doute liée au fait qu'aucun affleurement de ces niveaux n'existe à proximité.

Enfin, dans le Nord de la France, les cartes géologiques (Béthune et Douai) indiquent de vastes affleurements de craie blanche à silex du Sénonien recouverte plus ou moins largement de limons pléistocènes. Dans le secteur de Vitry-en-Artois, le relief très mou n'a pas permis de récolter de matière première en place, par contre, une carrière à Pont-à-Vendin à environ $5 \mathrm{~km}$ au nord-est de Loison-sous-Lens a montré la présence de silex dans ces horizons géologiques (Fabre 2007). Ces silex se présentent sous la forme de nodules à cortex blanc le plus souvent inframillimétrique mais pouvant être supramillimétrique, à 


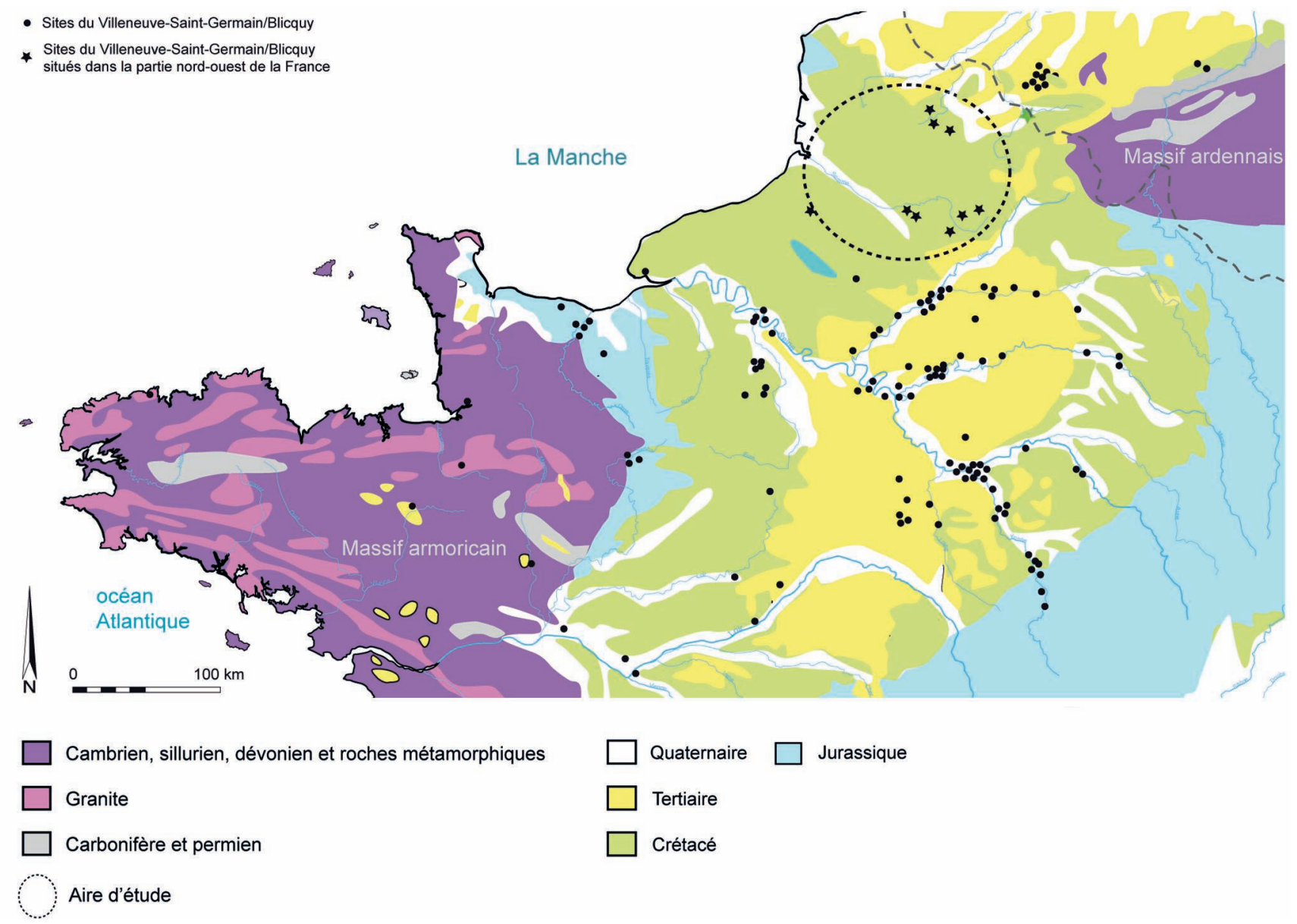

Fig. 19 - Carte géologique simplifiée de la moitié nord de la France et localisation de l'aire d'étude (Carte géologique de la France. Classe de 1e D.T., t. II : Géologie, Paris, Bordas, 1972 ; I. Praud, Inrap). Geological map of the northern half of France (after Bordas 1972) and location of the studied area (I. Praud, Inrap).

matrice hétérogène à macrofaciès peu chargé. Ici aussi, la présence de cortex verdi sur certains artefacts confirme un ramassage d'une partie des rognons dans ces horizons.

Ainsi, sur l'ensemble des sites, la préférence a été donnée aux matériaux d'origine locale disponibles le plus souvent à proximité immédiate de l'habitat. La présence d'éclats totalement corticaux indique que les rognons ont été rapportés sous forme brute.

Cependant, si les silex locaux sont clairement dominants (fig. 20), on note l'apport systématique de silex considérés comme non locaux, même si certaines origines restent indéterminées à ce jour. Parmi ces matériaux non locaux, on soulignera la très nette suprématie du silex tertiaire Bartonien du Bassin parisien (fig. 21) identifiable par sa couleur brune parfois zonée et comportant des fossiles caractéristiques (oogones de characées). Il représente $83 \%$ des artefacts importés et est présent sur cinq des sept sites étudiés, également sur le site d'Étouvie (Prodéo et al. 1997). Si son absence à Sancourt est sans doute liée au caractère très limité des tests dans les structures néolithiques, celle de Vitry-en-Artois revêt une signification autre sur laquelle nous reviendrons ensuite. En tout état de cause, la place prépondérante du silex Bartonien au sein des réseaux de diffusion du BVSG (Bostyn 1994 et 1997,
Allard et Bostyn 2006, Bostyn et Denis 2016) est une nouvelle fois observée, renforcée par sa présence au sein de séries numériquement faibles et sur des sites localisés en périphérie des zones de forte densité d'occupation.

Parmi les autres matériaux, on évoquera la diversité des origines qui ne sont pas les mêmes en fonction des sites (fig. 22). Sur le site de Vitry-en-Artois, on note la présence de silex turonien de l'Escaut qui suppose un déplacement d'environ $30 \mathrm{~km}$ vers le sud-est. À Vermand, on a identifié du silex de Ghlin venant du bassin de Mons (Belgique) localisé à environ $85 \mathrm{~km}$ au nord alors que sur le site de LanguevoisinQuiquery deux artefacts en silex blond translucide viennent probablement de la région champenoise. La faible représentation du silex de Ghlin dans les séries étudiées doit être soulignée dans la mesure où ce silex a été identifié dans la vallée de l'Oise (Bostyn 1994, Bostyn et al. 2012) et dans la vallée de l'Aisne (Allard 1999, Denis 2014a), nettement plus éloignées des sources. La forte implication de cette matière première dans l'économie lithique de Hesbaye (Denis 2014b) est probablement un élément explicatif de cette relativement faible diffusion vers l'ouest et le sud. Ainsi, l'identification de l'origine des matériaux non locaux montre une structuration des réseaux de 


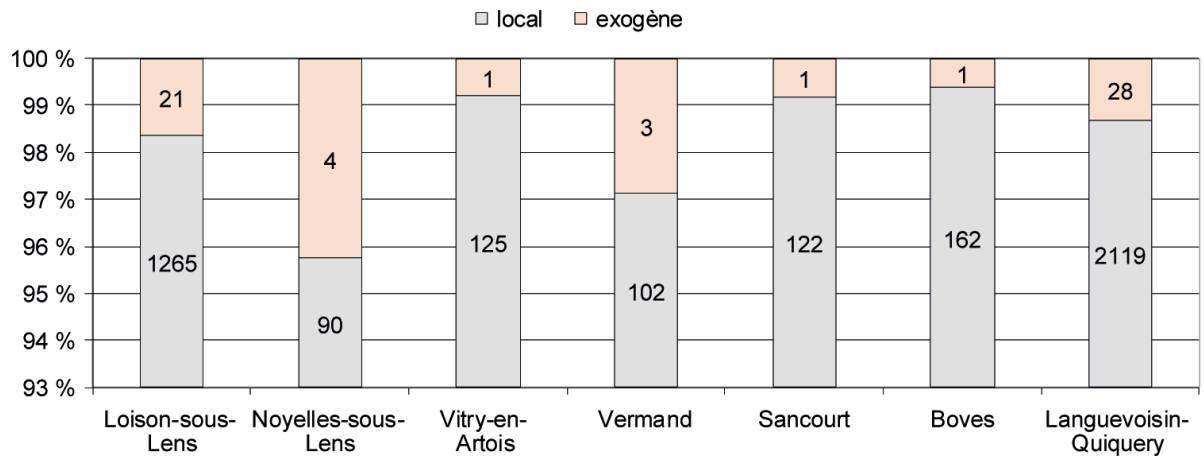

Fig. 20 - Part des matériaux locaux et exogènes ( $F$. Bostyn, Inrap).

Distribution of local and exogeneous raw materials (F. Bostyn, Inrap).

Fig. 21 - Différentes origines des matériaux non locaux (F. Bostyn, Inrap). TB : tertiaire Bartonien ; Gh : Ghlin ; Tu Es : turonien de l'Escaut ; BI : silex blond; Ind : indéterminé.

Different origins of the non-local raw materials (F. Bostyn, Inrap).

TB: Tertiary Bartonian; Gh: Ghlin; Tu Es: Turonian from the Scheldt valley; Bl: blond flint; Ind: non-determined.
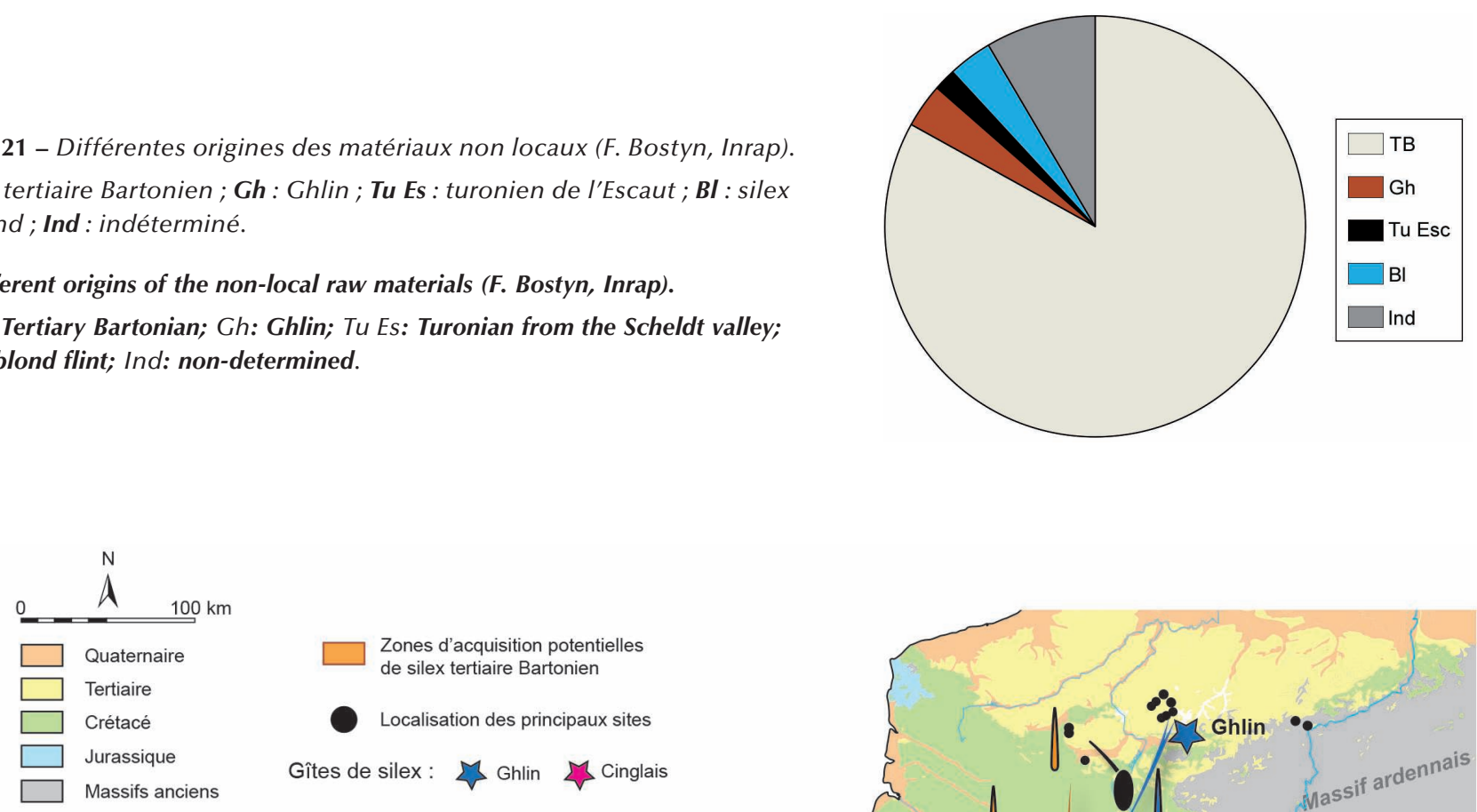

Gîtes de silex : $M$ Ghlin $M$ Cinglais
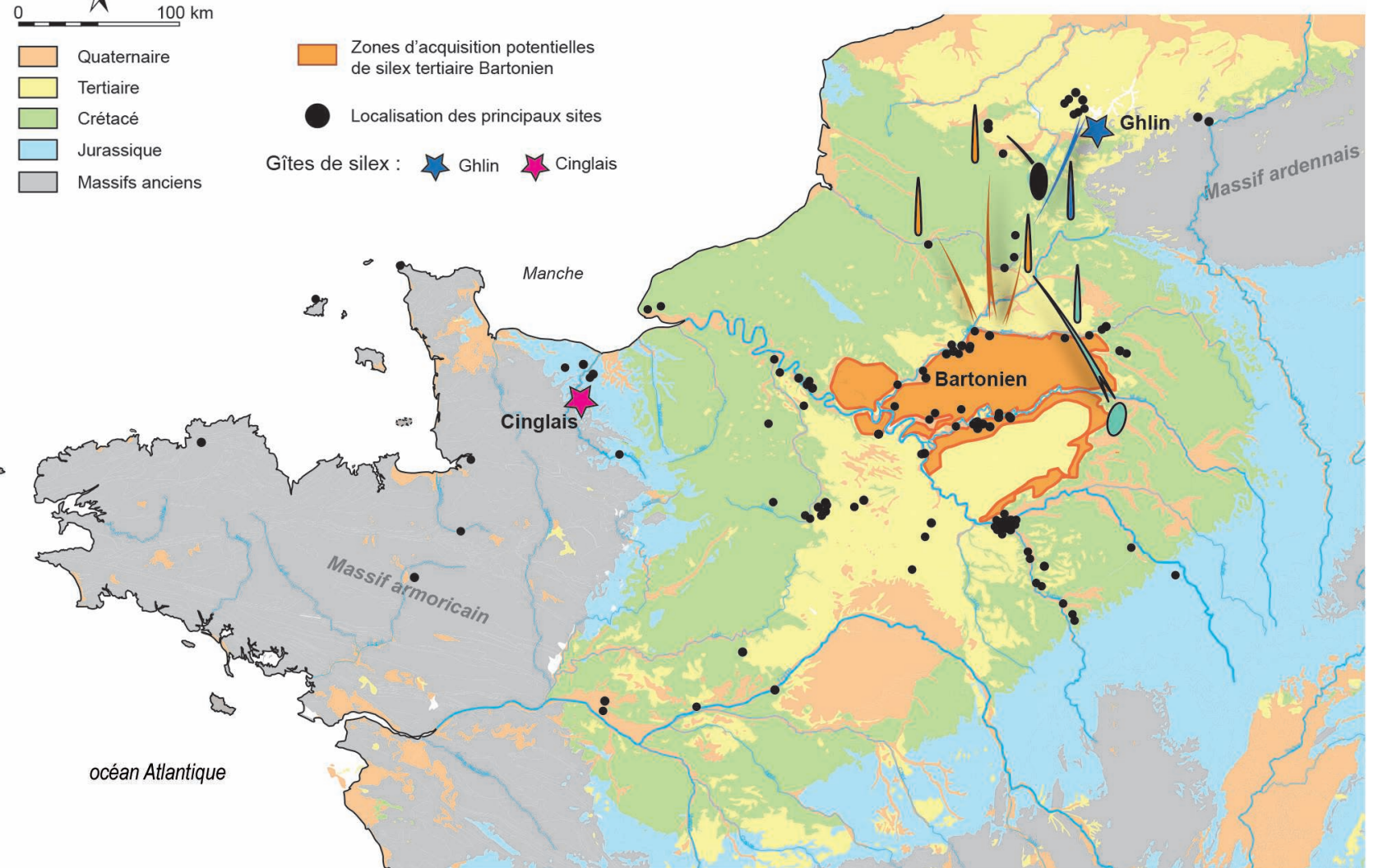

Fig. 22 - Cartographie des régions d'origine des matériaux siliceux non locaux identifiés (fond de carte N. Fromont, Inrap ; DAO F. Bostyn, Inrap).

Mapping of the sources of the non-Iocal siliceous materials (base map N. Fromont, Inrap ; CAD F. Bostyn, Inrap). 
circulation à différentes échelles, avec une superposition des différents réseaux de diffusion des matériaux certains ayant un rayonnement régional (entre 50 et $80 \mathrm{~km}$ ) alors que d'autres (Bartonien) ont un impact sur l'ensemble du territoire occupé par la culture BVSG avec une diffusion au-delà de $100 \mathrm{~km}$.

\section{Productions domestiQues, Productions importées : DES RÉPONSES AUX BESOINS DIVERSIFIÉES}

Sur l'ensemble des sites, une production d'éclats a été réalisée sur place sur les matériaux locaux. Les analyses de ces productions basées sur la confrontation entre les déchets de taille (éclats et nucléus) et les outils sur éclat montrent des productions à faible investissement technique (percuteur dur en percussion directe) et réalisées selon des schémas opératoires peu élaborés. Le débitage est unipolaire lorsque le rognon a fait l'objet d'une faible exploitation et multidirectionnel lorsque le volume initial a permis plusieurs phases de débitage. L'examen de ces nucléus atteste de séries d'enlèvements unipolaires successifs permettant une utilisation maximale des volumes (fig. 23). La comparaison entre les supports bruts et retouchés entre les deux sites ayant fourni des assemblages suffisants (fig. 24), montre que les outils ont été fabriqués aux dépens des supports les plus longs et plutôt allongés (la longueur étant entre une et deux fois la largeur). Cependant, et c'est observable dans les deux cas, certains artefacts sont nettement plus larges que longs (longueur inférieure à la largeur), mais en l'état actuel des recherches, aucune véritable chaîne opératoire spécifique de production de ces supports n'a pu être identifiée. On perçoit également dans ces graphiques, l'impact de la faible dimension des rognons débités à Languevoisin-Quiquery concentrant la très grande majorité des artefacts en dessous de $6 \mathrm{~cm}$ de longueur. La présence de ce type de support est notée à Boves, mais pas à Sancourt, pas plus qu'à Vitry-en-Artois où aucun des éclats retrouvés ne correspond à une telle production.
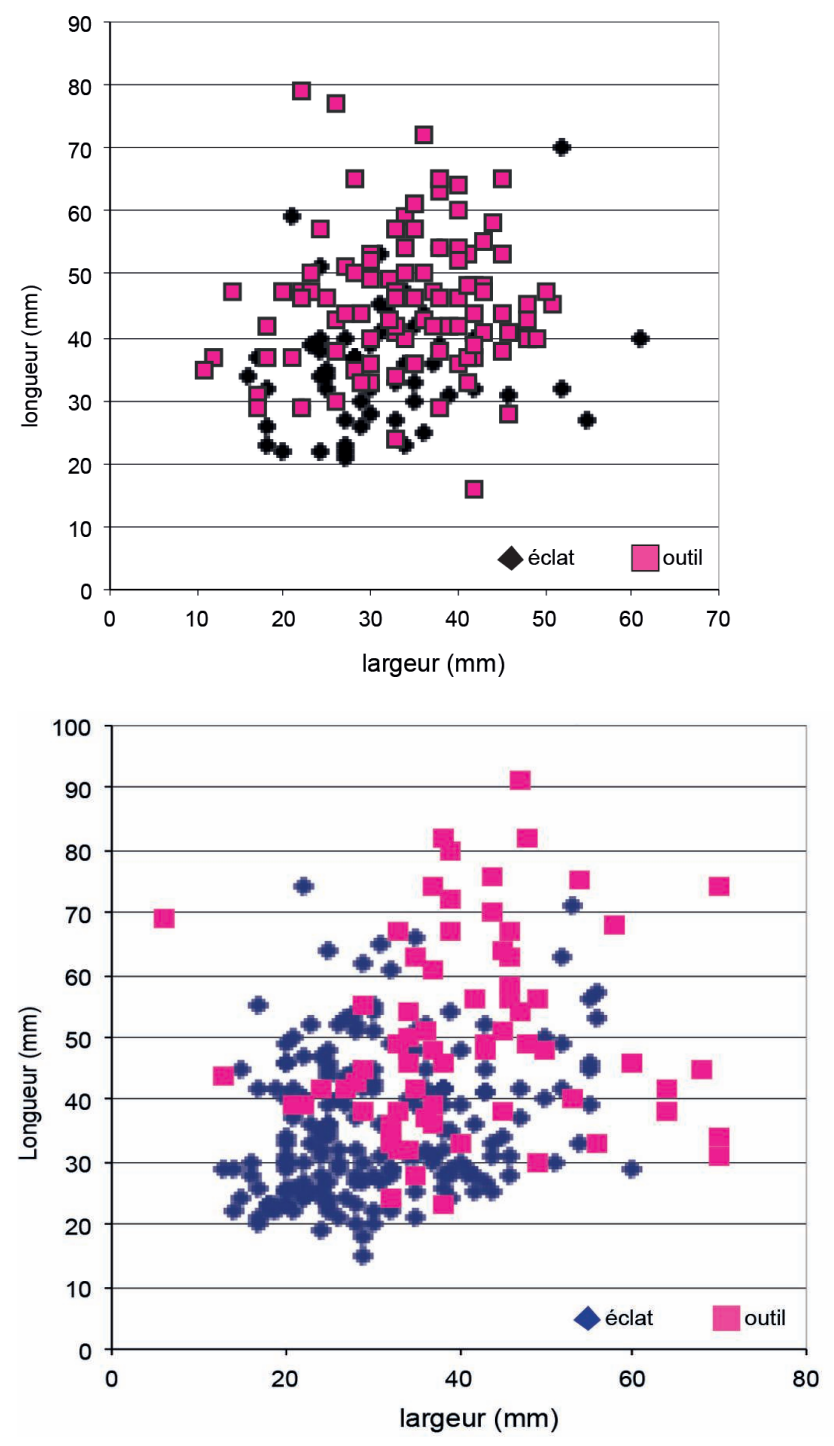

Fig. 24 - Comparaison des dimensions des éclats et outils sur éclats entre les sites de Languevoisin (1) et de Loison-sous-Lens ( 2 ; F. Bostyn, Inrap).

Comparison of flake and tool dimensions from the sites of Languevoisin (1) and Loison-sous-Lens (2; F. Bostyn, Inrap).

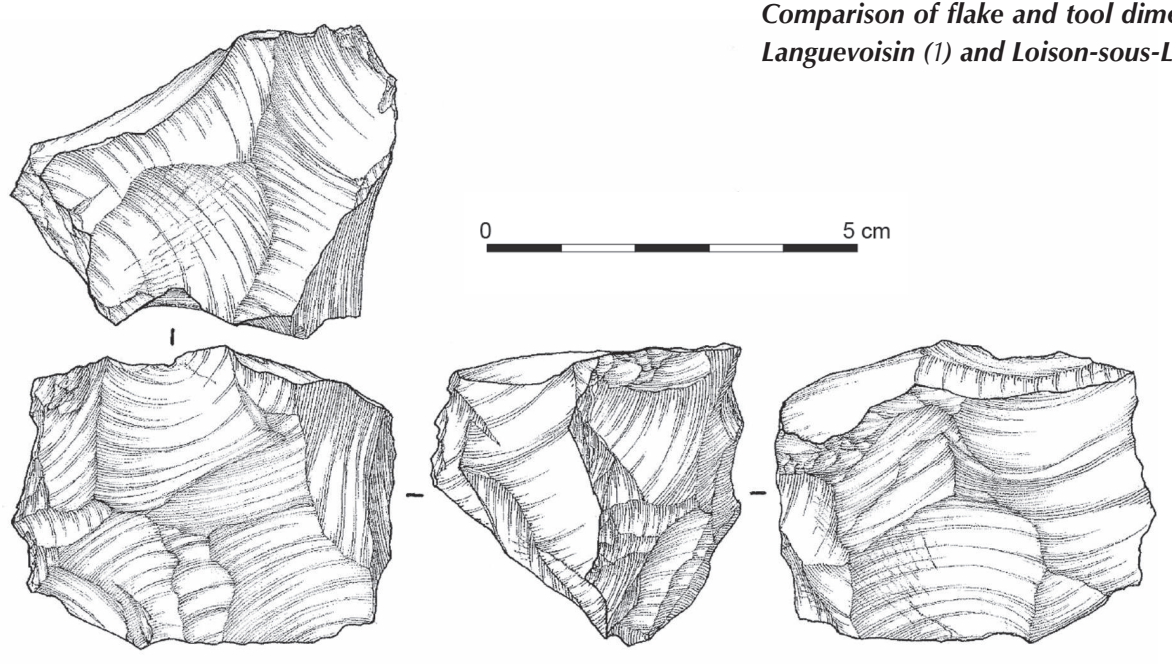

Fig. 23 - Nucléus à débitage multidirectionnel de Languevoisin-Quiquery (È. Boitard, Inrap). Core with multidirectional knapping from Languevoisin-Quiquery (̇̀. Boitard, Inrap). 
L'autre composante des séries lithiques est la production laminaire, mais ici, d'importantes différences sont visibles entre les assemblages, à la fois parce que plusieurs matériaux, pas forcément locaux, entrent en jeu dans cette production et que, de surcroît, elle n'est pas forcément réalisée au sein des habitats. Le site de Loison-sous-Lens constitue la meilleure série pour aborder les schémas opératoires de production. Elle a été réalisée, en effet, pour les matériaux locaux, le silex crétacé, dans son intégralité sur place. La mise en forme des rognons comprend la mise en place d'une crête antérieure servant de guide à la première lame. Le débitage est unipolaire ainsi qu'en témoignent les faces supérieures des lames. La création du plan de frappe s'est faite par l'enlèvement d'un gros éclat perpendiculairement à l'axe de débitage envisagé, mais le plan de frappe a été préparé pour chaque extraction de lame par des petits enlèvements centripètes légèrement concaves. Le réfléchissement régulier de ces enlèvements a entraîné la suppression de tablettes complètes pour recréer des angles de débitage favorables. Quelques déchets caractéristiques montrent que l'entretien des surfaces laminaires a été réalisé par le biais d'enlèvements à partir de la surface laminaire et perpendiculairement à l'axe de débitage. La présence de lames à un pan dont l'un des flancs est totalement cortical témoigne d'une ouverture de la surface de débitage sur des bords restés bruts. Le seul nucléus conservé a été malheureusement fortement redébité et réutilisé en percuteur, et ne fournit que peu d'indications sur les chaînes opératoires. L'extraction des lames s'est faite par percussion indirecte. Les produits obtenus à Loison-sous-Lens sont des lames d'environ $8 \mathrm{~cm}$ de long pour une largeur moyenne de $1,8 \mathrm{~cm}$ (fig. 25, no 9 et 11). Cette chaîne opératoire est assez commune et a été décrite à de nombreuses reprises pour le BVSG du Bassin parisien (Bostyn 1994 et 2009, Bostyn et al. 2003, Allard 2005, Allard et Bostyn 2006, Denis 2014a). Sur le site de Languevoisin-Quiquery, malgré une série assez abondante, on n'a pu identifier que très peu de déchets caractéristiques de cette production en dehors des lames et outils sur lames (fig. 25, no 3 et 14). On se propose d'interpréter cette carence comme le témoin d'une introduction sur le site de nucléus à lame déjà préformés, voire déjà partiellement exploités. Sur les sites de Noyelles-sous-Lens, Vitry-enArtois, Boves et Vermand, la présence de différents artefacts se rapportant à la chaîne opératoire laminaire laisse envisager

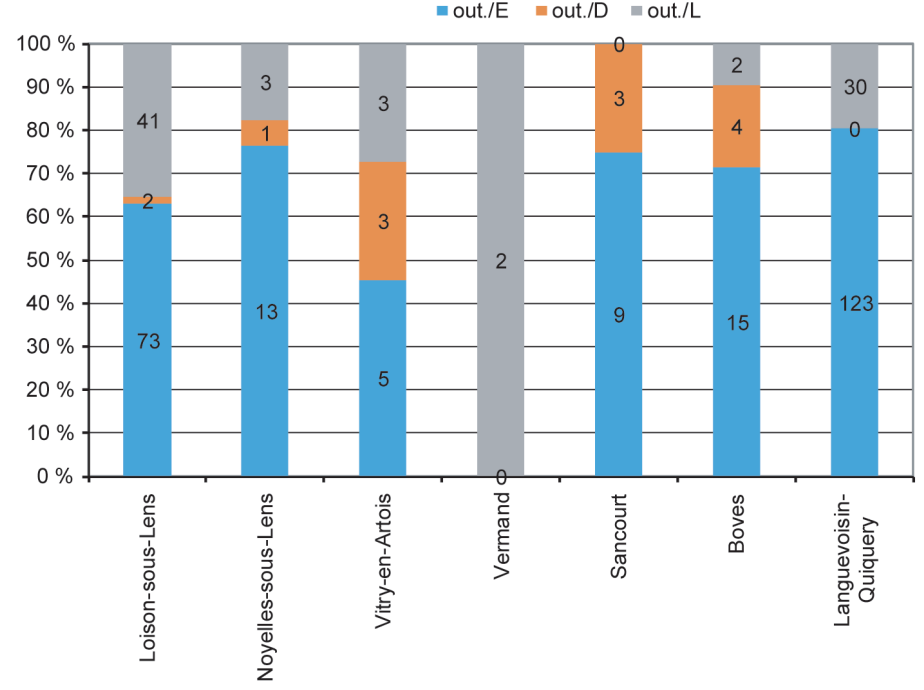

Fig. 26 - Part des différents types de supports dans l'outillage par site (F. Bostyn, Inrap).

Proportions of the different types of tool blanks at the different sites (F. Bostyn, Inrap).

la réalisation de cette production sur place, au moins partiellement, mais les faibles effectifs globaux incitent à la prudence. Signalons enfin que seul le site de Loison-sous-Lens a fourni quelques témoins de l'utilisation de la technique du microburin pour la fracturation des lames.

La production laminaire, en dehors du site de Loison-sousLens, n'est donc pas au centre des productions domestiques sur les matériaux locaux. Cette carence pourrait s'expliquer par un manque de savoir-faire sur les différents sites, mais aussi par des natures ou des fonctions différentes des sites, hypothèses que nous discuterons par la suite. Néanmoins, le recours aux supports laminaires est systématique dans l'outillage, où ils représentent entre 10 et $40 \%$ des supports sélectionnés (fig. 26), et, à l'exception des sites de Vitryen-Artois et de Sancourt, l'apport de produits laminaires en matériaux non locaux est une pratique commune (fig. 27). Dans la quasi-totalité des cas d'ailleurs, les matériaux non locaux n'ont été introduits que sous la forme de produits

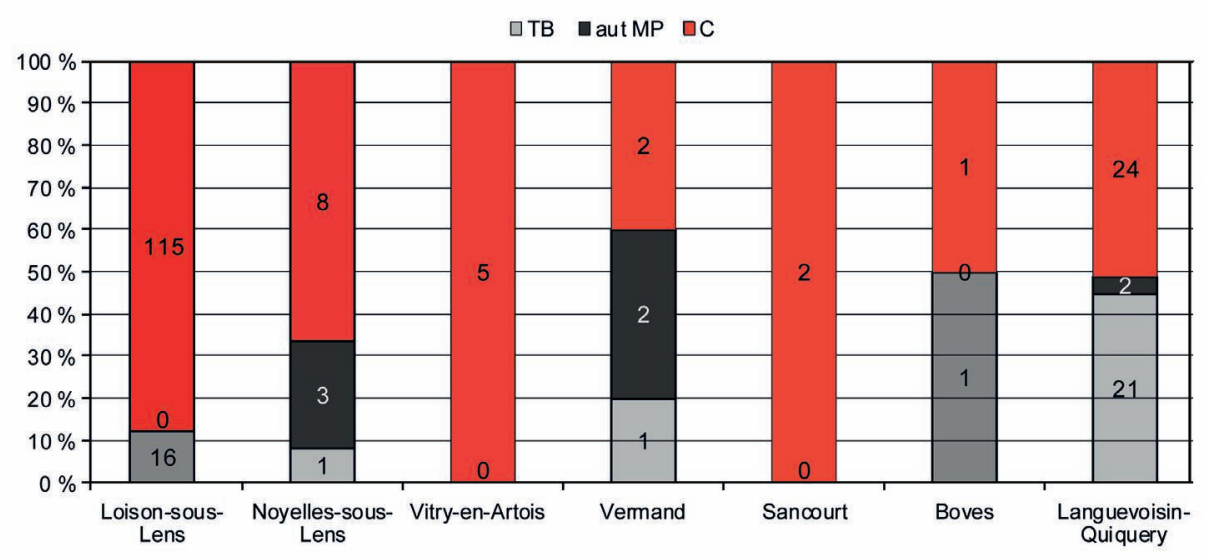

Fig. 27 - Origine des matières premières représentées dans les produits laminaires, outillage et produits bruts confondus (F. Bostyn, Inrap).

C: crétacé, aut. MP: autres matières premières, $\boldsymbol{T B}$ : tertiaire bartonien.

Sources of the raw materials represented in the blade tool, tool and unretouched blank categories (F. Bostyn, Inrap).

$C$ : Cretaceous flint; aut MP: other raw materials; TB: Tertiary Bartonian. 

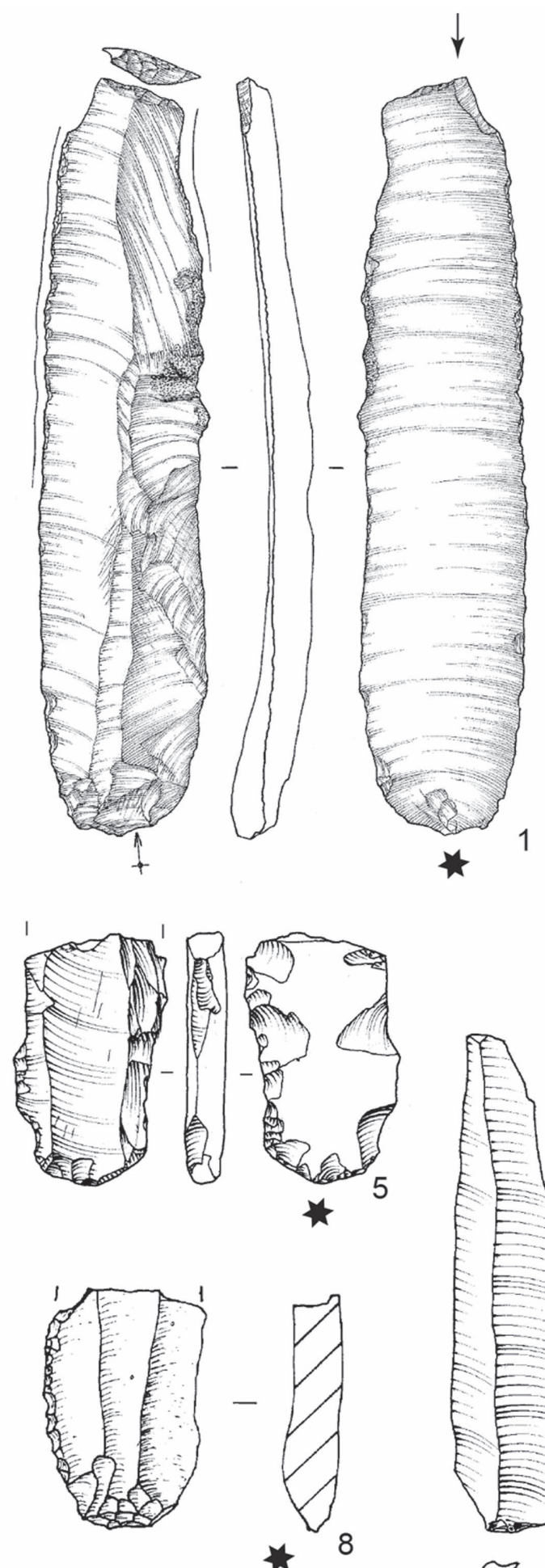

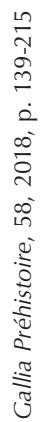

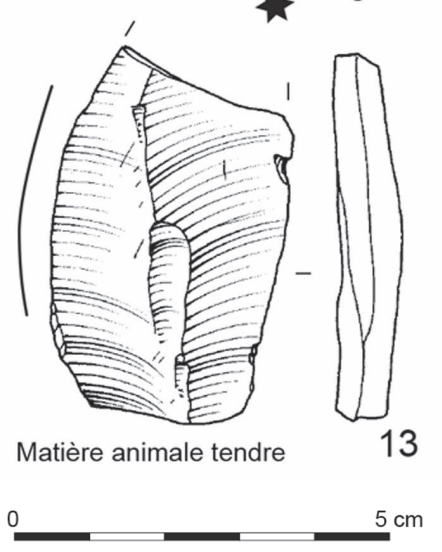

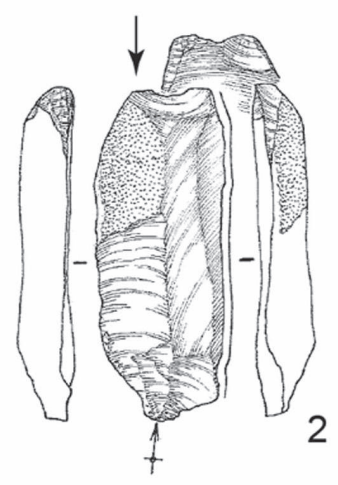
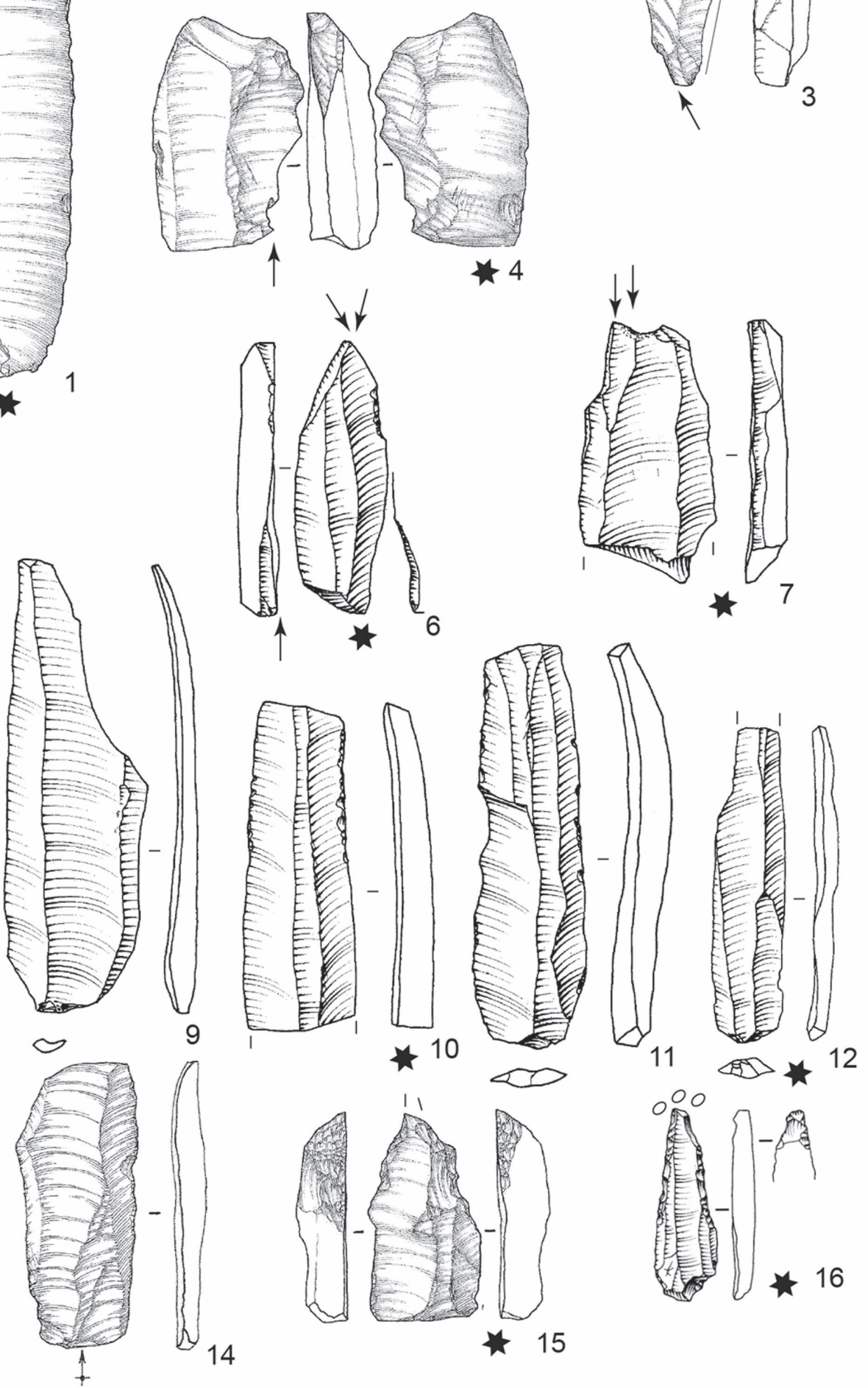

Silex bartonien 
4 Fig. 25 - 1-4 et 6-7. burins sur lames; 5 et $\mathbf{8}$. lames retouchées; 9 et 12. lames brutes; 10-11 et 13-14. lames utilisées; 15-16. perçoirs.

1-4. Languevoisin (È. Boitard, Inrap) ; 5. Loison-sous-Lens (S. Lancelot, Inrap) ; 8. Vermand (F. Bostyn, Inrap) ; 9-13 et 16. Loison-sous-Lens (S. Lancelot, Inrap) ; 14-15. Languevoisin (È. Boitard, Inrap).

1-4 and 6-7. burins on blades; 5. retouched blades; 8. retouched blades; 9 and 12. non-retouched blades; 10-11 and 13-14. used blades; 15-16. perforators.

1-4. Languevoisin (ל̀. Boitard, Inrap); 5-7. Loison-sous-Lens (S. Lancelot, Inrap); 8. Vermand (F. Bostyn, Inrap); 9-13 and 16. Loison-sous-Lens (S. Lancelot, Inrap); 14-15. Languevoisin (ì. Boitard, Inrap).

laminaires. Seul le silex turonien de l'Escaut a été introduit à Vitry-en-Artois sous la forme d'un éclat ou d'un outil. Très logiquement (fig. 21), le silex bartonien est le plus représenté dans les matériaux importés et il représente $12,2 \%$ des produits laminaires à Loison-sous-Lens. Il apparaît même en quantités équivalentes au silex local à LanguevoisinQuiquery. Parmi les sept autres produits laminaires importés, quatre sont dans des matières premières non identifiées, les deux de Vermand sont originaires du bassin de Mons et celle de Languevoisin-Quiquery proviendrait de Champagne, ce qui montre des réseaux d'approvisionnements différenciés, reliant le site de Vermand aux sites du Nord et du bassin de Mons, alors que celui de Languevoisin-Quiquery serait en relation plutôt avec les sphères plus "méridionales " du BVSG. Ainsi, si le silex bartonien apparaît comme une matière première d'unité de la culture BVSG, les autres matériaux ont des résonances plus régionales.

L'une des interrogations soulevées par la présence de ces produits importés sur les sites, concerne les raisons de cet apport. Des éléments de réponse peuvent être apportés par l'analyse qualitative des artefacts. Si l'on prend en considération l'ensemble des objets importés, on observe que les lames sont des produits réguliers, à bords et nervures parallèles relevant de productions soignées (fig. $25, \mathrm{n}^{\circ} 1,7$ et 10). Dans la grande majorité des cas, il s'agit de lames de plein débitage, nous n'avons identifié que quatre lames sous crête (fig. 33, $\mathrm{n}^{\circ} 2$ ), et essentiellement non corticales, seules quatre présentent des résidus corticaux marginaux. Deux lames présentent néanmoins un bord cortical continu, témoin d'un procédé d'ouverture latérale de la surface laminaire (fig. 33, $\mathrm{n}^{\circ} 1$ ). Quant aux dimensions, il est plus difficile de se prononcer, puisque rares sont les lames entières. Pour le silex bartonien, deux fragments provenant du site de LanguevoisinQuiquery nous indiquent une longueur avoisinant sans doute les $15 \mathrm{~cm}$ de long, puisqu'ils mesurent 12/13 cm fragmentés. Les largeurs moyennes sont de $20,1 \mathrm{~mm}$ pour les produits en bartonien contre $17,5 \mathrm{~mm}$ pour le crétacé et les épaisseurs respectivement de $5,6 \mathrm{~mm}$ pour $4,6 \mathrm{~mm}$. Les lames en Bartonien sont donc aussi plus larges et plus massives. Les dimensions des autres lames sont, elles, conformes à celles des lames en silex crétacé, ce qui confirme que l'apport de lames en silex bartonien répond bien à des besoins particuliers auxquels les matériaux locaux ne peuvent subvenir.

\section{L'OUTILLAGE}

Les sept sites étudiés (tabl. 5) ont livré une série de 252 outils sur éclats et débris (soit $75,7 \%$ ) et 81 outils sur lames (soit 24,3\%) et sans grande surprise ce sont les sites de Loison-sous-Lens et de Languevoisin-Quiquery qui rassemblent le plus d'outils. Néanmoins, les vingt-deux outils du site de Boves et les quatorze outils de Noyelles constituent des échantillons non négligeables au vu du nombre de structures fouillées. Pour le site de Vitry-en-Artois par contre, le fait de disposer de seulement onze outils pour trois structures intégralement fouillées est significatif du caractère particulier de l'occupation. Pour les sites de Sancourt et Vermand, ne disposant soit d'aucun outil sur lame, soit d'aucun outil sur éclat, les séries ne sont guère représentatives mais néanmoins intéressantes dans leur composition. Très logiquement aussi, ce sont les matières premières locales (Crétacé et Thanétien) qui sont sélectionnées comme support d'outils.

Parmi les outils sur éclat, les éclats retouchés et les denticulés sont les plus représentés, atteignant 28,2 \% chacun, et ceci sur tous les sites. Les denticulés sont réalisés sur des éclats de dimensions très variables qui figurent cependant parmi les plus épais (fig. 29). Les éclats d'entame sont ainsi souvent recherchés. Les retouches sont constituées de coches successives, larges et abruptes soit directes (fig. 28, $\mathrm{n}^{\circ} 1$ et 5) soit bifaciales (fig. $28, n^{\circ} 3-4$ ). Les coches sont soit de larges coches clactoniennes, soit des encoches plus étroites à retouche abrupte plutôt marginale souvent accompagnées de retouches fines du bord.

Les grattoirs avec 9,5\% constituent la troisième catégorie d'outils sur éclat. Les études des supports semblent indiquer la recherche d'éclats de forme plutôt allongée - dont les épaisseurs semblent moindres que celles des denticulés - concentrée entre 6 et $15 \mathrm{~mm}$. Les fronts sont la plupart du temps situés en partie distale du support et ont été confectionnés par retouche directe abrupte (fig. 30, $\mathrm{n}^{\circ} 1$ ). Dans quelques cas, une retouche sur l'un des bords accompagne la retouche frontale. L'état de la face supérieure ne semble pas constituer un critère déterminant puisque l'on trouve tous les cas de figure depuis les entames (fig. 30, no 3 ) jusqu'aux éclats non corticaux. Dans trois cas à Languevoisin-Quiquery, on a noté la présence de retouches inverses sur le front, permettant de classer ces outils dans la catégorie des grattoirs-herminettes (Caspar et Burnez-Lanotte 1996).

Deux catégories d'outils arrivent ensuite, les burins $(6,7 \%)$ et les tranchets $(6,3 \%)$. Les burins, au nombre de 17 au total, sont de types très variés - sur cassure d'angle, sur troncature distale, sur le talon, dièdre - et de morphologies diversifiées ne permettant pas de faire un résumé, chaque outil étant unique. Dans deux cas, des lustrés brillants sont observés sur les pans des burins, dans deux autres cas, le bord opposé au pan présente une fine retouche marginale d'utilisation. Aucun critère ne semble avoir prévalu dans la sélection des supports. Les tranchets au nombre de 16 proviennent exclusivement des sites de Loison-sous-Lens, de Vitry-en-Artois et de Boves. Les tranchets ont été réalisés sur deux types de supports : des éclats allongés et des éclats larges (fig. 31). Dans le cas des éclats 
Tabl. 5 - Décompte de l'outillage par site et par matières premières (F. Bostyn, Inrap).

$\boldsymbol{A}$ : sur éclat / débris ; $\boldsymbol{B}$ : sur lame. $\boldsymbol{T B}$ : tertiaire bartonien ; $\boldsymbol{C}$ : crétacé ; Ind : indéterminé ; Sbl : secondaire blond ; Gh : ghlin ; CN : crétacé noir. Toolkit counts by site and raw material (F. Bostyn, Inrap).

A: on flakes/debris; B: on blades. TB: Tertiary Bartonian; C: Cretaceous flint; Ind: non-determined; Sbl: secondary blond; Gh: Ghlin; CN: black Cretaceous flint.

\begin{tabular}{|c|c|c|c|c|c|c|c|c|c|c|c|c|c|c|c|}
\hline \multirow{2}{*}{$\begin{array}{c}\text { Site } \\
\text { Type MP }\end{array}$} & \multirow{2}{*}{$\frac{\text { Loison }}{\text { C }}$} & \multirow{2}{*}{$\begin{array}{c}\text { Noyelles } \\
\text { C }\end{array}$} & \multicolumn{3}{|c|}{ Vitry } & \multicolumn{2}{|c|}{ Sancourt } & \multirow{2}{*}{$\begin{array}{c}\text { Boves } \\
\text { C }\end{array}$} & \multicolumn{2}{|c|}{ Languevoisin } & \multicolumn{3}{|c|}{ Total / MP } & \multirow{2}{*}{ Tot } & \multirow{2}{*}{$\%$} \\
\hline & & & Es & Th & C & Th & C & & Th & C & Es & Th & C & & \\
\hline grattoir & 12 & & & & & 1 & 2 & 1 & 2 & 6 & 0 & 3 & 21 & 24 & 9,5 \\
\hline denticulé & 15 & 2 & & 1 & 3 & 1 & 4 & 4 & 5 & 36 & 0 & 7 & 64 & 71 & 28,2 \\
\hline éclat retouché & 24 & 4 & & & 2 & & 2 & 5 & & 35 & 0 & 0 & 72 & 72 & 28,6 \\
\hline éclat utilisé & & & & & & & & 3 & & 11 & 0 & 0 & 14 & 14 & 5,6 \\
\hline burin & 5 & 2 & & & & & & 2 & & 8 & 0 & 0 & 17 & 17 & 6,7 \\
\hline tranchet & 10 & & 1 & & & & & 5 & & & 1 & 0 & 15 & 16 & 6,3 \\
\hline perçoir & & & & & & & & & & 3 & 0 & 0 & 3 & 3 & 1,2 \\
\hline grat-herminette & & & & & & & & & & 3 & 0 & 0 & 3 & 3 & 1,2 \\
\hline éclat émoussé & 1 & 1 & & & & & & & & & 0 & 0 & 2 & 2 & 0,8 \\
\hline racloir / denticulé & 1 & & & & & & & & & & 0 & 0 & 1 & 1 & 0,4 \\
\hline débris retouché & 1 & 1 & & & & & & & 1 & 2 & 0 & 1 & 4 & 5 & 2,0 \\
\hline pièce esquillée & 2 & & & & & & & & & 2 & 0 & 0 & 4 & 4 & 1,6 \\
\hline coche & & & & & & & & & 1 & & 0 & 1 & 0 & 1 & 0,4 \\
\hline éclat percuté & & & & & & & & & & 1 & 0 & 0 & 1 & 1 & 0,4 \\
\hline faucille & & 1 & & & & & & & & 1 & 0 & 0 & 2 & 2 & 0,8 \\
\hline troncature & & & & & 1 & & & & & & 0 & 0 & 1 & 1 & 0,4 \\
\hline ébauche bifaciale & & & & & & & 1 & & & & 0 & 0 & 1 & 1 & 0,4 \\
\hline fragment & 4 & 3 & & & & & 1 & & 1 & 5 & 0 & 1 & 13 & 14 & 5,6 \\
\hline total/MP & 75 & 14 & 1 & 1 & 6 & 2 & 10 & 20 & 10 & 113 & 1 & 13 & 238 & 252 & 100 \\
\hline total & 75 & 14 & \multicolumn{3}{|c|}{8} & \multicolumn{2}{|c|}{12} & 20 & \multicolumn{2}{|c|}{123} & \multicolumn{3}{|c|}{252} & & \\
\hline
\end{tabular}

\begin{tabular}{|c|c|c|c|c|c|c|c|c|c|c|c|c|c|c|c|c|c|c|c|c|c|}
\hline \multirow{2}{*}{$\begin{array}{c}\text { Site } \\
\text { Type MP }\end{array}$} & \multicolumn{2}{|c|}{ Loison } & \multicolumn{2}{|c|}{ Noyelles } & \multirow{2}{*}{$\begin{array}{c}\text { Vitry } \\
\text { C }\end{array}$} & \multicolumn{2}{|c|}{ Vermand } & \multicolumn{2}{|c|}{ Boves } & \multicolumn{4}{|c|}{ Languevoisin } & \multicolumn{6}{|c|}{ Total / MP } & \multirow{2}{*}{ Tot } & \multirow{2}{*}{$\%$} \\
\hline & TB & C & TB & Ind & & Gh & TB & TB & C & SBI & $\mathrm{CN}$ & TB & C & Ind & Gh & SBI & $\mathrm{CN}$ & TB & C & & \\
\hline lame retouchée & & 7 & & & & & 1 & & & & & 1 & 3 & & & & & 2 & 10 & 12 & 14,8 \\
\hline burin & 5 & 8 & 1 & 1 & & & & & & 1 & & 8 & 3 & 1 & & 1 & & 14 & 11 & 27 & 33,3 \\
\hline armature & 1 & 6 & & & & 1 & & & & & & & & & 1 & & & 1 & 6 & 8 & 9,9 \\
\hline perçoir & 1 & & & & & & & & & & & 1 & & & & & & 2 & & 2 & 2,5 \\
\hline troncature & 1 & 1 & & & 1 & & & 1 & 1 & & & & & & & & & 2 & 3 & 5 & 6,2 \\
\hline fragment & & 2 & & & & & & & & & & & 1 & & & & & & 3 & 3 & 3,7 \\
\hline total/MP & 9 & 32 & 1 & 2 & 3 & 1 & 1 & 1 & 1 & 1 & 1 & 14 & 14 & 2 & 1 & 1 & 1 & 26 & 50 & 81 & 100 \\
\hline
\end{tabular}



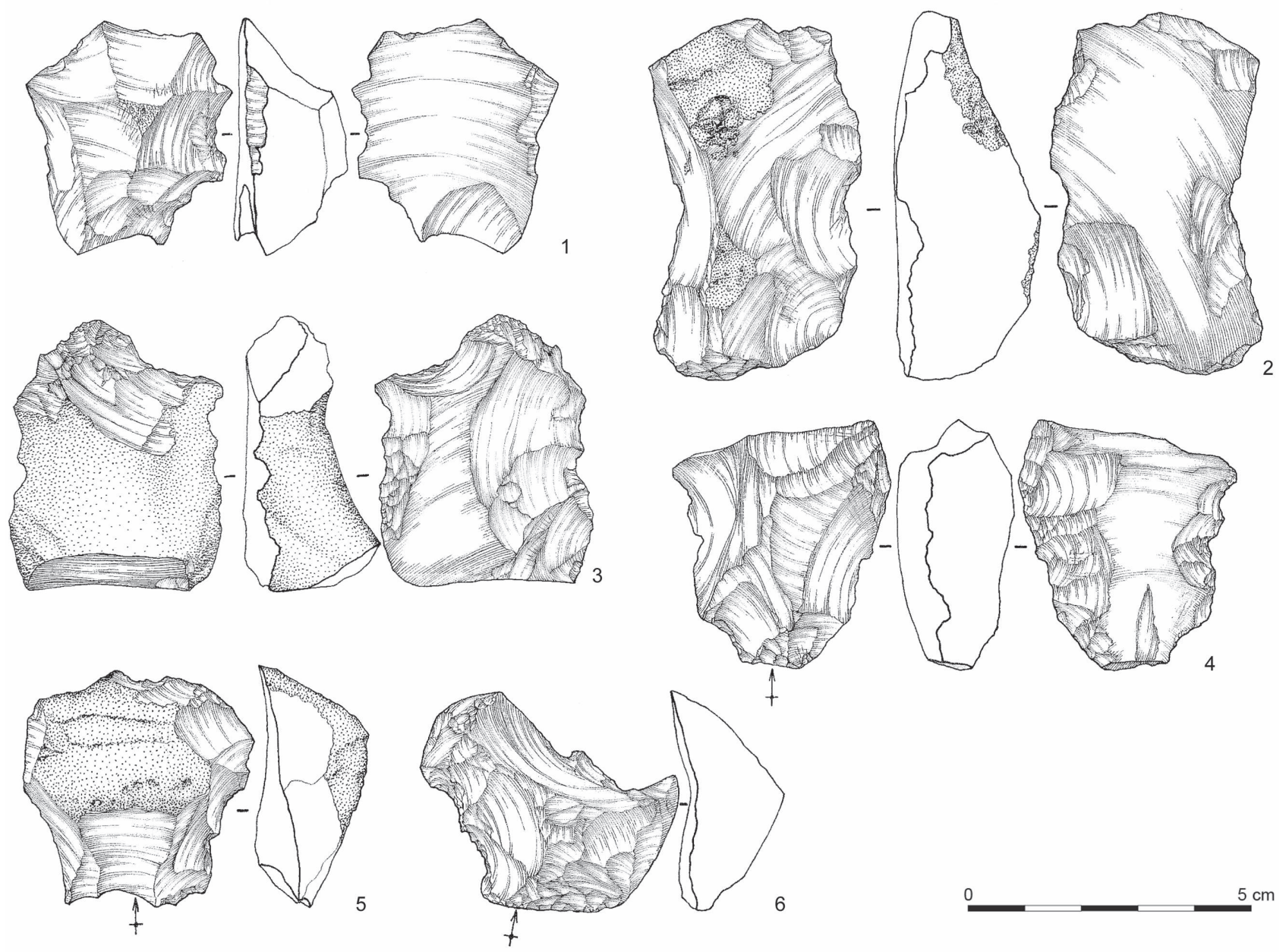

Fig. 28 - Denticulés du site de Languevoisin-Quiquery (È. Boitard, Inrap).

Denticulates from Languevoisin-Quiquery (̇̀. Boitard, Inrap).

Fig. 29 - Comparaison de l'épaisseur des supports sélectionnés pour la fabrication des grattoirs $(\boldsymbol{G})$, des denticulés $(\boldsymbol{D})$ et des tranchets (Tr) du site de Loison-sous-Lens (F. Bostyn, Inrap).

Comparison of the thickness of blanks selected for making end scrapers $(G)$, denticulates $(D)$ and tranchets (Tr) from the Loisons-sous-Lens site (F. Bostyn, Inrap).

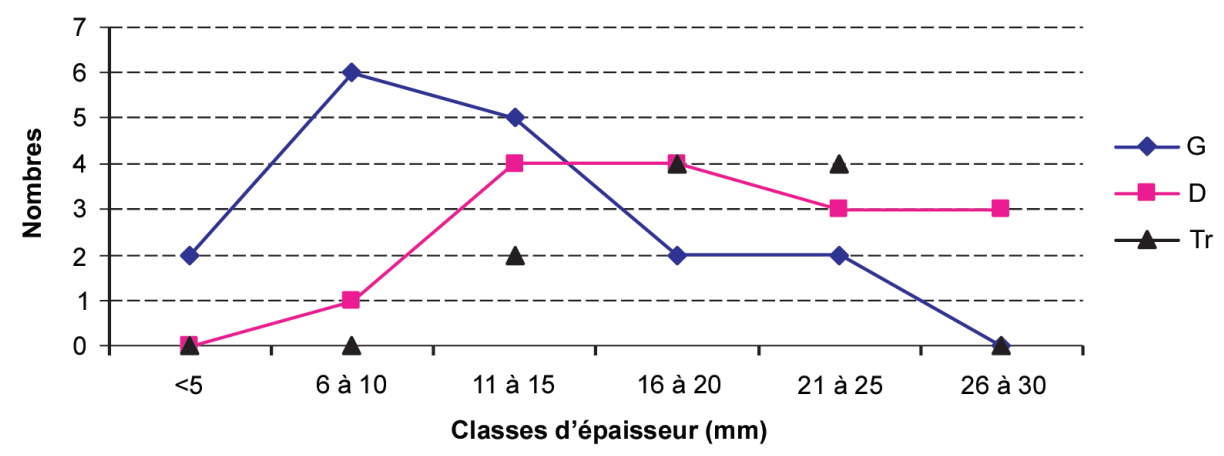

allongés, la partie active se trouve en distal de l'éclat (fig. 32, $\mathrm{n}^{\circ} 1$ et 3-4), la retouche concernant alors les deux bords. La retouche est abrupte, directe ou inverse. La sélection des éclats larges amène le tailleur à utiliser l'un des petits bords comme partie active (fig. $32, \mathrm{n}^{\circ} 2$ et 5). Les extrémités proximales et distales du support sont alors retouchées par retouche abrupte directe ou inverse. Dans ces cas, le bulbe de percussion a été aminci par retouches inverses plates. La partie active présente souvent un esquillement et un lustré très net dans quatre cas. Des négatifs d'enlèvements orientés soit dans l'axe de l'outil soit perpendiculairement à celui-ci, sont visibles mais compte tenu des retouches multiples, il est difficile de dire s'il s'agit d'une préparation spécifique du tranchant ou s'ils ne résultent que des phases antérieures de débitage. Un véritable « coup de tranchet » n'est présent que sur un seul outil. Les outils ont une forme rectangulaire à trapézoïdale, et dans deux cas une forme triangulaire. Les supports recherchés pour le façonnage des tranchets sont plutôt grands et épais (fig. 29 et 31). Un seul outil s'individualise par sa petite taille (fig. $32, n^{\circ} 6$ ), qui le place aux limites de sa fonctionnalité. 

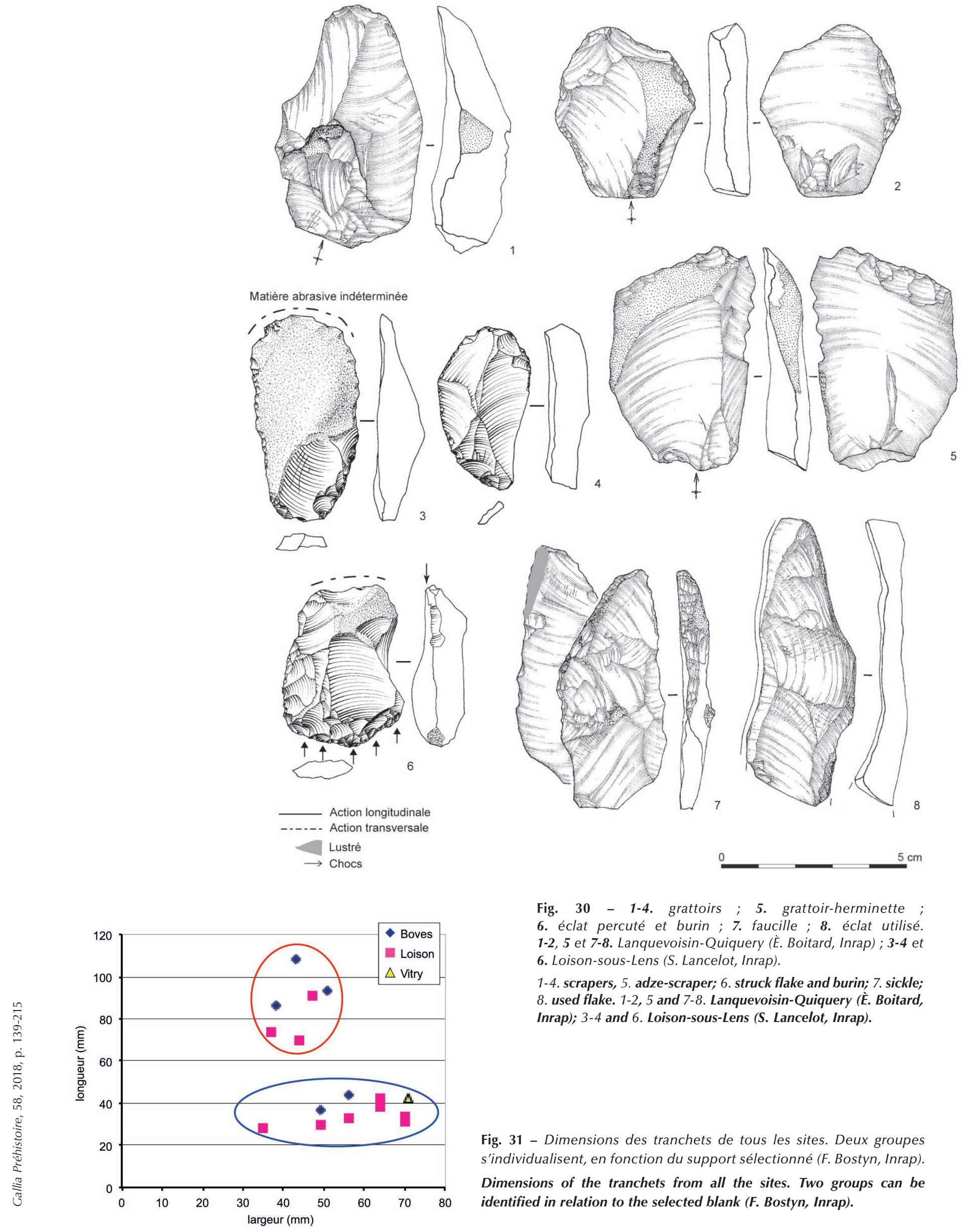

Fig. 30 - 1-4. grattoirs ; 5. grattoir-herminette ; 6. éclat percuté et burin ; 7. faucille; 8 . éclat utilisé. 1-2, 5 et 7-8. Lanquevoisin-Quiquery (̇̇. Boitard, Inrap) ; 3-4 et 6. Loison-sous-Lens (S. Lancelot, Inrap).

1-4. scrapers, 5. adze-scraper; 6. struck flake and burin; 7. sickle; 8. used flake. 1-2, 5 and 7-8. Lanquevoisin-Quiquery (Ė. Boitard, Inrap); 3-4 and 6. Loison-sous-Lens (S. Lancelot, Inrap).

Fig. 31 - Dimensions des tranchets de tous les sites. Deux groupes s'individualisent, en fonction du support sélectionné (F. Bostyn, Inrap). Dimensions of the tranchets from all the sites. Two groups can be identified in relation to the selected blank (F. Bostyn, Inrap). 

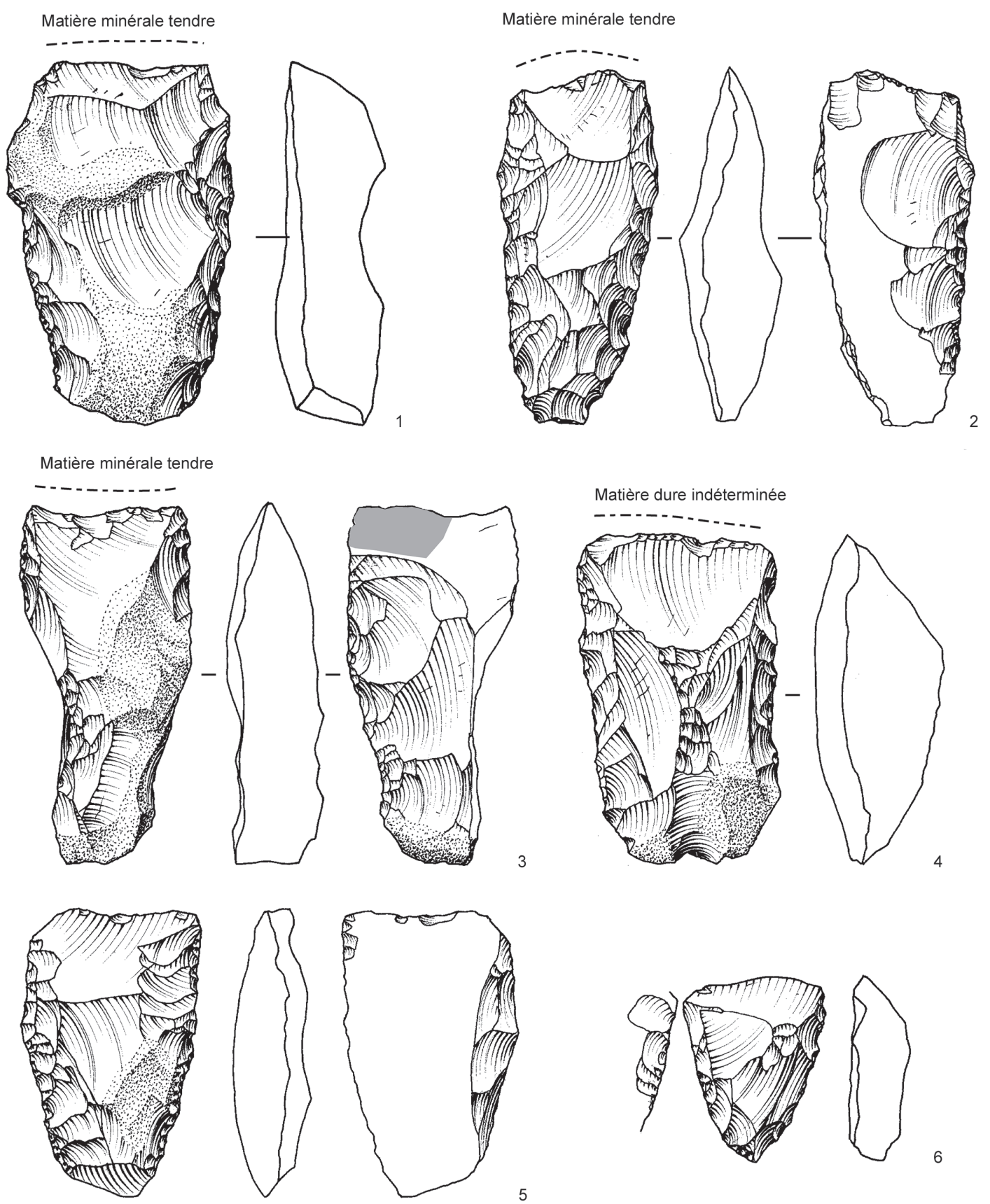

5
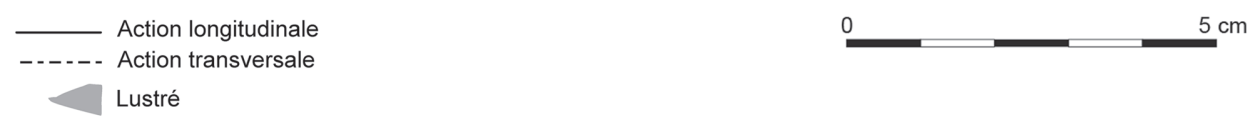

Fig. 32 - Tranchets (Loison-sous-Lens ; S. Lancelot, Inrap).

Tranchets (Loison-sous-Lens; S. Lancelot, Inrap).

$\mathrm{Au}$ sein des assemblages, on remarque que certains supports ont été utilisés bruts sans retouches préalables : ces éclats utilisés ne présentent aucun caractère commun et ont été sélectionnés sans doute en fonction du besoin ponctuel. Dans le même ordre d'idée, deux pièces présentent un émoussé prononcé à une extrémité et une autre a été utilisée en percussion lancée modérée sur un bord.
Les autres catégories d'outils sont représentées plus ponctuellement, par un à cinq outils. On soulignera la présence de deux éléments de faucille, éclats présentant le lustré brillant disposé en oblique par rapport au bord du support, ce qui est rare, ce type d'outil étant en général façonné sur lame.

La série d'outils sur lame comprend 81 outils répartis de manière toujours inéquitable entre les sites (tabl. 5). La diversité 


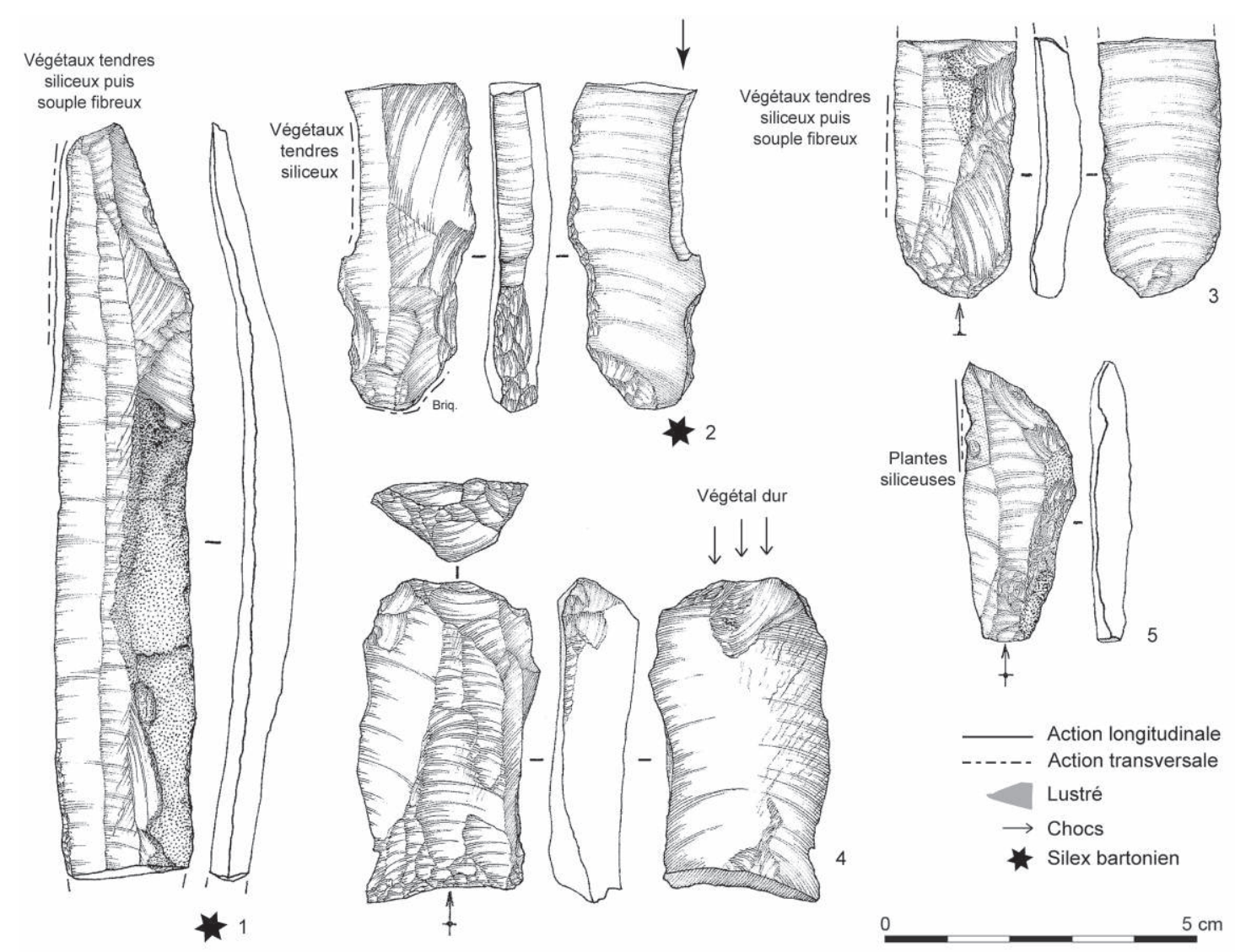

Fig. 33 - 1 et 3. lames utilisées ; 2. burin sur lame; 4. grattoir-herminette ; 5. lame de faucille (Lanquevoisin-Quiquery;

1 and 3. used blades; 2. burin on blade; 4. adze-scraper; 5. sickle blade (Lanquevoisin-Quiquery; È. Boitard, Inrap).

des matières premières est nettement plus grande qu'au sein de l'outillage sur éclat, et, de surcroît concerne des matériaux différents. Le silex bartonien avec vingt-six outils (soit 32,1 \%) est la seconde matière première sélectionnée pour l'outillage.

Un tiers de la série est représenté par les burins. On remarquera que dans ce cas, c'est le silex bartonien qui a été le plus sélectionné (plus de la moitié des outils). Ils sont indifféremment sur cassure (13), sur troncature (5), dièdres (3) ou associent différents types de mise en forme (troncature / cassure ou dièdre / cassure ou troncature, etc.). Les lames sélectionnées sont des lames de plein débitage choisies pour leur longueur que l'on devine plutôt grande sur les outils peu transformés (fig. $25, \mathrm{n}^{\circ} 1$ et fig. $33, \mathrm{n}^{\circ} 2$ ), pour leur largeur supérieure à $20 \mathrm{~mm}$ et surtout pour leur épaisseur supérieure à $4 \mathrm{~mm}$. L'un des outils présente un émoussé très prononcé sur la partie proximale opposée au burin (fig. $33, \mathrm{n}^{\circ} 2$ ). Un lustré brillant a été observé sur le pan de cinq outils (fig. $25, \mathrm{n}^{\circ} 1$ et 3 ; fig. $33, n^{\circ} 1-2$ ) alors que six autres ont des bords avec une fine retouche marginale.

La seconde catégorie d'outil est composée de quatorze lames de faucille, et ce sur les deux sites les plus riches, ce qui est assez exceptionnel sur les sites BVSG. Ajouté à cela que sur les deux mêmes sites, des éclats ont été aussi utilisés pour cette fonction (fig. $30, \mathrm{n}^{\circ} 7$ ), on comprend que le travail de récolte a été important au sein des activités artisanales.
Les outils sont mis à gabarit par une troncature oblique rectiligne ou légèrement convexe (fig. $34, n^{\circ} 2-3$ et 7 ), le lustré caractéristique se situant sur le bord opposé. Dans un cas, la retouche abrupte constitue un dos continu (fig. $33, \mathrm{n}^{\circ} 5$ ), un autre outil a été tronqué à la base et un unique exemple a été utilisé brut (fig. $34, \mathrm{n}^{\circ} 8$ ).

Les lames retouchées et utilisées arrivent ensuite avec un effectif respectif de douze et neuf. Elles présentent un taux élevé de fracturation puisque seuls trois outils sont entiers. Les retouches sont toujours marginales, irrégulières et souvent discontinues. Les lames utilisées présentent, quant à elles, un lustré brillant sur un bord ou un émoussé léger ou encore des retouches très marginales et irrégulières.

Les armatures sont représentées par huit pièces qui proviennent presque toutes de Loison-sous-Lens. Les deux types connus sont représentés de manière égale, soit quatre armatures perçantes et quatre armatures tranchantes (fig. 35). Les flèches perçantes sont de forme triangulaire asymétrique dans trois cas, avec un bord et une base tronqués par retouches directes abruptes. La fracturation des lames par la technique du microburin a été employée dans deux cas. La base est légèrement concave sur deux des pièces. D'un point de vue dimensionnel, ces dernières sont très calibrées et ont des longueurs comprises entre 20 et $30 \mathrm{~mm}$ pour des largeurs variant de 14 à $20 \mathrm{~mm}$. Les armatures tranchantes sont façonnées par double tronca- 

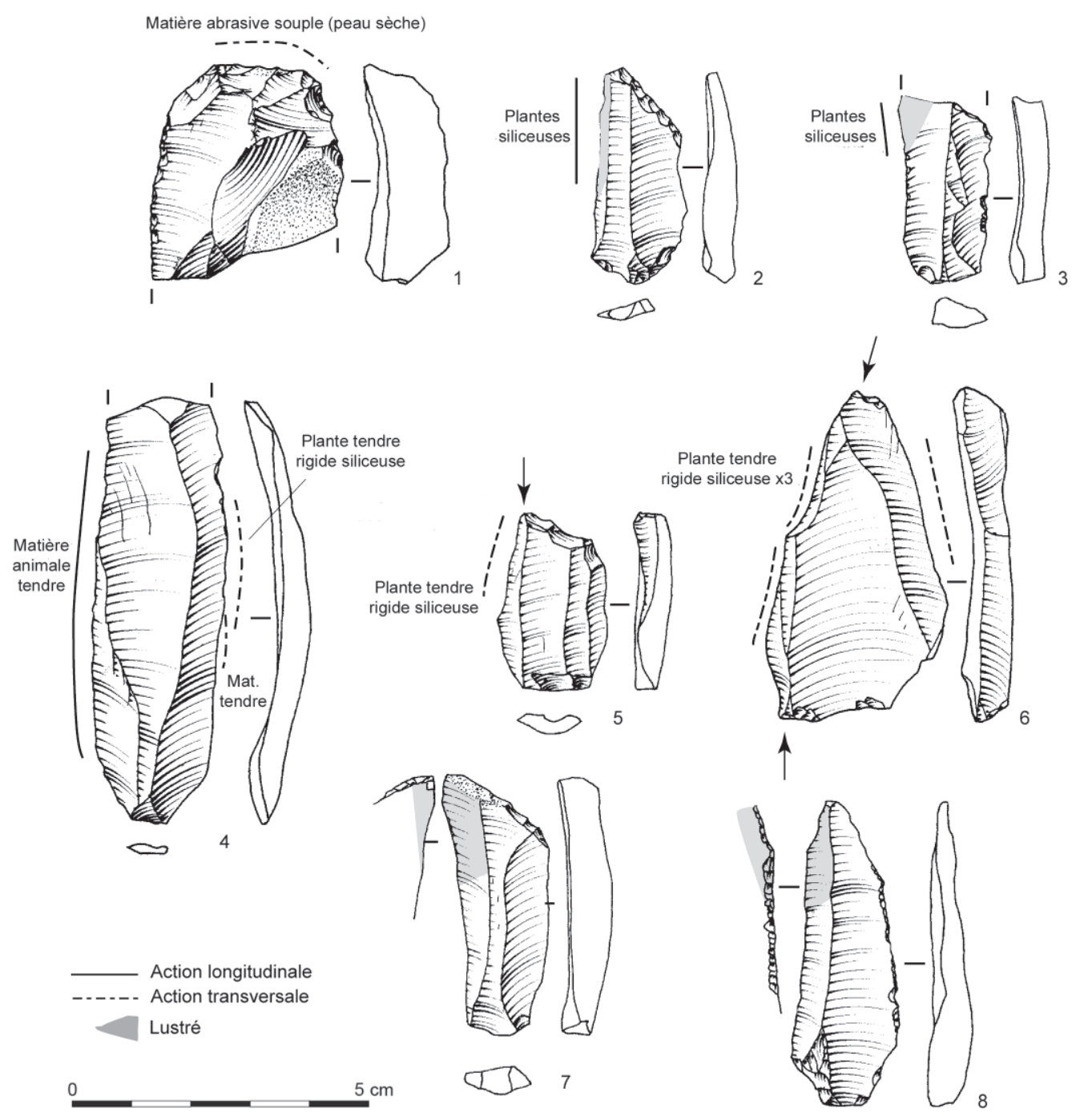

Fig. 34 - 1. grattoir ; 2-3 et 7-8. lames de faucille ; 4. lame utilisée ; 5-6. burins sur lame (Loison-sous-Lens ; S. Lancelot, Inrap). 1. end scraper; 2-3 and 7-8. sickle blades; 4. used blade; 5-6. burins on blades (Loison-sous-Lens; S. Lancelot, Inrap).
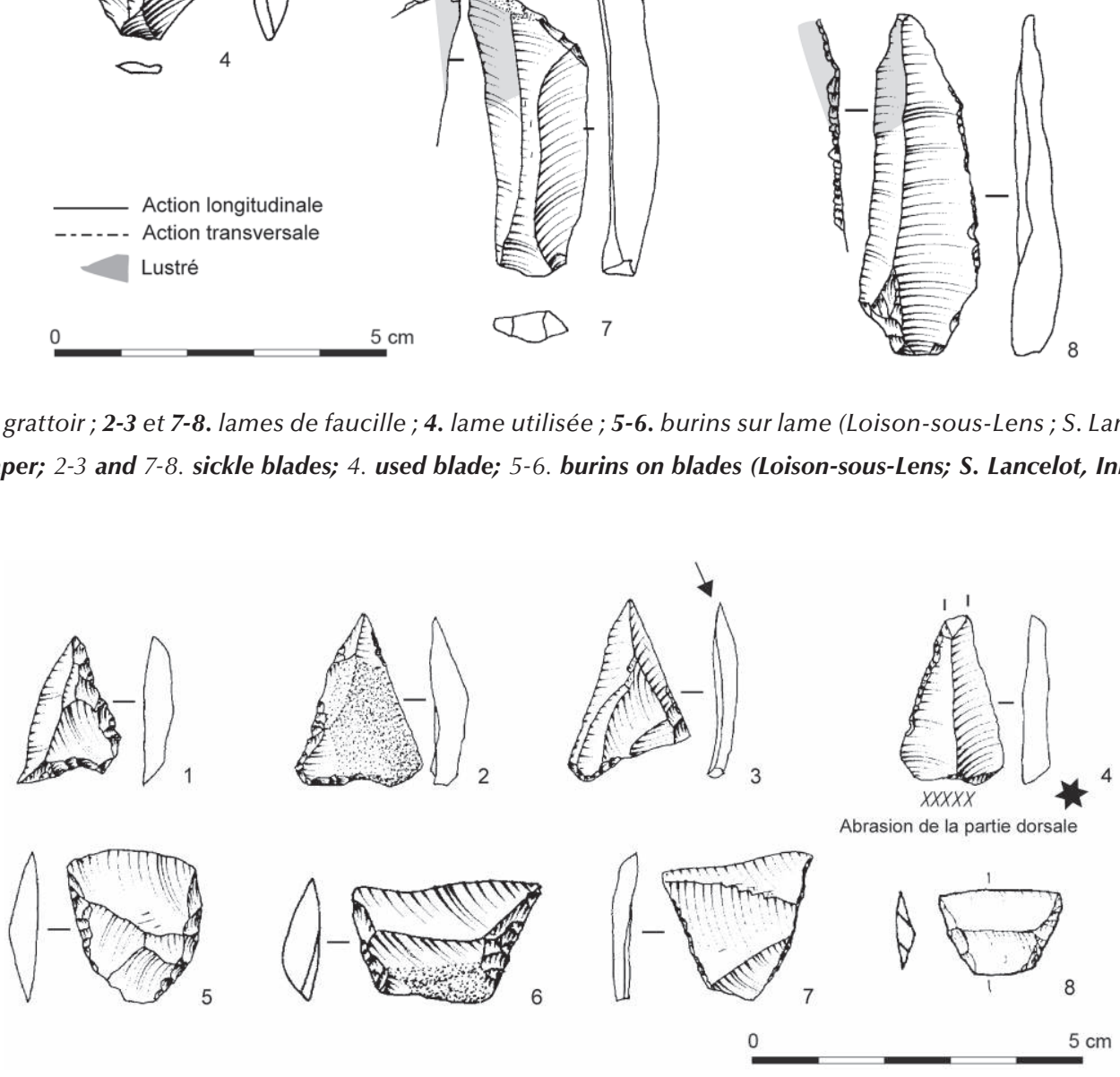

Fig. 35 - Armatures de flèches perçantes (1-4) et tranchantes (5-8). 1-7. Loison-sous-Lens, 8. Vermand (S. Lancelot, Inrap ; sauf 8 : F. Bostyn, Inrap).

Perforating arrowheads (1-4) and cutting arrowheads (5-8).

1-7. Loison-sous-Lens; 8. Vermand (S. Lancelot, Inrap; except 8: F. Bostyn, Inrap). 
ture rectiligne en oblique par rapport à l'axe de débitage, leur donnant une forme trapézoïdale. Une seule armature est en silex tertiaire, il s'agit d'une perçante (fig. $35, n^{\circ} 4$ ). La seule armature provenant du site de Vermand, par sa petite taille, pourrait rappeler les modèles mésolithiques bien que les trapèzes mésolithiques soient plus dissymétriques (Ducrocq 2001).

Parmi les autres types d'outils représentés par quelques exemplaires, on note la présence de troncatures, qui pourraient être des ébauches de lames de faucille non terminées ou non utilisées. Deux perçoirs ont été retrouvés à Loison-sous-Lens et Languevoisin-Quiquery. Tous les deux ont été réalisés sur une lame en silex bartonien et la partie active a été dégagée par de la retouche directe, abrupte et convergente des deux bords (fig. 25, $\mathrm{n}^{\circ}$ 15-16). Terminons cet aperçu de l'outillage sur lame en soulignant la quasi-absence de grattoir puisqu'un unique outil de ce type a été trouvé à Loison-sous-Lens.

\section{LES REGISTRES D'ACTIVITÉ IMPLIQUANT L'INDUSTRIE LITHIQUE À LoIsON-SOUS-Lens et à LangueVoisin-QUiQuerY}

\section{Données générales}

50 des 100 outils analysés à Loison-sous-Lens et 36 des 58 à Languevoisin-Quiquery ont livré des traces d'utilisation (respectivement 57 et 62 zones d'utilisations ou ZU au total). Ces résultats sont cohérents avec ce qui est habituellement enregistré en contexte domestique. Des petites divergences apparaissent dans la gestion et l'intensité d'utilisation des deux séries. À Languevoisin-Quiquery, les pièces à utilisations multiples sont nombreuses (18 des 36 pièces avec traces) et elles portent jusqu'à $5 \mathrm{ZU}(17$ à $1 \mathrm{ZU}, 13$ à 2 et 1 à 3,3 à 4,1 à $5 \mathrm{ZU})$. À Loison-sousLens, ces pièces sont plus rares ( 5 des 50 pièces avec traces) et elles ne portent pas plus de trois zones usées.

Que ce soit à Languevoisin-Quiquery ou à Loison-sous-Lens, les résultats tracéologiques révèlent des spectres d'activités relativement diversifiées (tabl. 6). Ces dernières concernent tant la subsistance que l'artisanat. Le nombre d'outils en silex relatif à chacune des opérations détectées est très variable. Certaines activités n'ont nécessité qu'une catégorie typologique d'outil : le travail de la terre avec les tranchets, la moisson avec des petits inserts sur lame relativement standardisés, le grattage des peaux avec les grattoirs. D'autres activités ont requis un panel d'outils plus important : le raclage des plantes tendres siliceuses fraîches ou fibreuses sèches avec les burins, des lames brutes ou retouchées, des éclats retouchés, le raclage du bois avec les denticulés, les éclats retouchés. À Languevoisin-Quiquery, les relations typo-fonctionnelles semblent de manière générale moins strictes entre catégories d'outils et activité qu'à Loison-sous-Lens.

À Languevoisin-Quiquery, 13 des 17 outils sur lame en silex tertiaire analysés portent des zones d'utilisation souvent multiples (6 d'entre eux et jusqu'à $5 \mathrm{ZU}$ par pièce). Les matériaux mis en œuvre les plus fréquents sont les plantes tendres à l'état frais (17 ZU) ou sec (6 ZU) et dans une moindre mesure de la peau ( $3 \mathrm{ZU}$ ). Sur les 13 outils sur lame en silex secondaire analysés, six portent des zones d'utilisation. Pour quatre d'entre eux, ces zones d'utilisation sont multiples (jusqu'à 3 ZU). Concernant les matières mises en œuvre, les plantes tendres à l'état frais restent les plus fréquemment diagnostiquées (5 ZU) devant les plantes fibreuses souples et sèches $(2 \mathrm{ZU})$. Les deux industries laminaires partagent ainsi des points communs avec une forte implication dans le travail des plantes à la nuance près que le silex tertiaire présente plus favorablement des zones usées.

Avec deux outils en silex tertiaire analysés portant chacun une unique zone d'utilisation, les données sont trop faibles à Loison-sous-Lens pour appréhender les différences de gestion entre l'utilisation du silex tertiaire et l'utilisation du silex secondaire. Les outils sur lame, toutes catégories confondues, sont très impliqués dans le travail des plantes non ligneuses (13 ZU) devant les matières animales tendres ( $2 \mathrm{ZU})$, le bois $(1 \mathrm{ZU})$ et les matières minérales $(1 \mathrm{ZU})$.

\section{Les activités liées à l'acquisition}

La récolte des plantes tendres siliceuses souples est attestée par la présence de petites armatures lustrées aménagées sur lame. Cinq armatures de faucille ont été analysées à Loison-sous-Lens (fig. 34, $n^{\circ}$ 2-3) et quatre à Languevoisin-Quiquery (fig. 33, $\mathrm{n}^{\circ}$ 5). Les modalités d'utilisation et les caractéristiques de l'emmanchement répondent au standard danubien : les lames sont insérées souvent de biais dans un manche les unes à la suite des autres. La probabilité que l'usure observée sur ces armatures corresponde à la moisson des céréales est forte mais notre champ de comparaison expérimental ne recouvre pas encore tout le panel de plantes (les graminées) qui ont pu être récoltées. La carpologie et les nombreux éléments de mouture, à Loison-sous-Lens, renforcent néanmoins cette probabilité. Toutes les armatures reconnues portent un lustré très prononcé, correspondant à une utilisation durant de nombreuses heures (fig. $36, \mathrm{n}^{\circ} 2$ ).

Seul le site de Loison-sous-Lens a livré des armatures de flèche. Parmi elles, seules une armature tranchante et une armature perçante portent des fractures et des enlèvements de morphologie complexe susceptibles d'avoir été laissés par des impacts violents, en lien avec une fonction de projectile. Une dernière armature perçante porte des traces d'une abrasion intentionnelle de sa base sur une matière minérale ou de la peau sèche sale. Ce procédé « de meulage » (abrasion intentionnelle des bords) est habituellement observé sur les armatures danubiennes (Caspar 1988, Vaughan 1994). Ces stigmates sont souvent interprétés comme un procédé destiné à éviter la rupture des liens de fixation ou à renforcer la résistance de la pointe apicale lors de l'impact.

Abréviations utilisées dans le tableau 6

$\begin{array}{llll}\mathbf{M A T} & \text { Matière animale tendre } & \mathbf{M M T} & \text { Matière minérale tendre } \\ \mathbf{M D A} & \text { Matière dure animale } & \mathbf{M D I} & \text { Matière dure indéterminée } \\ \mathbf{M V S} & \text { Matière végétale siliceuse } & \mathbf{M T I} & \text { Matière tendre indéterminée } \\ \mathbf{M V S F} & \text { Matière végétale fibreuse sèche } & \mathbf{M I} & \text { Matière indéterminée } \\ \mathbf{M V D} & \text { Matière végétale dure } & \mathbf{P e r f o} . & \text { Perforation } \\ \mathbf{M V T R} & \text { Matière végétale tendre rigide } & \mathbf{P e r c u} & \text { Percussion } \\ \mathbf{M M} & \text { Matière minérale } & \mathbf{Z U} & \text { Zone usée }\end{array}$




\begin{tabular}{|c|c|c|c|c|c|c|c|c|c|c|c|c|c|c|c|}
\hline$\sum \vec{z}$ & $F$ & & ๑) & - & 0 & & $\nabla$ & ம & - & $-\sim$ & - & & $m$ o & & - i \\
\hline 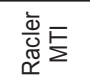 & & & & - & & & & & & & & & & & - \\
\hline 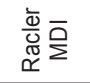 & - & & & & - & & & & & & & & & m & ما \\
\hline 誩高 & & & & & & & & & & 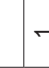 & & & & & - \\
\hline 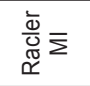 & - & & & & & & & $\sim$ & & & & & & & m \\
\hline 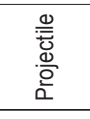 & & & & & & & & & & & & & $\sim$ & & N \\
\hline 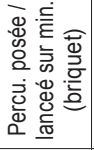 & & & & & & & & & & & & & & & - \\
\hline 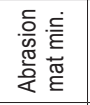 & & & & & & & & & & & & & - & & - \\
\hline 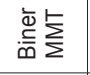 & & & & & & & & & & & & & 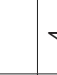 & $\sigma$ & ナ \\
\hline 这㙂 & & & & & & & & & & & - & & & & - \\
\hline 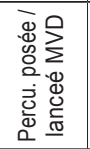 & & & & & & & & & & & & & 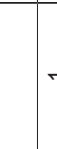 & - & - \\
\hline $\begin{array}{l}\text { 离o } \\
\text { 言产 }\end{array}$ & - & & & & & & & & & & & & & & - \\
\hline 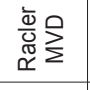 & & & & & مـ & & & - & & $\tau$ & & & & & 0 \\
\hline 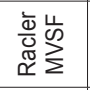 & & & & - & & & - & & & & & & & & $\sim$ \\
\hline 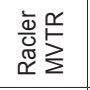 & & & & 6 & & & & & & - & & & & & r \\
\hline 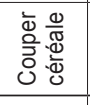 & & & ద & & & & & & & & & & & & مـ \\
\hline 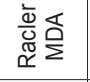 & & & & & & & & $\sim$ & & & & & & & $\sim$ \\
\hline 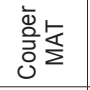 & & & & & & & $m$ & & & & & & & & m \\
\hline 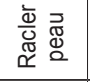 & $\infty$ & & & & & & & & & & & & & & $\infty$ \\
\hline 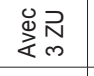 & & & & - & & & - & & & & & & & & $\sim$ \\
\hline 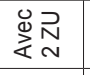 & & & & & $\sim$ & & & & & - & & & & & m \\
\hline 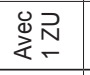 & $F$ & 0 & ๑) & + & $\sim \sim$ & 0 & $\sim$ & 0 & 0. & -0 & - & 0 & $\infty 0$ & $\infty$. & -8 \\
\hline 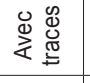 & $F$ & 0 & ه & . & 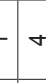 & 0 & $m$ & 0 & 0. & - & & 0 & $m a$ & $\infty$ & - in \\
\hline 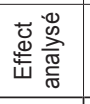 & \pm & - & $\infty$ & $s$ & $+\circ$ & o & o & 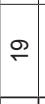 & - & -- & & $m$ & $0:$ & & $-\circ$ \\
\hline $\begin{array}{l}\frac{\bar{o}}{.0} \\
\frac{.0}{9} \\
\dot{\alpha} \\
\dot{\alpha}\end{array}$ & . & 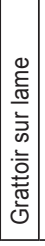 & 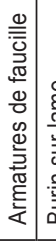 & 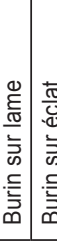 & 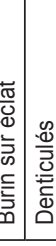 & 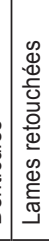 & 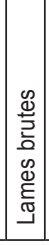 & 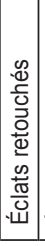 & 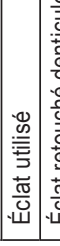 & 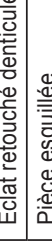 & & 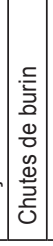 & 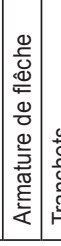 & 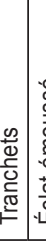 & 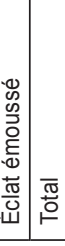 \\
\hline
\end{tabular}

\begin{tabular}{|c|c|c|c|c|c|c|c|c|c|c|c|}
\hline$\frac{m}{z} \vec{N}$ & $\sim$ & 2 & $\diamond$ & $\infty$ & 10 & $\sim$ & $=$ & $\diamond 0$ & & - & $\sim \Im$ \\
\hline 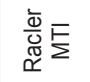 & & & & ऽ & & & m & o & & & $-F$ \\
\hline$\frac{\dot{g}}{\overline{0}} \bar{\Sigma}$ & & & & & & & & & . & & . \\
\hline 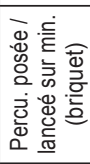 & & & & - & & & & & & & \\
\hline 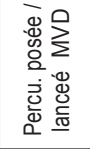 & $\sim$ & & & & & & & & & & o \\
\hline 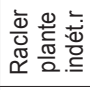 & & & & - & & & & & & & - \\
\hline 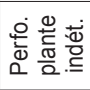 & & & & & & & & & & - & - \\
\hline 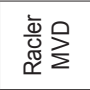 & & & & & $\nabla$ & & & - & & & 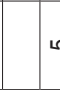 \\
\hline 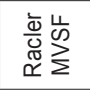 & & & & $\sim-$ & & & $m$ & $-\circ$ & & & $\circ$ \\
\hline $\begin{array}{l}\overline{\bar{o}} \infty \\
\overline{\bar{c}} \\
\mathrm{c}\end{array}$ & & & & - & & & - & & & & ภ \\
\hline 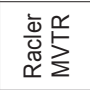 & & & r & $\wedge$ ค & - & & $\nabla$ & $\sim \curvearrowright$ & & & $-\because$ \\
\hline 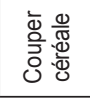 & & & $\sigma$ & & & & & & & & - \\
\hline 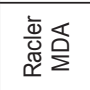 & & & & & & & & . & & & r \\
\hline 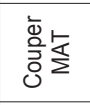 & & & & & & & & . & & & . \\
\hline 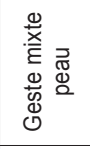 & & & & & & $\sim$ & & & & & o \\
\hline 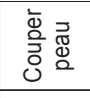 & & & & - & & & & . & & & c \\
\hline 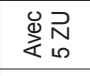 & & & & - & & & & & & & . \\
\hline 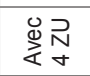 & & & . & - & & & - & & & & o \\
\hline 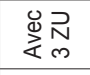 & & & r. & -7 & & & & & & & c \\
\hline $\begin{array}{l}0 \\
\stackrel{0}{<} N \\
\text { N }\end{array}$ & & & & $\sim$ & $\sim$ & - & $m$ & $-\sigma$ & & & $-:$ \\
\hline 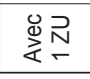 & $\sim$ & & $\nabla$ & $m$ & - & & - & $\sim \Omega$ &. & - & ? \\
\hline 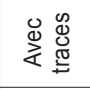 & $\sim$ & 0 & + & $\infty-$ & $m$ & - & $\infty$ & $m$ & & - & $-\therefore$ \\
\hline 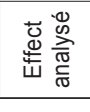 & $\sim$ & $\sim$ & $\diamond$ & 0. & - & - & 웅 & $0:$ & & - & $-i$ \\
\hline 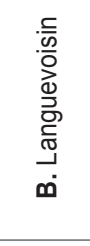 & 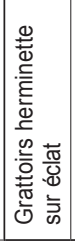 & 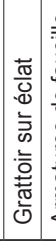 & 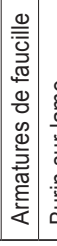 & 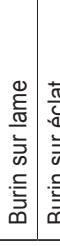 & 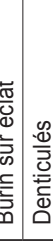 & 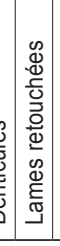 & 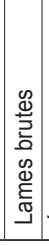 & 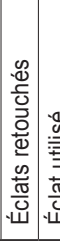 & & 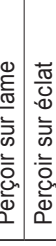 & 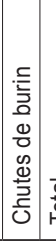 \\
\hline
\end{tabular}


Les opérations de découpe des matières animales pouvant se rattacher à la boucherie mais aussi à diverses autres opérations artisanales (découpe de peaux fraîches, récupération des tendons) sont représentées sur les deux sites. Néanmoins, en raison du faible développement et du caractère souvent ambigu des traces de boucherie, il est fort probable que cette activité soit sous-évaluée au sein de nos séries. Ces opérations ont tout de même été enregistrées à Languevoisin-Quiquery, où une zone active associe une usure laissée à la fois par de brefs contacts avec de l'os et des tissus carnés sur le bord d'un éclat. À Loisonsous-Lens, trois lames brutes portent des stigmates en lien avec la découpe de tissus carnés ou cutanés et ont pu participer tant dans des activités de subsistance que dans des activités liées à l'artisanat, de la peau par exemple (fig. 34, $\mathrm{n}^{\circ} 4$ ).

\section{Les activités de transformation}

Le travail de la peau est bien représenté à Loison-sousLens par la présence d'usures de raclage sur les fronts de huit grattoirs sur éclat (fig. 34, no 1). L'intensité des usures ainsi que la composante abrasive ne sont jamais prononcées et d'après les caractéristiques des polis et des émoussés, il semble que les peaux travaillées aient toujours gardé un certain degré d'humidité. Dans le processus de transformation de la peau en cuir, ces grattoirs pourraient avoir été utilisés après tannage à plusieurs étapes du corroyage, pour affaiblir, amincir ou encore pour l'assouplissement des peaux. En amont du tannage, ces grattoirs ont également pu participer à diverses étapes telles l'effleurage préliminaire, afin de favoriser la pénétration de substances tannantes. À Languevoisin-Quiquery, aucun grattoir ou aucun autre outil ne témoignent de ces activités de raclage, fait extrêmement rare pour un site de cette période où le travail des peaux est toujours bien représenté. Une lame retouchée porte des stigmates d'une action indéterminée (raclage et découpe ?) sur de la peau à l'aide de ses deux bords latéraux. Un burin sur lame et un éclat utilisé ont également été actionnés avec une matière abrasive souple probablement de type cutané.

Les activités en lien avec l'acquisition ou la transformation $\mathrm{du}$ bois sont représentées sur les deux sites. Deux grattoirs herminettes (fig. 33, n 4) à Languevoisin-Quiquery témoignent de contacts en percussion lancée à l'aide de leurs fronts sur du bois, outil qui semble se généraliser dès la fin du Rubané final du bassin de la Seine et est fréquent durant tout le BlicquyVilleneuve-Saint-Germain (Caspar et Burnez-Lanotte 1996, Allard et al. 2004). Un unique grattoir sur éclat provenant de Loison-sous-Lens semble avoir découpé du bois à l'aide de son bord latéral brut. Un tranchet a été actionné en percussion lancée ou posée sur du bois. Enfin, une pièce esquillée de ce même site a probablement raclé un végétal ligneux. Sur les quatre denticulés de Loison-sous-Lens et les trois de Languevoisin-Quiquery portant des traces, ces dernières sont limitées à l'extrémité des dents et ne couvrent pas les coches. Les enlèvements d'usage sont rares et le poli est toujours faiblement développé. Il se présente parfois sous la forme de véritables plages de poli (d'extension modérée et de trame serrée à unie, brillant, plat à dur bombé) ou plus souvent sous la forme de spots de poli couvrant essentiellement la topographie haute du silex et de coulures sur les arêtes du bord et des rares enlèvements d'usage. Ce cortège de traces semble indiquer une utilisation brève de raclage sur un matériau d'origine végétale ligneuse. Conformément à ce que l'on enregistre sur de nombreux sites BVSG, l'acquisition et la transformation du bois sont assez bien représentées sur les bords des outils en silex. Plus que pour du façonnage par raclage d'objet en bois (les expérimentations montrent que ces outils ne sont pas les plus efficaces), ces denticulés pourraient intervenir dans des travaux de faible ampleur, pour la fabrication de lanières en écorce fibreuse par exemple (Caspar et Burnez-Lanotte 2008). Il s'agit du mode d'utilisation le plus fréquent au Rubané et Blicquy-Villeneuve-Saint-Germain (Caspar 1988, Giligny et al. 2001).

Diverses opérations relevant de la transformation des végétaux tendres par raclage sont bien attestées sur les deux sites. Trois principaux types de stigmates correspondant à ces activités ont fréquemment été relevés sur les bords des outils des deux séries. Ces usures relèvent pour la plupart des problématiques du «poli $23 »$ (Caspar et al. 2005).

- Dans certains cas, l'usure se manifeste sous la forme d'un lustre marginal brillant ondulé organisé en bande le long du fil actif (fig. 34, $\mathrm{n}^{\circ} 4$ ). À Loison-sous-Lens, cette trace concerne trois burins sur lame (fig. 34, no 5-6) et un éclat retouché (7 ZU). À Languevoisin-Quiquery, elle concerne trois burins sur lame (fig. 33, $n^{\circ} 2$ ) et un burin sur éclat $(10 \mathrm{ZU})$. Les bords actifs concernés sont les pans des burins ou les bords bruts, d'angulation ouverte à délinéation rectiligne ou légèrement concave. Les zones usées sont comprises entre 10 et $40 \mathrm{~mm}$. Bien que non reproduit expérimentalement, les similitudes les plus pertinentes pour cette usure s'établissent avec polis expérimentaux utilisés en coupe transversale posée sur des plantes tendres rigides, probablement de type roseau ou phragmite. B. Gassin a observé des concordances entre traces expérimentales et archéologiques lors du raclage de cannes de Provence, notamment pour la préparation des hampes de flèche (Gassin 1996).

- Plusieurs outils de Languevoisin-Quiquery portent une usure à double aspect caractérisé par l'association de polis transversaux de type distinct sur les faces adjacentes d'une même portion de bord ( $9 \mathrm{ZU})$. Cette usure concerne deux burins sur lame, trois lames brutes (fig. $33, n^{\circ} 1$ et 3 ), un burin sur éclat, un éclat retouché et un éclat utilisé. Les angles de taillant des bords sont ouverts. Sur une face, préférentiellement l'avers toutes catégories typologiques confondues, on retrouve l'usure précédemment décrite, la trace brillante de type additif et d'aspect ondulé. Sur la face opposée du même bord, on observe une usure abrasive mate de type cutané, chargée de strie (fig. 36, $\mathrm{n}^{\mathrm{o}} 3$ ). L'interprétation problématique de ce cortège de traces a fait l'objet de multiples publications et synthèses entre partisans d'une usure occasionnée par une seule et même activité indéterminée (Beugnier 2000, Beugnier et Crombé 2007, Van Gijn 2010) et partisans d'une formation diachronique de cette usure, précisément en deux étapes 


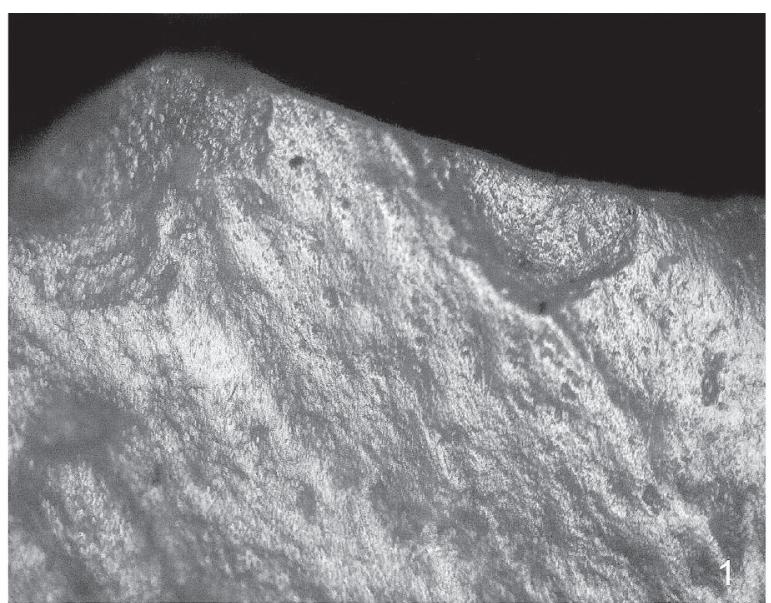

Poli 50 (revers) : détail de la zone usée par de la percussion posée/lancée transversale sur une matière minérale meuble $(x$ 100)

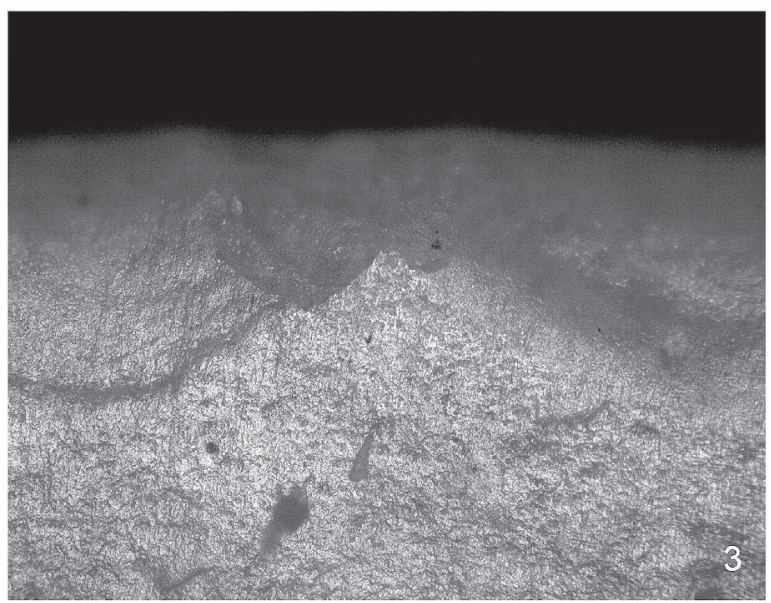

Poli 25 (revers) : face de contact de la zone usée par le raclage d'une matière abrasive souple. probablement des fibres véqétales souples et sèches $(x 100)$

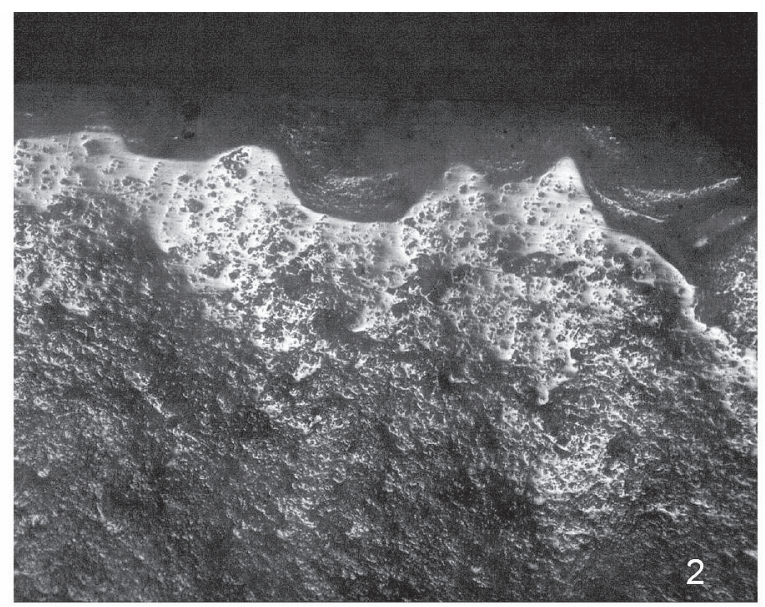

Poli 21 (revers) : détail de la zone usée par la coupe d'une matière végétale tendre siliceuse ( $\times 50)$

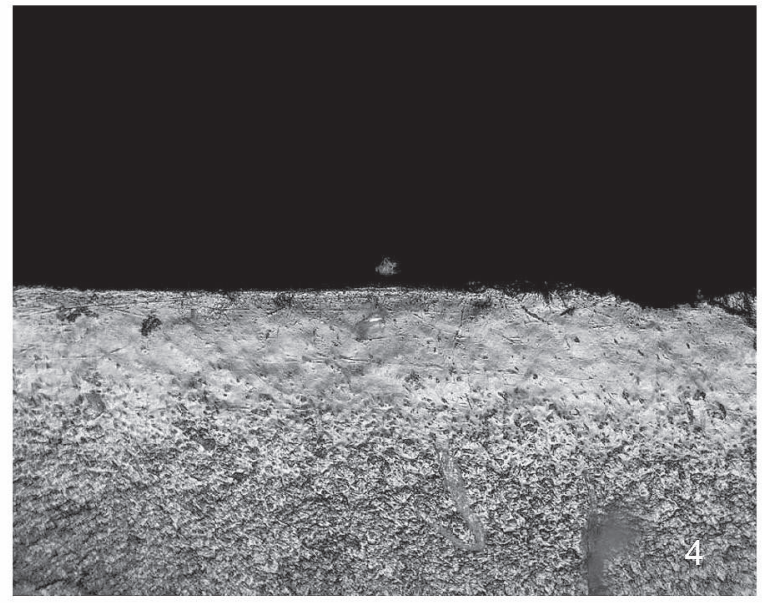

Poli 23 (avers) : face d'attaque d'un burin ayant raclé une matière véqétale tendre siliceuse. probablement rigide (x 100)

Fig. 36 - Vue microscopique de différents polis d'usure. 1. poli 50, 2. Poli 21, 3. Poli 25 ; 4. Poli 23 (N. Cayol, Inrap). Microscopic view of the different types of use-wear polish. 1. polish 50; 2. polish 21; 3. polish 25; 4 . polish 23 (N. Cayol, Inrap).

(Caspar et al. 2005 et 2007, Caspar et Burnez-Lanotte 2008). Cette hypothèse nous semble la plus pertinente. Dans un premier temps, une activité de raclage de plantes siliceuses tendres génère le micro-dépôt brillant et ondulé. Comme nous l'avons décrit plus haut, cette usure est présente sous sa forme simple sur plusieurs outils des deux sites. Dans un second temps, une activité de teillage de fibres végétales souples et sèches produit l'usure abrasive de type cutané. L'intérêt de recycler un bord lustré pour le teillage permet de gagner en efficacité, en augmentant la production de filasse et en limitant la production d'étoupe. Le teillage, opération visant à séparer les fibres libériennes, après rouissage et séchage, des parties ligneuses de plantes tel le lin ou le chanvre, illustre la présence sur ces deux sites d'activités de préparation et de transformation d'éléments en lien avec le textile et les fibres végétales (vannerie, filage, corderie et sparterie...).

- L'usure laissée par le teillage de fibres végétales souples et sèches sous sa forme simple (non associée au raclage de plantes à l'état frais) a pu aussi être diagnostiquée à Loisonsous-Lens sur le bord brut d'un burin sur éclat et, bien que l'usure soit plus douteuse, sur le bord d'une lame brute.

À l'inverse d'autres activités comme la découpe de tissus carnés, les usures résultant du raclage de ces matériaux, sont souvent bien développées et plus facilement détectables, ce qui pourrait expliquer en partie leur meilleure représentation au sein des cortèges des traces repérées.

À Loison-sous-Lens, quatre tranchets ont été actionnés en percussion lancée ou posée sur une matière minérale meuble, probablement à la manière d'une houe ou d'une herminette (fig. $32, \mathrm{n}^{\circ} 1$ à 3 ).

Les bords sont fortement endommagés et les stigmates sont relativement bien marqués et envahissants. La trace sur les tranchants actifs est envahissante (jusqu'à $3 \mathrm{~cm}$ ), d'aspect doux luisant à l'œil nu. Au microscope, le poli est de type abrasif mat, à texture grenue à criblée, souligné de nombreuses stries transversales (fig. 36, $\mathrm{n}^{\circ}$ 1). Les opérations dans lesquelles 
auraient pu intervenir ces pièces sont plurielles et tout un champ expérimental reste à explorer pour affiner l'interprétation fonctionnelle de ces pièces. On pense d'ores et déjà à la mise en valeur des sols agricoles, mais ces pièces auraient également pu participer à l'assainissement de zones humides, au terrassement, au travail de l'argile en vue de la confection du torchis (Allard et al. 2004, Philibert 2005).

Les dernières opérations de transformation des matériaux relevées sont plus anecdotiques. À Loison-sous-Lens, on note deux éclats retouchés ayant raclé de l'os et un dernier éclat retouché ayant été actionné en percussion transversale sur une matière minérale, peut-être en tant que briquet. Un perçoir sur lame semble avoir foré une matière minérale tendre, probablement à l'aide d'un archet. À LanguevoisinQuiquery, un éclat porte des traces de contacts transversaux avec une matière dure animale et on note un burin utilisé en briquet. Le reste des zones usées ne peuvent pas être reliées à des matériaux précis (10 ZU à Loison-sous-Lens et $13 \mathrm{ZU}$ à Languevoisin-Quiquery).

\section{Éléments de discussion sur les spectres d'activités détectées}

La plupart des activités détectées à LanguevoisinQuiquery et à Loison-sous-Lens sont celles habituellement reconnues dans les fosses latérales d'unités d'habitation de sites du Blicquy-Villeneuve-Saint-Germain (Bostyn et Cayol 2012, Philibert 1996). Ces résultats témoignent de la forte homogénéité du rôle fonctionnel des industries en silex à cette période. Quelques variations dans leurs représentations sont néanmoins perceptibles. À Loison-sous-Lens, le spectre fonctionnel apparaît bien diversifié et assez équilibré en termes d'activités tournées vers l'acquisition (moisson, chasse) ou les activités artisanales (plantes). Les trois grandes sphères de matériaux animales, végétales et minérales sont toutes représentées par les traces présentes sur les bords des outils (tabl. 6). Néanmoins, par rapport au spectre fonctionnel classique des sites de vallées, on retiendra la moins bonne représentation du travail des peaux.

À Languevoisin-Quiquery, l'économie du site semble beaucoup plus orientée vers la transformation des matières végétales (avec le raclage de plantes fraîches, de fibres végétales et de bois). Les classes typologiques d'outils concernés par ces activités sont plus variées que sur les sites de fond de vallée. En revanche, la chasse, la transformation des peaux en cuir et le travail de la terre, souvent balisées par la présence d'armatures de flèche, de grattoirs et de tranchets sur les sites de l'Aisne et de l'Oise, sont mal représentés, voire absents (tabl. 6).

\section{SyNTHÈSE SUR L'INDUSTRIE EN SILEX : Productions spécialisées et réseaux de CirCulation}

La présentation synthétique des différents assemblages lithiques montre donc à la fois des éléments de convergence et des points de divergences entre les assemblages, mais aussi plus globalement au sein de la culture BVSG. Au vu des effectifs et de l'état de la documentation des différents sites pris en compte, les deux sites de Loison-sous-Lens et Languevoisin-Quiquery serviront de base à la discussion. Les autres seront utilisés autant que faire se peut pour compléter les observations.

Parmi les fondamentaux de l'industrie en silex BVSG (Bostyn 1994, Allard et Bostyn 2006, Denis 2014a), on retrouve la dualité production d'éclat / production de lame sur les matières premières locales sur l'ensemble des sites étudiés, sachant que la première est largement dominante, ce qui différencie bien le BVSG du Rubané (Allard 2005). Les schémas opératoires s'avèrent plutôt simples, bien que la comparaison entre supports bruts et retouchés témoigne de la recherche d'une certaine longueur pour la fabrication de l'outillage. Par contre, l'existence d'une chaîne opératoire spécifique pour la production d'éclats larges pour la fabrication des tranchets reste posée, aucun indice probant ne permettant de militer pour cela. La production laminaire est surtout bien documentée à Loison-sous-Lens et l'ensemble des observations d'ordre technique ou sur les étapes de la chaîne opératoire est strictement comparable à ce qui a pu être décrit sur l'ensemble du Bassin parisien (Bostyn 1994, Allard 1999, Augereau 2004, Denis 2008), témoignant d'une unité technique et, par extension, culturelle sur l'ensemble de la zone géographique occupée par le BVSG. Cependant, les nombreuses études sur les industries lithiques de cette culture (Bostyn 1994, Allard et Bostyn 2006, Denis 2008) ont montré une diminution progressive de la part de la production laminaire au cours du temps, les phases anciennes étant encore très laminaires avec une proportion proche des $20 \%$, alors que dans les phases plus récentes la part laminaire était en forte diminution (fig. 37). Le site de Languevoisin-Quiquery apparaît ici clairement déficitaire en produits laminaires, ce qui constitue une originalité dans le BVSG. Pour la première fois, on observe une gestion des silex crétacés comparable à celle des silex bartoniens avec une circulation de nucléus préformés. On rejoint ici des observations faites en Belgique où les nucléus en silex de Ghlin ont voyagé vers la Hesbaye sous une forme déjà préparée (Denis 2014b). La question de la nature de l'occupation de Languevoisin-Quiquery est ainsi posée au travers des caractéristiques de son industrie lithique.

L'autre facteur d'unité du BVSG bien représenté dans les séries étudiées est la présence du silex bartonien du Bassin parisien. Si la circulation de cette matière première à l'échelle de l'ensemble de la culture BVSG, excepté dans ses marges les plus occidentales (Bostyn et Denis 2016, Charraud 2013, Bostyn et al. à paraître a), est un fait acquis aujourd'hui, les modalités de sa diffusion au sein des villages restent toujours en discussion (Bostyn et Denis 2016). Si certains sites ne reçoivent que des produits laminaires, et c'est le cas ici des sites de Noyelles, Boves, Vermand mais aussi d'Étouvie, la présence de petits éclats non corticaux a été observée sur les sites de Loison-sous-Lens et Languevoisin-Quiquery. Dès lors, la circulation de cette matière première peut s'envisager sous la forme de nucléus préformés, ce qui trouverait un écho sur les sites du Hainaut Belge (Bostyn 2008, Denis 2014a, Bostyn et Denis 2016) sur lesquels, malgré la distance, ces produits sont attestés. Si à Languevoisin-Quiquery, l'hypothèse du 


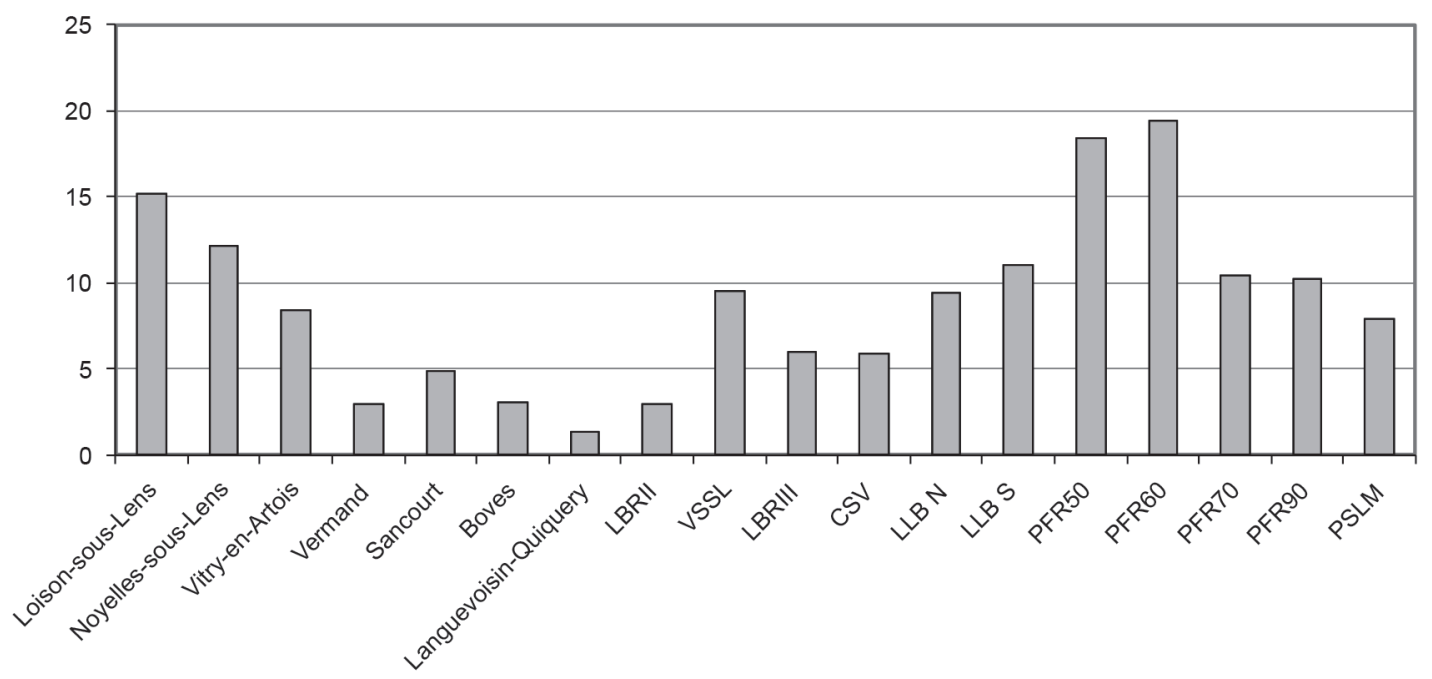

Fig. 37 - Comparaison de la part laminaire dans le silex crétacé des industries de différents sites du BVSG (F. Bostyn, Inrap).

LBRII et III. Longueil-Sainte-Marie « la Butte de Rhuis II et III »; VSSL. Villers-sous-Saint-Leu; CSV. Courcelles-sur-Viosne ; LLB N et S. LongueilSainte-Marie "le Barrage » nord et sud ; PFR. Pontpoint " le Fond de Rambourg »; PSLM. Poses "sur la Mare ».

Comparison of Cretaceous flint blade production at the different BVSG sites (F. Bostyn, Inrap).

LBRII and III: Longueil-Sainte-Marie "Ia Butte de Rhuis II et III"; VSSL: Villers-sous-Saint-Leu; CSV: Courcelles-sur-Viosne; LLB N and S: LongueilSainte-Marie "le Barrage" north and south; PFR: Pontpoint "le Fond de Rambourg"; PSLM: "Poses sur la Mare".

transport des nucléus à lame préformés à la fois en crétacé et en bartonien laisse supposer l'absence de savoir-faire sur place et donc le déplacement du tailleur, le niveau de savoirfaire des tailleurs locaux à Loison-sous-Lens est élevé et ces derniers ont pu procéder à l'exploitation des nucléus en bartonien, les productions étant très similaires. C'est un des éléments importants à souligner : en effet, les produits laminaires en bartonien de Loison-sous-Lens se différencient de ceux du site de Languevoisin-Quiquery par leur caractère plus léger, les largeurs moyennes étant de 19,3 mm contre 21,9 mm à Languevoisin-Quiquery, mais comparables à celles des produits laminaires en crétacé $(18,4 \mathrm{~mm})$.

La présence de silex de Ghlin à Vermand renforce l'idée d'un réseau nord-sud très dynamique puisque, rappelons-le, le silex de Ghlin originaire du bassin de Mons en Belgique, est attesté dans les vallées de l'Oise et de l'Aisne sous la forme exclusive de lames. La Haute vallée de l'Oise relayée par celle de la Somme puis enfin celle de l'Escaut, aurait pu jouer le rôle de voie de passage entre ces deux aires géographiques. L'absence de silex de Ghlin à Loison-sous-Lens en est d'autant plus significative que ce site est géographiquement plus proche des sites du Hainaut que celui de Vermand et a fortiori de ceux des vallées de l'Oise et de l'Aisne. L'axe nord-sud semble être structurant dans la diffusion de certaines productions au BVSG et les habitats du Hainaut ont eu un rôle déterminant dans la circulation des produits et des savoir-faire vers la Hesbaye (Denis 2014b). Ils auraient également pu tenir une place prépondérante dans la redistribution, via des réseaux secondaires, des silex bartoniens vers les sites du Nord de la France. Ces hypothèses devront néanmoins être confortées par des nouvelles découvertes d'habitat dans le Nord de la France.
Si l'on revient sur les compositions des séries d'outils, la première remarque concerne la place des grattoirs au sein des assemblages. En effet, les séries BVSG se caractérisent par la place toujours dominante des grattoirs au sein de l'outillage sur éclat (Bostyn 1994, Allard et Bostyn 2006). Or, aucun des sites concernés par cette étude n'a livré un nombre significatif de grattoirs sur éclat, ceux-ci n'arrivant qu'en troisième position après les denticulés et les éclats retouchés, et en quantité à peine plus élevée que les burins et les tranchets. Par contre, sur les sites du Hainaut, cette faible présence des grattoirs est une constante puisqu'ils représentent entre 10 et $15 \%$ de l'outillage (Denis 2014a, p. 212). Néanmoins, une partie est toujours réalisée sur lame. On peine à trouver une explication sauf à envisager une faible représentation du travail des peaux et donc des sphères d'activités différentes de celles connues sur les autres sites d'habitat du Bassin parisien. Parmi l'outillage sur lame, la place prépondérante du burin est, elle, plus traditionnelle, cet outil pouvant même représenter plus de la moitié des outils sur lame (ibid.). Le travail des plantes est toujours bien représenté mais ici un nombre important de burins réalisés sur éclats et qui viennent s'ajouter aux outils sur lame - ce qui est inhabituel - renforce la place de cette sphère d'activités. La présence de nombreux éléments de faucille, dont certains sont ici aussi sur éclat, atteste d'activités agricoles intensives. On peut s'interroger sur la signification de ce transfert de certaines activités traditionnellement réalisées avec des supports laminaires vers les éclats, sans doute à la jonction entre un déficit de support disponible et un domaine d'activité en pleine expansion. Au sein des outils sur éclat, la présence de tranchets a été observée sur trois des sites (Loisonsous-Lens, Vitry-en-Artois et Boves) et son absence soulignée 
à Languevoisin-Quiquery. Cet outil apparaît tardivement dans le Bassin parisien, à la phase finale du BVSG, alors que sur les sites du Hainaut, cet outil est attesté sur trois sites dont Irchonwelz « la Bonne Fortune » attribué à la phase ancienne du BVSG (Constantin et al. 1978, Denis 2014a), mais aussi à Aubechies et à Ellignies-Sainte-Anne. On pourrait alors, si l'on admet la synchronie des phasages entre le Hainaut belge et le Bassin parisien, considérer que le tranchet fait son apparition dès la phase ancienne dans le Hainaut belge et est assimilé plus tardivement à la panoplie d'outils dans le Bassin parisien. L'absence de ce type d'outil à Languevoisin-Quiquery reste néanmoins un fait plus difficilement compréhensible puisque cette occupation est datée de la fin du BVSG, à moins de considérer une nouvelle fois un statut original de cette occupation lié à la fonction du site. Dans ce cadre, la présence de produits laminaires de second choix (corticaux et sous crête) prendrait toute sa signification.

Revenons, pour terminer, sur les armatures de flèches qui, rappelons-le, proviennent presque exclusivement du site de Loison-sous-Lens (une seule autre à Vermand). Le type traditionnel dans le Bassin parisien, perçante à retouches inverses sur la base, est bien présent et associé en quantité égale aux flèches tranchantes. Si dans le Bassin parisien, ces dernières apparaissent aussi massivement à la phase finale du BVSG, sur les sites du Hainaut, quelques exemplaires sont mentionnés sur le site d'Irchonwelz « la Bonne Fortune » (Farruggia et al. 1982) et à Blicquy « la Couture du Couvent » (Constantin et al. 1991), donc dès la phase ancienne. Rappelons aussi que les trapèzes sont les types d'armatures les plus représentés dans les phases anciennes du Rubané oriental (Gronenborn 1990, Stadler et Kotova 2013).

\section{LE MACRO-OUTILLAGE LITHIQUE : LES MEULES DE LOISON-SOUS-LENS}

Dans notre région d'étude, la découverte d'un nombre particulièrement important d'outils de mouture, associés à des déchets de fabrication à Loison-sous-Lens (93 objets en grès pour un poids de $165 \mathrm{~kg}$ ), constitue la principale source d'information. Elle contraste avec l'indigence du mobilier en grès, retrouvé dans la vallée de la Somme, à Sancourt (un éclat de ravivage d'outil de mouture) ou plus en aval à Boves (trois éléments en grès; fig. 38).

À l'échelle régionale comme à celle du BVSG, l'industrie en grès de Loison-sous-Lens se révèle une série de références tout à fait exceptionnelle à plus d'un titre. Hormis un outil indéterminé (ST 1119) et un probable abraseur à rainure (st. 1009), tous les vestiges en grès sont liés à l'activité de broyage. Au total, le site a livré douze meules, dix molettes, cinq fragments d'outils de broyage ou vestiges de leur fabrication (4 éclats d'entame, 41 éclats de façonnage) et de leur entretien (6 éclats de ravivage). Ces outils proviennent de six structures différentes (tabl. 7). La présence d'appariements de meules et molettes, et la conservation entière de la quasi-totalité des 22 meules et molettes, sont tout à fait exceptionnelles pour le BVSG. Ceci nous a conduits à présenter en détail le contenu et les caractéristiques des outils de chaque assemblage séparément, afin d'aborder de façon plus synthétique quatre questions principales : la diversité typologique des meules, la fonction de ces outillages, le caractère éventuellement spécialisé du site dans la préparation alimentaire par comparaison avec des sites d'habitats « classiques » et enfin la signification des pratiques de dépôt ou d'abandon d'outils de mouture en grandes quantités sur les sites d'habitats BVSG.

En effet, l'extrême Nord de la France constitue une région clef pour la compréhension de l'articulation des deux groupes régionaux composant la culture de BVSG telle que définie par C. Constantin (Constantin et al. 1978 et 1991) et Y. Lanchon (Lanchon 2008). Par ailleurs, cette région se situe au centre de l'aire de répartition des " dépôts » de meules, plus particulièrement documentés dans le Hainaut (Constantin et al. 1978, Hamon 2008) et le bassin de la Seine (Hamon et Samzun 2004, Hamon 2008, Prestreau 1992), ce qui posait d'emblée la question de l'existence de telles manifestations sur la frange nord-occidentale.

\section{LES MATIÈRES PREMIÈRES EXPLOITÉES POUR LA CONFECTION DES OUTILS DE MOUTURE}

Les grès employés pour la confection des meules et molettes de Loison-sous-Lens présentent une silicification bien développée. Au moins deux types de grès différents ont été identifiés macroscopiquement : des grès quartzitiques de teinte jaune, avec ou sans empreintes végétales fossiles largement majoritaires et des grès quartzitiques de teinte violacée (ST 11, meule $\mathrm{n}^{\circ} 1$; ST 1119, meule massive ; ST 1174, meule). La grande majorité des meules de Loison-sous-Lens sont confectionnées sur des plaques de grès quartzitiques très bien triés, qui présentent un litage horizontal marqué.

D'après les cartes géologiques de Béthune et Carvin au 1:50 000, les niveaux tertiaires de grès thanétiens (niveaux dits « landéniens », e2b-Sables et grès d'Ostricourt) affleurent ponctuellement autour de Loison-sous-Lens, notamment au sud et à l'est de Liévin et au nord d'Épinoy. Les sables et grès d'Ostricourt, en particulier le faciès du Quesnoy, semblent correspondre au faciès le plus exploité pour la réalisation des outils de Loison-sous-Lens : il s'agit de sables blancs, qui « peuvent contenir des grès blancs mamelonnés disposés en bancs discontinus ». Ces mêmes niveaux de grès ont été largement exploités pour la réalisation d'outils de mouture, selon des modalités et pour la réalisation de types différents, aux périodes postérieures notamment au Néolithique final (Monchablon 2015, p. 234) et à l'époque romaine (Reniere et al. 2016, p. 13).

Ces qualités de grès sont équivalentes à celles généralement employées dans les régions limitrophes du Hainaut (grès landéniens) et du nord du bassin de la Seine. On observe donc une homogénéité réelle au sein du BVSG dans le choix de grès aux propriétés mécaniques particulières, assurant à la fois une longévité d'utilisation en raison de leur dureté, une efficacité certaine dans l'activité de mouture liée à leur composante siliceuse et une faible contamination du produit fini du fait de leur forte cohésion. 
Fig. 38 - Outil de percussion sur galet. Boves «les Longues Haies" (TR 2 ST 2.7 ; C. Hamon, CNRS).

Percussion tool on a pebble. Boves "Ies Longues Haies" (TR 2 ST 2.7; C. Hamon, CNRS).
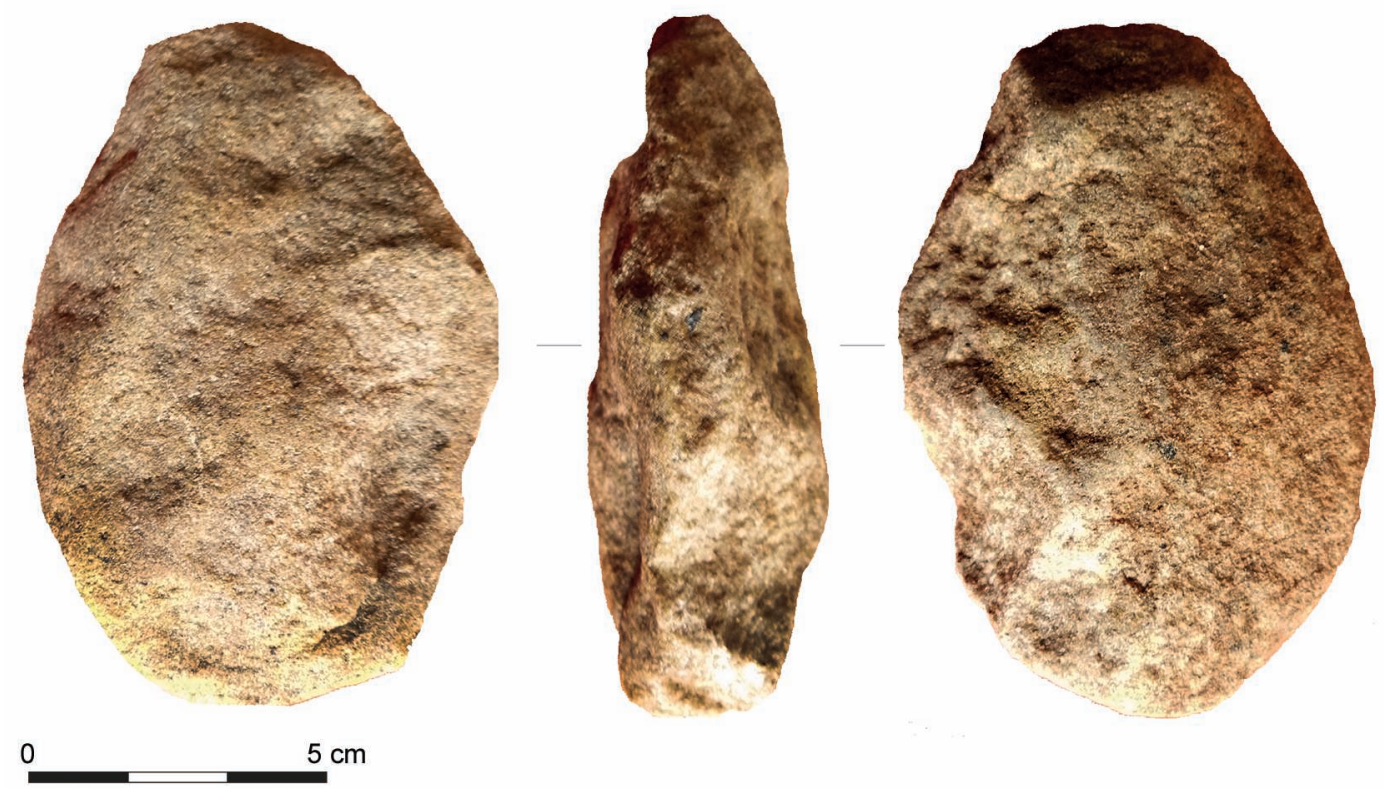

Tabl. 7 - Composition de l'outillage des fosses BVSG de Loison-sous-Lens (C. Hamon, CNRS).

Composition of the toolkit from the BVSG pits from Loison-sous-Lens (C. Hamon, CNRS).

\begin{tabular}{|c|c|c|c|c|c|c|c|c|c|c|c|c|c|}
\hline St & Meule & $\begin{array}{c}\text { Fragment } \\
\text { meule }\end{array}$ & Molette & $\begin{array}{l}\text { Ébauche } \\
\text { de molette }\end{array}$ & $\begin{array}{c}\text { Fragment } \\
\text { de molette }\end{array}$ & $\begin{array}{c}\text { Fragment } \\
\text { mouture }\end{array}$ & $\begin{array}{c}\text { Éclat } \\
\text { ravivage }\end{array}$ & $\begin{array}{l}\text { Abraseur } \\
\text { rainure }\end{array}$ & $\begin{array}{c}\text { Outil } \\
\text { indéterminé }\end{array}$ & Éclat & $\begin{array}{c}\text { Éclat } \\
\text { entamé }\end{array}$ & Fragment & Total \\
\hline 11 & 4 & & & 2 & & & 1 & & & 2 & & & 9 \\
\hline 1007 & & & & & & & & & & 3 & & 2 & 5 \\
\hline 1008 & & 2 & 2 & & 1 & 1 & 3 & & & 20 & 3 & 7 & 39 \\
\hline 1009 & & & & & & & & 1 & & & & & 1 \\
\hline 1031 & 1 & & & & & & & & & 2 & & 1 & 4 \\
\hline 1032 & & & & & 1 & & & & & 1 & & 1 & 3 \\
\hline 1049 & & & & & & & & & & 1 & & & 1 \\
\hline 1069 & & & & & & & & & & 2 & & 4 & 6 \\
\hline 1074 & 2 & & 2 & & & & & & & & & 1 & 5 \\
\hline 1078 & 1 & & 1 & & & & & & & 2 & & & 4 \\
\hline 1119 & 2 & & 1 & & & & 2 & & 2 & 5 & & & 13 \\
\hline 1137 & & & & & & & & & & 3 & & & 3 \\
\hline Total & 10 & 2 & 6 & 2 & 2 & 1 & 6 & 1 & 2 & 41 & 4 & 16 & 93 \\
\hline
\end{tabular}

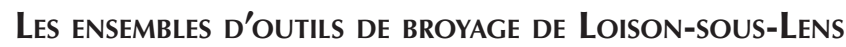

La conservation exceptionnelle et les quantités d'outils de broyage de Loison-sous-Lens interrogent d'emblée sur l'existence de possibles dépôts de meules dans plusieurs des structures du site. Afin de discuter cette hypothèse, les assemblages d'outils de mouture sont ici présentés structures par structures, afin de mettre en évidence des récurrences ou organisations particulières.

\section{La structure 11}

La structure 11 découverte lors du diagnostic en 2003 a livré l'ensemble d'outils de broyage le plus important du site : quatre meules entières, deux ébauches de molettes et trois éclats d'outils de mouture (Gaillard et al. 2003). Trois des meules entières ont été découvertes au décapage dans la partie supérieure de la fosse, la quatrième meule dans le fond de la fosse. Aucune organisation spécifique n'a pu être relevée à la fouille.

Une meule (fig. 39, A) a été abîmée au décapage. De forme ovoïde et de section semi-circulaire, sa surface active se distingue par une concavité prononcée et très régulière (de plus de $2 \mathrm{~cm}$ ), et un rebord proximal d'environ $4 \mathrm{~cm}$ de large grossièrement bouchardé et poli. Le modelage de sa surface externe par détachement d'éclats particulièrement soigné a préservé de larges plages brutes au dos de l'objet.

Une seconde meule (fig. 39, B) offre une morphologie particulière pour le BVSG, en raison de son étroitesse et de son épaisseur plutôt originale $(17 \mathrm{~cm}$ de large pour $18 \mathrm{~cm}$ d'épaisseur). Les deux bords droits sont bruts tandis que le dos et les extrémités sont façonnés par enlèvement. La surface active est délimitée par une retouche systématique. 

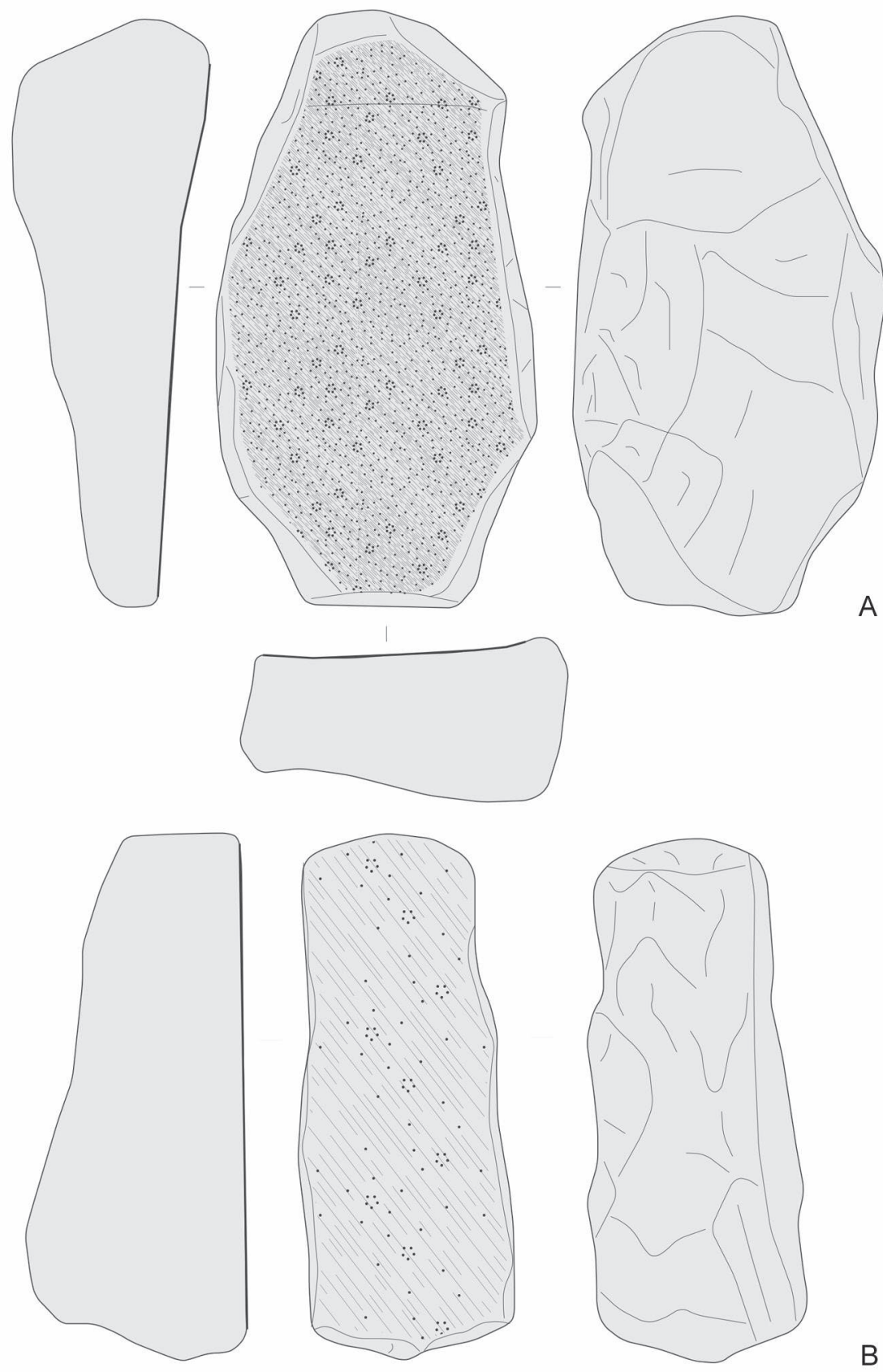

Une troisième meule (fig. 40, A) est fracturée obliquement sur l'un de ses bords et l'une de ses extrémités, mais devait présenter une forme et une section initialement quadrangulaires. Elle a été réalisée sur une plaque de grès compact à litage horizontal. Son dos est brut et ses bords ont été façonnés par détachements d'éclats. La surface active concave est très régulière et principalement lissée sur toute une bande périphérique. Le long d'un bord, l'usure déborde même largement au-delà de l'arête. L'analyse tracéologique montre qu'il s'agit d'une usure de type « pierre contre pierre », qui témoigne d'un frottement intensif de la molette sur cette partie de la meule. L'analyse tracéologique confirme par ailleurs son usage pour le broyage des céréales.

Enfin, une meule (fig. 40, B) a été façonnée sur une plaque de grès quartzitique, dont le plan de clivage constitue le dos de la pièce. Ses bords ont été grossièrement épannelés. La face supérieure, globalement plane, a été transformée partiellement en surface active : la périphérie de la face d'utilisation est en effet brute de toute transformation sur deux à trois centimètres de large. Il ne s'agit pourtant pas d'une ébauche de meule puisque une plage centrale présente un fin piquetage et un poli des aspérités. Son aspect relativement frais et peu profond démontre que la durée de vie de l'objet a dû être relativement courte. La disposition des plages piquetées et la répartition du poli suggèrent un geste au moins partiellement circulaire de broyage. L'analyse tracéologique de la surface active démontre son usage pour le broyage des céréales : si la relative préservation des grains constitutifs de la roche s'explique par un faible degré d'utilisation de la surface active, on observe un fort arasement général de la surface et le développement d'une pellicule superficielle à fort grossissement consécutif d'une action de mouture des céréales.

Les trois éclats de débitage de meules et les deux ébauches de molettes (fig. 40, C et D) qui accompagnent ces meules témoignent du déroulement d'étapes de façonnage des outils et de ravivage des surfaces actives des outils de broyage dans ce secteur au nord de la zone d'implantation reconnue. Ces deux ébauches de molettes ont été confectionnées à partir d'éclats d'un ou de plusieurs blocs de grès quartzitique. Malgré leur poids similaire (850 g environ), ces molettes correspondent à deux types et dimensions de molettes différents, actionnées respectivement à une ou deux mains. Leurs faces sont brutes et correspondent à d'anciens plans de clivage du bloc de départ. Le dos de ces ébauches est aménagé par des enlèvements couvrants, dont les arêtes ont été écrasées par martelage et polies. 
La structure 11 rassemble donc des meules de matières premières, types et degrés d'utilisation très différents, ainsi que des indices techniques disparates évoquant un façonnage et un entretien des outils à proximité : les seules molettes représentées le sont à l'état d'ébauche et au moins une des meules (fig. 40, B) présente une préparation tout à fait partielle. Cet assemblage, techniquement et typologiquement hétéroclite, semble cependant totalement dédié à l'activité de mouture des céréales. Si la concentration d'outils dans cette structure posait la question d'un éventuel dépôt de meules, plusieurs indices infirment cette interprétation. Aucune disposition particulière des outils de broyage n'a pu être dégagée, les objets ne sont pas déposés en connexion. En outre, aucune molette n'est appariée aux meules déposées. La présence de déchets de débitage et d'ébauches dans la structure 11 contraste avec ce que l'on retrouve généralement dans la plupart des dépôts de meules. Cette structure pourrait donc être interprétée plutôt comme une zone d'activité, de rejet, voire de stockage en lien avec la fabrication et / ou l'utilisation des meules.

\section{La structure 1008}

Le mobilier en grès de la structure 1008 (39 objets) comprend principalement deux molettes entières, plusieurs fragments d'outils (deux de meules et deux de molettes), ainsi que trois éclats d'entame de blocs et trois éclats de ravivage.

On compte deux grands fragments de meule (de plus de $3 \mathrm{~kg}$ ) : il s'agit dans un cas de l'extrémité semicirculaire d'une meule de petites dimensions (fig. 41, A) et dans le second cas d'un fragment résiduel de meule éclatée à la chauffe (négatifs d'enlèvements thermiques). L'un de ces fragments présente une usure indéterminée, nettement différente d'un broyage des céréales, et jusque-là non identifiée sur des outils archéologiques. L'arasement est uniforme, l'aspect de l'usure est " gras », les grains bien que fortement amalgamés sont bien individualisés. Ceci se rapprocherait des traces laissées par le broyage d'une substance humide de nature a priori plutôt minérale que végétale.

Les deux molettes entières présentent des morphologies et des modes de fabrication proches (fig. 41, B et C). La première molette a été fabriquée à partir d'une plaque de grès quadrangulaire, son dos est brut et ses bords grossièrement épannelés. Sa surface active présente des sillons transversaux et parallèles de piquetage. La seconde molette de forme ovoïde a été soigneusement façonnée sur toute sa surface externe. Sa surface active plane porte un lissage particulièrement prononcé, de même que son dos au poli de frottement marqué.

Les éclats présentent dans leur grande majorité des traces de chauffe et sont issus à la fois d'étapes d'entame de bloc et de façonnage ou ravivage d'outils. Ils constituent des déchets de fabrication tout à fait « classiques ».
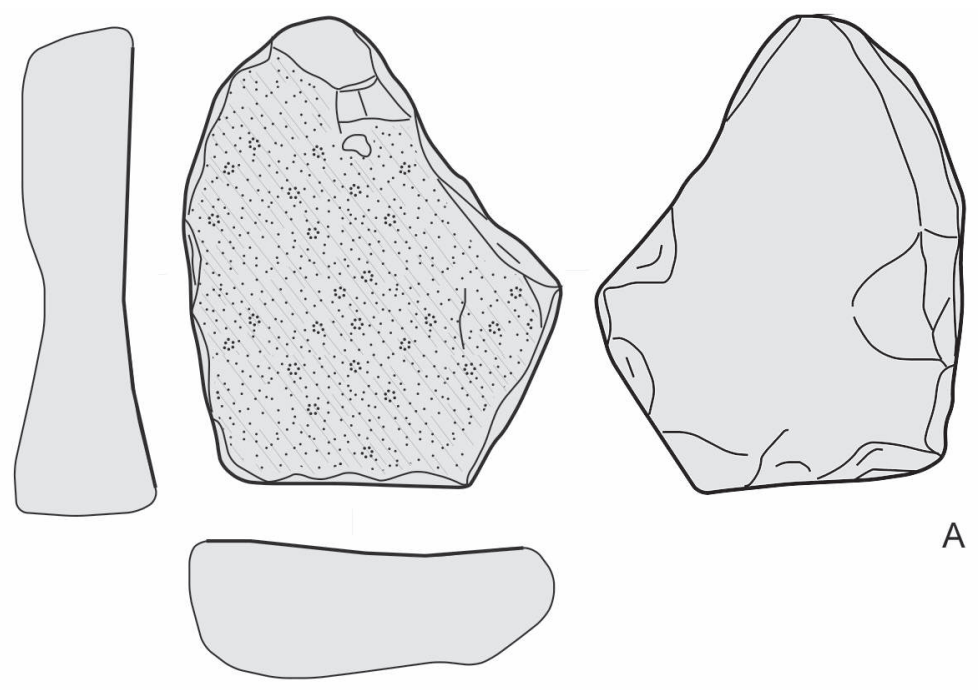

A
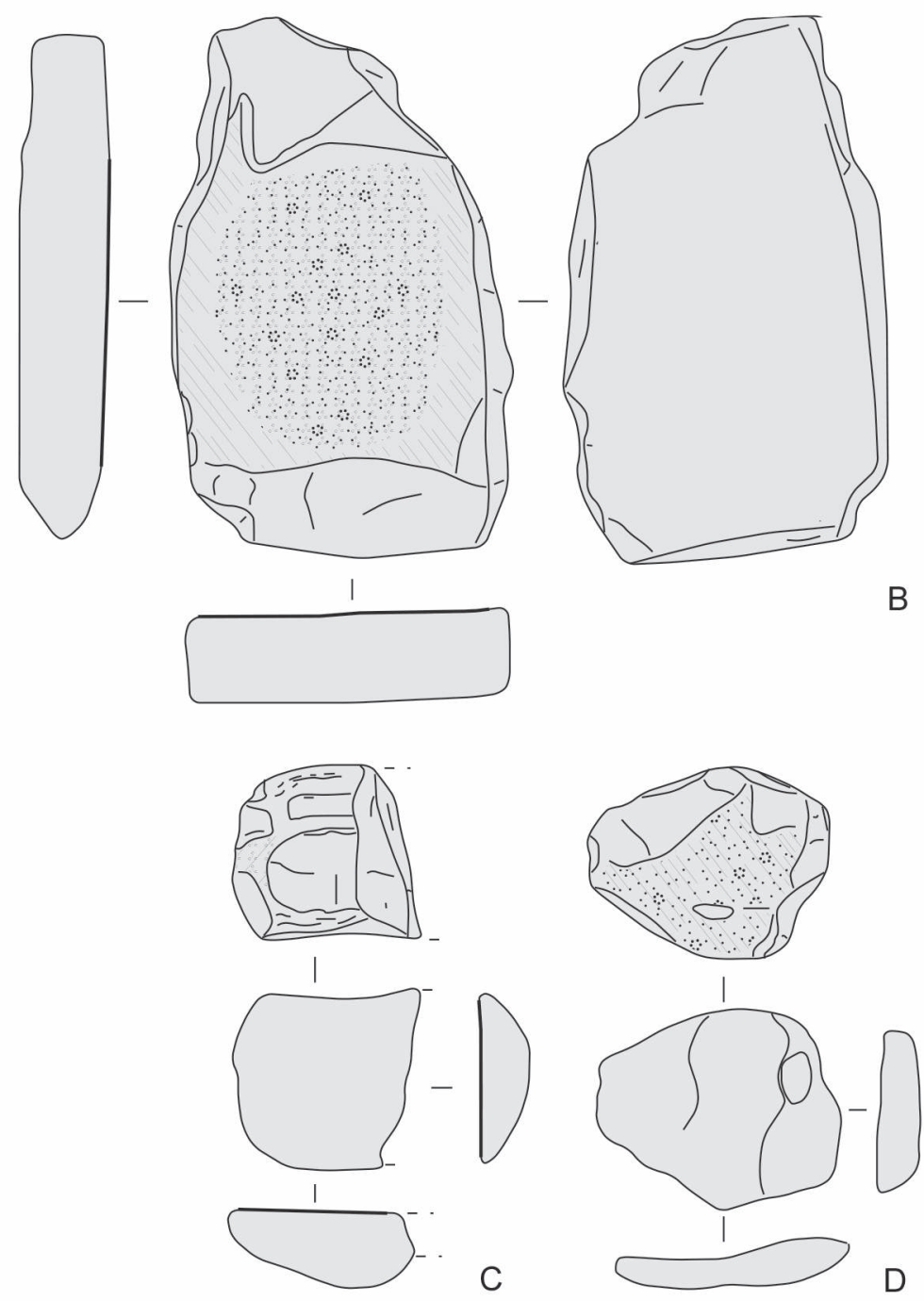

$0 \quad 10 \mathrm{~cm}$

Fig. 40 - Loison-sous-Lens. Structure 11 (C. Hamon, CNRS). Outils de mouture entiers : A-B. meules, C-D. ébauches de molettes.

Loison-sous-Lens. Structure 11 (C. Hamon, CNRS). Whole grinding tools: $A$-B. querns; $C-D$. grinder roughouts. 

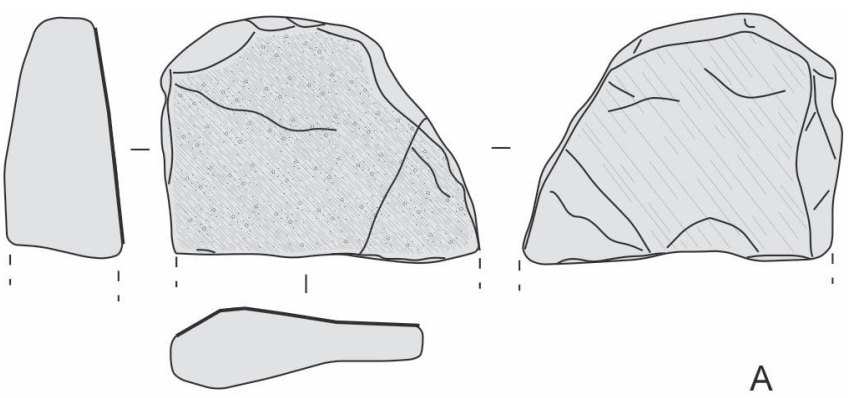

A
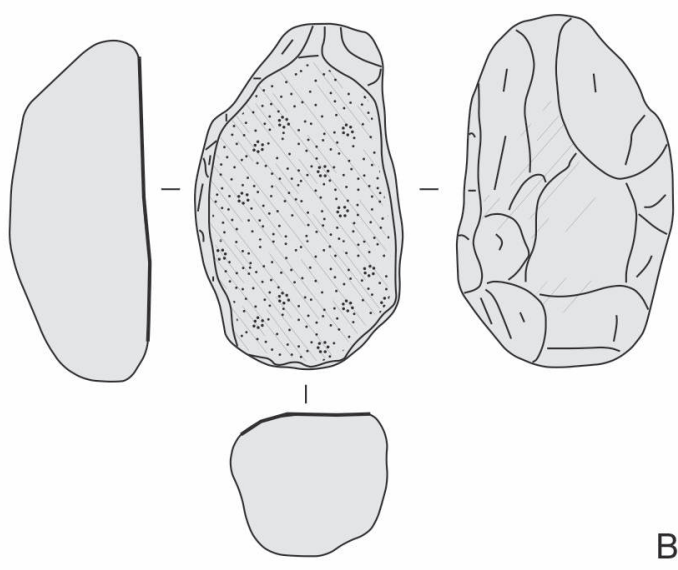

B
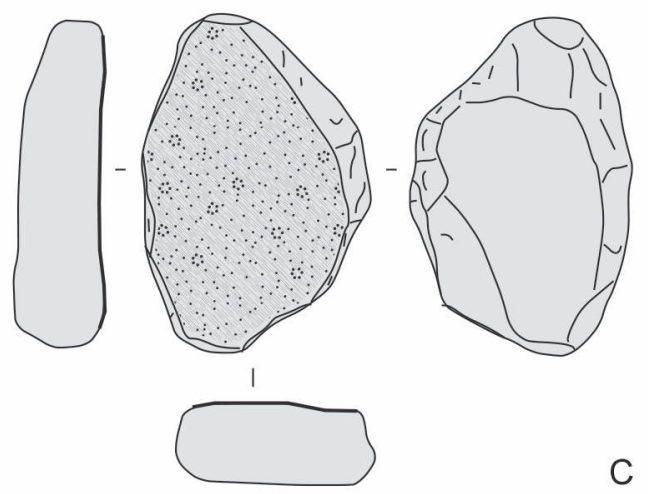

C

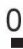
$10 \mathrm{~cm}$

Fig. 41 - Loison-sous-Lens. Structure 1008 (C. Hamon, CNRS) A. fragment de meule (NW p5), B. molette (NE p4), C. molette (US 2 p5). Loison-sous-Lens. Structure 1008 (C. Hamon, CNRS).

A. quern fragment (NW p5); B. grinder (NE p4); C. grinder (US 2 p5).

La composition du mobilier en grès de la structure 1008 est ainsi un peu plus proche de celle généralement retrouvée dans les fosses latérales des habitations BVSG, même si là encore seule l'activité de broyage est représentée.

\section{La structure 1031}

Lors du décapage de la structure 1031, perturbée lors de la première guerre mondiale, une meule fracturée en quatre morceaux et fortement altérée à la chauffe a été découverte.

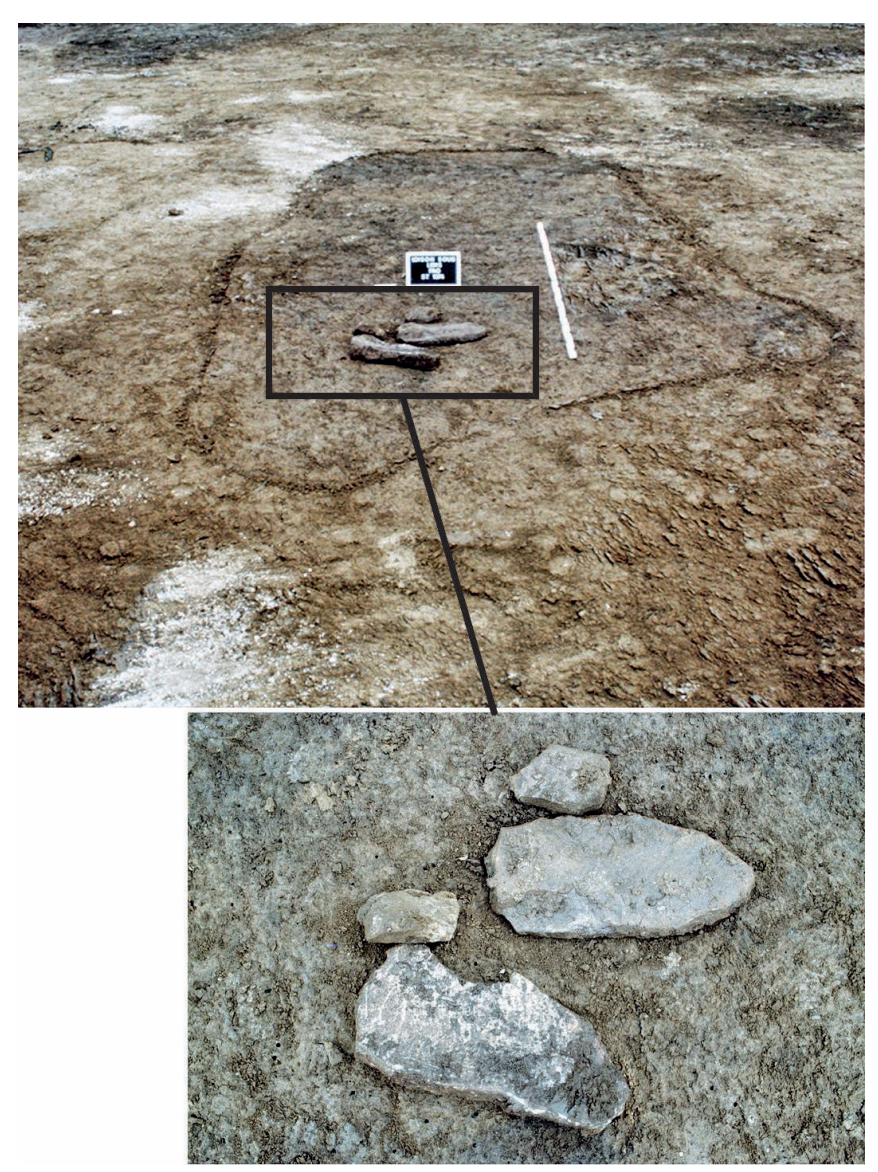

Fig. 42 - Position des meules et molettes lors du décapage de la structure 1074 (I. Praud, Inrap).

Position of querns and grinders during the discovery of structure 1074 (I. Praud, Inrap).

Losangique de section rectangulaire, elle présente une surface active plano-convexe grossièrement piquetée. Deux éclats et un fragment de grès y ont été retrouvés. Cette meule est donc seule déposée au sein de la fosse 1031.

\section{La structure 1074}

La structure 1074, peu profonde, a livré deux meules et deux molettes entières lors du décapage (fig. 42). La disposition des outils dans la fosse suggère qu'elles étaient vraisemblablement appariées.

La première meule est quasiment entière et n'a subi qu'une fracture partielle de l'un de ses bords (fig. 43, A). Élaborée à partir d'une plaque trapézoïdale de grès quartzitique, le centre de la surface active présente une concavité prononcée. Il semble que l'outil ait subi quelques aménagements visant à son remodelage partiel ou complet : l'extrémité de l'outil a été abattue afin de réduire sa concavité. Il porte également des fissurations typiques d'une chauffe. La surface active, inégalement usée, présente une bande périphérique de lissage, débordant de l'arête. Cette 

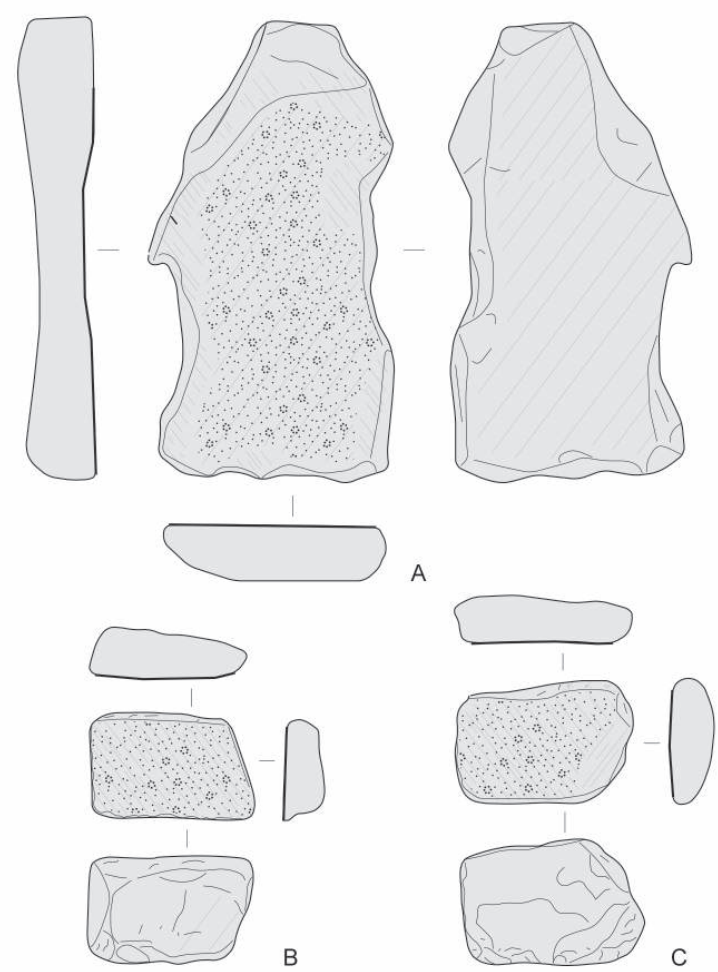

B

C
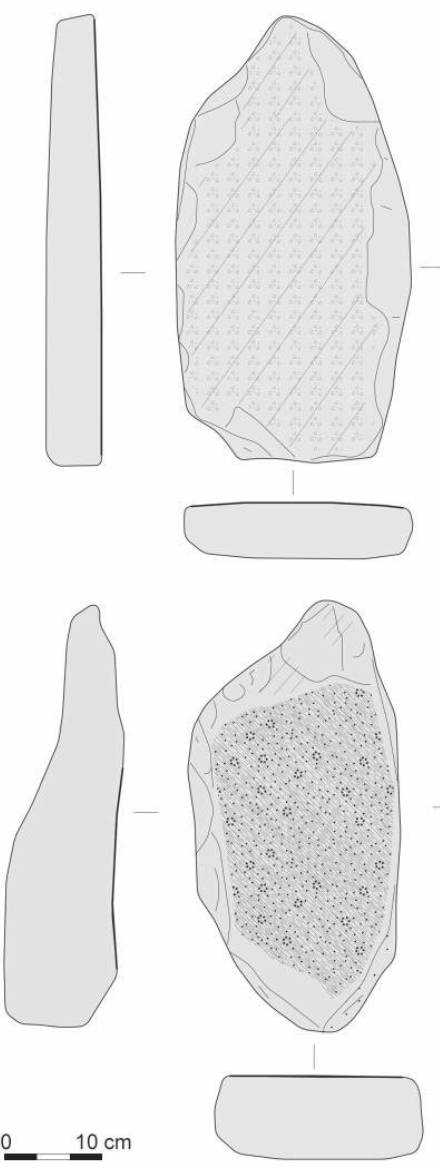

Fig. 43 - Structure 1074 (C. Hamon, CNRS). A. meule, B-C. molette.

Structure 1074 (C. Hamon, CNRS). A. quern; B-C. grinder.

dernière résulte d'un frottement de la molette plus accentué sur le bord de la meule qu'en son centre : les grains y sont fortement écrêtés et la surface arasée de façon uniforme.

La seconde meule a été réalisée sur une plaque litée en grès quartzitique violacé, de forme et de section quadrangulaire (fig. 44, A). Son dos a été préservé de tout aménagement et sa surface active n'a pas été préparée de façon régulière. Une bande périphérique irrégulière de quelques centimètres de large est restée brute de toute préparation : cette particularité pourrait donc relever de la structure litée de la matière première ou témoigner d'une phase de reprise des bords de la surface active. Cet objet se distingue assez significativement des autres meules du site mais semble bien avoir servi pour le broyage des céréales d'après l'analyse fonctionnelle.

Les deux molettes présentent des caractéristiques similaires : elles sont réalisées dans le même grès quartzitique, ont des dimensions similaires et des schémas de façonnage comparable, avec un aménagement des bords ou du dos par façonnage autour de plages préservées brutes (fig. 43, $\mathrm{B}$ et $\mathrm{C}$ ). L'une des deux molettes de forme quadrangulaire présente une fine retouche de délimitation de la surface active et un fin piquetage transversal associé à une usure peu développée. L'analyse tracéologique confirme son usage pour le broyage de céréales.

\section{$0 \quad 10 \mathrm{~cm}$}

Fig. 44 - Structure 1074 (C. Hamon, CNRS). A. meule, B. table de broyage.

Structure 1074 (C. Hamon, CNRS). A. quern; B. grinding table.

\section{La structure 1078}

La structure 1078 a livré une meule et une molette entières, ainsi que deux éclats. La meule retrouvée dans la partie supérieure du remplissage de la fosse a été endommagée par la pelle mécanique au niveau de son extrémité proximale. Son autre extrémité a subi un remodelage par détachement d'éclats périphériques lui conférant une forme appointée, sur laquelle impacts de bouchardage épars et plages de lissage sont bien visibles. Particularité de cette meule, sa surface active a été préparée par un grossier martelage, à l'origine d'une étendue plus importante des plages polies. D'après l'analyse tracéologique, cette meule ne semble pourtant pas correspondre à une fonction autre que celle du broyage des céréales. L'unique molette quadrangulaire rappelle l'une des molettes de la structure 1074. Elle a été façonnée uniquement sur ses bords : par détachement d'éclats sur l'un des bords, et par piquetage et polissage sur un autre bord. Sa surface active est délimitée par un martelage de retouche et est lissée aux deux extrémités. Les deux objets ne semblent pas avoir fonctionné ensemble ou avoir été disposés selon une organisation particulière, ce qui exclurait l'idée d'un dépôt de meules. 


\section{La structure 1119}

La structure 1119 a été découverte lors de la phase d'évaluation de la parcelle (Praud et al. 2005, ancienne ST 50). Elle a livré au total deux meules, une molette, deux outils indéterminés et huit éclats (deux de ravivage et un d'entame).

La première meule, retrouvée dans la partie sud-ouest de la fosse, se distingue par ses grandes dimensions $(43 \mathrm{~cm}$ de long pour $23 \mathrm{~kg}$; fig. 45, A). Elle a été confectionnée sur une plaque de grès quartzitique litée horizontalement. Son extrémité a été fracturée. Son dos est brut d'extraction tandis que ses bords ont été soigneusement façonnés et retouchés. De forme et de section quadrangulaire, sa surface active est particulièrement concave. Les traces visibles sur la surface active témoignent d'une durée d'utilisation importante et de plusieurs étapes de la vie de l'objet. Le pourtour totalement lissé de la surface active pourrait d'ailleurs résulter d'une utilisation primaire de la meule. Après un ravivage visible, il semble que le centre de la surface active ait fait l'objet d'un ravivage et d'une utilisation secondaire en percussion posée circulaire.

Une autre meule a été confectionnée sur une plaque de grès quartzitique. De forme trapézoïdale et de section quadrangulaire, sa surface active est plano-concave (fig. 45, B). Ses bords ont été assez grossièrement épannelés mais son dos a été préservé de tout façonnage et ne porte que des traces de frottement.

Une molette quadrangulaire en grès quartzitique a été façonnée sur ses bords, ensuite finement régularisés par piquetage, puis de petits enlèvements ont permis de retoucher l'arête le long de la surface active (fig. 45, C). L'arête de la surface active est systématiquement et finement retouchée. L'analyse tracéologique des zones lissées montre que les grains sont totalement amalgamés et qu'une pellicule superficielle très développée recouvre l'ensemble de la surface. Elle aurait donc servi au broyage de grains, probablement déjà décortiqués. Cet objet semble avoir eu une longue durée d'utilisation et il est possible que les grains aient été humidifiés avant broyage.

Les cinq éclats correspondent à l'ensemble des étapes du façonnage des meules (entame de bloc, façonnage, ravivage d'outil de broyage).

La structure 1119 ne reflète là non plus aucune des caractéristiques de composition ou de disposition des outils généralement rencontrés dans les dépôts de meules.

À la lumière de ces observations, les ensembles d'outils de mouture découverts à Loison-sous-Lens ne peuvent être directement interprétés comme des dépôts de meules.

\section{Les OUTILS DE MOUTURE DE LOISON-SOUS-LeNS DANS LEUR CONTEXTE BVSG}

\section{Typologie}

Aucune logique de distribution particulière ou de regroupement par type n'a pu être observée et ne semble structurer les assemblages. Les meules retrouvées dans les fosses BVSG de Loison-sous-Lens sont de trois types différents : meules peu épaisses sur plaques trapézoïdales ou quadrangulaires ; meules épaisses à surfaces planes ou concaves avec ou sans rebord proximal ; meule étroite et épaisse. Toutes les molettes appartiennent à la catégorie dite courte et deux types ont pu être distingués : des molettes ovoïdes à surface active plane ou convexe ; des molettes quadrangulaires à bords sub-abrupts et surface active plane. Une même logique de mise en forme semble partagée par tous les outils : elle favorise la préservation des surfaces brutes sur leur dos et/ou flancs, et une mise en forme grossière par épannelage ou détachement d'éclats de régularisation des autres plages de l'outil.

Cette typologie des outils de Loison-sous-Lens s'inscrit pleinement dans celle des régions limitrophes. Elles trouvent des comparaisons souvent directes à la fois dans le Hainaut, à Irchonwelz ou Blicquy (Constantin et al. 1978, Hamon 2008), comme dans le nord du bassin de la Seine en particulier dans la plaine de France à Saint-Denis (Hamon et Samzun 2004), la vallée de l'Aisne à Trosly-Breuil, Bucy-leLong le Fond du Petit Marais ou la Fosse Tounise (Hamon 2006), la vallée de l'Oise à Longueil-Sainte-Marie (Bostyn et al. 2015) ou encore la vallée de la Marne à Ocquerre (Hamon 2009) et Luzancy (Hamon 2013). La fabrication de meules quadrangulaires ou trapézoïdales sur plaques peu épaisses est ainsi documentée à Blicquy et Irchonwelz tout comme à Longueil, Saint-Denis et Ocquerre. Ce type serait plus fréquent, voire caractéristique des productions de la moitié nord du bassin de la Seine et du Hainaut, puisqu'il semble à l'heure actuelle absent de la vallée de la Seine aval, de la basse vallée de la Marne et de celle de l'Yonne (Hamon 2003, 2006 et 2013). Ceci pourrait en partie être lié à la configuration des faciès locaux exploités dans chacune de ces régions, qui présentent un litage plus ou moins marqué et plus ou moins épais. Les meules plus massives, de forme sub-quadrangulaire ou ovalaire, sont quant à elles beaucoup plus fréquentes dans toute l'aire BVSG. Au contraire, les meules étroites n'ont été identifiées que très ponctuellement sur quelques occupations, notamment à Blicquy. Elles ne peuvent cependant être considérées comme un marqueur régional puisqu'on en retrouve également beaucoup plus loin comme à Passy Sablonnière et Villeneuve-la-Guyard (Hamon 2006). Les deux types de molettes sont caractéristiques de ce qu'on retrouve dans le BVSG. Enfin, il faut noter la présence, plutôt rare mais répétée, de meules réutilisées en broyage circulaire sur trois sites du nord du bassin de la Seine : à Trosly-Breuil, Longueil et Saint-Denis. En ce qui concerne ces deux derniers sites, l'un est un dépôt de meule avéré, l'autre un dépôt de meules probable.

\section{Dimensions}

À l'examen des dimensions des outils, deux groupes de meules se dégagent nettement : un groupe de meules autour de 9 à $10 \mathrm{~kg}$ et un second groupe de meules dont le poids oscille entre 18 et $22 \mathrm{~kg}$ (fig. 46). L'épaisseur des meules les plus massives est comprise entre 12 et $18 \mathrm{~cm}$. De la même 

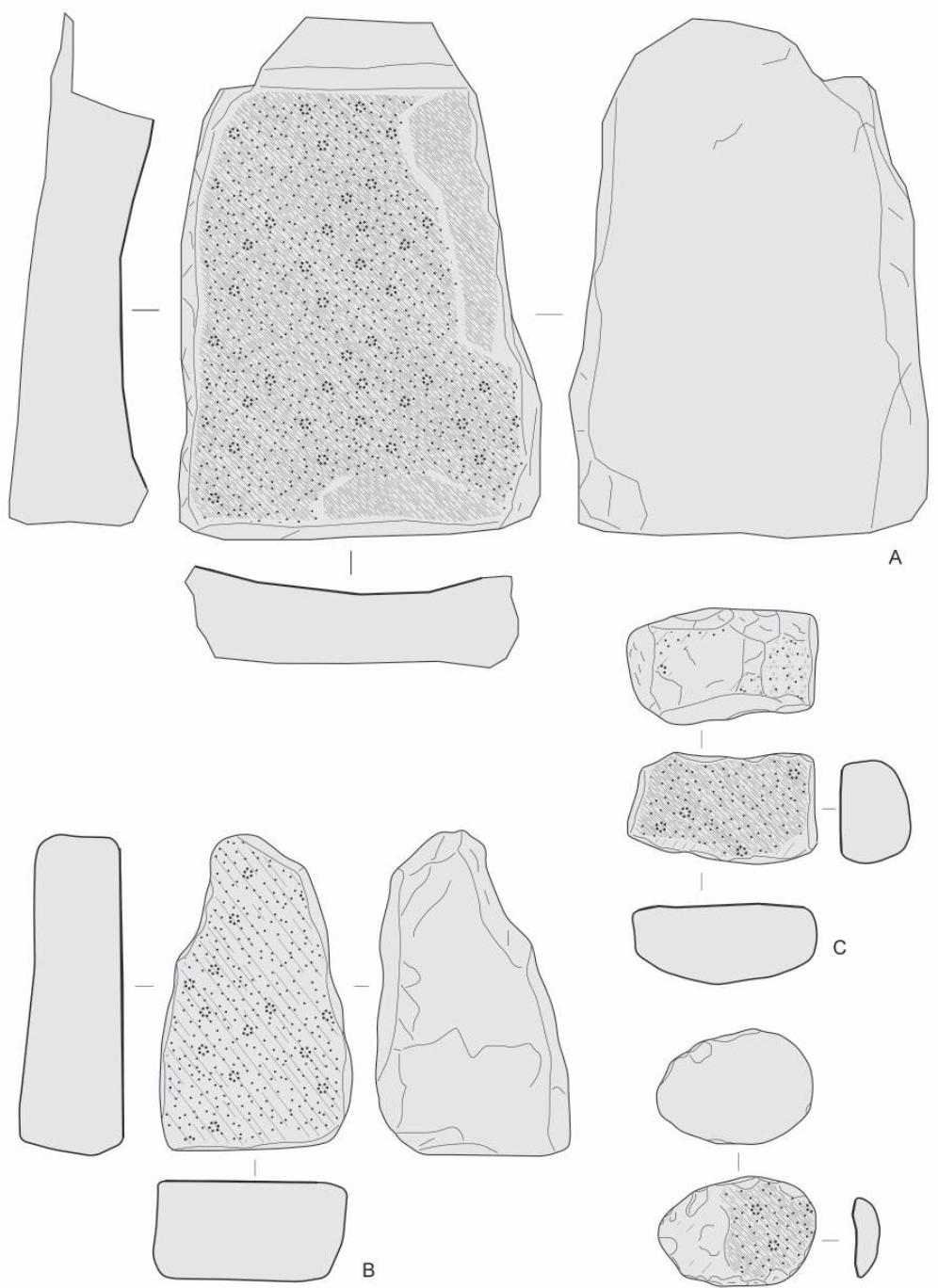

B
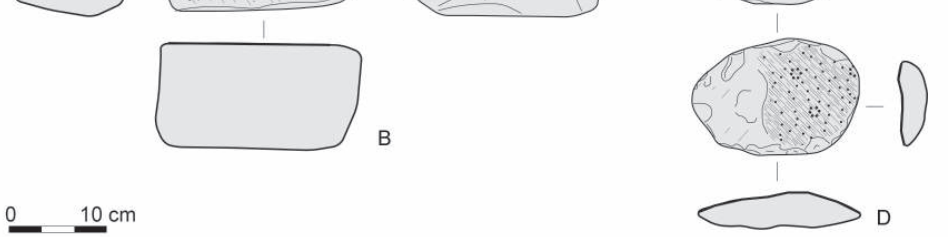

Fig. 45 - Structure 1119 (C. Hamon, CNRS). A. meule (SW), B. meule (NW p1), C. molette, D. outil indéterminé (NE).

Structure 1119 (C. Hamon, CNRS). A. quern (SW); B. quern (NW p1); C. grinder; D. undetermined tool (NE).

manière, on observe deux groupes bien distincts de dimensions de molettes. Un premier groupe inclut des molettes de 15 à $19 \mathrm{~cm}$ de long pour un poids inférieur à $1800 \mathrm{~g}$. Le second groupe de molettes mesure entre 22 et $24 \mathrm{~cm}$ de long, pour un poids compris entre $2,8 \mathrm{~kg}$ et $3,9 \mathrm{~kg}$. Les meules de Loison-sous-Lens sont donc globalement de plus grandes dimensions que les meules retrouvées en contexte détritique VSG. Leur longueur est comprise dans une moyenne entre celle des dépôts blicquyens d'Irchonwelz et BVSG de SaintDenis. Les meules de Loison-sous-Lens se distinguent par contre par une certaine étroitesse, leur largeur ne dépassant que rarement les $25 \mathrm{~cm}$. En cela, les meules de Loison-sousLens se rapprochent plus des outils classiquement retrouvés en contexte détritique BVSG que des meules des dépôts d'Irchonwelz ou de Saint-Denis. Les dimensions des deux
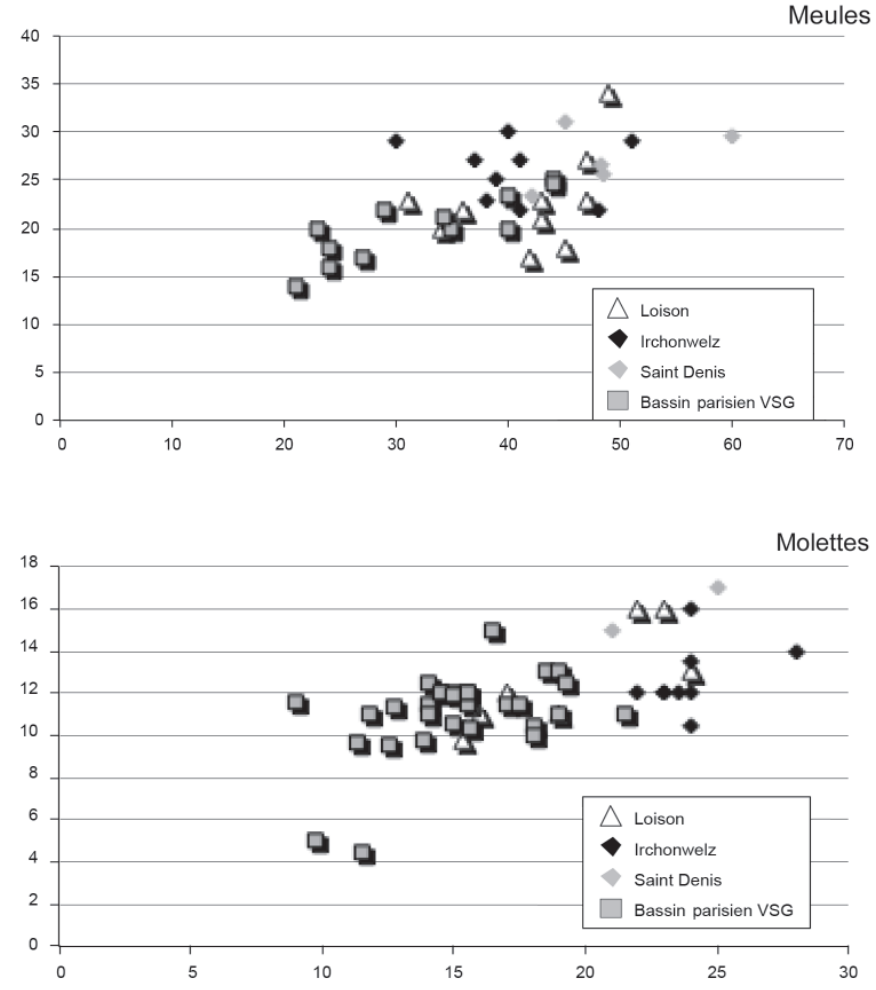

Fig. 46 - Dimensions des meules et molettes de Loison-sousLens comparées à celles des outils BVSG d'Irchonwelz, SaintDenis et du bassin de la Seine (C. Hamon, CNRS).

Dimensions of querns and grinders from Loison-sous-Lens compared to the BVSG querns and grinders from Irchonwelz, Saint-Denis and the Seine Basin (C. Hamon, CNRS).

groupes de molettes de Loison-sous-Lens correspondent respectivement à celles des contextes détritiques BVSG et à celles du dépôt de Saint-Denis. Cette division des systèmes de broyage en deux classes de dimensions n'est pas propre au BVSG et se retrouve autant dans les assemblages rubanés, que sur les sites du Néolithique moyen et final du Bassin parisien (Monchablon 2005 et 2015).

On dénombre quatre probables appariements de meules et de molettes (ST 1074, 1078 et 1119 ; fig. 47 et tabl. 8). Le rapport largeur meule / longueur molette est compris entre 1,2 et 1,8 avec une moyenne autour de 1,5. Ce rapport, classique pour les outils BVSG du Bassin parisien, confirme que dans tous les cas il s'agit de molettes de type " court ", dont la longueur est inférieure à la largeur de la meule. Il convient de s'interroger cependant sur l'étroitesse de l'une des meules de la fosse 11, qui a inévitablement une incidence sur son fonctionnement : dans la mesure où aucune distribution particulière de l'usure n'est visible (lissage débordant sur l'arête notamment), cette meule devait bien fonctionner avec une molette courte, de petites dimensions, manipulée à une main.

Le rapport longueur molette / longueur meule pour les couples d'outils appariés est de 0,3 à 0,4 , tandis que le rapport largeur molette / largeur meule est lui plus fluctuant, 
Tabl. 8 - Dimensions et matières premières des outils de mouture par fosses (C. Hamon, CNRS).

Dimensions and raw materials of grinding tools by pit (C. Hamon, CNRS).

\begin{tabular}{|c|c|c|c|c|c|c|c|}
\hline & L & I & e & $p$ & & I/L & L/I \\
\hline \multicolumn{5}{|l|}{ ST 1074} & & & \\
\hline Meule & 47 & 23 & 6 & 9000 & & & \\
\hline Mol & 16 & 11 & 7 & 1805 & & & \\
\hline Meule & 43 & 23 & 10 & 10100 & $\mathrm{Me} 1 / \mathrm{mol} 1$ & 0,34 & 0,48 \\
\hline Mol & 15,3 & 9,8 & 5,8 & 1605 & $\mathrm{Me} 2 / \mathrm{mol} 2$ & 0,36 & 0,43 \\
\hline \multirow{2}{*}{\multicolumn{8}{|c|}{$\begin{array}{l}\text { ST } 1078 \\
\text { Meule }\end{array}$}} \\
\hline & & & & & & & \\
\hline Mol & 17 & 12 & 11 & 1520 & $\mathrm{Me} 1 / \mathrm{mol} 1$ & 0,4 & 0,5714 \\
\hline \multirow{2}{*}{\multicolumn{8}{|c|}{$\begin{array}{l}\text { ST } 1119 \\
\text { Meule }\end{array}$}} \\
\hline & & & & & & & \\
\hline Meule & 34 & 20 & 11 & 9700 & $\mathrm{Me} 1 / \mathrm{mol} 1$ & 0,39 & 0,3235 \\
\hline Mol & 19 & 11 & 9 & 2545 & $\mathrm{Me} 2 / \mathrm{mol} 2$ & 0,56 & 0,55 \\
\hline
\end{tabular}

compris entre 0,3 et 0,5 . Dans le cas de la fosse 1119 , le calcul de cet indice permet d'attribuer plus vraisemblablement la molette à une meule plutôt qu'à une autre (indice de 0,39 pour la meule de plus grand gabarit, contre 0,56 pour la meule de plus petit gabarit).

L'imbrication des surfaces actives des meules et molettes est relativement correcte, sans être prononcée. Les molettes sont en effet relativement échangeables d'une meule à une autre. Ceci est dû aux dimensions relativement homogènes des outils entre les fosses et au faible degré de concavité des outils dormants, les surfaces actives des meules présentant plutôt un profil plan.

\section{Cycles d'utilisation, fonctionnement et fonction}

L'usage de systèmes de broyage à molettes courtes en va-et-vient est donc la règle à Loison-sous-Lens, même si un usage secondaire en broyage circulaire a été mis en évidence pour au moins une meule, et probablement deux autres. L'analyse tracéologique réalisée sur cinq meules et trois molettes montre un usage dédié à la transformation des céréales, en particulier à leur mouture. Un seul fragment de meule peu diagnostic typologiquement présente une usure indéterminée, plutôt en lien avec l'utilisation de matières minérales. L'outillage de broyage s'inscrit dans des cycles d'utilisation assez disparates, allant de l'utilisation de courte durée de meules à peine façonnée à l'utilisation intensive d'outils de mouture ayant subi des étapes de remodelage et de réutilisation. Le dos de plusieurs meules montre un lissage de frottement marqué, indiquant de longues durées d'utilisation. Aucune généralité sur le degré d'utilisation ou les cycles d'utilisation des meules à l'échelle de l'ensemble de l'occupation BVSG de Loison-sous-Lens n'est donc observée.

\section{LA PLACE de L'ACTIVITÉ DE MOUTURE SUR LE SITE DE LOISON-SOUS-LENS}

À la lumière du matériel en grès recueilli, plusieurs questions se posent concernant la composition et la nature de l'assemblage. Pourquoi ne trouve-t-on que des vestiges d'outils de broyage dans les fosses, à l'exclusion de quasiment tout autre outil en grès généralement présent dans les fosses latérales d'habitation BVSG (broyons, abraseurs, etc.) ? La rareté des éléments d'outils macrolithiques en grès semble faire écho à la pauvreté générale du mobilier de ce site.

Dans ce contexte, le nombre important de meules et leur conservation quasi systématiquement entière se révèlent donc doublement exceptionnels. Est-elle liée à l'état de conservation générale des fosses et des vestiges qu'elles contiennent ? Témoigne-t-elle d'un traitement particulier des meules de Loison-sous-Lens par rapport à ce qui est classiquement rencontré dans les fosses latérales d'habitation? Ou de la fonction ou du statut particulier des fosses BVSG du site ? Tout d'abord, la conservation des meules peut être, à première vue, qualifiée d'exceptionnelle. En dehors de quelques fosses ayant livré un remplissage détritique plutôt classique, on observe une relative diversité dans les configurations. La fosse 11 livre un nombre important d'indices techniques : présence de deux ébauches et d'éclats de réavivage associés à quatre meules dont une en cours de façonnage, une fracturée et une d'un type particulier (meule étroite et épaisse). Le contenu de la fosse 1074 est lui plus homogène avec ses deux couples de meules et molettes appariées. La fosse 1119 présente plusieurs éclats de ravivage et déchets de fabrication, ainsi qu'une grande meule concave fracturée et deux outils de nature indéterminée. À Loison-sous-Lens, les meules ont donc généralement été découvertes lors de la phase de décapage, dans la partie sommitale des fosses, et ne semblent pas en position détritique classique. De même, l'absence d'organisation spécifique de ces meules semble également exclure des dépôts de meules en place. Si nous n'avons pas affaire à une configuration classique de rejet ou à un dépôt, il faut alors envisager que les outils de broyage aient été abandonnés ou rejetés dans ces fosses lors d'une phase ultime de leur remplissage, et que certaines retrouvées dès le décapage, puissent même être en position d'utilisation. On peut dès lors envisager qu'il s'agissait soit d'anciens dépôts de meules démantelés progressivement au cours du temps, soit plutôt d'une zone de rejet d'outils de broyage abandonnés quasiment dans leur position d'utilisation associés à une partie des déchets de leur fabrication et entretien.

L'activité de mouture apparaît donc omniprésente sur le site de Loison-sous-Lens, du moins en regard de ce que l'on retrouve " classiquement » sur les sites d'habitat BVSG et on ne doit pas écarter une spécialisation même partielle des activités du site. On peut d'ores et déjà exclure qu'il s'agisse d'un site de fabrication de meules, dans la mesure où toutes les meules du site ont été utilisées et que les vestiges d'étapes de fabrication (éclats d'entame, grands éclats d'épannelage des bords et du dos) sont finalement rares dans les fosses. 

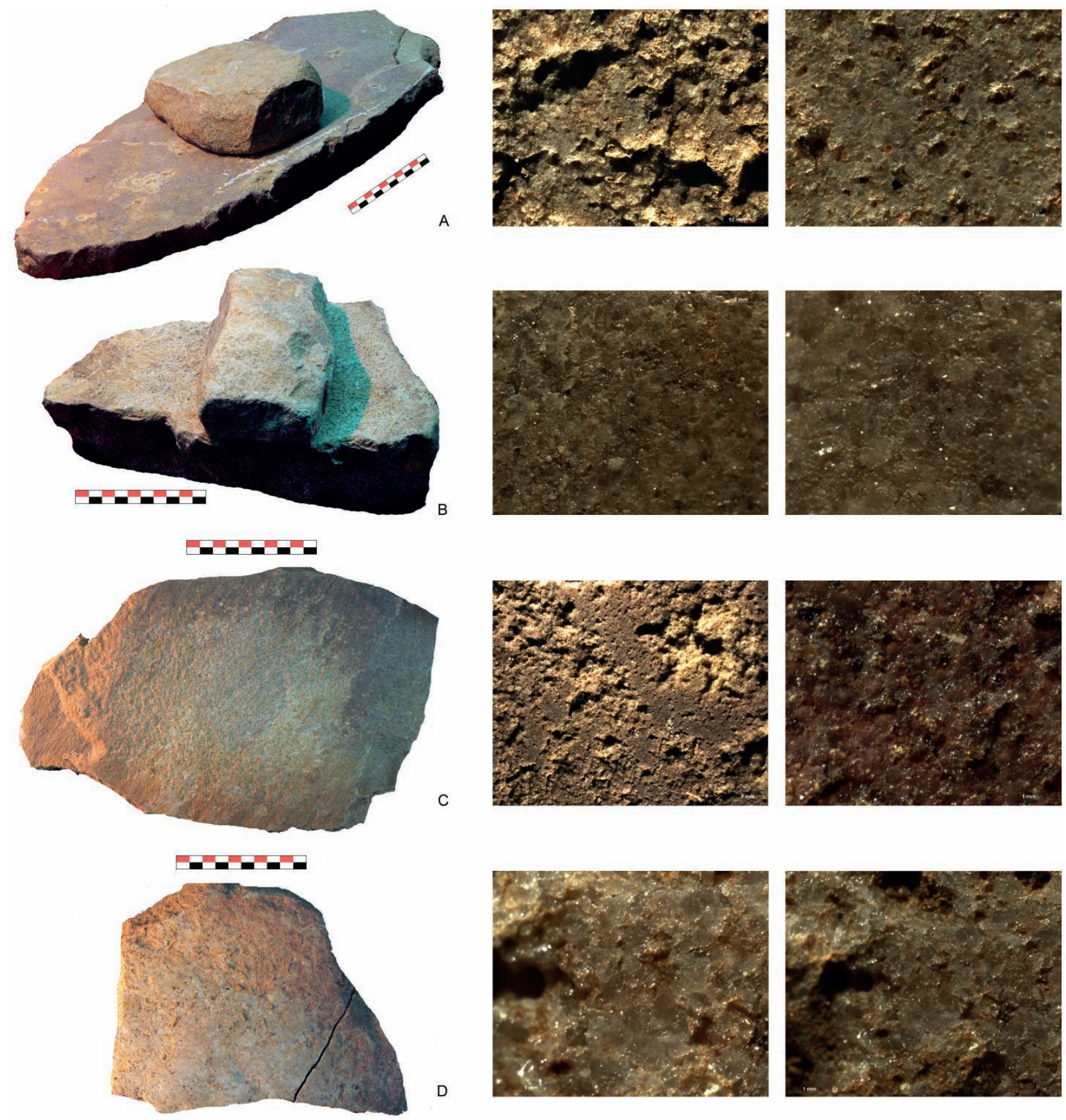

Fig. 47 - Propositions d'appariement de certaines meules et molettes, avec traces d'utilisation sur meules (C. Hamon, CNRS). A. meule ST 1074 : appariement, traces d'utilisation $x 5$ et $x 50$; B. meule ST 1119-ST 50 : traces d'utilisation $x 35$ et $x 60$; C. meule ST 11 : traces d'utilisation x 5 et x 30 ; D. meule ST 1008 : appariement, traces d'utilisation x 30 et x 60 .

Proposal of quern and grinder pairs, with use-wear traces on querns C. Hamon, CNRS).

A. quern ST 1074: pair, use-wear traces $x 5$ and x50; B. quern ST 1119-ST 50: use-wear x35 and x60; C. quern ST 11: use-wear traces $x 5$ and x30; D. quern ST 1008: pair, use-wear traces $x 30$ and $x 60$.

Les ébauches de molettes semblent être confectionnées sur de grands éclats de blocs et peuvent donc être considérées comme des réutilisations plus ou moins opportunistes. De même, les deux meules sur plaques ont pu être avivées juste avant leur utilisation et ont pu être confectionnées en dehors du site. Le façonnage grossier de certaines meules, la présence de formes atypiques (meule $\mathrm{n}^{\circ} 4$ de la fosse 11) et l'avivage frais et partiel de certaines surfaces actives (ST 11 et 1074) témoigneraient d'une forte demande en outils de broyage sur le site. Un épisode d'intense utilisation des meules pour la mouture des céréales à la fin de l'occupation BVSG du site pourrait expliquer la configuration particulière observée. 


\section{LA PARURE EN PIERRE ET EN TERRE CUITE}

\section{Présentation de la série}

La série repose sur trente-trois fragments au total pour un nombre minimum d'individu évalué à dix-neuf bracelets. Proportionnellement, la grande majorité de la parure découverte est confectionnée dans des matériaux schisteux, le reste étant fabriqué en argile. La répartition de ces objets est inégale entre les sites étudiés avec un minimum d'un individu pour le site de Boves et un maximum de sept atteint par les gisements de Loison-sousLens et d'Étouvie (tabl. 9). Il s'agit essentiellement de produits finis, mais il faut signaler la présence d'un déchet de perforation en schiste (fig. 48, $\mathrm{n}^{\circ}$ 9) témoignant d'une activité d'usinage de bracelet à Loison-sous-Lens.

\section{LES MATIÈRES PREMIÈreS}

Le schiste, principale ressource exploitée, a été observé à l'œil nu. En ce qui concerne l'argile cuite, les observations ont été conduites sur cassure fraîche à la loupe binoculaire (grossissement x 8). La pâte argileuse comporte une fraction sableuse non négligeable, sans que les dégraissants ajoutés ne soient discernables : seuls subsistent des vacuoles vides dans la pâte suggérant l'ajout d'un dégraissant de type végétal, animal, ou minéral ayant aujourd'hui disparu.

Les lieux de découverte de la parure en schiste sont situés en dehors de toute possibilité d'acquisition directe de la matière première, les affleurements naturels les plus proches étant localisés à plusieurs dizaines, voire centaines de kilomètres. Nous distinguons plusieurs faciès :

- un schiste gris assez terne, dont le plan de schistosité est peu marqué ;

- un schiste gris clair comportant des minéraux sombres dispersés dans la matière et présentant un plan de schistosité marqué ;

Tabl. 9 - Répartition des matières premières (I. Praud, Inrap). Raw material distribution (I. Praud, Inrap).

\begin{tabular}{|c|c|c|c|c|c|}
\hline Site & NR & NMI & Schiste & Schiste tacheté & Terre cuite \\
\hline LSL & 14 & 7 & 3 & 1 & 3 \\
LQ & 11 & 4 & 3 & 1 & \\
ECM & 7 & 7 & 5 & & 2 \\
Boves & 1 & 1 & 1 & & \\
\hline Total & 33 & 19 & 12 & 2 & 5 \\
\hline
\end{tabular}
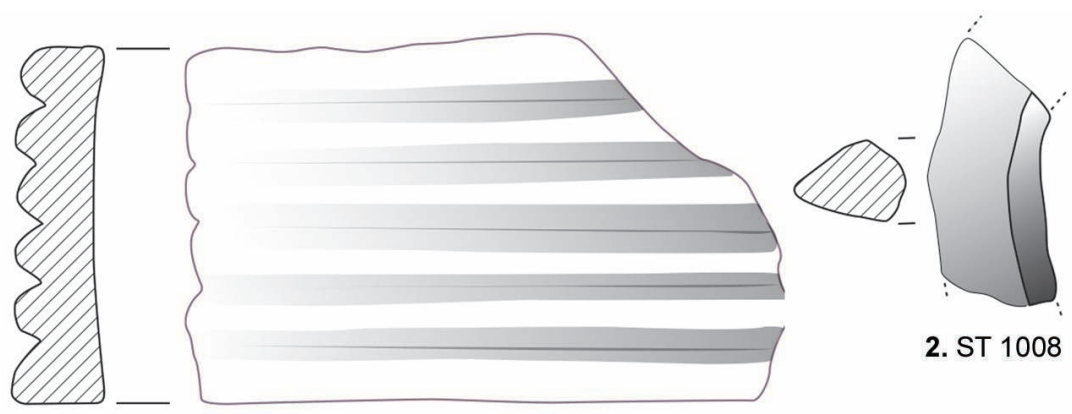

2. ST 1008

\section{ST 1008}
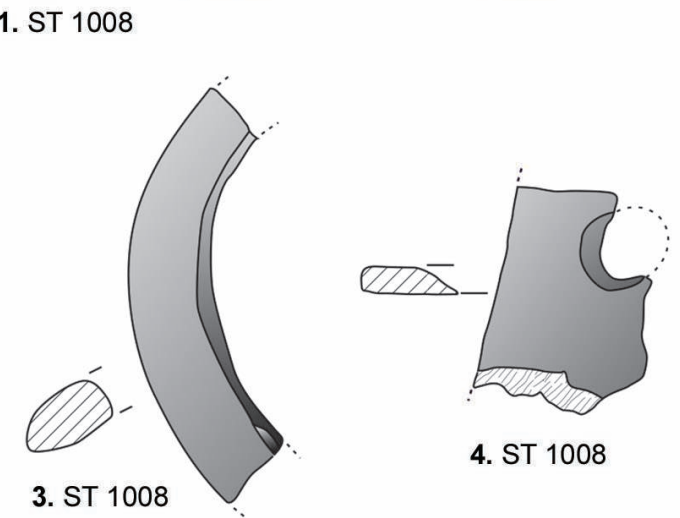

4. ST 1008

\section{ST 1008}
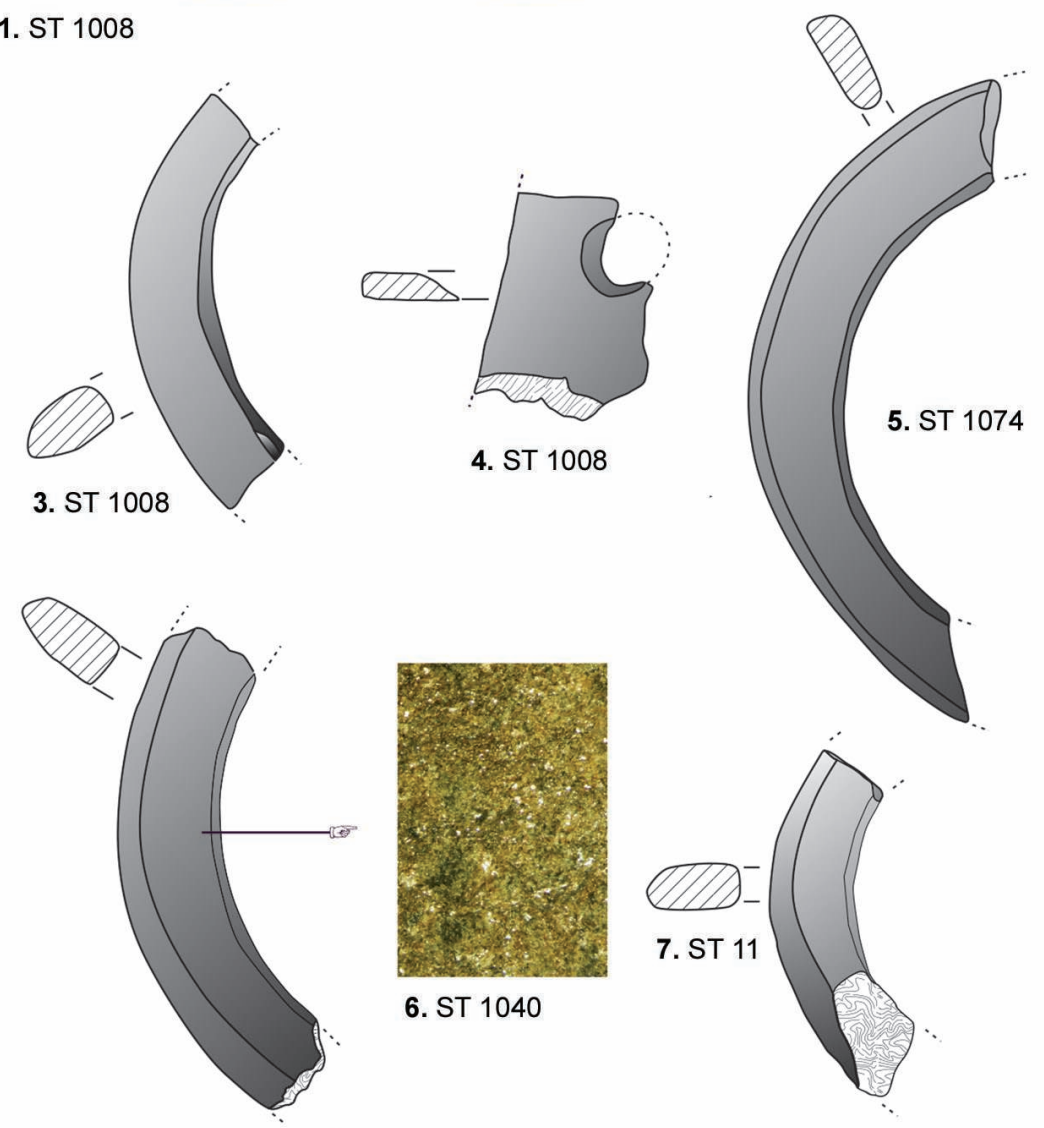

6. ST 1040
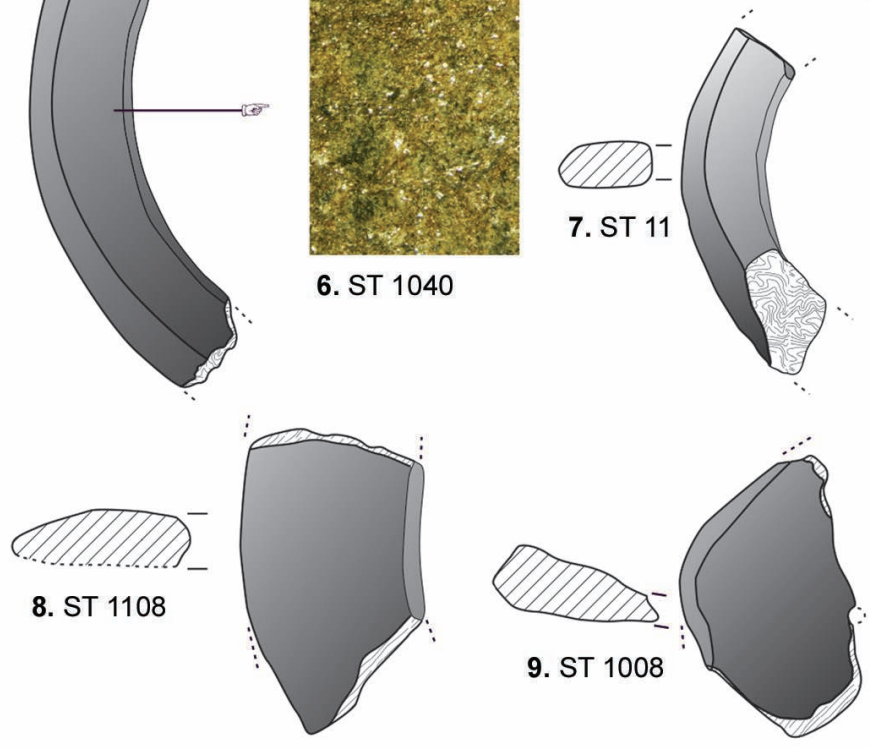
$5 \mathrm{~cm}$

Fig. 48 - Parure de bras du site de Loison-sous-Lens (DAO I. Praud, Inrap). 1. bracelet haut et rainuré en terre cuite; 2 . bracelet plat en terre cuite ; 3-5 et 7-8. bracelets en schiste ; 6 . bracelet en schiste tacheté et cliché à la binoculaire $(x 20) ; 9$. déchet de fabrication : rondelle de perforation.

Arm ornament from Loison-sous-Lens (CAD I. Praud, Inrap).

1. thick and grooved clay bracelet; 2. flat clay bracelet; 3-5. and 7-8. bracelets in schist; 6. spotted schist bracelet and stereomicroscope photo (x20); 9. manufacturing waste: perforation roundel. 


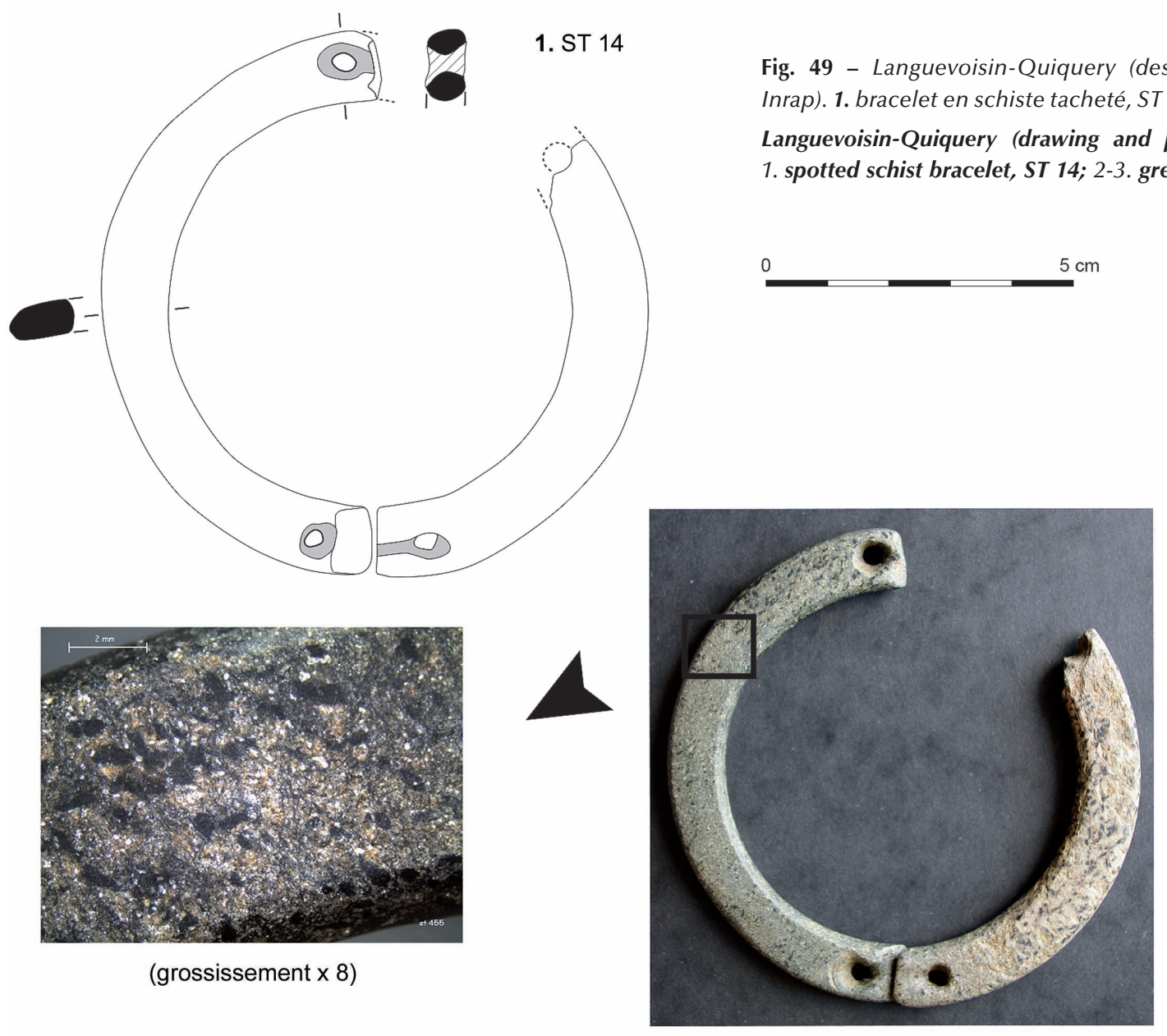

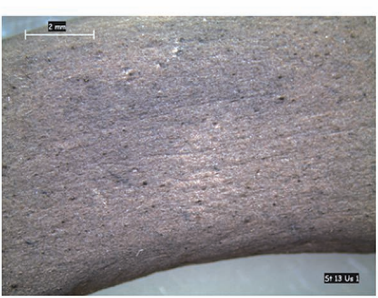

2. ST 13 US 1 (grossissement $x$ 8)

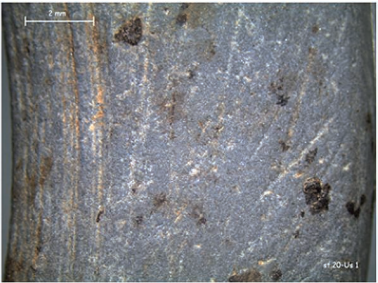

3. ST 20 (grossissement $\times 8$ )
- un schiste micacé (nombreux points brillant sous l'incidence de la lumière) qui comporte des minéraux sombres en surface répartis aléatoirement dans la matière appelés cordiérite (fig. $48, \mathrm{n}^{\circ} 6$; fig. $49, \mathrm{n}^{\circ} 1$ ) ;

- un schiste noir, feuilleté ;

- un schiste feuilleté gris tirant sur le vert, d'aspect soyeux ;

- un schiste gris-bleu en surface et noir à cœur, rayable à l'ongle - matériau assez tendre montrant en tranche un plan de schistosité marqué (fig. 49, n 2-3).

La provenance de la plupart des schistes n'est pas déterminable avec précision et les deux massifs, ardennais comme armoricain compris dans la sphère d'influence du BVSG, ont très bien pu en fournir.

Seul le schiste micacé à cordiérites, identifié pour la première fois à la fin des années 1990 (Praud et al. 2003), provient des ceintures thermo-métamorphiques entourant les massifs granitiques du nord et de l'est du Massif armoricain. Ce faciès dérive d'un métamorphisme de contact des séries schisteuses et gréseuses constituant les formations précambriennes (Briovérien) et qui les rend plus dur avec une structure interne plus homogène que d'autres formations schisteuses. L'importation de ce faciès schisteux sur les sites de Loisonsous-Lens et de Languevoisin-Quiquery localisés à proximité des sites de production de bracelet en schiste ardennais confère à ces découvertes un caractère inédit. Il permet d'en préciser le contexte chronologique par analogie avec une introduction des schistes tachetés dans les grandes vallées du Bassin parisien qui débute lors de la phase moyenne du VSG pour atteindre son apogée lors du VSG récent.

Toutefois, l'hypothèse d'une provenance ardennaise pour le reste des produits finis en schiste représenté ici ne peut 
Fig. 50 - Languevoisin-Quiquery. Traces de fabrication (dessin et clichés I. Praud, Inrap).

Languevoisin-Quiquery. Manufacturing marks (drawing and photos I. Praud, Inrap).

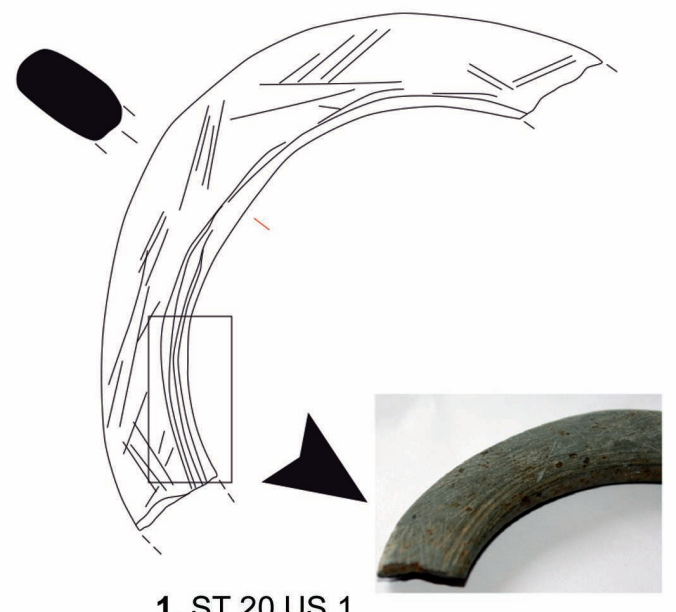

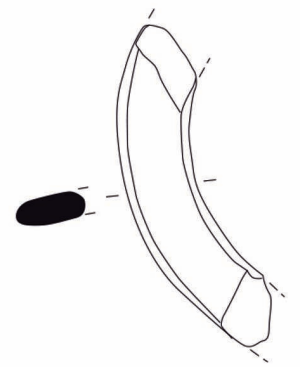

2. ST 13 US 1

\section{ST 20 US 1}

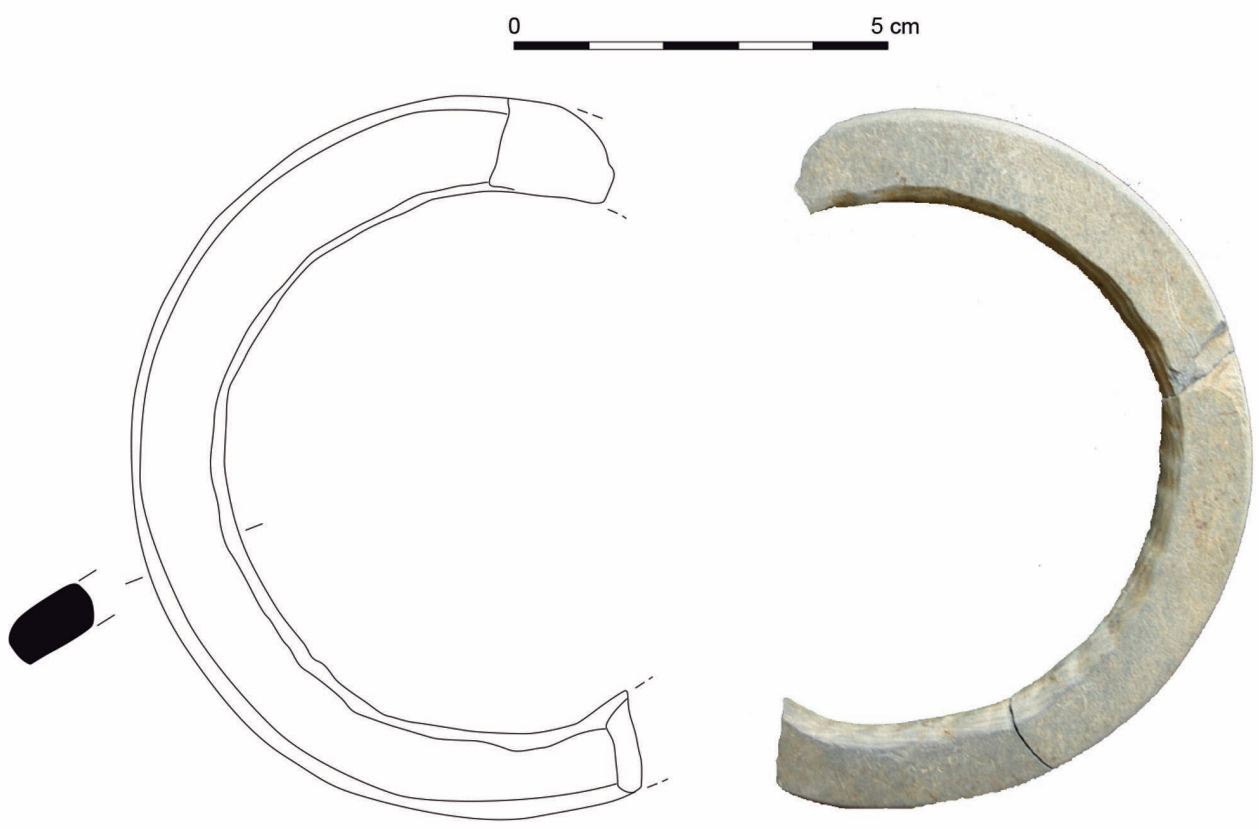

3. ST 13 US 1 1/4 NE et US 5 1/4 SW

pas être écartée car la proximité des sites producteurs belges constitue quand même un argument majeur sans que cela puisse être démontré.

\section{Fragments de Chaîne OPÉRATOIRE, Dimensions, TYPOLOGIE}

Plusieurs éléments d'observation menée sur le mobilier en schiste permettent d'illustrer une ou deux étapes de la chaîne opératoire. Il s'agit en premier lieu d'une rondelle de perforation en schiste noir d'un diamètre de $50 \mathrm{~mm}$ (fig. 48 , $\mathrm{n}^{\circ} 9$ ), qui comporte en son centre un petit trou de $2 \mathrm{~mm}$ de diamètre portant des stigmates de fracturation alors que le pourtour externe montre des traces évidentes de découpe pratiquée au silex créant un profil en «V ». Tout porte à croire que la procédure suivie par les artisans néolithiques, déjà décrite par d'autres chercheurs (Caspar et BurnezLanotte 1996, Fromont 2013), consiste à rainurer le centre du palet à l'aide d'un burin en silex calibrant le diamètre interne $\mathrm{du}$ futur bracelet qui sera ensuite régularisé par grattage et polissage. La rondelle centrale semble être détachée par pression du pouce. La présence en son centre des restes d'une perforation peut suggérer deux choses : soit une première volonté de perforation centrale à l'aide d'un foret mécanique, soit l'amorce de la fabrication avortée d'une « bague » (Caspar et Burnez-Lanotte 1996).

Enfin, quelques fragments de produits finis portent encore les stigmates des opérations de façonnage comme ces exemplaires (fig. $48, \mathrm{n}^{\circ} 3$ et 7 et fig. $50, \mathrm{n}^{\circ} 1$ et 3 ) dont les marques du découpage en segment de la partie centrale se lisent encore très bien sur le diamètre interne. Ou encore, ce bracelet en schiste tacheté très bien fini qui montre des stries parallèles très fines, réduites par un travail de surpolissage. Les extrémités des deux fragments composant ce même bracelet portent aussi des trous de réparation révélant le passage d'un lien afin de les réunir (fig. 49, n 1 ). 
Les diamètres internes des bracelets en schiste sont compris entre 50 et $70 \mathrm{~mm}$, pour des largeurs de couronne situées entre 10 et $15 \mathrm{~mm}$ - une seule atteint $24 \mathrm{~mm}$ de large. Enfin les épaisseurs sont comprises entre 4 et $6 \mathrm{~mm}$. Les sections sont de taille réduite s'inscrivant facilement dans une forme quadrangulaire plus ou moins allongée.

Les fragments de bracelet en céramique sont de deux types : deux bracelets hauts et dont la couronne extérieure est rainurée (fig. $48, \mathrm{n}^{\circ} 1$ ) et un fragment plat et lisse avec une section en ogive (fig. 48, $\mathrm{n}^{\circ} 2$ ). Les diamètres internes des bracelets rainurés et lisse sont plus grands que ceux en pierre avec des valeurs de 80 à $90 \mathrm{~mm}$. Les rainures sont réalisées sur pâte fraîche avec un profil en « V », profondes de $3 \mathrm{~mm}$ pour une largeur équivalente.

Malgré la faiblesse de son effectif, cette série offre la possibilité de discuter de la provenance des matières premières, absentes de l'environnement immédiat du site, de la forme sous laquelle ces objets circulent et enfin de la place occupée par ces sites au sein de la vaste aire géographique du BVSG (tabl. 9).

La proximité des affleurements schisteux du Brabant en Belgique exploités au cours du BVSG pour la fabrication des bracelets (Fromont 2013) et l'existence de sites d'habitat-pro- ducteur sur lesquels de nombreux déchets d'artisanat du schiste ont été reconnus, principalement à Irchonwelz « La Bonne Fortune »(Constantin et al. 1978), permettent de poser les éléments d'un débat sur la nature des relations entretenues avec ces implantations distantes de $70 \mathrm{~km}$.

Il est tout aussi instructif de s'interroger sur la chronologie des importations des produits finis en provenance du Massif armoricain au sein de l'aire de diffusion des productions ardennaises (fig. 51).

Si ces questions sont rendues plus complexes à cause des récentes avancées sur l'origine pétrographique de la série d'Irchonwelz « La Bonne Fortune », dont une partie au moins serait produite avec des schistes de la région de Mondrepuis (Aisne) dans la Haute vallée de l'Oise (Fromont et al. 2008) et du fait de l'absence de bracelet en schiste armoricain sur les sites belges, elles n'en restent pas moins intéressantes.

La présence à Loison-sous-Lens de quelques déchets de fabrication en schiste noir, probablement d'origine ardennaise, montre que certains objets sont arrivés à Loison-sous-Lens sous la forme de palets déjà régularisés mais non perforés.

Enfin, les dimensions des produits finis partagent des caractéristiques à la fois avec les séries belges notamment

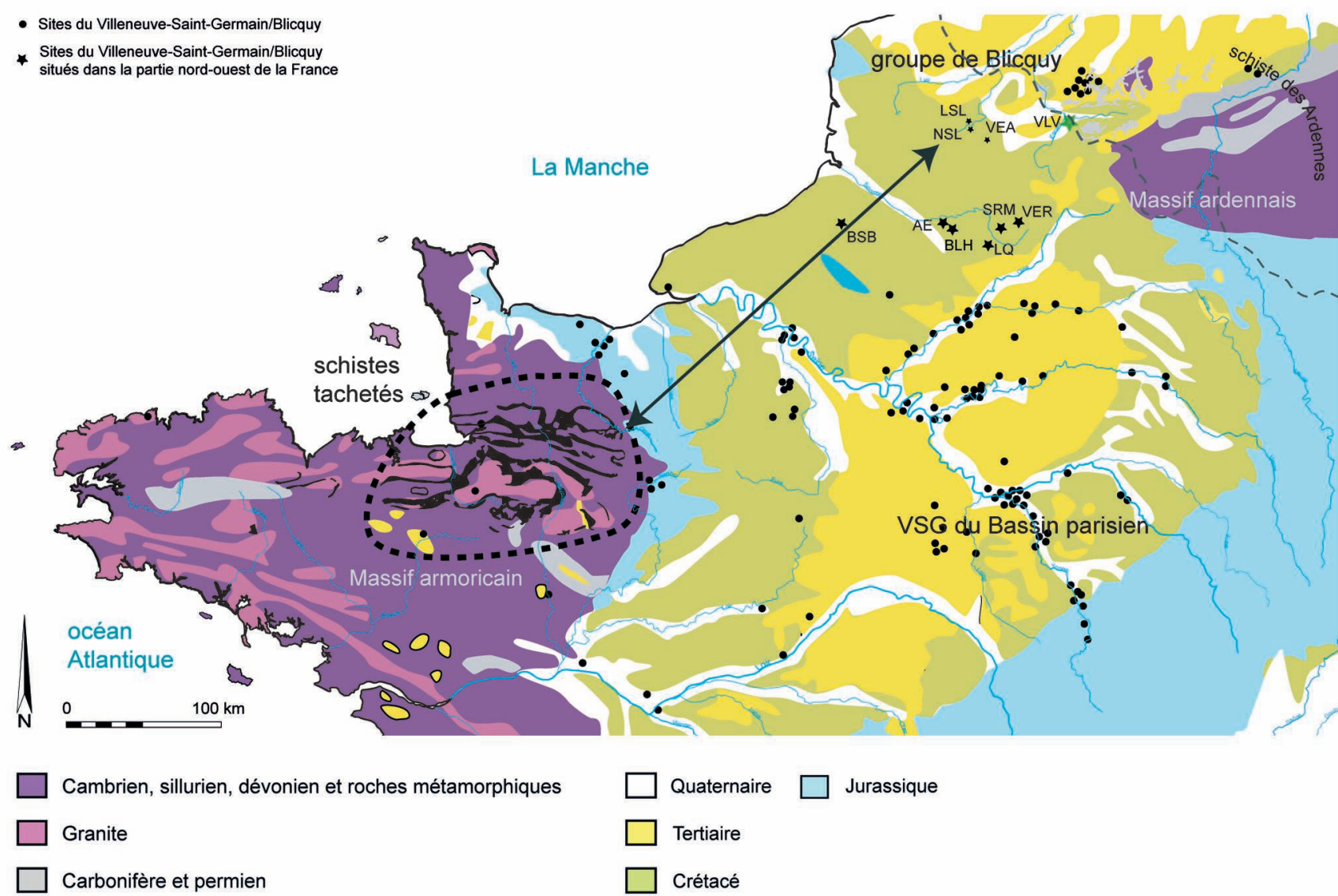

Fig. 51 - Circulation des matériaux en schiste (Carte géologique de la France. Classe de 1e D.T., t. II : Géologie, Paris, Bordas, 1972 ; DAO I. Praud, Inrap).

Circulation of schist materials (after Bordas 1972; CAD I. Praud, Inrap). 
en ce qui concerne le diamètre d'ouverture des bracelets très étroits $(62 \mathrm{~mm})$ mais aussi avec les collections normandes d'Incarville (Bostyn et al. 2003a) et celles du Bassin parisien de Rungis (ibid.).

\section{EXPLOITATION DES TERRITOIRES DANS L'ENVIRONNEMENT DES SITES}

Les modalités d'exploitation des territoires occupés seront principalement observées à travers le prisme des études carpologiques réalisées sur trois des sept sites par M.-F. Dietsch-Sellami (Loison-sous-Lens, in Praud et al. 2009a ; Languevoisin-Quiquery, in Baudry et al. 2013 ; Vitryen-Artois, in Cayol et al. 2015). À cela, il faut ajouter l'exploitation de ressources halieutiques, la distance aux ressources d'eau, les terres limoneuses à substrat crayeux et les potentiels locaux en ressources lithiques.

Les principaux résultats des études sur les macrorestes végétaux montrent que plusieurs céréales et quelques légumineuses ont été cultivées, mais aussi que les produits de la cueillette occupent une place importante dans ces corpus (tabl. 10). Sur ces trois gisements, 800 litres de sédiments ont été tamisés en colonne avec une maille de 2 et $0,5 \mathrm{~mm}$. Les refus de tamis ont livré près de 3841 macrorestes carbonisés soit une moyenne de 4,8 restes par litre. La densité est faible et montre combien la reconstitution des assemblages peut en être limitée. Le plus souvent, ces restes sont fragmentés et assez dégradés rendant ardue une détermination au niveau de l'espèce ou du genre. Les études n'ont pas cherché à lister les espèces manquantes dans ces types de contextes mais à répertorier celles qui avaient été déterminées.

Parmi ces assemblages, il y a en premier lieu les céréales qui ne dépassent jamais la moitié du nombre total des macrorestes identifiés ; Languevoisin-Quiquery et Vitry-en-Artois présentent les valeurs maximales au sein de ce corpus (entre 40 et $50 \%$ ) et c'est à Loison-sous-Lens que la part des céréales est la plus faible (23\%). Les taxons représentés sont largement dominés par les céréales à grains vêtus et en particulier l'amidonnier associé à l'engrain et l'orge. Sur les gisements de Loison-sous-Lens et de Languevoisin-Quiquery, il est intéressant de noter l'existence d'une structure au moins de type fosse qui livre de nombreux sous-produits du traitement de ces céréales (bases de glume et d'épillet, ou de rachis...) avant leur transformation en produits alimentaires. Le froment est présent sur deux gisements et on note la présence d'une légumineuse (pois) à Vitry-en-Artois et de pavot à Languevoisin-Quiquery. Enfin, sur ce dernier gisement, un reste associé à la culture du lin a été identifié.

La flore sauvage est partout représentée, souvent en rapport avec les champs emblavés accompagnant par exemple les semis d'amidonnier. On trouve le gaillet gratteron dans de fortes proportions sur le site de Languevoisin-Quiquery, cette importance pourrait être rapprochée de préparations culinaires obtenues par la cuisson des fruits de cette herbacée (DietschSellami in Baudry et al. 2013).

Les noisettes et les prunelles constituent les fruits les mieux représentés au sein du cortège des produits de la cueillette, et les coques de noisette fragmentées représentent souvent plus de la moitié des assemblages en nombre de restes. Les fruits à coque et à noyau ont en effet plus de chances d'être conservés car pour les noisettes les nombreux déchets de consommation sont rejetés dans le foyer et les prunelles sont plus digestes une fois passées au feu (Dietsch-Sellami 2007). Il faut préciser la présence, aux côtés de ces deux fruits de lisière, de restes de pommes sauvages (Malus sylvestris) parfois en quantité plus importante que les coques de noisette ainsi que de sureau noir (Sambucus nigra) à Languevoisin-Quiquery.

Dans le détail, le site de Vitry-en-Artois offre une série très dégradée, où les restes de plantes cultivées sont constitués de céréales indéterminables. Seul un caryopse mal conservé pourrait être attribué à du froment au sens large et un autre à l'amidonnier. Une semence de pois (Pisum sativum) complète ce cortège prélevé au sein de trois structures.

Tabl. 10 - Répartition en présence / absence (0/1) des restes de céréales, de légumineuses, d'oléagineuses et de fruits de lisière carbonisés par site (d'après M.-F. Dietsch-Sellami, Inrap).

Distribution of the carbonised macro-remains by site (after M.-F. Dietsch-Sellami, Inrap).

\begin{tabular}{|c|c|c|c|c|c|c|c|}
\hline Sites & Litres & Restes carbonisés & Moy/litres & Nbr taxons & Céréales & Gr & Céréales/carpo géné \\
\hline LSL & 186 & 786 & 4,23 & 12 & 183 & 127 & $23 \%$ \\
Vitry & 289 & 85 & 0,29 & 13 & 37 & 37 & $43 \%$ \\
LQ & 326 & 2970 & 9,11 & 19 & 1300 & $43,77 \%$ \\
\hline Total & 801 & 3841 & 4,80 & 44 & 1520 & 164 & $40 \%$ \\
\hline
\end{tabular}

\begin{tabular}{|c|c|c|c|c|c|c|c|c|c|c|}
\hline Sites & $\begin{array}{l}\text { Amidonnier } \\
\text { (Triticum } \\
\text { dicoccum) }\end{array}$ & $\begin{array}{c}\text { Engrain } \\
\text { (Triticum } \\
\text { monococcum) }\end{array}$ & $\begin{array}{c}\text { Orge polystique } \\
\text { vêtue (Hordeum } \\
\text { vulgare) }\end{array}$ & $\begin{array}{c}\text { Froment } \\
\text { (Triticum } \\
\text { aestivum I.s.) }\end{array}$ & $\begin{array}{c}\text { Pavot } \\
\text { (Papaver } \\
\text { somniferum) }\end{array}$ & $\begin{array}{l}\text { Légumineuses } \\
\text { (Pisum sativum) }\end{array}$ & $\begin{array}{l}\text { Noisetier } \\
\text { (Corylus } \\
\text { avellana) }\end{array}$ & $\begin{array}{c}\text { Pommier } \\
\text { sauvage (Malus } \\
\text { sylvestris) }\end{array}$ & $\begin{array}{l}\text { Prunellier } \\
\text { (Prunus } \\
\text { spinosa) }\end{array}$ & $\begin{array}{c}\text { Sureau noir } \\
\text { (Sambucus } \\
\text { nigra) }\end{array}$ \\
\hline LSL & 1 & 1 & 1 & 1 & 0 & 0 & 1 & 0 & 1 & 0 \\
\hline VEA & 1 & 0 & 0 & 1 & 0 & 1 & 1 & 0 & 1 & 1 \\
\hline LQ & 1 & 1 & 1 & 1 & 1 & 0 & 1 & 1 & 1 & 1 \\
\hline
\end{tabular}


Quatre céréales ont été identifiées sur le site de Loisonsous-Lens : l'amidonnier (Triticum dicoccum) et l'engrain (Triticum monococcum) représentés principalement par des restes de décorticage, le froment (Triticum aestivum/turgidum/ durum) représenté autant par les grains que par des fragments de rachis, et l'orge polystique vêtue uniquement signalée par ses grains. La fréquence du froment est équivalente à celle de l'amidonnier dans les neuf structures échantillonnées.

À Languevoisin, quatre structures ont livré des céréales à grains vêtus (amidonnier, engrain, orge). Le froment n'est pas absent de ce corpus mais moins fréquent qu'à Loison-sousLens. Une semence de pavot (Papaver somniferum) attestée dans l'une de ces structures complète la série.

La fosse $36 \mathrm{du}$ site de Vitry-en-Artois (fosse dite en « Y ») mérite que l'on s'y attarde un peu car elle livre quatre taxons d'herbacées sauvages et de fruits en faible nombre certes, mais jamais identifiés auparavant dans des structures du Néolithique ancien en contexte terrestre. La renoncule flammette, le plantain lancéolé, le souchet des marais et des fruits de tilleul sont associés dans les mêmes couches de remplissage de cette fosse. Or, trois d'entre elles sont caractéristiques des milieux humides et témoignent d'un sédiment gorgé d'eau tandis que les fruits de tilleul ont été rapportés intentionnellement. Ces observations pourraient permettre d'envisager une fonction de rouissage pour cette structure à partir de l'association entre plantes d'eau et tilleul dont le liber a pu être transformé en vue de fabriquer des étoffes cordées. Cette hypothèse n'a cependant pas été confirmée par les études géomorphologiques parce que les sédiments ne présentent pas de traces de stagnation d'eau et l'encaissant n'a pu être affecté par le battement de la nappe phréatique (Cayol et al. 2015). Enfin, toujours au sein de ce même remplissage, plusieurs restes de poissons de rivière pêchés et consommés (seize individus) ont été rejetés.

Une seconde fosse en " $\mathrm{Y}$ » découverte à LanguevoisinQuiquery ne confirme pas plus une fonction liée au rouissage même si un parasite de la culture du lin y a été identifié. D'autres hypothèses pour ces types de fosse sont régulièrement évoquées comme, par exemple, les fosses-pièges pour la chasse d'animaux sauvages (Achard-Corompt et al. 2011).

Tous les gisements sont implantés sur des limons de plateau plus ou moins épais. Ces terres possèdent les qualités agricoles recherchées par les premiers colons néolithiques et la présence systématique de céréales en atteste. Les assemblages de carporestes s'inscrivent dans la continuité de ceux observés pour le Rubané : céréales à grains vêtus majoritaires, pavot et pois (Dietsch-Sellami 2007). L'existence de restes de blé nu associés aux blés vêtus, amidonnier et engrain, montre que des changements dans les pratiques agricoles sont déjà perceptibles ici. La présence de froment, dont la farine est panifiable, dans les corpus annonce les transformations à venir, car cette céréale deviendra majoritaire au Néolithique moyen sur ces mêmes terres agricoles (ibid.).

En raison de la nature du sous-sol, composé de limons décarbonatés, les restes osseux ne sont malheureusement pas conservés sur les sites étudiés ici, pas plus que l'industrie en os. Cependant, l'analyse fonctionnelle des outils en silex ou la présence des armatures de flèches met en évidence l'existence d'un apport carné, domestique ou sauvage, dans le régime alimentaire des populations.

La proximité de l'eau semble être aussi un critère de choix commun à tous les sites et la présence de poissons sur l'un des gisements témoigne de l'intérêt que ces populations ont pu porter d'un point de vue alimentaire aux ressources halieutiques, phénomène déjà observé sur le site de Trosly-Breuil (BVSG moyen ; Clavel 2009) ou celui de Longueil-SainteMarie « Le Barrage » (Bostyn et al. 2015).

\section{SYNTHÈSE}

\section{CHRONOLOGIE DES OCCUPATIONS}

Trois gisements ont bénéficié de datations radiocarbone (tabl. 11). Les résultats bruts montrent que la majorité des structures ( 8 sur 10) se répartit sur un segment chronologique correspondant peu ou prou à la durée admise du BVSG, soit 300 ans (5000/4950-4700/4650 av. J.-C. ; Dubouloz 2003). Les recoupements des dates entre les charbons de bois, dont les essences n'ont pas été déterminées en amont de la datation, issus de Vitry-en-Artois, Languevoisin-Quiquery et les coquilles de noisette provenant du site de Loison-sous-Lens indiquent que les échantillons et les résultats sont corrects (fig. 52).

Toutefois, deux dates dépassent le cadre chronologique du BVSG : la première réalisée sur un os long (fémur) de la sépulture de Loison-sous-Lens (SP 1083) est datée dans un intervalle à deux sigma entre 4534-4335 cal. BC tandis que celle issue d'un charbon de bois de la structure 9 de LanguevoisinQuiquery se trouve comprise entre 3264 et 2929 cal. BC.

Le mobilier déposé au sein de la sépulture est un micro-vase en forme de bouteille qui comme nous l'avons déjà souligné est proche des productions reconnues sur des sites BVSG alors que la mesure isotopique indiquerait plutôt une datation de la culture de Cerny. Plusieurs éléments nous amènent cependant à considérer cette inhumation comme appartenant au BVSG : la position du haut du corps en décubitus dorsal, les membres inférieurs repliés sur le côté droit, la tête à l'est et enfin un corps associé à un vase non décoré déposé au niveau du genou constituent à nos yeux des arguments suffisants. Au moment de trancher, nous accordons donc ici plus de poids à la position du corps et au mobilier archéologique associé qu'à celui du résultat de la datation absolue faite sur os qui pourrait être lié à un problème de conservation du collagène. La seconde structure (une fosse en «V $»$ ) ne livre que quelques tessons peu caractéristiques, elle restera attribuable à une séquence du Néolithique récent/final.

Trois fosses de rejets détritiques ont été datées par la méthode du radiocarbone sur le site de Loison-sous-Lens (fig. 53 et tabl. 11). Il s'agit pour la partie nord du décapage des structures $1008-7^{\mathrm{e}}$ passe (fond de la structure : $6085 \pm 35 \mathrm{BP}$ ) et $1008-3^{\mathrm{e}}$ passe $(6040 \pm 35 \mathrm{BP})$, qui est la fosse la plus riche en mobilier et la plus profonde du site, ainsi que de la structure 11 $(6040 \pm 35$ BP) qui se localise à quelques mètres, et enfin pour le secteur sud de la fosse $1137(6000 \pm 35 \mathrm{BP})$. 
Tabl. 11 - Résultats des mesures isotopiques du carbone 14 (I. Praud, Inrap).

Results of the Carbon 14 isotopic measurements (I. Praud, Inrap).

\begin{tabular}{|c|c|c|c|c|c|c|c|}
\hline Site & Échantillon & Code laboratoire & BP & Cal. BC_1 sigma & Cal. BC_2 sigma & Cal. BC médiane & Méthode \\
\hline LSL_st 1008_p7 & coq. noisette & GrA-38102 & $6085 \pm 35$ & $5044-4947$ & $5206-4851$ & 5000 & AMS \\
\hline LSL_st 1008_p3 & coq. noisette & GrA-38101 & $6040 \pm 35$ & $4993-4856$ & $5031-4841$ & 4940 & AMS \\
\hline LSL_st 11 & coq. noisette & GrA-38099 & $6040 \pm 35$ & $4993-4856$ & $5031-4841$ & 4940 & AMS \\
\hline LSL_st 1137 & coq. noisette & GrA-38103 & $6000 \pm 35$ & $4938-4842$ & $4986-4799$ & 4890 & AMS \\
\hline LSL_sep 1083 & fémur & GrN-31128 & $5580 \pm 60$ & $4457-4357$ & $4534-4335$ & 4417 & traditionnelle \\
\hline Vitry_st 36 & charbons de bois & Poz-44548 & $6025 \pm 35$ & $4979-4849$ & $5016-4829$ & 4919 & traditionnelle \\
\hline LQ_st 9 & charbons de bois & Poz & $4420 \pm 20$ & $3092-3021$ & $3264-2929$ & 3054 & AMS \\
\hline LQ_st 20 & os brûlé & Poz-44412 & $6090 \pm 40$ & $5056-4942$ & $5207-4854$ & 5008 & AMS \\
\hline LQ_st 13 & charbons de bois & KIA-46216 & $6000 \pm 35$ & $4938-4842$ & $4986-4799$ & 4890 & AMS \\
\hline LQ_st 14 & charbons de bois & Poz-44549 & $5840 \pm 40$ & $4784-4687$ & $4826-4602$ & 4720 & AMS \\
\hline VLV_D1 & tourbe & Erl-11736 & $7310 \pm 59$ & $6226-6097$ & $6354-6048$ & 6163 & AMS \\
\hline VLV_D2 & tourbe & Erl-11735 & $7043 \pm 63$ & $5998-5851$ & $6031-5772$ & 5925 & AMS \\
\hline VLV_D3 & tourbe & Erl-11734 & $6901 \pm 49$ & $5837-5731$ & $5896-5675$ & 5785 & AMS \\
\hline VLV_D4 & tourbe & Erl-11733 & $6297 \pm 43$ & $5314-5226$ & $5375-5082$ & 5274 & AMS \\
\hline VLV_D5 & tourbe & Erl-11732 & $6247 \pm 43$ & $5307-5083$ & $5316-5066$ & 5235 & AMS \\
\hline VLV_D6 & tourbe & Erl-11731 & $6076 \pm 43$ & $5052-4933$ & $5206-4844$ & 4988 & AMS \\
\hline VLV_D7 & bois, cernes externes & Erl-11729 & $6026 \pm 42$ & $4985-4849$ & $5030-4801$ & 4920 & AMS \\
\hline VLV_D8 & tourbe & Erl-11730 & $5422 \pm 43$ & $4334-4257$ & $4355-4079$ & 4285 & AMS \\
\hline
\end{tabular}

OxCal v4.3.2 Bronk Ramsey (2017); r:5 IntCal13 atmospheric curve (Reimer et al. 2013)

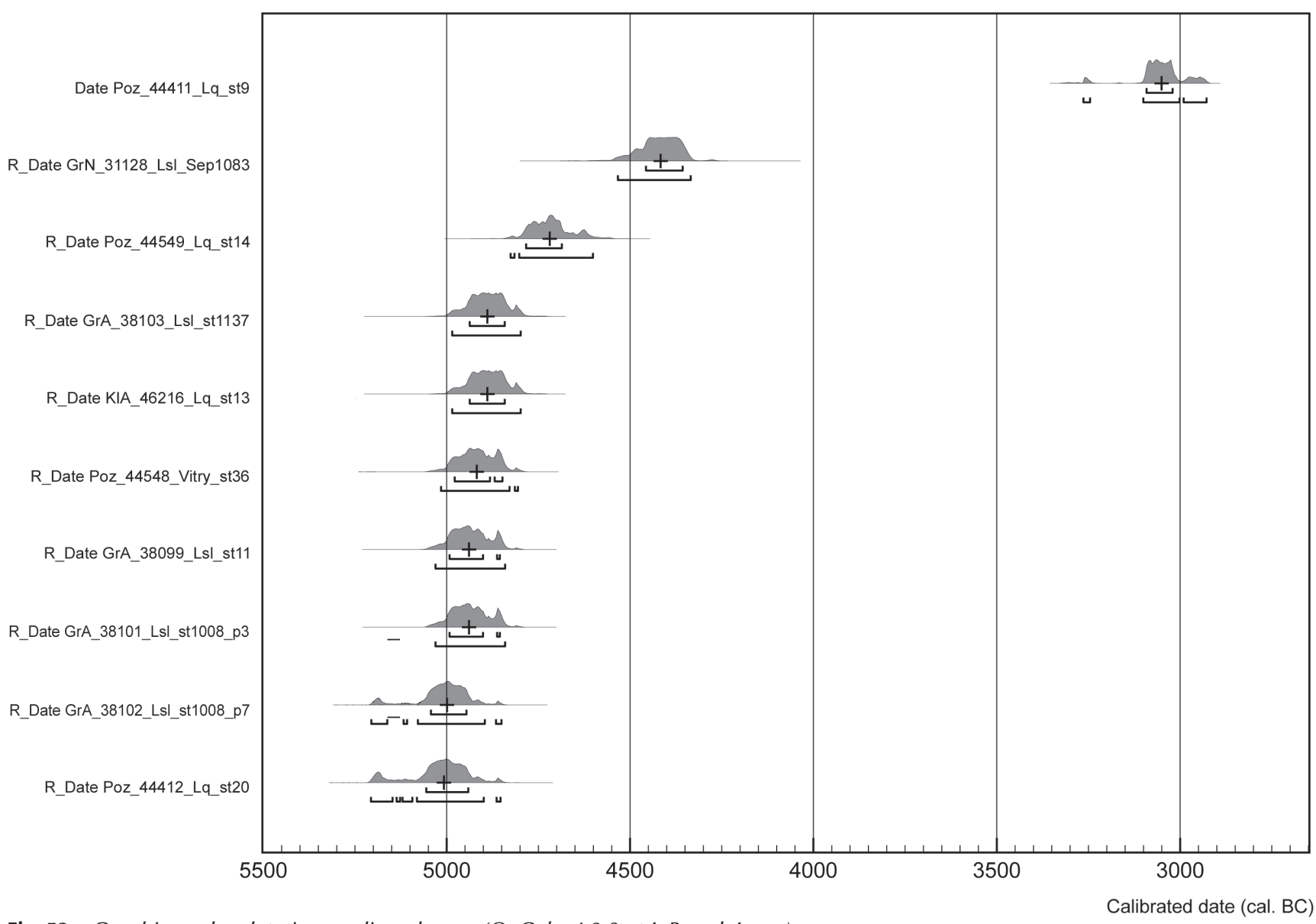

Fig. 52 - Graphique des datations radiocarbones (OxCal. v4.3.2 et I. Praud, Inrap).

Graph of radiocarbon dates (OxCal. v4.3.2 and I. Praud, Inrap). 
OxCal v4.3.2 Bronk Ramsey (2017); r:5 IntCal13 atmospheric curve (Reimer et al. 2013)

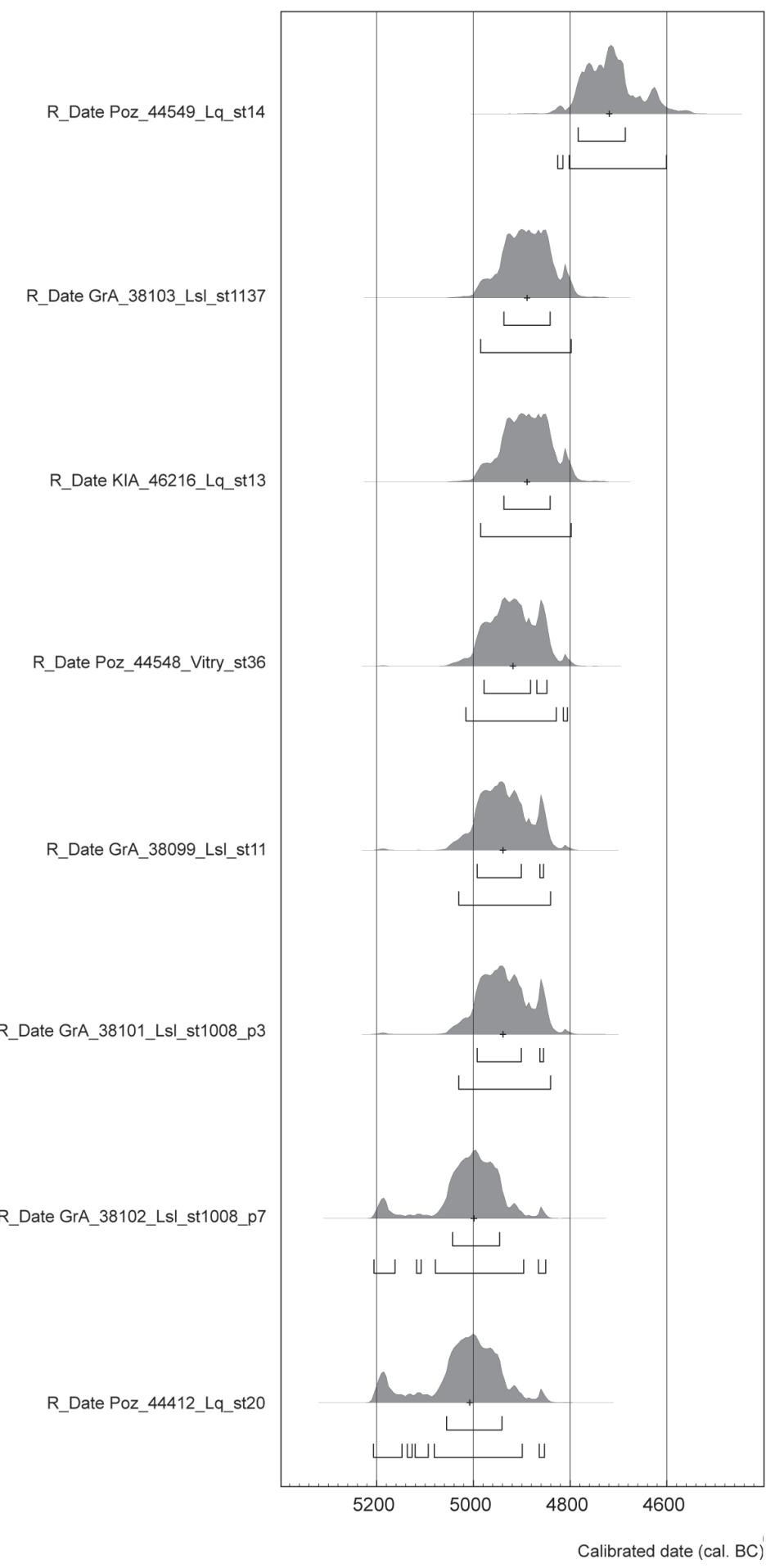

Fig. 53 - Graphique des datations radiocarbones et zones de recouvrement des résultats (OxCal. v4.3.2 et I. Praud, Inrap).

Graph of radiocarbon dates showing zones where results overlap (OxCal. v4.3.2 and I. Praud, Inrap).
Pour toutes les raisons soulevées par de nombreux chercheurs, nous avons été extrêmement prudents sur les contextes des éléments soumis à la datation en privilégiant avant tout les échantillons à courte durée de vie comme les coquilles de noisette carbonisées. Elles ont été prélevées au sein de lots de grains de céréales et datées de la même manière par accélérateur (Whittle 1990). Les résultats obtenus sur la fosse de rejet détritique 1008 respectent non seulement la succession stratigraphique entre la $7^{\mathrm{e}}$ et la $3^{\mathrm{e}}$ passe, mais présentent aussi une bonne cohérence chronologique entre les structures. En effet, les trois dates provenant d'une possible unité d'habitat (ST 1008 et 11) se distribuent au sein d'un intervalle compris entre 5044 et 4841 cal. BC à 1 sigma. La combinaison des trois dates confirme l'attribution à une phase relativement ancienne du BVSG (fig. 54) comprise à deux sigma entre 5025 et 4855 cal. BC.

Les marges d'incertitude des dates réalisées sur les restes carpologiques livrés par ces fosses de rejet n'excèdent pas \pm 35 années. Le recoupement des fourchettes calibrées conforte la durée de l'occupation de Loison-sous-Lens dans un intervalle compris entre 5000 et 4890 cal. BC.

La courbe de calibration est très favorable pour cette période et les résultats en années réelles peuvent être considérés comme fiables (Oberlin 2003). On observe une datation ancienne du point de vue des mesures radiocarbone pour cette occupation qui la rapproche des contextes culturels les plus anciens des manifestations du BVSG succédant à la fin du Rubané dans le Bassin parisien et en Belgique (Dubouloz 2003). Il est complexe d'être beaucoup plus précis avec les calculs de probabilité. Confronté au mobilier archéologique, il n'y a pas d'incohérence à maintenir une datation de cet ensemble dans la phase d'un BVSG classique soit autour de 4900-4850 cal. BC même si nous nous trouvons dans la partie haute de la fourchette.

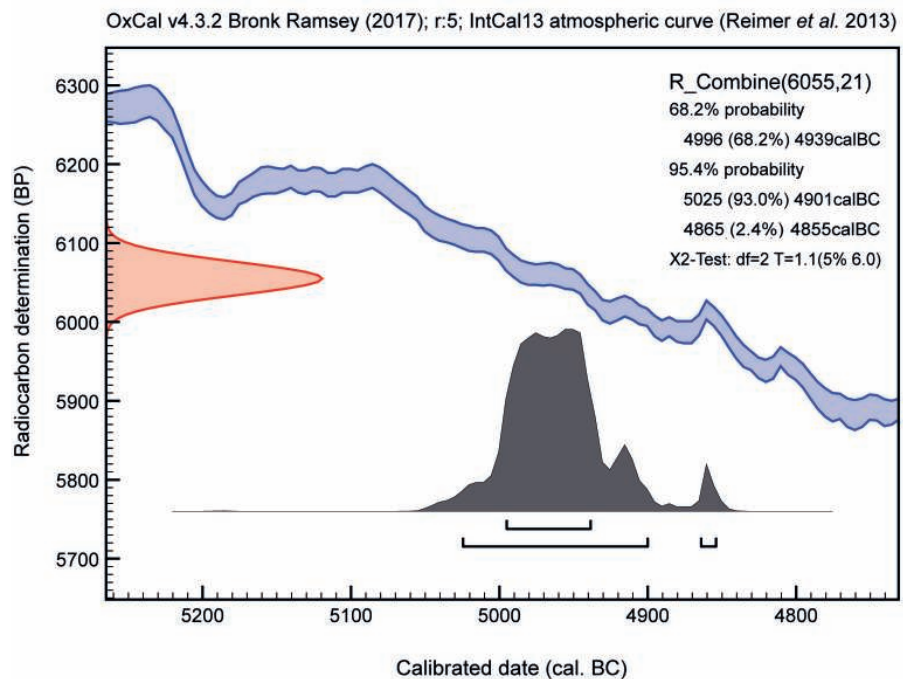

Fig. 54 - Combinaison des trois datations de l'unité d'habitat de Loison-sous-Lens comprenant les structures 1008 et 11 (grâce à la fonction " $R_{-}$Combine " du logiciel OxCal v4.3.2).

Combination of three dates from the settlement unit of Loison-sous-Lens comprising structures 1008 and 11 (using the "R_Combine" function with the OxCal software v4.3.2). 
Les datations sur le site de Languevoisin-Quiquery montrent les mêmes probabilités de recouvrement mais avec des bornes plus récentes comprises entre 4950 et 4740 cal. BC (fig. 53). Dans le détail, on remarque une date ancienne (ST 20) qui peut être liée à l'échantillon daté ici un os calciné. L'échange entre l'os (non déterminé ici) et les autres sources de carbone comme le bois durant la crémation peuvent altérer la date obtenue en transférant un « effet vieux bois » difficile à détecter (Zazzo et al. 2009). Les inconnues sont trop nombreuses pour considérer cette date comme fiable. La date de la structure 14 paraît complètement décalée par rapport au reste des résultats. Ces données rapportées au mobilier archéologique attribuable à la phase récente/finale du BVSG, montrent une fois de plus que les mesures isotopiques sur des échantillons non déterminés (os comme charbon de bois) livrent des résultats difficiles à mettre en perspective avec un phasage chrono-culturel. En l'occurrence, si nous devions nous conformer à des ensembles de dates plus étendus nous aurions tendance à proposer une date moyenne de la phase récente/finale du BVSG autour de 4750 cal. BC.

Un autre scénario est possible qui verrait se succéder plusieurs phases sur un même gisement, mais le mobilier ne permet pas une analyse aussi fine. Les blocs définis ainsi semblent plus cohérents d'un point de vue archéologique. Ainsi, les sites découverts dans ce secteur géographique appartiendraient majoritairement à la phase récente-finale du BVSG pour autant que l'on puisse en juger à partir de l'analyse des mobiliers et des datations isotopiques. À ce jour, le gisement de Loison-sous-Lens serait le plus ancien dans ce mouvement d'extension territoriale des communautés agro-pastorales, qui serait dès lors attribuable à la phase moyenne du BVSG.

\section{STATUT DES SITES ET RÉSEAUX D'ÉCHANGES}

Comme les analyses précédentes ont pu le montrer, les données réunies sur chacun des sites fouillés ne sont pas strictement comparables, les études les plus abouties ayant été menées sur deux gisements attribuables à des phases différentes du BVSG - Loison-sous-Lens pour la phase moyenne et Languevoisin-Quiquery pour la phase finale. Les données architecturales sont trop fragmentaires pour pouvoir utiliser ces critères dans une discussion approfondie sur une possible différenciation entre les sites. Néanmoins, la réflexion menée sur la morphologie des fosses et leur agencement dans l'espace a permis de proposer une différenciation entre les sites d'habitat et les autres types de sites correspondant sans doute à des occupations à vocations plus spécifiques et artisanales. Rappelons également qu'une partie des informations nous manque en raison de l'absence de faune et donc aussi d'industrie osseuse. Cependant, les études menées sur les mobiliers présents amènent des informations indirectes sur les activités quotidiennes et nous permettent quand même d'aborder les questions du statut des sites et d'organisation économique à l'échelle au moins régionale.

\section{Des habitATS PÉRENNES D'AGRO-PASTEURS}

Au moins trois indicateurs plaident pour une pratique intense des activités de production et de consommation agricoles.

Tout d'abord, le nombre important d'éléments de faucille, comme sur le site de Loison-sous-Lens dédiés à la récolte de céréales, plaide pour une pratique intensive de l'agriculture.

Ces données s'accordent bien avec la mise en évidence de plusieurs céréales (amidonnier, engrain, orge vêtu, froment), par ailleurs associées à des restes de fruits provenant de la cueillette (noisette et prunelle). La flore sauvage est plutôt bien représentée dans ces séries. Languevoisin-Quiquery se distingue par la présence en plus de nombreux restes de pomme sauvage, de sureau noir et de gaillet grateron.

En outre, à Loison-sous-Lens, la présence en quantité exceptionnellement importante de meules entières de différents types, dont les molettes associées ont été principalement utilisées pour la mouture des céréales d'après l'analyse tracéologique, traduit des épisodes de traitement des céréales avant consommation durablement inscrits dans les pratiques culinaires des habitants du site. On peut écarter l'idée d'un site producteur de meules et molettes en grès puisque les restes de fabrication sont peu nombreux. S'il reste difficile de trancher sur le statut des objets découverts en contexte détritique (dépôts démantelés, mise en réserve ou abandon), le nombre élevé de meules entières, en comparaison avec les sites du Bassin parisien où elles sont le plus souvent fracturées, confirme l'importance des activités de production et de consommation agricoles sur ce site d'habitat.

\section{Des ACTIVITÉS ARTISANALES TRÈs ORIENTÉES VERS L'EXPLOITATION DU MILIEU VÉGÉTAL}

L'approche techno-fonctionnelle des outillages en pierre met en évidence que l'outillage en silex est en majorité dirigé vers l'exploitation du milieu végétal. Il est utilisé pour exploiter les espaces naturels et / ou le binage des cultures (tranchet), il est sollicité pour la coupe et le raclage des végétaux (burin, denticulés) notamment pour la récolte des céréales (armature de faucille) et le teillage de fibres végétales (burin exclusivement). Cette activité dominante sur l'exploitation et la transformation des végétaux est amplement confirmée par la présence importante dans l'outillage de burins et de faucilles confectionnés sur lame mais aussi ici sur éclat. Il faut remarquer l'absence de tranchets sur le site de Languevoisin-Quiquery alors qu'il est représenté sur trois autres gisements (Loison-sous-Lens, Boves et Vitryen-Artois). Le travail du bois est moins bien représenté en général, il concerne les activités de raclage, de rabotage et de coupe (denticulé, débris, éclat retouché et grattoir herminette).

La chasse et la transformation des produits issus de cette activité et de l'élevage (os, peau et découpe-raclage) mettent aussi à contribution l'outillage en silex mais dans des proportions plus faibles, ce qui se reflète dans la faible proportion dans les corpus de grattoirs sur éclat aussi utilisés pour le 
travail de la peau (Allard et al. 2004), contrairement aux séries du Bassin parisien où il est dominant (Allard et Bostyn 2006).

L'exploitation et la transformation du milieu végétal sont largement prépondérantes dans les activités artisanales de notre région d'étude, et si on ajoute la présence du lin à LanguevoisinQuiquery, on peut se demander, si au-delà d'une vocation économique particulière, ces observations ne refléteraient pas une évolution croissante à partir de la fin du BVSG de la part du textile au détriment du travail de la peau (vêtements, ligatures, par exemple). Multiplier les analyses fonctionnelles dans l'aire d'étude concernée ici, mais aussi plus largement à l'échelle du Bassin parisien, apporterait probablement des informations complémentaires qui pourraient permettre de développer ces problématiques, même si les premiers résultats dont nous disposons aujourd'hui montrent une place prépondérante du grattoir dans les assemblages utilisés pour le travail des peaux (Allard et al. 2004).

\section{Des ASSEMblages LithiQues PARTICULIERS}

Des différences dans l'acquisition des matières premières, dans les savoir-faire mis en œuvre pour les transformer et dans la composition des mobiliers ont été soulignées. Si la production d'un outillage en silex sur éclat à partir de matériaux locaux est observée sur tous les sites étudiés, seul le site du BVSG moyen de Loison-sous-Lens rassemble les restes de débitage d'une production laminaire en silex secondaire réalisée sur place. Ailleurs, les lames sont importées essentiellement sous la forme de produits finis. Cette situation est inédite puisque les multiples études lithiques ont pu montrer que les productions laminaires sur les matériaux locaux étaient toujours réalisées sur place (Bostyn 1994, Bostyn et Denis 2016). Ceci renforce le statut particulier des sites autres que Loison-sous-Lens, pour lesquels nous disposons d'informations significatives et qui ne s'intègrent pas à ce qu'il convient d'appeler les sites d'habitat, ils sont bien des lieux d'activités temporaires.

Cependant, pour le BVSG final, la composition de la série de Boves, même si elle n'est que partielle, nous amène à nous interroger sur l'éventualité d'une perte de savoir-faire pour la production laminaire qui serait alors synonyme d'une plus grande dépendance entre sites pour l'acquisition de ces supports d'outils et qui pourrait aussi être un élément d'explication à la baisse importante de la part des productions laminaires au sein du BVSG.

\section{UNE INTÉGRATION DANS LES RÉSEAUX D'ÉCHANGE}

La présence de matériaux non locaux a été observée aussi bien dans l'industrie en silex que dans la parure. Les travaux de synthèse sur les industries en silex ont largement démontré la place prépondérante du silex bartonien du Bassin parisien dans les réseaux de circulation existant dans le quart nord-ouest de la France et en Belgique, pouvant ainsi être considéré comme un marqueur social et identitaire essentiel pour la cohésion de ce groupe culturel sur un vaste territoire s'étendant du Bassin parisien au Hainaut belge et de la Basse-Normandie à la partie amont de la vallée de la Seine (Bostyn 1994 et 2008, Bostyn et Denis 2016, Charraud 2013, Denis 2014a, Bostyn et al. à paraître a). Les outils réalisés sur ces produits investis techniquement sont ici aussi ceux qui sont caractéristiques de cette culture (burins, armature de faucille, armature) renforçant ce caractère identitaire (Bostyn 2008). Cette diffusion intense est donc une nouvelle fois confirmée ici, en particulier par la présence de lames dans la vallée de la Somme à Étouvie, loin des axes majeurs de circulation qui se dessinent le long des vallées principales.

Par contre, la situation est bien différente pour le silex de Ghlin qui n'est attesté qu'à Vermand, ce qui confirme le faible attrait des habitants du Bassin parisien pour ces produits, mais conforte la Haute vallée de l'Oise comme jalon entre les régions. La circulation du silex de Ghlin reste donc largement tournée vers les sites de Hesbaye (Denis 2014b). L'absence de ce silex à Loison-sous-Lens pose alors question, compte tenu de sa proximité géographique avec les sites du Hainaut belge, mais elle est peut-être à mettre sur le compte de cette forte implication dans l'économie des sites de Hesbaye.

Les principaux gisements livrent également des bracelets, marqueurs identitaires du BVSG, qu'ils soient en pierre ou en terre cuite. Les déterminations macroscopiques ont montré sur les sites de Loison-sous-Lens et de Languevoisin la présence de matériaux originaires à la fois du Massif ardennais et du Massif armoricain, indiquant leur circulation précoce sur de très longues distances dépassant largement les grandes vallées du Bassin parisien (Praud et al. 2003) pour atteindre également la Belgique (Fromont 2003).

Cette circulation de différentes matières premières montre donc que notre secteur d'étude est parfaitement intégré au sein du territoire BVSG, et est en relation indirecte avec des sites à la fois proches et plus lointains.

\section{APPORT DES SITES À LA COMPRÉHENSION DU PROCESSUS DE NÉOLITHISATION DES MARGES NORD-OCCIDENTALES DE LA FRANCE}

\section{Des connaissances renouvelées}

Au-delà des apports sur la question des statuts des sites, plusieurs autres données apportent une vision renouvelée de la culture de BVSG.

La présence du froment arrivant juste derrière l'amidonnier dans le spectre carpologique de Loison-sous-Lens alors que ce blé nu n'était identifié qu'au BVSG récent dans le Bassin parisien (Dietsch-Sellami 2007), constitue un premier point important.

L'introduction de cette nouvelle céréale entraîne de nouvelles pratiques car elle est plus exigeante au niveau climatique, n'offre pas un rendement supérieur aux céréales à grains vêtus et serait même plus fragile globalement que l'amidonnier et l'engrain (Bakels 2008). Ce changement majeur pourrait relever d'influences extérieures (plante cultivée depuis les débuts du Néolithique ancien dans le Sud de la France), correspondre à des besoins alimentaires nouveaux (fabrication de galette de pain) et pourrait être à l'origine de transformations dans d'autres secteurs d'activité. 
Le développement de cette nouvelle céréale intervient en même temps qu'une autre innovation précoce dans le domaine des techniques : le tranchet en silex. Cette relation n'est pas anodine et signe un changement dans les activités agricoles (entretien des champs cultivés et utilisation du tranchet pour le binage) pour cultiver cette céréale à grain nu plus fragile que les céréales rustiques. La culture du froment et la fabrication des tranchets n'étaient, jusque-là, connues que dans la phase récente du BVSG dans le Bassin parisien. Or, l'attribution chronologique du site de Loisonsous-Lens à l'étape moyenne, sur la base de la céramique et des dates radiocarbone, tendrait à en vieillir leur apparition. Cette association entre éléments récents dans l'industrie du silex (armatures tranchantes, tranchets) et anciens dans la céramique (vases décorés « d'arêtes de poisson ») avait déjà été remarquée au sein de fosses sur le site belge d'Irchonwelz « la Bonne Fortune », lui-même daté de la phase anciennemoyenne de la séquence du BVSG (Constantin et al. 1978). En l'état de nos connaissances, l'évolution des pratiques agricoles associées à un nouvel outillage en silex apparaîtrait donc plus anciennement dans la zone d'influence du groupe de Blicquy que dans le Bassin parisien.

\section{Des AFFINITÉS DIFFÉRENTES EN FONCTION DES SITES}

La position géographique des sites appelle à s'interroger sur leurs affinités avec les deux principaux bassins de peuplement : le Sud de la Belgique et le Bassin parisien.

Il y a de nombreux points communs entre les sites du groupe de Blicquy et ceux du Nord de la France comme le choix de s'implanter au sein d'un paysage mollement ondulé, en terrain lœssique (limons de plateau), à proximité de ressources aqueuses.

Les comparaisons avec le mobilier archéologique sont plus délicates mais plusieurs indices convergent pour émettre quelques hypothèses sur les filiations entre le Sud de la Belgique et le Bassin parisien. De toute évidence, les arguments sont nombreux concernant les affinités entre Loison-sous-Lens et les sites localisés dans le bassin de la Dendre : utilisation importante du dégraissant à l'os pilé dans la céramique, technique du décor en arêtes de poisson, panneaux poinçonnés couvrants, abondance de mobilier de mouture dans des contextes de dépôts / abandons organisés, introduction ancienne du tranchet, faiblesse des grattoirs dans l'outillage. Ces éléments suggèrent l'existence d'un particularisme régional de tradition plutôt blicquienne et de nombreux points communs notamment avec le gisement d'Irchonwelz (Constantin 1985).

Quant à Languevoisin-Quiquery, la proximité de la vallée de l'Oise, les comparaisons des corpus céramiques au niveau des formes et de l'utilisation d'un dégraissant sableux avec ceux d'Ocquerre «La Rocluche » (Seine-et-Marne ; Praud et al. 2009b), la forte représentation de silex bartonien dans l'outillage (la moitié), constituent des arguments établissant des liens privilégiés avec les gisements du nord du Bassin parisien.
Régionalement et comme on pouvait s'y attendre, les affinités se répartissent donc entre d'un côté les sites du bassin Deûle-Scarpe qui seraient liés à l'extrémité sud-occidental de l'expansion néolithique du groupe de Blicquy, et de l'autre ceux du bassin de la Somme plutôt sous influence du Bassin parisien.

\section{LOISON-SOUS-LENS : UN VILLAGE PIONNIER ?}

Les différentes études ont montré de multiples fois l'originalité de la composition du mobilier archéologique de l'habitat de Loison-sous-Lens, en particulier des spectres d'activités très tournés vers l'exploitation des matériaux végétaux. Localisé à environ $70 \mathrm{~km}$ des sites du Hainaut, on peut se demander dans quelle mesure nous ne sommes pas ici face à un site pionnier dans le mouvement de colonisation et de néolithisation d'espaces nouveaux, de la même manière que la question a été posée à l'occasion de l'étude du site de Colombelles pour le Rubané final du Bassin parisien (Billard et al. 2014). En effet, dans la perspective d'une nouvelle installation loin de ses bases, il est probable que les premières préoccupations des villageois se soient orientées vers la construction des maisons pour se protéger, mais aussi vers le développement des pratiques agricoles pour fournir les éléments de subsistance de base et développer l'agriculture de manière durable. Dans leur déplacement initial, les populations ont pu voyager, non seulement avec les animaux domestiques, ce que l'on ne peut pas ici appréhender faute de conservation de l'os, mais aussi avec des stocks de denrées et des effets personnels comme les vêtements. Ainsi, le travail des peaux ne présenterait pas un caractère d'urgence, ce qui pourrait expliquer le faible nombre de grattoirs dans la série. De même, la présence d'armatures de flèches en nombre pourrait évoquer une pratique de chasse renforcée pour permettre la multiplication des têtes de bétail et assurer la reproduction des espèces, hypothèse économique également formulée pour certaines maisons nouvellement implantées dans le Rubané final du bassin de la Seine à partir de l'analyse des maisons de Cuiry-lès-Chaudardes (Hachem et Hamon 2014, Gomart et al. 2015). Dans le même registre, la place importante des fruits sauvages (pomme, noisette, sureau) dans les restes carpologiques pourrait indiquer une contribution plus marquée des ressources sauvages dans l'alimentation. Dans le cadre d'une implantation nouvelle, on peut aussi envisager qu'une connaissance imparfaite de l'environnement et en particulier des ressources lithiques, ait pu amener les artisans à utiliser de manière opportuniste des supports différents (éclat à la place de lames) pour l'accomplissement de certaines activités très consommatrices de supports.

Ainsi, on pourrait déceler l'existence d'une hiérarchisation des priorités dans le cadre d'une installation nouvelle, celle de la subsistance des personnes étant sans doute la plus forte et générant une exploitation renforcée du milieu naturel végétal et animal. 


\section{QUelles IMPLICATIONS DANS LE PROCESSUS DE NÉOLITHISATION : UN RALENTISSEMENT DU FRONT DE COLONISATION?}

En l'état actuel de nos connaissances, l'espace géographique considéré correspond à une zone d'arrêt de l'expansion du Néolithique le plus ancien. Malgré le développement de l'archéologie préventive, aucun site rubané n'y a été mis au jour jusqu'à présent et seuls quelques sites pionniers datés du BVSG ont colonisé ces nouveaux espaces naturels lors de la phase d'extension maximale du Néolithique ancien dans la moitié nord de la France. Pour notre région, cela s'est fait à la fois depuis les sites du Hainaut belge et du Bassin parisien.

La nature des sols et le contexte géologique ne permettent pas d'expliquer le peu d'occupations danubiennes recensées sur notre secteur d'étude. La couverture limoneuse y est importante et se développe sur un substrat essentiellement crayeux formant un contexte environnemental particulièrement propice aux développements de pratiques agricoles et d'acquisition de matières premières lithiques. En effet, les ressources en silex crétacé et en grès tertiaire sont abondantes régionalement. L'absence de populations néolithiques avant 4950 cal. BC dans le Nord-Ouest de la France reste donc une question non résolue alors qu'en Hesbaye ou dans le Hainaut belge les sites les plus anciens datent de 5400/5300 av. J.-C. (Crombé et al. 2005). Ces derniers s'implantent dans des espaces naturels proches de ceux présents de l'autre côté de la frontière avec une accessibilité aux matières premières lithiques peut-être moins faciles du fait d'un substrat différent. Le contexte naturel ne peut donc pas être mobilisé pour expliquer cette absence de sites rubanés régionalement. $\mathrm{Ce}$ phénomène d'arrêt ou de stabilisation de la diffusion spatiale du Néolithique est observé à différentes échelles de temps et d'espaces depuis le Proche-Orient jusqu'aux confins de l'Europe occidentale (Rasse 2008). Un des facteurs responsables de ces situations et régulièrement mis en avant serait une densité importante de populations de chasseurs-cueilleurs bien installées sur ces territoires et hermétiques aux changements de mode de vie. Or, l'occupation du territoire pour notre secteur d'étude au cours de la phase finale du Mésolithique reste toute aussi discrète. Il faut préciser que ces sites sont difficiles à détecter dans ces contextes de fond de vallée et lorsqu'ils sont repérés, ils sont malheureusement rarement fouillés. En effet, parfois les séries mésolithiques sont mélangées à d'autres périodes plus anciennes ou plus récentes ou encore se trouvent en situation remaniée. Néanmoins, la récente découverte effectuée lors d'un diagnostic archéologique, d'une série lithique conséquente du Mésolithique final dans la vallée de la Marque au sud de Lille (Féray et al. 2016) a fait l'objet d'une date radiocarbone sur os calciné qui a donné comme résultat $6590 \pm 40$ BP (Beta439839), soit entre 5616 et 5481 cal. BC (à 95,4 \%). Mais là encore, l'absence de détermination de l'échantillon osseux et le ratio $\mathrm{C} 13 / \mathrm{C} 12$ à $28 \%$ invite à la plus grande prudence même si un facteur de correction a été appliqué. Dans l'attente de nouvelles mesures, l'intervalle de temps proposé par cette date est plus ancien que les premières manifestations rubanées régionales et extra-régionales. Pour cette phase terminale du Mésolithique, les sites de la vallée de la Somme, peu nombreux, ne permettent pas non plus d'avancer sur de possibles contacts ou relations entre ces populations (Ducrocq 2001). L'unique date concerne le site mésolithique du Castel $(6090 \pm 95$ BP, Gif-10419, soit 5290-4788 cal. BC) qui offre certes des intervalles de recouvrement avec le Néolithique ancien mais qui est trop imprécise (cinq siècles). Par ailleurs, la comparaison entre les industries lithiques du Mésolithique final et du Néolithique ancien (Allard 2007 et 2017), montre que si les armatures asymétriques peuvent être le témoin de contact entre les populations du Mésolithique final et celle du Néolithique ancien, les caractéristiques générales de l'industrie lithique du Rubané du Bassin parisien sont plus proches de celles de la tradition rubanée que de celles du Mésolithique.

Un autre facteur d'explication pourrait être lié à la physionomie du réseau hydrographique régional. En effet, les rivières qui traversent notre région sur un axe nord/sud ou bien est/ouest ont eu du mal à y creuser leur lit car les fonds de vallée ont été envahis par d'épais dépôts lœssiques du pléistocène supérieur. Elles ont un débit limité, une pente faible et s'inscrivent dans des vallées très larges favorisant le développement de vastes plaines humides pas toujours très praticables. A contrario, lorsque l'on observe la répartition des sites rubanés connus, on constate une distribution des gisements très localisée le long des grands axes fluviaux. Là où le réseau hydrographique est particulièrement peu propice à des déplacements sur de longues distances, des « isolats » d'occupations ont pu se former, à l'image des sites rubanés du Hainaut, qui ne trouvent pas immédiatement de voie à leurs expansions plus au sud, tandis que dans le Bassin parisien les vallées offrent des axes de pénétrations plus navigables sur un axe sud-est/nord-ouest permettant une conquête des sols jusqu'au rivage de la Manche (fig. 55).

La seconde phase du Néolithique ancien étend son emprise sur l'essentiel du territoire, dans toutes les directions conduisant jusqu'à la façade Atlantique/Manche mais notre secteur d'étude reste peu touché par ce mouvement d'expansion et l'extrémité nord-ouest du territoire est encore à l'écart d'une présence néolithique. Ainsi, dans l'hypothèse où ni les contraintes environnementales ni les contraintes humaines ne sont à l'origine de l'absence de sites du Néolithique ancien dans le quart nord-ouest de la France, il semble raisonnable de penser qu'on est ici face à une phase d'arrêt du processus d'expansion des cultures danubiennes, qui pour des raisons non encore expliquées aujourd'hui ne retrouveront pas de nouveau souffle mais, au contraire, disparaîtront à court terme.

Il faudra ainsi attendre le Cerny pour que quelques implantations, encore assez discrètes, s'intègrent au sein de ces nouveaux espaces sur la bande littorale de la Manche/mer du Nord, à un moment où partout ailleurs on enregistre un reflux du nombre d'occupations néolithiques sur la moitié nord de la France. Le Cerny régional se caractérise par quelques gisements qui pour les plus importants sont liés à l'exploitation de silex comme à Étaples « Les Sablins » (Piningre et al. 1991) ou à une occupation domestique livrant des bâtiments circulaires et un mobilier attribué à la phase récente de cette culture à Conty «ZAC Dunant» (Bostyn et al. 2016). Le 

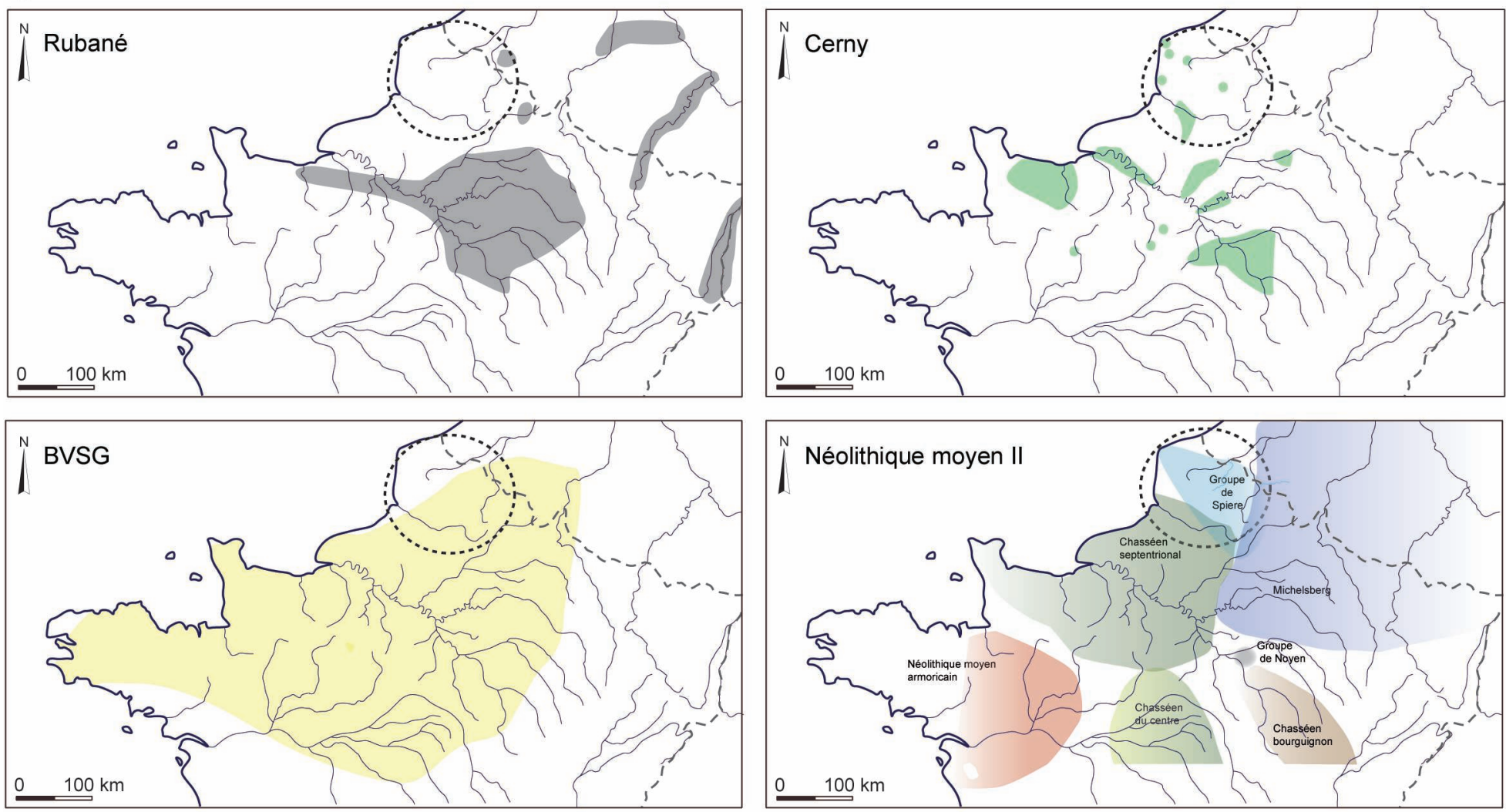

Fig. 55 - Densité des occupations du Néolithique ancien au Néolithique moyen 2 dans le Nord-Ouest de la France (I. Praud, Inrap).

Density of Early Neolithic to Middle Neolithic settlements in north-western France (I. Praud, Inrap).

reste se compose de fosses de rejets détritiques le plus souvent isolées mais qui confortent la présence néolithique sur le territoire régional. Il s'agit notamment d'une fosse dans le Boulonnais à Rinxent « rue J. Guesde » (Elleboode et Martial 2011) et dans la vallée de la Scarpe à Fresnes-les-Montauban « Chemin des Vaches » (Blancquaert et Desfossés 1992).

Ce n'est qu'à la seconde phase du Néolithique moyen que le modèle d'exploitation néolithique va s'étendre géographiquement (Monchablon et al. 2014) et conquérir tous les espaces avec une densité de gisements jamais atteinte jusqu'alors.

Ce schéma de la néolithisation à l'échelle régionale est finalement assez comparable avec ce qui a pu être observé dans la partie nord-occidentale de la plaine de l'Europe du Nord. Les interrogations et les problématiques sur l'extension néolithique sont assez similaires à celles rencontrées par nos collègues belges car seule la fin du $5^{\text {e }}$ et les débuts du $4^{\text {e }}$ millénaire correspondent à la colonisation intégrale de leur territoire intégrant les régions sableuses plus au nord qui jusque-là n'avaient pas livré de sites néolithiques (Crombé et al. 2005), soit quasiment plus d'un millénaire après les premières implantations sur les terrains limoneux méridionaux de Belgique. De la même manière, la néolithisation de la partie occidentale des Pays-Bas observée aux embouchures des deltas du Rhin et de la Meuse montre des changements irréversibles dans les modes de vie des populations de chasseurs-cueilleurs avec l'émergence des cultures du Néolithique moyen II (Louwe Kooijmans 2011) via des processus lents passant par des étapes d'appropriation de nouvelles stratégies techniques et économiques. Enfin, de l'autre côté du détroit de la Manche/Mer du Nord, en GrandeBretagne il faut attendre la transition des $5^{\mathrm{e}}$ et $4^{\mathrm{e}}$ millénaires pour voir se développer de manière durable les nouveaux modes de vie néolithiques (Whittle et al. 2011). 


\section{BIBLIOGRAPHIE}

Achard-Corompt N., Auxiette G., Fromont N., Ghesquiere E., Giazzon D., Kasprzyk M., Marcigny C., Riquier V. (2011) : Les fosses à profil « en V-Y-W » / Schllitzgruben : retour sur une énigme, in Bostyn F., Martial E., Praud I. (dir.), Le Néolithique du nord de la France dans son contexte européen. Habitat et économie aux $4^{e}$ et $3^{e}$ millénaires avant notre ère. Actes du $29^{e}$ colloque interrégional sur le Néolithique, Villeneuve d'Ascq, 2-3 octobre 2009, Senlis, Société archéologique de Picardie (nº spécial Revue archéologique de Picardie 28), p. 549-557.

Allard P. (1999) : L'industrie lithique du groupe de Villeneuve-SaintGermain des sites de Bucy-le-Long (Aisne), Revue archéologique de Picardie, 3-4, p. 53-114.

Allard P. (2005) : L'industrie lithique des populations rubanées $d u$ nord-est de la France et de la Belgique, Rahden, Verlag Marie Leidorf (Internationale Archäologie 86), 285 p.

Allard P. (2007) : The Mesolithic-Neolithic transition in the Paris Basin: a review, in Whittle A., Cummings V. (dir.), Going Over: The Mesolithic-Neolithic Transition in North-West Europe, Oxford, Oxford University Press (Proceedings of the British Academy 144), p. 213-224.

Allard P. (2017) : Variabilité des débitages laminaires au Second Mésolithique et au Néolithique ancien dans le nord de la France (VII ${ }^{e}$ et $\mathrm{VI}^{\mathrm{e}}$ millénaire BCE), Journal of Lithic Studies, 4 (2), p. $75-103$.

Allard P., Augereau A., Beugnier V., Burnez-Lanotte L., Bostyn F., Caspar J.-P., Giligny F., Hamard D., Martial E., Philibert S. (2004) : Fonction des outillages lithiques dans le Bassin parisien au Néolithique, in Bodu P., Constantin C. (dir.), Approches fonctionnelles en Préhistoire. Actes du XXVe congrès préhistorique de France, Nanterre, 24-26 novembre 2000, Paris, Société préhistorique française, p. 181-192.

Allard P., Bostyn F. (2006) : Genèse et évolution des industries lithiques danubiennes du Bassin parisien, in Allard P., Bostyn F., Zimmermann A. (dir.), Contribution des matériaux lithiques dans la chronologie du Néolithique ancien et moyen en France et dans les régions limitrophes. Actes de la Xe session de l'EAA, Lyon, septembre 2004, Oxford, Archaeopress (BAR Int. Ser. 1494), p. 28-55.

Ammerman A. J., Cavalli-Sforza L. (1971) : Measuring the rate of spread of Early Farming in Europe, Man, 6 (4), p. 674-688.

Augereau A. (2004) : L'industrie du silex $d u V^{e}$ au IV millénaire dans le sud-est du Bassin parisien. Rubané, Villeneuve-SaintGermain, Cerny et groupe de Noyen, Paris, Éditions de la MSH (Document d'archéologie française 97), 220 p.
Bailloud G. (1964) : Le Néolithique dans le Bassin parisien, Paris, CNRS Éditions (Suppl. Gallia Préhistoire 2), 433 p.

Bakels C. (2008) : L'agriculture rubanée/post-rubanée, continuité ou discontinuité ?, in Burnez-Lanotte L., Illet M., Allard P. (dir.), Fin des traditions danubiennes dans le Néolithique du Bassin parisien et de la Belgique (5100-4700 av. J.-C). Autour des recherches de Claude Constantin. Actes du colloque de Namur, 24-25 novembre 2006, Paris, Société préhistorique française (Mémoire 44) et Namur, Presses universitaires de Namur, p. 191-196.

Baudry A.-C., Cayol N., Bostyn F., Broes F., Buchez N., Coutard S., Derreumaux M., Dietsch-Sellami M.-F., Hulin G., Praud I., Vandamme N. (2013) : Du Néolithique ancien au premier âge du Fer : les indices d'occupations domestiques sur les communes de Breuil et Languevoisin-Quiquery (Somme), rapport final d'opération, Inrap, Canal Seine-Nord Europe, inédit, 243 p.

Bedaut L., Hachem L. (2008) : Recherches sur les sociétés du Néolithique danubien à partir du Bassin parisien : approche structurelle des données archéozoologiques, in Burnez-Lanotte L., Illet M., Allard P. (dir.), Fin des traditions danubiennes dans le Néolithique du Bassin parisien et de la Belgique (5100-4700 av. J.-C). Autour des recherches de Claude Constantin. Actes du colloque de Namur, 24-25 novembre 2006, Paris, Société préhistorique française (Mémoire 44) et Namur, Presses universitaires de Namur, p. 221-243.

Berger J.-F. (2009) : Climat, morphogénèse fluviale et néolithisation de l'Europe : quelques éléments de réflexion dans la perspective de diffusion arythmique de Jean Guilaine, in De Méditerranée et d'ailleurs... Mélanges offerts à Jean Guilaine, Toulouse, Archives d'écologie préhistorique, p. 63-82.

Beugnier V. (2000) : Étude fonctionnelle des microdenticulés, des racloirs à encoches et des tranchets du site d'Annoeullin (Nord), in Praud I. (dir.), Les occupations mésolithique et néolithique du site d'Annoeullin, rue Lavoisier, document final de synthèse, AFAN Nord-Picardie, inédit, $180 \mathrm{p}$.

Beugnier V., Crombe P. (2007) : L'outillage commun du premier site d'habitat découvert en Flandre (Belgique). Étude fonctionnelle de l'industrie lithique de Waardamme ( $3^{\text {e }}$ millénaire av. J.-C.), Bulletin de la Société préhistorique française, 104 (3), p. 525-542.

Blancquaert G., Desfossés Y. (1992) : L'occupation du Bronze final $\mathrm{du}$ "Chemin des Vaches » à Fresnes-lès-Montauban (Pas-deCalais), Bulletin de la Société préhistorique française, 8 (10-12), p. $429-438$.

Billard C., Bostyn F., Hamon C., Meunier K. (dir.) [2014] : L' habitat du Néolithique ancien de Colombelles « Le Lazzaro » (Calvados), Paris, Société préhistorique française (Mémoire 58), 408 p. 
Billard C., Ropars A., Pinel C., Marinval P., Habasque G., Bourhis J.-R., Blancquaert G., Aubry B. (1994) : Poses Le Vivier - Le Clos-Saint-Quentin (Eure). L'occupation de la plaine inondable au Néolithique et au début de l'âge du Bronze, Revue archéologique de l'Ouest, 11 (1), p. 53-113.

Bostyn F. (1994) : Caractérisation des productions et de la diffusion des industries lithiques du groupe néolithique du VilleneuveSaint-Germain, thèse de doctorat, Université Paris 10-Nanterre, inédit, 2 vol., 745 p.

Bostyn F. (1997) : Characterization of flint productions and distribution of the tabular Bartonian flint during the Early Neolithic (Villeneuve-Saint-Germain period) in France, in Schild R., Sulgostowska Z. (dir.), Man and Flint. Actes du VII International Flint Symposium, Varsovie, Septembre 1995, Varsovie, Institute of Archaeology and Ethnology of the Polish Academy of Sciences, p. 171-184.

Bostyn F. (2008) : Les importations en silex bartonien du Bassin parisien sur les sites blicquiens du Hainaut belge, in Burnez-Lanotte L., Illet M., Allard P. (dir.), Fin des traditions danubiennes dans le Néolithique $d u$ Bassin parisien et de la Belgique (5100-4700 av. J.-C). Autour des recherches de Claude Constantin. Actes du colloque de Namur, 24-25 novembre 2006, Paris, Société préhistorique française (Mémoire 44) et Namur, Presses universitaires de Namur, p. 397-411.

Bostyn F. (2009) : Quelques aspects de la néolithisation de la France, in Demoule J.-P. (dir.), La néolithisation dans le monde. Actes du colloque international de La Villette, octobre 2008, Paris, CNRS Éditions, p. 103-118.

Bostyn F., Arbogast R.-M., Cayol N., Hamon C., Lorin Y., Prodéo F. (2012) : Le site d'habitat Blicquy/Villeneuve-SaintGermain de Pontpoint « le Fond de Rambourg » (Oise), Gallia Préhistoire, 54, p. 67-189.

Bostyn F., Arbogast R.-M., Clavel B., Hamon C., Kuhar C., MaréchaL D., Pinard E., Praud I. (2015) : Habitat et sépultures du Blicquy/Villeneuve-Saint-Germain à Longueil-SainteMarie « le Barrage » (Oise), Revue archéologique de Picardie, 3-4, p. 155-206.

Bostyn F. (dir.), Ardouin S., Dietsch-Sellami M.-F., Hadjouis D., Marion S., Monchablon C., Pantano L., Praud. I., Prost D., Rimbault S., Rodriguez P., Verdin P. (2002) : Néolithique et protohistoire du site des Antes à Rungis (Val-de-Marne), Paris, Éditions ARPEA et Vitry-sur-Seine, Artcom, 183 p.

Bostyn F. (dir.), Beurion C., Billard C., Guillon M., Hachem L., Hamon C., Lanchon Y., Praud I., Reckinger F., Ropars A., Munaut A.-V. (2003a) : Néolithique ancien en HauteNormandie : le village Villeneuve-Saint-Germain de Poses "Sur la Mare » et les sites de la boucle du Vaudreuil, Société préhistorique française (Travaux 4), $342 \mathrm{p}$.
Bostyn F., Cayol N. (2012) : Productions de spécialistes, productions spécialisées : l'organisation des productions en silex sur les sites d'habitat du Villeneuve-Saint-Germain dans la moyenne vallée de l'Oise, Bulletin de la Société préhistorique française, 109 (2), p. 279-298.

Bostyn F., Charraud F., Denis S. (à paraître a) : Variabilités techniques, évolutions et aires d'influence des centres de productions laminaires au sein de la culture de Blicquy/Villeneuve-SaintGermain, in Bostyn F., Hamon C., Giligny F., Salavert A. (dir.), Actes du congrès préhistorique de France d'Amiens, 30 mai-4 juin 2016, Paris, Société préhistorique française.

Bostyn F., Denis S. (2016) : Specialised Production and Distribution Networks for Flint Raw Materials during the Blicquy-VilleneuveSaint-Germain Culture (Early Neolithic), in Kerig T., Nowak K., Roth G. (dir.), Alles was zählt... Festschrift für Andreas Zimmermann, Bonn, Habelt Verlag (Universitätsforschungen zur prähistorischen Archäologie 285), p. 195-208.

Bostyn F., Hachem L., Joseph F., Hamon C., Maigrot Y. (2016) : Apport du site d'habitat de Conty «ZAC Dunant » (Somme) à la connaissance de la culture de Cerny, Bulletin de la Société préhistorique française, 113 (2), p. 291-332.

Bostyn F., Ilett M., Meunier K. (à paraître b) : Tendances évolutives de l'organisation des habitats au Néolithique ancien (Rubané/B-VSG) dans le bassin de la Seine, in Habitations et habitat du Néolithique à l'âge du Bronze en France et ses marges. Actes des II rencontres Nord-Sud de Préhistoire récente, Dijon, 19-21 novembre 2015, Toulouse, Archives d'écologie préhistorique.

Bostyn F., Lanchon Y., Chambon P. (dir.) [2018] : Habitat $d u$ Néolithique ancien et nécropoles du Néolithique moyen I et II à Vignely, "la Porte aux Bergers ", Seine-et-Marne, Paris, Société préhistorique française (Mémoire 64), 454 p.

Bostyn F., Lemaire P., Prodéo F. (2003b) : Du Villeneuve-SaintGermain-Blicquy à mi-chemin entre Hainaut et Bassin parisien : le site de Vermand (Aisne), Bulletin de la Société préhistorique française, 100 (1), p. 165-172.

Bostyn F., Prodéo F., Valentin B., Blanchet J.-C. (1993) : L'occupation Villeneuve-Saint-Germain du site de LongueilSainte-Marie " La-butte-de-Rhuis » (Oise), in Le Néolithique au quotidien. Actes du XVI colloque interrégional sur le Néolithique, Paris, 5-6 novembre 1989, Paris, Éditions de la MSH (Document d'archéologie française 39), p. 26-40.

Boulen M., Deschodt L., Henton A. (2014) : Évolution morpho-sédimentaire et enregistrement pollinique atlantique dans le Nord de la France : la séquence de Valenciennes « Le Vignoble » (vallée de l'Escaut, Nord), Quaternaire, 25 (4), p. 369-389.

Brunet P., Lanchon Y. (2002) : Vignely «La Grande Pièce des Hayettes » (Seine-et-Marne), document final de synthèse et d'évaluation archéologique, SRA Île-de-France, Inrap, inédit, 15 p. 
Caspar J.-P. (1988) : Contribution à la tracéologie de l'industrie lithique du Néolithique ancien dans l'Europe nord-occidentale, thèse de doctorat, Université catholique de Louvain, inédit, 3 vol.

Caspar J.-P., Burnez-Lanotte L. (1996) : Groupe de BlicquyVilleneuve-Saint-Germain, nouveaux outils : le grattoirherminette et le foret, Bulletin de la Société préhistorique française, 93 (2), p. 235-240.

Caspar J.-P., Burnez-Lanotte L. (2008) : Les industries lithiques des cultures du Rubané et du Blicquy/Villeneuve-Saint-Germain : mise en convergences d'analyses croisées, in Burnez-Lanotte L., Illet M., Allard P. (dir.), Fin des traditions danubiennes dans le Néolithique du Bassin parisien et de la Belgique (51004700 av. J.-C). Autour des recherches de Claude Constantin. Actes du colloque de Namur, 24-25 novembre 2006, Paris, Société préhistorique française (Mémoire 44) et Namur, Presses universitaires de Namur, p. 245-268.

Caspar J.-P., Constantin C., Hauzeur A., Burnez-Lanotte L. (1993) : Nouveaux éléments dans le groupe de Blicquy en Belgique : le site de Vaux-et-Borset « Gibour » et « à la Croix Marie-Jeanne », Helinium, 32 (2), p. 168-252.

Caspar J.-P., Féray P., Martial E. (2005) : Identification et reconstitution des traces de teillage des fibres végétales au Néolithique, Bulletin de la Société préhistorique française, 102 (4), p. 867-880.

Caspar J.-P., Féray P., Martial E. (2007) : Le teillage des fibres végétales : pour une réinterprétation fonctionnelle d'outils en silex néolithiques, in Le Brun-Ricalens F., Valotteau F., Hauzeur A. (dir.), Relations interrégionales au Néolithique entre Bassin parisien et Bassin rhénan. Actes du $26^{e}$ colloque interrégional sur le Néolithique, Luxembourg, 8-9 novembre 2003, Luxembourg, Musée national d'Histoire et d'Art (Archaeologia Mosellana 7), p. 613-623.

Cayol N., Cravinho S., Dietsch-Sellami M.-F., Fechner K., Lanchon Y. (2015) : Une implantation du Néolithique ancien à Vitry-en-Artois-Chemin-Brûlé (Pas-de-Calais), rapport de fouille, Inrap Nord-Picardie, inédit, $180 \mathrm{p}$.

Charraud F. (2013) : Espaces interculturels et évolution des systèmes techniques au Néolithique dans le Nord-Ouest de la France. Productions, usages et circulation des outillages en silex jurassiques de Normandie, thèse de doctorat, Université Nice-Sophia Antipolis, inédit, 2 vol., 480 et 538 p.

Childe V. G. (1925) : The dawn of European Civilization, Londres, Kegan Paul, 539 p.

Clavel B. (2009) : Site de pêche néolithique au bord de l'Aisne, Archéopages, 26, p. 22-23.

Constantin C. (1985) : Fin du Rubané, céramique du Limbourg et post-rubané. Le Néolithique le plus ancien en Bassin parisien et en Hainaut, Oxford, BAR Publishing (BAR Int. Ser. 273), 2 vol.
Constantin C., Caspar J.-P., Hauzeur A., Burnez L., Sidéra I., Docquier J., Louboutin C., Tromme F. (1993) : Rubané et Groupe de Blicquy à Vaux-et-Borset (Gibour) (Hesbaye liégeoise), in Le Néolithique au quotidien. Actes du XVI colloque interrégional sur le Néolithique, Paris, 5-6 novembre 1989, Paris, Éditions de la MSH (Document d'archéologie française 39), p. 86-93.

Constantin C., Farruggia J.-P., Demarez L. (1991) : Le site rubané de Blicquy-La Couture du couvent (Hainaut). Fouilles 1983-8588, Bulletin des Chercheurs de la Wallonie, 31, p. 51-78.

Constantin C., Farrugia J.-P., Plateaux M., Demarez L. (1978) : Fouille d'un habitat néolithique à Irchonwelz (Hainaut occidental), Revue archéologique de l'Oise, 13, p. 3-19.

Constantin C., Ilett M. (1982) : Le Néolithique de Villeneuve-SaintGermain, in Vallée de l'Aisne : cinq années de fouilles protohistoriques, Senlis, Société archéologique de Picardie ( $\mathrm{n}^{\circ}$ spécial Revue archéologique de Picardie 1), p. 121-127.

Coudart A. (1998) : Architecture et société néolithique, Paris, Éditions de la MSH (Document d'archéologie française 67), 242 p.

Creusillet M.-F., Irribarria R. (2008) : Données récentes sur le Villeneuve-Saint-Germain du sud-ouest du Bassin parisien, in Burnez-Lanotte L., Illet M., Allard P. (dir.), Fin des traditions danubiennes dans le Néolithique du Bassin parisien et de la Belgique (5100-4700 av. J.-C). Autour des recherches de Claude Constantin. Actes du colloque de Namur, 24-25 novembre 2006, Paris, Société préhistorique française (Mémoire 44) et Namur, Presses universitaires de Namur, p.161-180.

Crombé P., Perdaen Y., Sergant J. (2005) : La néolithisation de la Belgique : quelques réflexions, in Marchand G., Tresset A. (dir.), Unité et diversité des processus de néolithisation sur la façade atlantique de l'Europe ( $6^{e}-4^{e}$ millénaires avant J.-C.). Actes de la table ronde de Nantes, 26-27 avril 2002, Paris, Société préhistorique française (Mémoire 36), p. 47-65.

Daniel R. (1973) : Le Campigny, commune de Blangy-sur-Bresles (Seine-Maritime). Outillage campignien provenant du fond de cabane classique, Bulletin de la Société préhistorique française, 70 (1), p. 13-16.

Denis S. (2008) : L'industrie lithique du site Villeneuve-SaintGermain de Vasseny (Aisne), mémoire de master 1, Université Paris 1 Panthéon Sorbonne, inédit, 2 vol.

Denis S. (2014a) : L'industrie lithique des populations blicquiennes (Néolithique ancien, Belgique) : organisation des productions et réseaux de diffusion, thèse de doctorat, Université Paris 10-Nanterre, inédit, 2 vol.

Denis S. (2014b) : The circulation of Ghlin flint during the time of the Blicquy - Villeneuve-Saint-Germain culture (Early Neolithic), Journal of Lithic Studies, 1 (1), p. 85-102. 
Dietsch-Sellami M.-F. (2007) : L'utilisation des plantes sauvages au Néolithique dans le nord-ouest de la France : témoignages carpologiques, in Besse M. (dir.), Sociétés néolithiques : des faits archéologiques aux fonctionnements socio-économiques. Actes du $27^{e}$ colloque interrégional sur le Néolithique, Neuchâtel, 1-2 octobre 2005, Lausanne, Musée cantonal d'Archéologie et Histoire (Cahiers d'archéologie romande 108), p. 115-122.

Dubouloz J. (2003) : Datation absolue du premier Néolithique du Bassin parisien : complément et relecture des données RRBP et VSG, Bulletin de la Société préhistorique française, 100 (4), p. 671-689.

Dubouloz J., Bocquet-Appel J.-P., Moussa R. (2017) : Modélisation, simulation et scénarios d'expérimentation. La colonisation LBK de l'Europe tempérée (5550-4950 av.n.e.), in Manolakakis L., Schlanger N., Coudart A. (dir.), Archéologie européenne, Identités et Migrations. Hommages à Jean-Paul Demoule, Leyde, Sidestone Press, p. 315-337.

Dubouloz J., Bostyn F., Chartier M., Cottiaux R., Le Bolloch M. (2005) : La recherche archéologique sur le Néolithique en Picardie, Revue archéologique de Picardie, 3-4, p. 63-98.

Ducrocq T. (2001) : Le Mésolithique du bassin de la Somme, insertion dans un cadre morpho-stratigraphique, environnemental et chronoculturel, Lille, Université des Sciences et technologies de Lille (Publications du CERP 7), 255 p.

Durand J. (dir.) [2004] : Mareuil-les-Meaux, Rocade sud-ouest de Meaux, lots 2 et 5, rapport de fouille, SRA Île-de-France, Inrap, inédit, $236 \mathrm{p}$.

Durand J. (dir.) [2007] : Rocade Ouest de Meaux (77) - Villenoy "Les Seize arpents », document final de synthèse, SRA Île-deFrance, Inrap, inédit, 157 p.

Elleboode E., Martial E. (2011) : Rinxent, rue Jules Guesde (Pasde-Calais), rapport de diagnostic, SRA Nord-Pas-de-Calais, Inrap, inédit, $30 \mathrm{p}$.

Fabre J. (dir.) [2007] : Géoarchéologie du silex du Nord-Ouest de la France, rapport de synthèse 2002-2006 du programme collectif de recherches, SRA Nord-Pas-de-Calais et Picardie, inédit, 204 p.

Farruggia J.-P., Constantin C., Demarez L. (1982) : Éléments non-rubanés du néolithique ancien entre les vallées du Rhin inférieur et de la Seine. V. Fouilles dans le groupe de Blicquy à Ormeignies, Irchonwelz, Aubechies (1977-1980), Hélinium, 22, p. 105-134.

Féray P., Auguste P., Créteur Y., Deschodt L., Lantoine J., Ledauphin A. (2016) : Château de Biscopp, station d'épuration intercommunale, Ennevelin (Nord), rapport de diagnostic, Inrap Hauts-de-France, inédit, 218 p.
Fromont N. (2003) : Anneaux en pierre et culture du VilleneuveSaint-Germain/Blicquy : premiers éléments sur la structuration des productions et la circulation des matières premières entre Massif armoricain et Massif ardennais, in Les matières premières lithiques en Préhistoire. Actes de la table ronde internationale d'Aurillac, 20-22 juin 2002, Cressensac, Association de préhistoire du Sud-Ouest (Préhistoire du Sud-Ouest 5), p. 177-184.

Fromont N. (2013) : Anneaux et cultures du Néolithique ancien. Production, circulation et utilisation entre massifs ardennais et armoricain, Oxford, Archaeopress (BAR Int. Ser. 2499), 686 p.

Fromont N., Constantin C., Vanguestaine M. (2008) : L'apport du site d'Irchonwelz à l'étude de la production des anneaux en schiste blicquiens (Néolithique ancien, Hainaut, Belgique), in Burnez-Lanotte L., Illet M., Allard P. (dir.), Fin des traditions danubiennes dans le Néolithique du Bassin parisien et de la Belgique (5100-4700 av. J.-C). Autour des recherches de Claude Constantin. Actes du colloque de Namur, 24-25 novembre 2006, Paris, Société préhistorique française (Mémoire 44) et Namur, Presses universitaires de Namur, p. 425-446.

Gaillard D., Gustiaux M., Bostyn F. (2003) : Loison-sous-Lens (62) "Parc d'activités les Oiseaux », rapport de diagnostic, SRA Nord-Pas-de-Calais, Inrap, inédit, 29 p.

Gassin B. (1996) : Évolution socio-économique dans le Chasséen de la grotte de l'Église supérieure (Var). Apport de l'analyse fonctionnelle des industries lithiques, Paris, CNRS Éditions (Monographie du CRA 17), 325 p.

Giligny F., (dir). Allard P., Augereau A., Beugnier V., Bostyn F., Burnez-Lanotte L., Caspar J.-P., Hamard D., Martial E., Philibert S. (2001): Fonction des outillages lithiques dans le Bassin parisien au Néolithique. Projet collectif de recherches, rapport final, SRA Île-de-France, inédit, $68 \mathrm{p}$.

Gomart L., Hachem L., Hamon C., Giligny F., Ilett M. (2015) : Household integration in Neolithic villages: A new model for the Linear Pottery Culture in west-central Europe, Journal of Anthropological Archaeology, 40, p. 230-249.

Gronenborn D. (1990) : Mesolithic-Neolithic Interactions - The lithic Industry of the Earliest Bandkeramik Culture Site at Friedberg-Bruchenbrücken, Wetteraukreis (West Germany), in Vermeersch P., Van Peer P. (dir.), Contributions to the Mesolithic in Europe, Louvain, Leuven University Press, p. 173-182.

Guilaine J. (2001) : La diffusion de l'agriculture en Europe : une hypothèse arythmique, Zephirus, 53-54, p. 267-272.

Hachem L., Hamon C. (2014) : Linear pottery household organization: an economic model, in Whittle A., Bickle P. (dir.), Early Farmers: The View from Archaeology and Science, Oxford, Oxford University Press (Proceedings of the British Academy 198), p. 159-180. 
Hamon C. (2003) : Les outils de mouture, percussion et polissage du site de Poses «Sur la Mare», in Bostyn F. (dir.), Néolithique ancien en Haute-Normandie : le village Villeneuve-Saint-Germain de Poses "Sur la Mare " et les sites de la boucle du Vaudreuil, Société préhistorique française (Travaux 4), p. 267-279.

Hamon C. (2006) : Broyage et abrasion au Néolithique ancien. Caractérisation technique et fonctionnelle des outillages en grès du Bassin parisien, Oxford, Archaeopress (BAR Int. Ser. 1551), $342 \mathrm{p}$.

Hamon C. (2008) : Meules rubanées, meules blicquiennes : nouvelles réflexions sur les dépôts du Hainaut (Belgique), in BurnezLanotte L., Illet M., Allard P. (dir.), Fin des traditions danubiennes dans le Néolithique du Bassin parisien et de la Belgique (5100-4700 av. J.-C). Autour des recherches de Claude Constantin. Actes du colloque de Namur, 24-25 novembre 2006, Paris, Société préhistorique française (Mémoire 44) et Namur, Presses universitaires de Namur, p. 197-208.

Hamon C. (2009) : L'outillage en grès, in Praud I. (dir.), Le Néolithique ancien dans la Basse vallée de la Marne et ses affluents : un site d'habitat producteur de lames en silex tertiaire à Ocquerre "La Rocluche » (Seine-et-Marne), Paris, Société préhistorique française (Travaux 9), p. 77-97.

Hamon C. (2013) : Le macro-outillage lithique, in Lanchon Y., Bostyn F. (dir.), Un hameau du Néolithique ancien. Le Pré aux Bateaux à Luzancy (Seine-et-Marne), Paris, CNRS Éditions et Inrap (Recherches archéologiques 6), p. 115-133.

Hamon C., Samzun A. (2004) : Une fosse Villeneuve-SaintGermain final à Saint Denis « Rue du Landy » : un dépôt de meule inédit en Île-de-France, in Internéo 5. Actes de la journée d'information de Paris, 20 novembre 2004, Paris, Société préhistorique française, p. 17-28.

Hauzeur A. (2008) : Céramique et périodisation : essai de sériation du corpus blicquien de la culture de Blicquy/Villeneuve-SaintGermain, in Burnez-Lanotte L., Illet M., Allard P. (dir.), Fin des traditions danubiennes dans le Néolithique du Bassin parisien et de la Belgique (5100-4700 av. J.-C). Autour des recherches de Claude Constantin. Actes du colloque de Namur, 24-25 novembre 2006, Paris, Société préhistorique française (Mémoire 44) et Namur, Presses universitaires de Namur, p. 129-142.

Hébert S. (2009) : Boves «Les Longues Haies » (Somme), rapport de diagnostic, Inrap Picardie, inédit, $27 \mathrm{p}$.

Henton A., Boulen M., Deschodt L. (2009) : Valenciennes, le Vignobles $I V$, rapport final d'opération, SRA Nord-Pas-deCalais, Inrap, inédit, 57 p.

Hosdez C. (2001) : Noyelles-sous-Lens (62) " Parcelle Bertelsman Services », rapport de fouille, SRA Nord-Pas-de-Calais, Inrap, inédit, $40 \mathrm{p}$.
Jadin I. (2003) : Trois petits tours et puis s'en vont... La fin de la présence danubienne en Moyenne Belgique, Liège, Presses universitaires de Liège (ERAUL 109), $721 \mathrm{p}$.

Joseph F., Julien M., Leroy-Langelin E., Lorin Y., Praud I. (2011) : L'architecture domestique des sites du III $^{e}$ millénaire avant notre ère dans le Nord de la France, in Bostyn F., Martial E., Praud I. (dir.), Le Néolithique du nord de la France dans son contexte européen. Habitat et économie aux $4^{e}$ et $3^{e}$ millénaires avant notre ère. Actes du $29^{e}$ colloque interrégional sur le Néolithique, Villeneuve d'Ascq, 2-3 octobre 2009, Senlis, Société archéologique de Picardie ( ${ }^{\circ}$ spécial Revue archéologique de Picardie 28), p. 249-273.

Lanchon Y. (1984) : Le Néolithique danubien et de tradition danubienne dans l'Est du Bassin parisien, mémoire de maîtrise, Université Paris 1 Panthéon-Sorbonne, inédit, 2 vol.

Lanchon Y. (2008) : La culture de Blicquy/Villeneuve-SaintGermain dans la basse vallée de la Marne : première approche chronologique à partir de la céramique, in Burnez-Lanotte L., Illet M., Allard P. (dir.), Fin des traditions danubiennes dans le Néolithique du Bassin parisien et de la Belgique (5100-4700 av. J.-C). Autour des recherches de Claude Constantin. Actes du colloque de Namur, 24-25 novembre 2006, Paris, Société préhistorique française (Mémoire 44) et Namur, Presses universitaires de Namur, p. 143-159.

Lanchon Y. (2012) : Le Néolithique ancien dans la basse vallée de la Marne. Premières réflexions sur les sites, leur statut et leur organisation territoriale, in Carpentier V., Marcigny C. (dir.), Des hommes aux champs. Pour une archéologie des espaces ruraux du Néolithique au Moyen Âge, Rennes, Presses universitaires de Rennes (Archéologie et Culture), p. 35-54.

Lanchon Y., Allenet de Ribemont G., André M.-F., Bedault L., Boitard È., Bonnardin S., Bostyn F., Brunet P., Cayol N., Cottiaux R., Durand J., Fromont N., Hachem L., Hamon C., Lejeune Y., Leroyer C., Martial E., Maigrot Y., Meunier K., Praud I., Thévenet $\mathbf{C}$. (2006) : Action collective de recherche : "Le Néolithique ancien dans la Basse Vallée de Marne ", rapport d'activité ( $2^{\mathrm{e}}$ année), inédit, 2 vol.

Lanchon Y., Allenet de Ribemont G., André M.-F., Bonnardin S., Bostyn F., Boulanger L., Brunet P., Cottiaux R., Durand J., Hachem L., Hamon C., Le Jeune Y., Leroyer C., Martial E., Maigrot Y., Meunier K., Pastre J.-F., Praud I., Thévenet C. (2005) : Action collective de recherche: "Le Néolithique ancien dans la Basse Vallée de Marne ", rapport d'activité ( $1^{\mathrm{re}}$ année), SRA Île-de-France, inédit, 2 vol.

Lefranc P. (2014) : Les villages du Néolithique ancien en Alsace. Un état de la recherche, Archéopages, 40, p. 18-25.

Louboutin C., Simonin D. (1997) : Le Cerny-Videlles : un faciès ancien de la culture de Cerny, in Constantin C., Mordant D., Simonin D. (dir.), La culture de Cerny, nouvelle économie, 
nouvelle société au Néolithique. Actes du colloque de Nemours, 9-11 mai 1994, Nemours, APRAIF (Mémoire du musée de Préhistoire d'Île-de-France 6), p. 135-167.

Louwe Kooijmans L.-P. (2011) : Schipluiden and the Final Stage of Neolithisation in the Lower Rhine Basin, in Bostyn F., Martial E., Praud I. (dir.), Le Néolithique du nord de la France dans son contexte européen. Habitat et économie aux $4^{e}$ et $3^{e}$ millénaires avant notre ère. Actes du $29^{e}$ colloque interrégional sur le Néolithique, Villeneuve d'Ascq, 2-3 octobre 2009, Senlis, Société archéologique de Picardie ( $\mathrm{n}^{\circ}$ spécial Revue archéologique de Picardie 28), p. 121-138.

Meunier K. (2012) : Styles céramiques et néolithisation dans le sud-est $d u$ Bassin parisien, Paris, CNRS Éditions et Inrap (Recherches archéologiques 5), 273 p.

Meunier K., Bostyn F., Cayol N., Hamon C. (2014) : De la fosse à la culture : acquis et perspective de recherches sur le Néolithique ancien dans le Bassin parisien, in Sénépart I., Billard C., Bostyn F., Praud I., Thirault É. (dir.), Méthodologie des recherches de terrain de la Préhistoire récente en France. Nouveaux acquis, nouveaux outils 1987-2012. Actes des premières rencontres Nord/Sud de Préhistoire récente, 23-25 mai 2012, Toulouse, Archives d'écologie préhistorique, p. 273-286.

Monchablon C. (2005) : Le matériel de mouture, de broyage et de polissage, in Giligny F. (dir.), Louviers "La Villette » (Eure). Un site néolithique moyen en zone humide, Rennes, Presses universitaires de Rennes (Document archéologique de l'Ouest), p. 149-161.

Monchablon C. (2015) : Étude de l'assemblage en grès, in Praud I. (dir.) Le Néolithique final dans la vallée de la Deûle. Le site d'Houplin-Ancoisne, le Marais de Santes, Paris, CNRS Éditions et Inrap (Recherches archéologiques 9), p. 229-255.

Monchablon C., Bostyn F., Praud I. (2014) : Questions de méthodes et problématiques dans les fouilles des enceintes du Néolithique moyen II : exemples dans le Nord de la France, in Sénépart I., Billard C., Bostyn F., Praud I., Thirault É. (dir.), Méthodologie des recherches de terrain de la Préhistoire récente en France. Nouveaux acquis, nouveaux outils 19872012. Actes des premières rencontres Nord/Sud de Préhistoire récente, 23-25 mai 2012, Toulouse, Archives d'écologie préhistorique, p. 203-215.

Mordant C., Mordant D., collab. Bontillot J., Paris J. (1977) : Le Bois des Refuges à Misy-sur-Yonne (Seine-et-Marne), Bulletin de la Société préhistorique française. Études et travaux, 74 (1), p. $420-462$.

Oberlin C. (2003) : Calibration des datations radiocarbone : le point sur la période $6^{\mathrm{e}}-2^{\mathrm{e}}$ millénaire avant J.-C., in Gascó J., Gutherz X., Labriffe P.-A. de (dir.) Temps et espaces culturels du $6^{e}$ au $2^{e}$ millénaire en France du Sud. Actes des 4 e rencontres méridionales de
Préhistoire récente, Nîmes, 28-29 octobre 2000, Lattes, Association pour le développement de l'archéologie en Languedoc-Roussillon (Monographies d'archéologie méditerranéenne 15), p. 35-42.

Pavúk J. (2004) : Early Linear Pottery culture in Slovakia and the neolithisation of central Europe, in Lukes A., Zvelebil M. (dir.) LBK Dialogues. Studies in the Formation of the Linear Pottery Culture, Oxford, Archaeopress (BAR Int. Ser. 1304), p. 71-82.

Philibert S. (1996) : Approche techno-fonctionnelle des outillages du Néolithique ancien et moyen dans le Bassin parisien. Les gisements du Moulin de Lettrée (Neauphle-le-Vieux) et de Louviers (Eure) : premiers résultats, in Internéo 1. Actes de la journée d'information de Paris, 23 novembre 1996, Paris, Société préhistorique française, p. 33-44.

Philibert S. (2005) : Analyse fonctionnelle de l'outillage en silex, in Giligny F. (dir.), Louviers « La Villette ». Un site néolithique moyen en zone humide, Rennes, Presses universitaires de Rennes (Document archéologique de l'Ouest), p. 133-148.

Piningre J.-F., Bostyn F., Couppé J., collab. Constantin C., Delibrias G. (1991) : L'atelier de taille du silex des Sablins à Étaples (Pas-de-Calais), Gallia Préhistoire, 33, p. 83-135.

Praud I., Bostyn F., Cayol N., Dietsch-Sellami M.-F., Clavel V., Hamon C., Pinard E, Ladureau P., Lanchon Y. (2009a) : $U n$ site d'habitat du Néolithique ancien à Loison-sous-Lens (Pasde-Calais), rapport final de fouille, Inrap Picardie, inédit, $125 \mathrm{p}$.

Praud I., Bostyn F., Cayol N., Hamon C., Ladureau P., Lanchon Y., Pinard E. (2010) : Entre Blicquy et Villeneuve-Saint-Germain. Présentation de la fouille d'un habitat du Néolithique ancien à Loison-sous-Lens (Pas-de-Calais), in Billard C., Legris M. (dir.), Premiers néolithiques de l'Ouest. Cultures, réseaux, échanges des premières sociétés néolithiques à leur expansion. Actes du $28^{e}$ colloque interrégional sur le Néolithique, Le Havre, 9-10 novembre 2007, Rennes, Presses universitaires de Rennes (Archéologie et Culture), p. 305-323.

Praud I., Bostyn F., Creteur Y., Henton A., Hosdez C., Martial E., Poirier C. (2005) : Loison-sous-Lens (62) Parc d'activités «Les Oiseaux ", rapport de diagnostic, Inrap Picardie, SRA NordPas-de-Calais, inédit, $51 \mathrm{p}$.

Praud I. (dir.), Bostyn F., Hamon C., Lanchon Y., collab. Caspar J.-P., Dietsch-Sellami M.-F., Martial E., Michel L., Vachard D. (2009b) : Le Néolithique ancien dans la basse vallée de la marne : un site Villeneuve-Saint-Germain producteur de lames en silex tertiaire à Ocquerre "La Rocluche » (Seine-et-Marne), Paris, Société préhistorique française (Travaux 9), $137 \mathrm{p}$.

Praud I., collab. Le Gall J., Vachard D. (2003) : Les bracelets en pierre du Néolithique ancien : provenance et diffusion des matériaux sur les sites Villeneuve-Saint-Germain du Bassin parisien, in Desbrosse R., Thévenin A. (dir.), Préhistoire de 
l'Europe. Des origines à l'âge du Bronze. Actes du colloque $d u$ CTHS, Lille, 10-15 avril 2000, Paris, Éditions du CTHS, p. 491-502.

Prestreau M. (1992) : Le site néolithique et protohistorique des Falaises de Prépoux à Villeneuve-la-Guyard (Yonne), Gallia Préhistoire, 34, p. 171-207.

Prodéo F., Defaux F., Deschodt L., Harnay V., Lancelot S., Montaru D., Swinnen C., Talon M. (1997) : Étouvie "Le Chemin de la Marine ", document final de synthèse, AFAN, SRA Picardie, inédit, $251 \mathrm{p}$.

Rasse M. (2008) : La diffusion du Néolithique en Europe (7000-5000 av. J.-C.) et sa représentation cartographique, M@ppemonde, 90 (2) [URL : https://mappemonde-archive.mgm.fr/num18/ articles/art08205.html]

Rasse M. (2014) : Modélisation de la diffusion du Néolithique en Europe, M@ppemonde, 115 (3) [URL : http://mappemonde. mgm.fr/num43/articles/art14302.html].

Reniere S., Dreesen R., Fronteau G., Gluhak T., Goemaere E., Hartoch E., Picavet P., De Clereq W. (2016) : Querns and mills during Roman times at the northern frontier of the Roman Empire (Belgium, northern France, southern Netherlands, western Germany): Unravelling geological and geographical provenances, a multidisciplinary research project, Journal of Lithic Studies, 3 (3), p. 403-428.

Simonin D. (1997) : La transition Villeneuve-Saint-Germain/Cerny dans le Gâtinais et le Nord-est de la Beauce, in Constantin C., Mordant D., Simonin D. (dir.), La culture de Cerny, nouvelle économie, nouvelle société au Néolithique. Actes du colloque de Nemours, 9-11 mai 1994, Nemours, APRAIF (Mémoire du musée de Préhistoire d'Île-de-France 6), p. 39-64.

Stadler P., Kotova N. (2013) : The longhouses from Brunn Wolfholz, distribution of finds and the importance of the different sites for the development of the Linear Pottery culture, in Hamon C., Allard P., Ilett M. (dir.), The Domestic Space in LBK Settlements, Rahden, Verlag Marie Leidorf (Internationale Archäologie 17), p. 51-77.
Thévenet C. (2010) : Des faits aux gestes...des gestes aux sens? Pratiques funéraires et société durant le Néolithique ancien en Bassin parisien, thèse de doctorat, Université Paris 1 PanthéonSorbonne, inédit, 811 p.

Vacossin J.-F., Cayol N., Joseph F. (2010) : Sancourt " Grande Rue / Rue du Moulin » (Somme), Rapport de diagnostic, Inrap Picardie, inédit, $34 \mathrm{p}$.

Van Gijn A.-L. (2010) : Flint in Focus: Lithic Biographies in the Neolithic and Bronze Age, Leyde, Sidestone Press, 289 p.

Vaughan P. C. (1994) : Microwear analysis on flints fron the bandkeramik sites of Langweiler 8 and Laurenzberg 7, in Lüning J., Stehli P. (dir.), Die Bandkeramik im Merzbachtal auf der Aldenhovener platte, Cologne, Rheinland Verlag (Rheinisch Ausgrabungen 36), p. 533-558.

Whittle A. (1990) : Radiocarbon dating of the Linear Pottery Culture: the contribution of cereal and bone samples, Antiquity, 64 (243), p. 297-302.

Whittle A. (2007) : Going over: people and their times, in Whittle A., Cummings V. (dir.), Going Over: The Mesolithic-Neolithic Transition in North-West Europe, Oxford, Oxford University Press (Proceedings of the British Academy 144), p. 617-628.

Whittle A., Healy F., Bayliss A. (2011) : Rassembler le temps : la datation des enceintes à fossés interrompus du Néolithique ancien du sud de la Grande-Bretagne, in Bostyn F., Martial E., Praud I. (dir.), Le Néolithique du nord de la France dans son contexte européen. Habitat et économie aux $4^{e}$ et $3^{e}$ millénaires avant notre ère. Actes du $29^{e}$ colloque interrégional sur le Néolithique, Villeneuve d'Ascq, 2-3 octobre 2009), Senlis, Société archéologique de Picardie ( ${ }^{\circ}$ spécial Revue archéologique de Picardie 28), p. 41-54.

Zazzo A, Saliège J-F., Person A, Boucher H. (2009) : Radiocarbon dating of calcined bones: where does the carbon come from?, Radiocarbon, 51 (2), p. 601-611. 